\title{
Detailed Investigations into the Propagation and Termination Kinetics of Bulk Homo- and Copolymerization of (Meth)Acrylates
}

\author{
Dissertation \\ zur Erlangung des Doktorgrades \\ der Mathematisch-Naturwissenschaftlichen Fakultäten \\ der Georg-August-Universität zu Göttingen
}

vorgelegt von

Elena Müller (geb. Panchenko)

aus Jarowoje / Russland

Göttingen 2005 
D 7

Referent:

Prof. Dr. M. Buback

Korreferent:

Prof. Dr. K. Hoyermann

Tag der mündlichen Prüfung:

28.04.2005 


\section{TABLE OF CONTENTS}

1. ABSTRACT

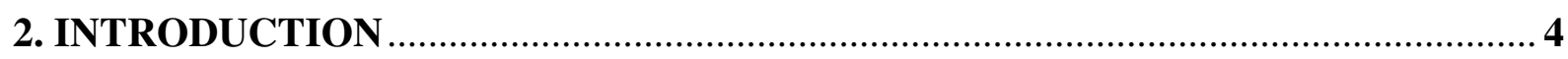

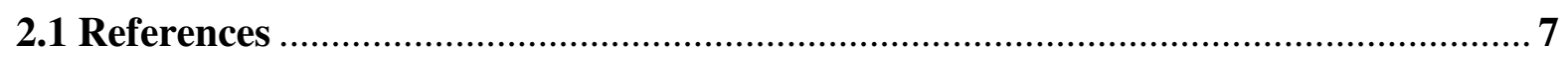

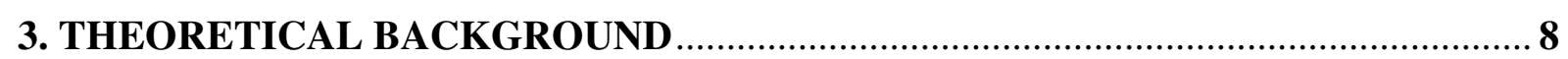

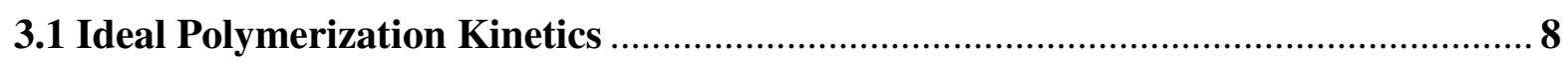

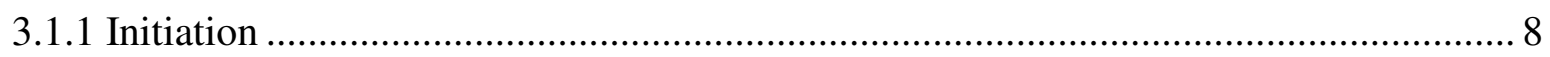

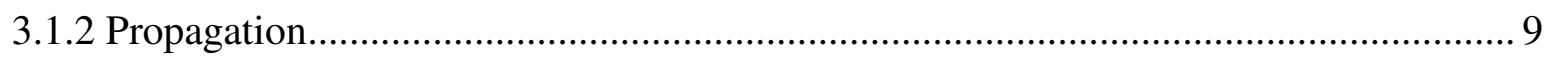

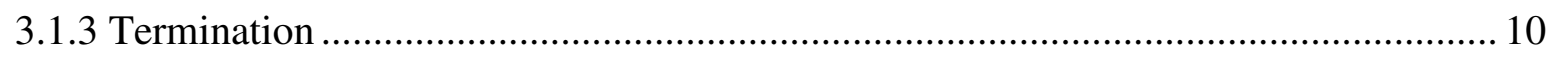

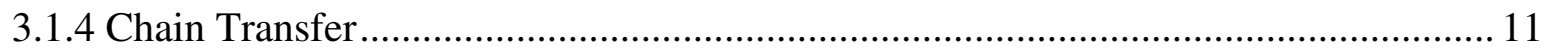

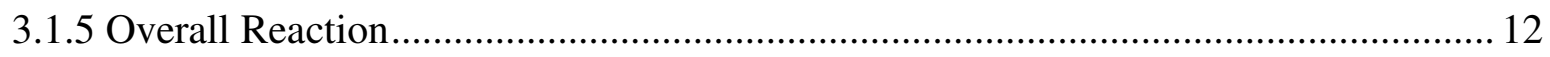

3.2 Determination of Propagation and Termination Rate Coefficients ....................... 12

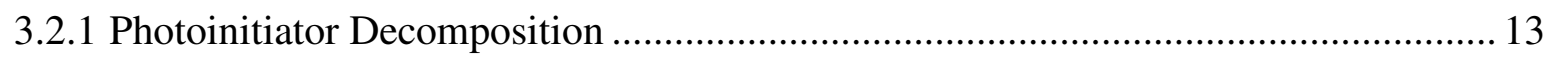

3.2.2 Determination of the Propagation Rate Coefficient, $k_{\mathrm{p}}$, with

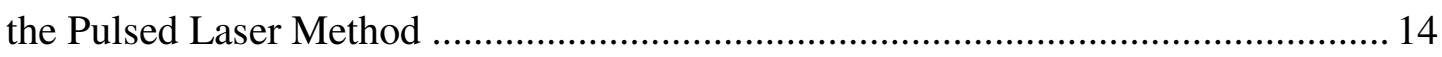

3.2.2.1 Determination of the Molecular Weight Distribution ......................................... 15

3.2.3 Determination of the Coupled Parameters $k_{\mathrm{p}} \cdot k_{\mathrm{t}}^{-0.5}$ from Chemically Initiated

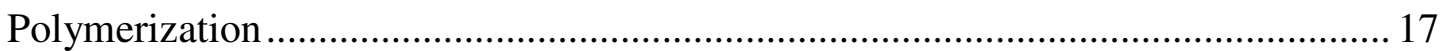

3.2.4 Determination of the Termination Rate Coefficient, $k_{\mathrm{t}}$, from the SP-PLP-NIR

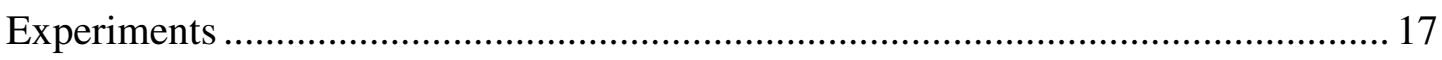

3.2.5 Determination of the Termination Rate Coefficient, $k_{\mathrm{t}}$, from the SP-PLP-ESR

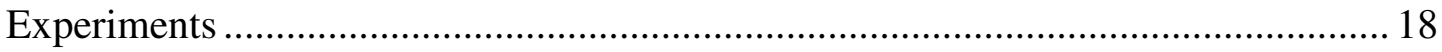

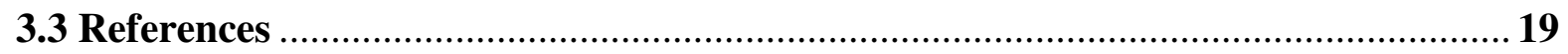

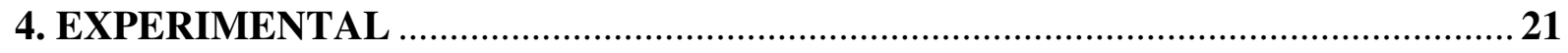

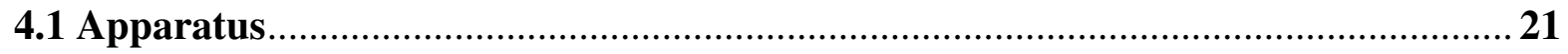

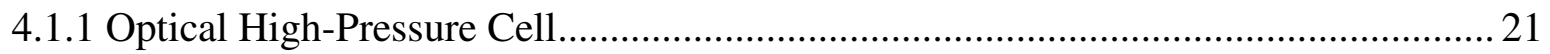

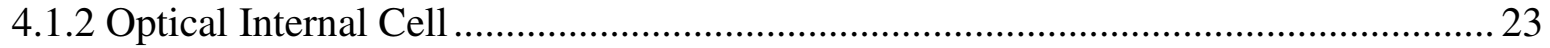

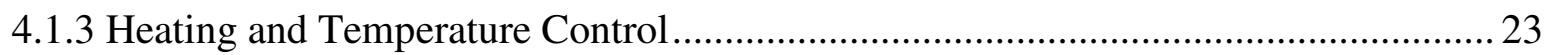




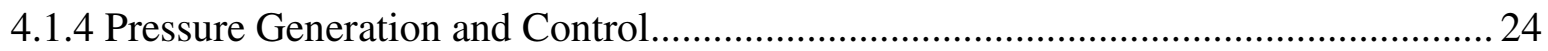

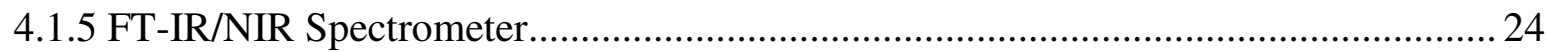

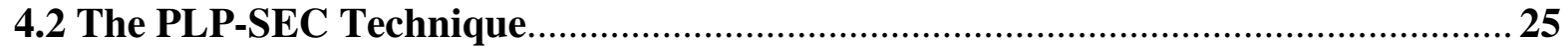

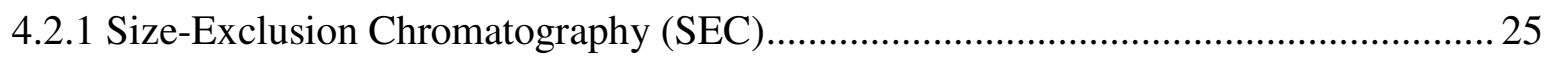

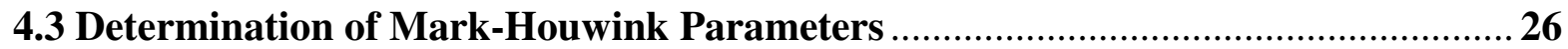

$4.4{ }^{1}$ H-NMR

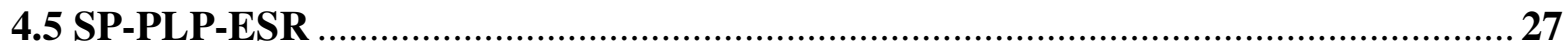

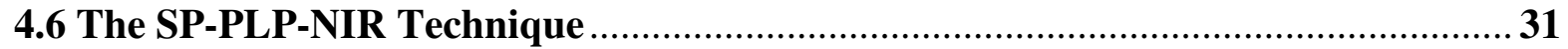

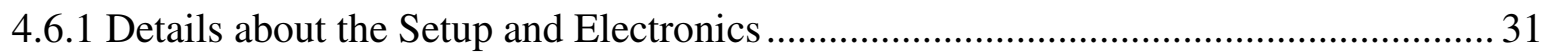

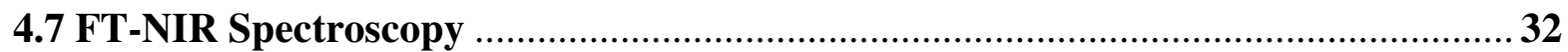

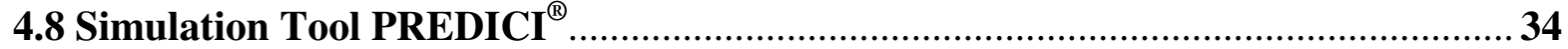

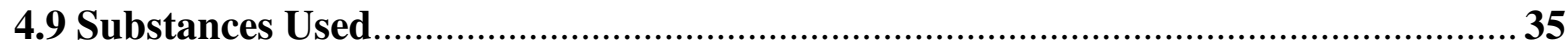

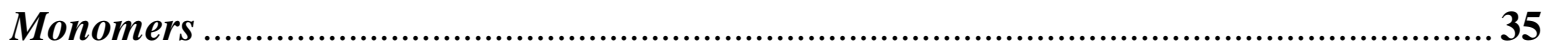

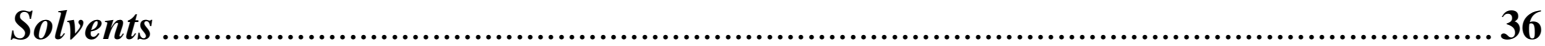

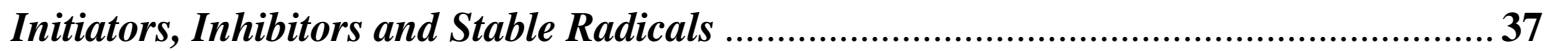

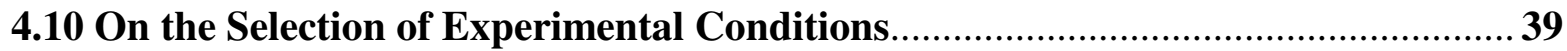

4.10.1 Laser-Induced Polymerization in PLP-SEC Experiments ........................................ 39

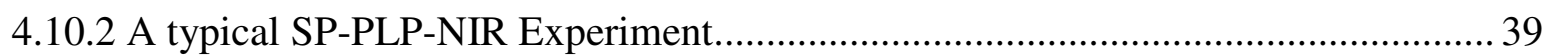

4.10.3 Laser-Induced Polymerization in SP-PLP-ESR ....................................................... 40

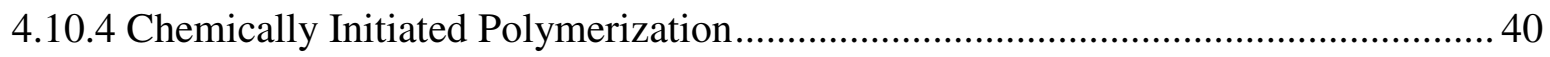

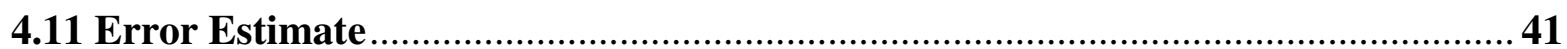

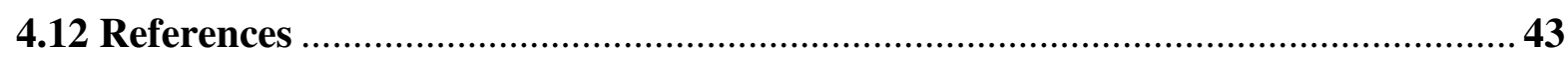

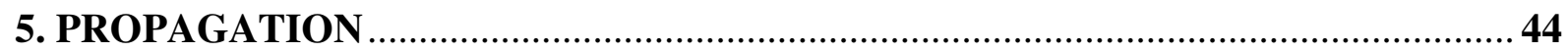

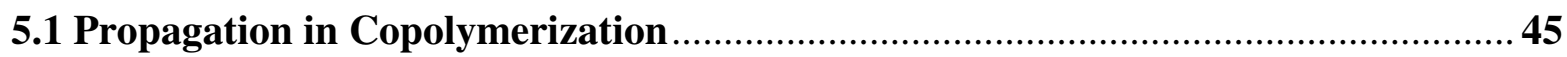

5.1.1 Kinetic Models for Free-Radical Copolymerization ............................................... 45 


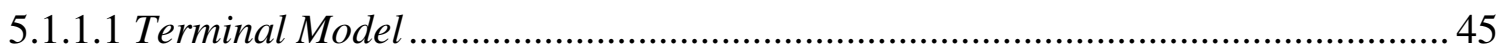

5.1.1.2 Explicit and Implicit Penultimate Unit Models ................................................... 46

5.1.2 Determination of Copolymer Composition by ${ }^{1} \mathrm{H}-\mathrm{NMR}$ Spectroscopy...................... 49

5.1.3 Experimental Results and Data Analysis by PUE Models........................................ 53

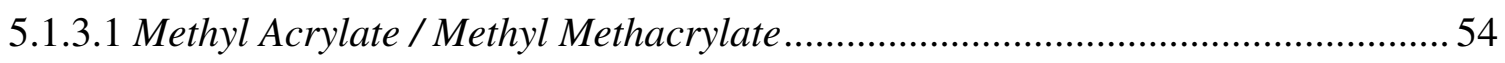

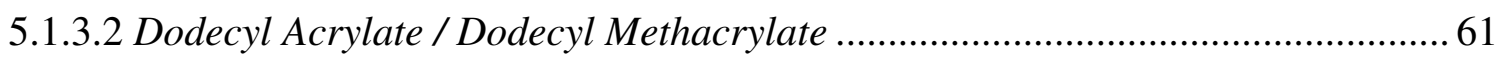

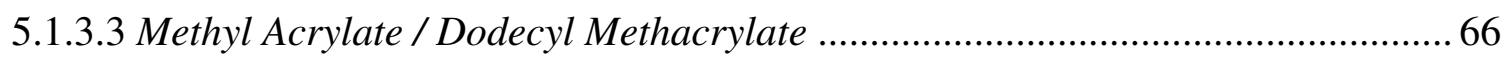

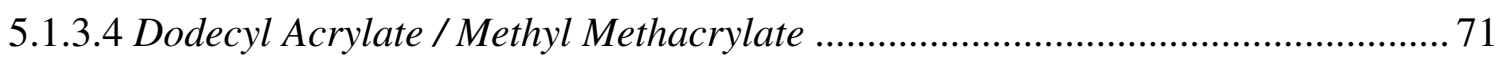

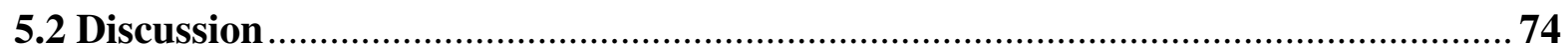

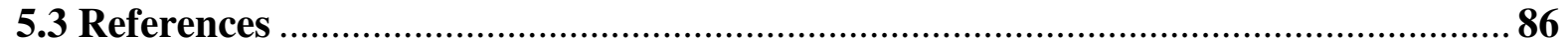

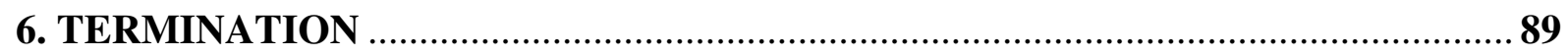

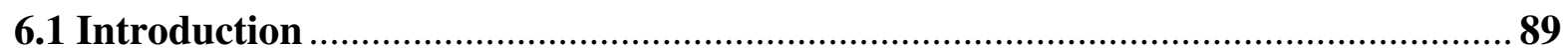

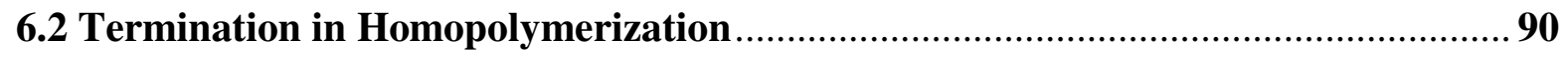

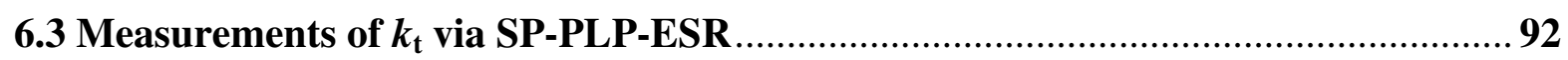

6.3.1 Dependence of $k_{\mathrm{t}}(\mathrm{DMA})$ on Monomer Conversion ................................................ 93

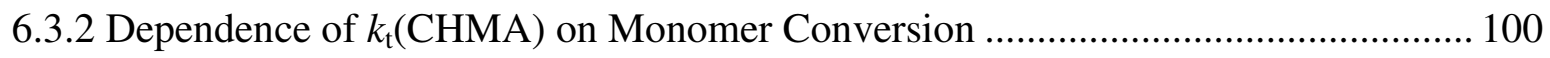

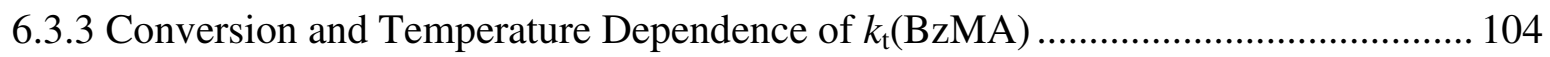

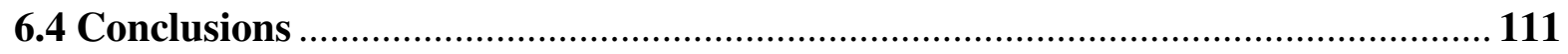

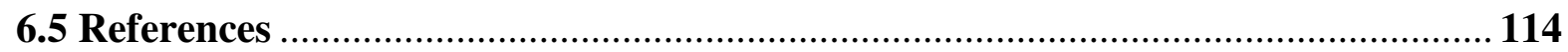

7. STUDY INTO THE OCCURRENCE OF SECONDARY PROPAGATING

AND MID-CHAIN RADICALS VIA ESR IN FREE-RADICAL ACRYLATE

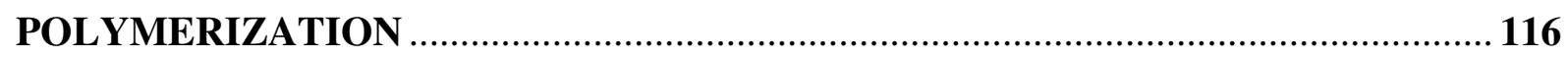

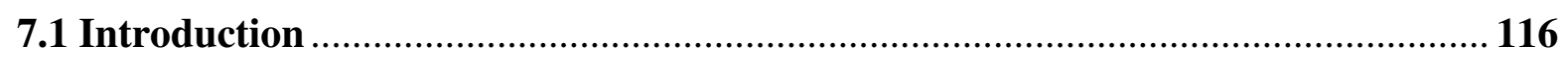

7.2 ESR measurements of Propagating Radicals of Acrylates..................................... 119

7.2.1 ESR Measurements during BA Polymerization.................................................. 119

7.2.2 ESR Measurements during DA Polymerization................................................... 129

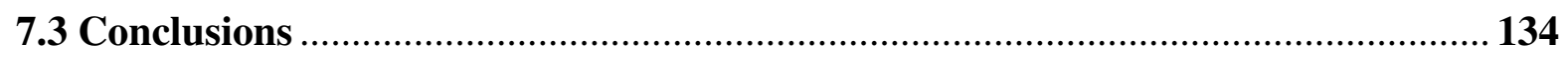




\section{DETERMINATION OF PROPAGATION RATE COEFFICIENTS, $k_{\mathrm{p}}$, FOR} THE DODECYL ACRYLATE DIMER (DAD).

8.1 Introduction.

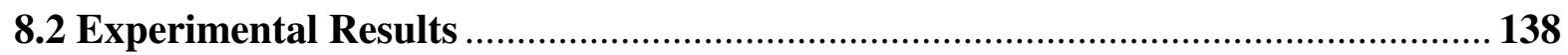

8.2.1 Estimation of Mark-Houwink Parameters............................................................... 139

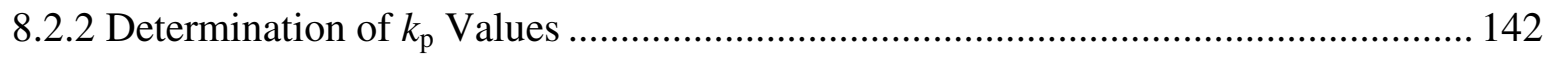

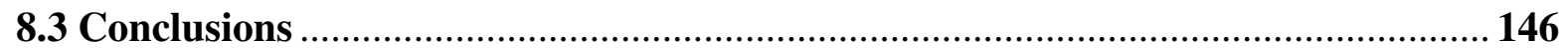

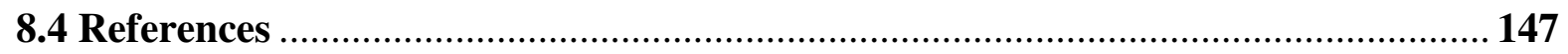

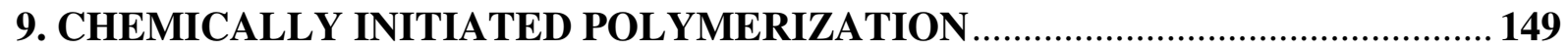

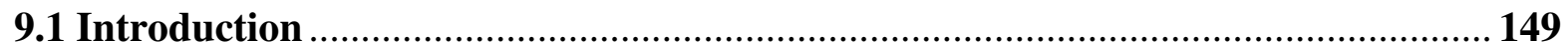

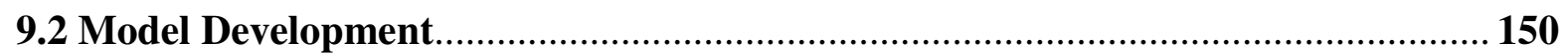

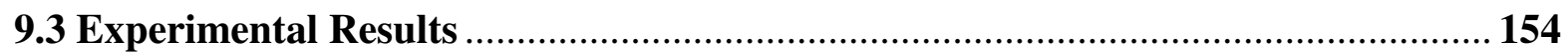

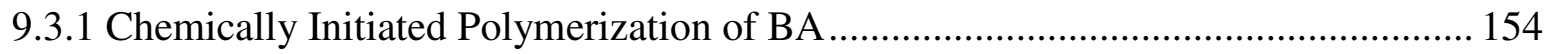

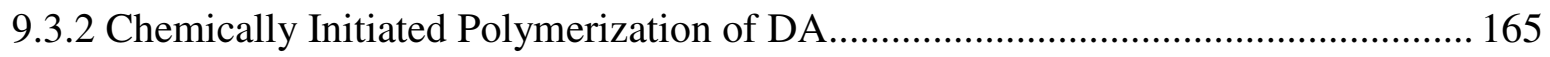

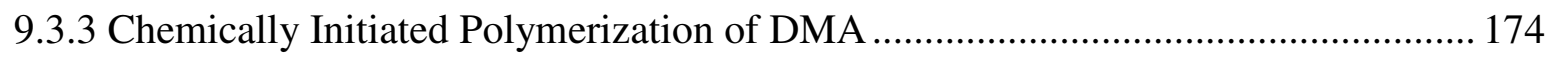

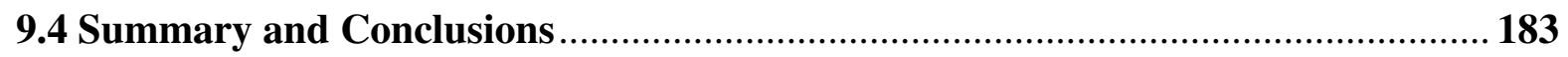

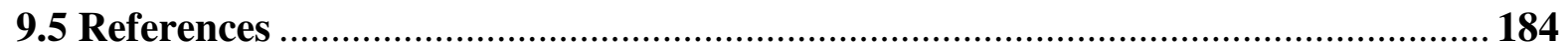

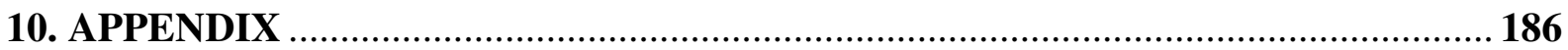




\section{Abstract}

Three aspects of free-radical polymerization have been addressed: (i) the propagation rate in acrylate/methacrylate copolymerizations, (ii) the chain-length dependence of the termination rate coefficient in bulk polymerization, and (iii) the analysis of the type of radicals produced in acrylate homopolymerizations and their influence on the reaction rate.

Propagation kinetics in free-radical bulk (co)polymerization have been investigated by applying the pulsed laser polymerization size-exclusion chromatography (PLP-SEC) technique to several binary acrylate/methacrylate systems. Copolymerization propagation rate coefficient, $k_{\mathrm{p}, \mathrm{copo}}$, and copolymer composition of the systems MA/MMA, DA/DMA, MA/DMA, and DA/MMA have been studied at 1000 bar and temperatures between $22{ }^{\circ} \mathrm{C}$ and $40{ }^{\circ} \mathrm{C}$. The systems were chosen such as to consist of acrylates and methacrylates with alkyl ester chains being of both small (methyl), both large (dodecyl) or one small and the other large. The data is analyzed in terms of the terminal and the IPUE models. In all cases the terminal model allows for good individual fits of $k_{\mathrm{p}, \text { copo }}$ and of copolymer composition. Moreover, the terminal model is capable of describing the composition and $k_{\mathrm{p}, \text { copo }}$ data with almost the same reactivity ratios for the systems DA/DMA, MA/MMA, and MA/DMA measured at 22 to $23{ }^{\circ} \mathrm{C} / 1000$ bar but failed to represent the same systems at $40{ }^{\circ} \mathrm{C} / 1000 \mathrm{bar}$. In the latter case, the IPUE model was able to fit the obtained results satisfactorily. For the system DA/MMA at both temperatures $\left(23{ }^{\circ} \mathrm{C}\right.$ and $\left.40{ }^{\circ} \mathrm{C}\right)$ even the IPUE model provides no reasonable representation of $k_{\mathrm{p}, \mathrm{copo}}$ and copolymer composition.

Single pulse initiation was combined with time-resolved electron spin resonance (ESR) detection of the decay in pulse-laser-induced radical concentration. Dodecyl methacrylate (DMA), cyclohexyl methacrylate (CHMA) and benzyl methacrylate (BzMA) were investigated via the novel single pulse-pulsed laser polymerization-electron spin resonance (SP-PLP-ESR) technique. An important advantage of SP-PLP-ESR over the conventional single pulse-pulsed laser polymerization-near-infrared spectroscopy (SP-PLP-NIR) technique is that the termination rate coefficient for two radicals of the same chain lengths, $k_{\mathrm{t}}(i, i)$, can be determined by a single differentiation of radical concentration, $c_{\mathrm{R}}(t)$, whereas monomer concentration, $c_{\mathrm{M}}(t)$, measured in SP-PLP-NIR has to be differentiated twice to yield $k_{\mathrm{t}}(i, i)$. The values of termination rate coefficient for DMA bulk polymerization show close agreement with the literature data obtained using the SP-PLP-NIR technique. The results for 
DMA, CHMA and BzMA provide strong support for the recently proposed composite model, in which termination rate coefficients, starting from small radicals, first considerably decrease with increasing free-radical size, e.g., with a power-law exponent of $\alpha_{1}=0.5$, and subsequently, e.g., at chain lengths above $i=100$, decrease to a weaker extent, e.g., with the theoretically predicted power-law exponent $\alpha_{2}=0.16$.

The ESR technique was also used for direct determination of the structure of propagating radicals in free-radical homopolymerization of $n$-butyl acrylate (BA) and dodecyl acrylate (DA) under PLP conditions (laser frequency $20 \mathrm{~Hz}$ ). The measurements were carried out in solution of toluene over a wide range of temperatures, from -50 to $70{ }^{\circ} \mathrm{C}$. At low temperature, a six-component 4-line spectrum is seen. This spectrum is assigned to a secondary propagating radical with one $\alpha$-proton and two $\beta$-methylene protons. At higher temperatures, the ESR spectrum changes to a 7-line spectrum with broader line width. The ESR spectra obtained at low degrees of monomer conversion and intermediate temperatures may be adequately represented by superimposing the six-component 4-line spectrum of secondary propagating radicals with the 7-line spectrum assigned to tertiary mid-chain radicals produced by intramolecular backbiting. The large fraction of mid-chain radicals explains the difficulties of applying the PLP-SEC technique toward $k_{\mathrm{p}}$ analysis of acrylates at temperatures of $30{ }^{\circ} \mathrm{C}$ and above.

The tertiary mid-chain radicals are rather stable. Monomer addition to such mid-chain radical proceeds at a much slower rate than addition to the parent secondary radical. In order to extend the knowledge about propagation kinetics of mid-chain radicals, the propagation rate coefficients of the dodecyl acrylate dimer (DAD) species, that forms radicals of a structure similar to the mid-chain radical, were measured as a function of temperature by applying PLP-SEC. The activation energy of the propagation reaction of DAD is obtained to be $E_{\mathrm{A}}=35.4 \pm 4.3 \mathrm{~kJ} \cdot \mathrm{mol}^{-1}$. This value is in good agreement with literature data for the methyl acrylate dimer and the butyl acrylate dimer.

Chemically initiated polymerizations of BA, DA, and DMA were carried out. In these measurements conversion as a function of time is detected and reaction orders of monomer and initiator have been deduced. In all cases a reaction order close to 0.5 is found with respect to initiator concentration. The experiments indicate that for BA and DA the reaction order with respect to monomer concentration is higher than the ideal reaction order of unity, 
whereas, the monomer reaction order for DMA is close to unity. From the steady-state freeradical polymerizations of BA, DA and DMA, the variation of the rate of polymerization and of the coupled parameter $k_{\mathrm{p}} / k_{\mathrm{t}}^{0.5}$ have been determined as a function of temperature, monomer, and initiator concentration. The kinetic behavior observed for BA and DA is assigned to the formation of mid-chain radicals. It was found that the ideal kinetic model describes DMA homopolymerization remarkably well. Termination rate coefficients for DMA obtained from the chemically initiated polymerizations, from SP-PLP-NIR, and from SP-PLPESR show close agreement. 


\section{INTRODUCTION}

The worldwide production of synthetic polymers exceeds 100 million tons per year [1]. The main route to produce polymers is free-radical polymerization, which may be performed under relatively undemanding conditions. Some typical monomers, which readily undergo free-radical polymerization, are ethylene, styrene, vinyl acetate, methyl methacrylate, methyl acrylate, etc. Polyacrylates are produced almost exclusively by radical polymerization. Conventional radical sources, e.g., peroxides or azo-compounds, are used as initiators. Methyl, ethyl, $n$-butyl, and 2-ethylhexyl acrylate are produced on a large scale. Other acrylate esters such as tert-butyl, isobutyl, or dodecyl acrylates are also produced industrially, but not on a large scale. Most homopolymers of acrylates are suitable only for a few areas of application because of extremely low glass transition temperatures. They are too soft, too tacky, or show too high elongation and insufficient strength for many applications. Polymer with specific properties may be produced by copolymerization with monomers whose homopolymers have high glass transition temperature. Methacrylates, in particular methyl methacrylate, styrene and some others are used as comonomers to obtain desired copolymer properties.

The aim of most polymerization kinetic studies has been the determination of accurate values for the individual rate coefficients governing free-radical polymerization, as the kinetics defines the structure and thus the properties of the polymer. Up to the late 1980s, propagation and termination rate coefficients were accessible only in their coupled form, $k_{\mathrm{p}} / k_{\mathrm{t}}^{0.5}$, or individually via combination of stationary and non-stationary techniques (e.g., the rotating sector method). However, differences in experimental conditions between a stationary polymerization and non-stationary polymerization lead to different radical populations and hence, because of chain-length dependence of $k_{\mathrm{t}}$, to an enormous scatter in the obtained data. The situation has dramatically improved since pulsed laser polymerization (PLP) with molecular weight analysis by size-exclusion chromatography (SEC) has been established as the method of choice to measure the propagation rate coefficients, $k_{\mathrm{p}}$, for free-radical polymerization in the late 1980 s $[2,3]$.

Applying PLP in conjunction with infrared or near-infrared (NIR) spectroscopic measurement of monomer conversion induced by a single laser pulse (SP-PLP) allows for the determination of the ratio of termination to propagation rate coefficients, $k_{\mathrm{p}} / k_{\mathrm{t}}$, in wide ranges of temperature, pressure and monomer conversion [4]. $k_{\mathrm{t}}$ is obtained by implementing $k_{\mathrm{p}}$ values 
from independent PLP-SEC experiments. The distribution of free-radical chain lengths after a single pulse, typically of $20 \mathrm{~ns}$ width, is close to a Poisson distribution with chain length $i$ being linearly correlated to time $\left(i=k_{\mathrm{p}} \cdot c_{\mathrm{M}} \cdot t\right)$, unless chain transfer interferes. As a consequence, SP-PLP experiments may provide access to investigations into the chain-length dependence of $k_{\mathrm{t}}$. The measurement of $k_{\mathrm{t}}$ via SP-PLP is indirect in that the time-dependence of monomer concentration, rather than that of radical concentration, is measured.

From theory it is known that the electron spin resonance (ESR) technique can provide information on both the structure and the concentration of propagating radicals [5]. Accordingly, ESR spectroscopy is a promising method for obtaining information on paramagnetic species (free radicals) in radical polymerization systems.

In the present work the principles of single pulse-pulsed laser polymerization were combined with direct monitoring of the radical decay after applying a laser pulse via online timeresolved ESR spectroscopy. Time-resolved measurements of macroradical concentration, $c_{\mathrm{R}}$, via ESR spectroscopy were first carried out by Westmoreland et al. [6] and by Zhu et al. [7]. Westmoreland et al. measured $c_{\mathrm{R}}$ during high-conversion semi-continuous emulsion copolymerization processes. Non-stationary conditions were achieved by shutting down the flow of a red-ox initiator. Absolute radical concentration was determined from ESR peak heights. Zhu et al. carried out time-resolved ESR measurements on methyl methacrylate bulk polymerizations at $25^{\circ} \mathrm{C}$ under "post-effect" conditions, which were created by switching off the UV lamp used for inducing AIBN decomposition. The decay in radical concentration was monitored by measuring the height of the central line of the ESR spectrum.

The ESR studies into $k_{\mathrm{t}}$ carried out in those studies were limited in that: $(i)$ initiation could not be instantaneously stopped and (ii) the chain-length distribution of radicals at $t=0$ (that is, at the moment when either photoinitiation or the addition of an initiator ceased) was broad. These limitations may be overcome by using time-resolved ESR spectroscopy in conjunction with initiation by a single laser pulse, in which case primary radicals are produced (almost) instantaneously. There is no initiation in the dark-time period as initiation starts sharply at $t=0$. Moreover, the free-radical chain-length distribution is close to monodisperse (of Poisson-type) throughout the entire experiment unless chain transfer processes come into play.

To distinguish the novel technique from the existing SP-PLP method, it will be referred to as SP-PLP-ESR whereas the established method will be referred to as SP-PLP-NIR.

SP-PLP-ESR experiments provide access to measuring the chain-length dependence of $k_{\mathrm{t}}$ by analyzing the rate of radical disappearance at different times $t$ after applying a laser pulse. 
The SP-PLP-ESR method is, however, not without difficulties. A major problem is associated with the time-resolved measurement of radical concentrations in the range $10^{-6}$ to $10^{-8} \mathrm{~mol} \cdot \mathrm{L}^{-1}$. According to the SP-PLP relation $t=i /\left(k_{\mathrm{p}} \cdot c_{\mathrm{M}}\right)$, rapidly propagating monomers require highly time-resolved experiments. E.g., with acrylate monomers, where $k_{\mathrm{p}}$ is of the order of $10^{5} \mathrm{~L} \cdot \mathrm{mol}^{-1} \cdot \mathrm{s}^{-1}$, it takes approximately 10 microseconds for one propagation step to occur. High termination rate also requires high experimental time resolution. Thus SP-PLPESR studies are easier applied to low $k_{\mathrm{p}}$ - low $k_{\mathrm{t}}$ monomers.

Within the present work acrylate/methacrylate copolymerization systems were measured. It is

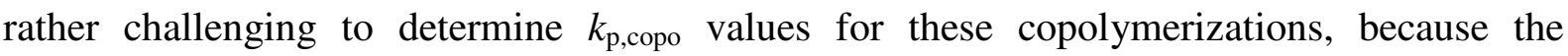
corresponding homopolymerization propagation rate coefficients differ considerably.

Moreover, a general problem with acrylates is related to the observation that at temperatures above $30{ }^{\circ} \mathrm{C}$, the PLP-SEC technique results in a broad molecular weight distribution, which does not show the characteristics of a successful PLP-experiment. For this reason the $k_{\mathrm{p}, \text { copo }}$ data was determined for acrylate/methacrylate copolymerizations at $22-23{ }^{\circ} \mathrm{C} / 1000 \mathrm{bar}$.

It is assumed, that the difficulty in obtaining reliable $k_{\mathrm{p}}$ values for acrylates at temperatures above $30{ }^{\circ} \mathrm{C}$ is caused by the effect of intramolecular chain transfer to polymer (backbiting). Resulting tertiary radicals are relatively stable with a significant steric hindrance contributing to the low addition rate to monomer of these species [8]. The occurrence of mid-chain radicals, produced by transfer-to-polymer processes, has been confirmed by ESR spectroscopy under stationary free-radical polymerization conditions chiefly carried out by Yamada and colleagues [9,10,11], by Kajiwara and Kamachi [12], and by the observation of quaternary carbon atoms with ${ }^{13} \mathrm{C}-\mathrm{NMR}$ under PLP conditions [13]. PLP experiments on BA and DA carried out in conjunction with ESR detection should allow for identifying the amounts of secondary propagating and mid-chain radical concentrations in a wide temperature range.

Finally, modeling of chemically initiated polymerization of BA, DA and DMA will be performed. 


\subsection{REFERENCES}

[1] Industrial Polymers Handbook, Ed. by Wilks, E. S.; Wiley-VCH Verlag GmbH: Weinheim, 2001.

[2] Olaj, O. F.; Bitai, I.; Hinkelmann, F. Macromol. Chem. 1987, 188, 1689.

[3] Olaj, O.F.; Schnöll-Bitai, I. Eur. Polym. J. 1989, 25, 635.

[4] Buback, M.; Hippler, H.; Schweer, J.; Vögele, H.-P. Makromol. Chem., Rapid Commun. 1986, 7, 261.

[5] ESR Spectroscopy in Polymer Research, Randy, B.; Rabek, J.F.; Springer-Verlag: Berlin, 1977.

[6] Westmoreland, D. G.; Lau, W. Macromolecules 1989, 22, 496.

[7] Zhu, S.; Tian, Y.; Hamielec, A. E. Macromolecules 1990, 23, 1144.

[8] Asua, J. M.; Beuermann, S.; Buback, M.; Castignolles, P.; Charleux, B.; Gilbert, R. G.; Hutchinson, R. A.; Leiza, J. R.; Nikitin, A. N.; Vairon, J. P.; Herk, A. M. v. Macromol. Chem. Phys. 2004, 205, 2151.

[9] Azukizawa, M.; Yamada, B.; Hill, D. J. T.; Pomery, P. J. Macromol. Chem. Phys. 2000, 201, 774 .

[10] Sato, E.; Emoto, T.; Zetterlund, P. B.; Yamada, B. Macromol. Chem. Phys. 2004, 205, 1829.

[11] Yamada, B.; Azukizawa, M.; Yamazoe, H.; Hill, D. J. T.; Pomery, P. J. Polymer 2000, $41,5611$.

[12] Kajiwara, A.; Kamachi, M. ACS Symposium Series 2003, 854 (Advances in Controlled/Living Radical Polymerization), 86.

[13] Plessis, C.; Arzamendi, G.; Alberdi, J. M.; van Herk, A. M.; Leiza, J. R.; Asua, J. M. Macromol. Rapid Commun. 2003, 24, 173. 


\section{THEORETICAL BACKGROUND}

\subsection{Ideal Polymerization Kinetics}

Ideal polymerization kinetics is based on four assumptions:

- all reactions are irreversible

- monomeric species are only consumed in propagation steps

- all macroradicals show the same reactivity, irrespective of their chain length

- termination takes place only by disproportionation or bimolecular radical combination

By these assumptions a kinetic scheme of a free-radical polymerization can be characterized with three fundamental steps: the formation of radicals, propagation of these radicals and termination of the radical chains.

Each reaction will be described in more detail below.

\subsubsection{INITIATION}

The formation of radicals can take place after thermal, chemical or photochemical activation of an initiator. It may also be possible to induce polymerization by directly exciting the monomer. In this work, initiation by chemical and photochemical decomposition of an initiator is used.

$$
\mathrm{I} \stackrel{k_{\mathrm{d}}}{\longrightarrow} 2 \mathrm{R}
$$

In case of photochemical induced polymerization a UV laser pulse of approximately $20 \mathrm{~ns}$ duration is fired on the reaction mixture (containing the initiator and the monomer(s)), with the intention of inducing an instantaneous free-radical population. The formation of radicals is thus fast in comparison to termination and propagation processes. The radical concentration, which is generated by a single laser pulse, $c_{\mathrm{R}}{ }^{0}$, is given by Eq. 3.1

$$
c_{\mathrm{R}}^{0}=2 \cdot \Phi \cdot \frac{n_{\mathrm{abs}}}{V}
$$

where $\Phi$ is the primary quantum yield, $n_{\text {abs }}$ is the number of absorbed photons and $V$ is the irradiated volume. The primary quantum yield is the product of the laser efficiency $\varphi$ and the 
initiator efficiency $f$. According to Beer-Lambert's law, the number of absorbed photons can be calculated by Eq. 3.2.

$$
n_{\mathrm{abs}}=\frac{E_{\mathrm{P}}}{E_{\lambda}} \cdot\left(1-10^{-\varepsilon \cdot c_{1} \cdot l}\right)
$$

$E_{\mathrm{P}}: \quad$ energy of one laser pulse

$E_{\lambda}: \quad$ energy of one mole of photons at the laser wavelength $\lambda$

$\varepsilon$ : molar absorption coefficient of the initiator molecule at the laser wavelength $\lambda$

$c_{\mathrm{I}}: \quad$ photoinitiator concentration

$l$ : $\quad$ optical path length

The effective rate of initiation in chemically initiated polymerization is given by:

$$
v_{\mathrm{in}}=\frac{\mathrm{d} c_{\mathrm{R}, \mathrm{I}}}{\mathrm{d} t}=2 \cdot k_{\mathrm{d}} \cdot f \cdot c_{\mathrm{I}}
$$

where $c_{\mathrm{R}, \mathrm{I}}$ is the concentration of radicals of an initiator, $k_{\mathrm{d}}$ is the rate coefficient of initiator decomposition, $f$ is the initiator efficiency and $c_{\mathrm{I}}$ is the initiator concentration.

\subsubsection{Propagation}

During the propagation step a monomer molecule is added to a macroradical.

$$
\mathrm{R} \cdot+\mathrm{M} \cdot \stackrel{k_{\mathrm{P}}}{\longrightarrow} \mathrm{R}
$$

The change in monomer concentration can be expressed by the following rate law:

$$
\frac{\mathrm{d} c_{\mathrm{M}}}{\mathrm{d} t}=-k_{\mathrm{p}} \cdot c_{\mathrm{M}} \cdot c_{\mathrm{R}}
$$

$k_{\mathrm{p}}: \quad$ propagation rate coefficient

$c_{\mathrm{R}}$ : macroradical concentration

$c_{\mathrm{M}}: \quad$ monomer concentration

In the case of copolymerization, $c_{\mathrm{M}}$ is the overall monomer concentration. 


\subsubsection{TERMINATION}

The termination reaction proceeds either by disproportionation, which involves the transfer of a $\beta$-hydrogen from one radical to the other, or by combination, which is usually a simple head to head coupling of the radicals involved.

$$
\begin{gathered}
\mathrm{R}_{\mathrm{n}} \cdot+\mathrm{R}_{\mathrm{m}} \cdot \stackrel{k_{\mathrm{t}, \mathrm{d}}}{\longrightarrow} \mathrm{P}_{\mathrm{n}}+\mathrm{P}_{\mathrm{m}} \\
\mathrm{R}_{\mathrm{n} \cdot} \cdot+\mathrm{R}_{\mathrm{m}} \cdot \stackrel{k_{\mathrm{t}, \mathrm{c}}}{\longrightarrow} \mathrm{P}_{\mathrm{n}+\mathrm{m}}
\end{gathered}
$$

The termination rate coefficient, $k_{\mathrm{t}}$, is the sum of the rate coefficient for disproportionation, $k_{\mathrm{t}, \mathrm{d}}$, and the rate coefficient for combination, $k_{\mathrm{t}, \mathrm{c}}$. In the termination step the actual dead polymer chains are formed. The rate of termination is second order in the radical concentration $c_{\mathrm{R}}$ :

$$
\frac{\mathrm{d} c_{\mathrm{R}}}{\mathrm{d} t}=-2 \cdot k_{\mathrm{t}} \cdot c_{\mathrm{R}}^{2}
$$

in which $k_{\mathrm{t}}$ is the termination rate coefficient of two radicals. Integration of Eq. 3.5 yields Eq. 3.6

$$
\frac{c_{\mathrm{R}}(t)}{c_{\mathrm{R}}^{0}}=\left(1+2 \cdot k_{\mathrm{t}} \cdot c_{\mathrm{R}}^{0} \cdot t\right)^{-1}
$$

Substitution of Eq. 3.6 into Eq. 3.4 yields the change in relative monomer concentration after a single laser pulse:

$$
\frac{\mathrm{d} c_{\mathrm{M}}}{\mathrm{d} t}=-k_{\mathrm{p}} \cdot c_{\mathrm{M}} \cdot\left(2 \cdot k_{\mathrm{t}} \cdot t+\frac{1}{c_{\mathrm{R}}^{0}}\right)^{-1}
$$

By integration from this, one obtains the monomer concentration as a function of time:

$$
\frac{c_{\mathrm{M}}(t)}{c_{\mathrm{M}}^{0}}=\left(2 \cdot k_{\mathrm{t}} \cdot c_{\mathrm{R}}^{0} \cdot t+1\right)^{-\frac{k_{\mathrm{p}}}{2 \cdot k_{\mathrm{t}}}}
$$

$c_{\mathrm{M}}{ }^{0}: \quad$ initial monomer concentration prior to the laser pulse

$c_{\mathrm{R}}{ }^{0}$ : initial free-radical concentration generated by a laser pulse

Chemical reaction rates usually are close to the diffusion controlled limit [1]. Especially in a polymerization, where the termination step involves the diffusion of two macroradicals towards each other before the actual reaction can take place diffusion control is found [2]. The process of termination can be divided into three stages: first translational (or center-ofmass) diffusion of the center of gravity of two radicals should occur resulting in collision of 
the two coiled growing polymer chains after which segmental diffusion has to take place in which the two radical chain ends of the entangled macroradicals terminate approaching each other.

To describe termination processes, a third type of diffusion may be invoked: reaction diffusion. This is a process in which the addition of a monomer unit causes a displacement of a radical chain end. At very high viscosities, when the entire chain is almost immobile, this may prevent the rate determining process for radical ends to approach each other.

The fact that for polymerization termination is diffusion controlled, makes it dependent on several parameters with respect to the medium, especially the viscosity. Furthermore, as chains grow longer, these chains will diffuse more slowly through the medium. As a consequence, the rate coefficient of termination is thought to be chain length dependent. It is generally accepted that the termination rate coefficient depends on the following factors [3]: (1) viscosity of the polymerizing system, (2) chain length of the terminating radicals, (3) temperature, (4) pressure, and (5) monomer conversion.

In section 6, the chain-length dependence of $k_{\mathrm{t}}$ will be discussed.

\subsubsection{Chain TRANSFER}

During polymerization, transfer of the radical functionality can occur to monomer, initiator, polymer, solvent or to transfer agents.

$$
\mathrm{R} \cdot+\mathrm{X} \stackrel{k_{\mathrm{tr}, \mathrm{X}}}{\longrightarrow} \mathrm{P}+\mathrm{X}
$$

The rate of chain transfer to a species $\mathrm{X}$ is given by:

$$
\frac{\mathrm{d} c_{\mathrm{X}}}{\mathrm{d} t}=-k_{\mathrm{tr}, \mathrm{X}} \cdot c_{\mathrm{R}} \cdot c_{\mathrm{X}}
$$

where $k_{\mathrm{tr}, \mathrm{X}}$ is the rate coefficient of chain transfer to a species $\mathrm{X}$ and $c_{\mathrm{X}}$ is the concentration of this species $\mathrm{X}$, acting as chain transfer agent. A combined parameter, the rate constant $C_{\mathrm{tr}, \mathrm{X}}$, is used to describe transfer. This parameter is the ratio of the rate coefficients of transfer to propagation:

$$
C_{\mathrm{tr}, \mathrm{X}}=\frac{k_{\mathrm{tr}, \mathrm{X}}}{k_{\mathrm{p}}}
$$


This chain transfer step can have a large influence on molecular weight distribution, usually by reducing molecular weights.

\subsubsection{OVERALL REACTION}

In a chemically initiated polymerization the radical concentration is assumed to be quasistationary. The overall polymerization rate, $R_{\mathrm{p}}$, under ideal conditions is given by the following equation:

$$
R_{\mathrm{p}}=-\frac{\mathrm{d} c_{\mathrm{M}}}{\mathrm{d} t}=\frac{k_{\mathrm{p}}}{\sqrt{k_{\mathrm{t}}}} \cdot c_{\mathrm{M}} \cdot \sqrt{v_{\mathrm{in}}}
$$

\subsection{Determination of Propagation and Termination Rate COEFFICIENTS}

Up to the late 1980 s, termination and propagation rate coefficients were accessible only in their coupled form, $k_{\mathrm{p}} / k_{\mathrm{t}}^{0.5}$, or individually via combination with non-stationary techniques such as the rotating sector or spatially intermittent polymerization methods in combination with stationary polymerization measurements. The only exception has been the direct determination of the propagation rate coefficients via the measurement of the steady-state free-radical concentration by ESR experiments in combination with rate measurements. However, these measurements have always been associated with some difficulties. The situation has dramatically improved with the invention of the pulsed laser initiated polymerization technique (PLP) in the late 1980s [4,5]. Since then, this technique in conjunction with subsequent analysis of molecular weight distributions of resulting polymers by size-exclusion chromatography (SEC) has been extensively used to obtain propagation rate coefficients for various homo- and copolymerizations. Today, the PLP-SEC method is almost exclusively used for determination of propagation rate coefficients and has been recommended by the IUPAC for the measurement of $k_{\mathrm{p}}$. Applying PLP in conjunction with infrared or near-infrared (NIR) spectroscopic measurement of monomer conversion induced by a single laser pulse (SP-PLP-NIR) allows for determination of the ratio of termination to propagation coefficients, $k_{\mathrm{t}} / k_{\mathrm{p}}$, in wide ranges of temperature, pressure, and monomer conversion [6]. SP-PLP experiments may provide access to investigations onto the chainlength dependence of $k_{\mathrm{t}}$ (see section 6). 


\subsubsection{Photoinitiator DeComposition}

The photoinitiator used in the ideal pulsed laser polymerization experiments should fulfill a couple of requirements. In the past these requirements have been defined as follows [7]:

- The photoinitiator should decompose upon irradiation with excimer laser light. A wavelength of $351 \mathrm{~nm}$ is preferable, because most monomers do not absorb at this wavelength.

- Laser-induced photoinitiator decomposition must be fast as compared to the subsequent first propagation step (below one microsecond).

- The efficiency of the initiator should be high, preferably close to one, which says that all radicals generated start a growing chain.

- Both radical fragments should be capable of rapidly initiating macromolecular growth, which ensures close to monodisperse size distribution of growing radicals.

It was shown that 2,2-dimethoxy-2-phenylacetophenone (DMPA) cannot fulfill all these requirements [8,9]. This photoinitiator decomposes into two radical species, $R_{1}$ and $R_{2}$ (see for example [10]):<smiles>COC(OC)(C(=O)c1ccccc1)c1ccccc1</smiles>

DMPA

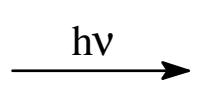

$\mathrm{R}_{1}$<smiles>O=Cc1ccccc1</smiles><smiles>COC(=O)c1ccccc1</smiles>

$\mathrm{R}_{2}$

Both species are distinctly different in their character. Whereas a benzoyl radical $\mathrm{R}_{1}$ is highly efficient in adding to monomer, an acetal radical $\mathrm{R}_{2}$ does not noticeably add to monomer in the dark time period after the pulse, but may react with radicals and thus behaves like an inhibitor species. The poor propagating activity of $\mathrm{R}_{2}$ has first been described by the Fischer group [10] and has recently been demonstrated through MALDI experiments by the Davis group [11]. The simultaneous initiation and inhibition activity of the DMPA-derived species 
results in a rather peculiar SP-PLP behavior: The monomer conversion vs. time traces measured at different initial DMPA contents, but otherwise identical reaction conditions, intersect each other [12]. This crossing behavior provides access to measuring the chainlength dependence of the termination rate coefficient [12]. Measuring several SP-PLP traces under conditions where are all reaction conditions except DMPA concentration are identical, however, becomes increasingly difficult toward moderate and high degrees of monomer conversion. For this reason, it is highly desirable to use an ideal photoinitiator which decomposes into two free-radical fragments both of which easily add to a monomer molecule. Such a photoinitiator allows for deducing the chain-length dependence of termination rate coefficients from a single SP-PLP trace.

Investigations by Külpmann into several photoinitiators revealed that 2-methyl-4(methylthio)-2-morpholino-propiophenone (MMMP), is a close-to-ideal photoinitiator which rapidly decomposes into two propagating free-radical species $[13,14]$. The scheme of the MMMP decomposition is given below:

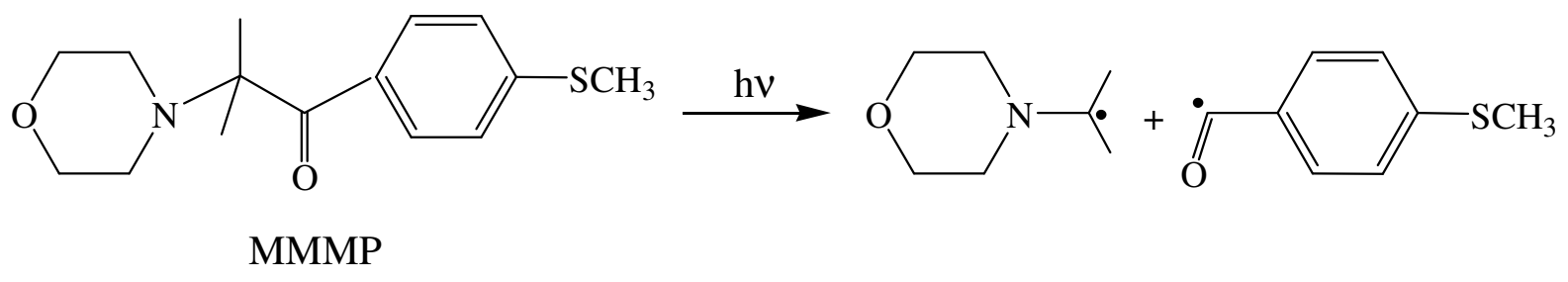

MMMP was used in all experiments for determination of $k_{\mathrm{t}}(i, i)$. The measurements have been carried out within extended ranges of monomer conversion.

\subsubsection{Determination of the Propagation Rate Coefficient, $k_{\mathrm{p}}$, With the Pulsed LASER METHOD}

Pulsed laser techniques have enormously improved the quality by which rate coefficients of individual steps in free-radical polymerization may be measured. Pulsed laser initiated polymerization (PLP) in conjunction with size-exclusion chromatography (SEC) yields the propagation rate coefficient, $k_{\mathrm{p}}$. Aspects of PLP along these lines were first put forward by Aleksandrov et al. [15]. It is, however, the pioneering work of Olaj and coworkers [16,17] by which the application of pulsed laser techniques toward $k_{\mathrm{p}}$ measurement was demonstrated. In PLP-SEC, the pulsed laser light almost instantaneously creates an intense burst of freeradicals. Varying the laser pulse repetition rate allows for producing generations of primary 
free-radicals at pre-selected time intervals. The average time necessary for each single propagation step is the inverse of the propagation frequency. This frequency is given by $k_{\mathrm{p}} \cdot c_{\mathrm{M}}$. With a time $t_{0}$ between two pulses available for growth, a chain length $L_{0}$ is expected for the polymer chains, given by the simple equation:

$$
L_{0}=k_{\mathrm{p}} \cdot c_{\mathrm{M}} \cdot t_{0}
$$

The pulsing translates into a characteristic pattern of the molecular weight distribution (MWD). Analysis of the MWD enables the unambiguous determination of the propagation rate coefficient, $k_{\mathrm{p}}$. Olaj et al. [16] have demonstrated that the inflection point at the lowmolecular-weight side of the peak is the best measure of $L_{0}$. This point may be determined by locating the maxima of the first derivative curve of the MWD.

For copolymerizations an average value of $k_{\text {p,copo }}$ can be determined using the same equation as for homopolymerizations (Eq. 3.12) with two exceptions, namely: (I) an average monomer concentration $\overline{c_{\mathrm{M}}}$ and (II) an average chain length $\bar{L}$ are used:

$$
\begin{gathered}
\overline{c_{\mathrm{M}}}=\frac{n_{\text {total }}}{V_{\text {total }}}=\frac{n_{1}+n_{2}}{V_{1}+V_{2}}=\frac{\frac{m_{1}}{M_{1}}+\frac{m_{2}}{M_{2}}}{\frac{m_{1}}{\rho_{1}}+\frac{m_{2}}{\rho_{2}}} \\
\bar{L}=\frac{M_{W P}}{M_{1} \cdot F_{1}+M_{2} \cdot F_{2}}
\end{gathered}
$$

where $n_{\text {total }}$ is the total amount, $V_{\text {total }}$ is the total volume of the solution; $n_{\mathrm{i}}$ is the amount, $m_{\mathrm{i}}$ is the mass, $M_{\mathrm{i}}$ is the molecular mass, $F_{\mathrm{i}}$ is the mole fraction of component $\mathrm{i}$ in a binary copolymer, and $\rho_{1}$ is the density of the pure monomer $\mathrm{i}(\mathrm{i}=1,2)$ at the investigation conditions.

\subsubsection{Determination OF THE MOLECUlaR Weight Distribution}

The reliable method for determination of the molecular weight distribution (MWD) is sizeexclusion chromatography (SEC).

During a SEC separation, polymer coils are passed through columns with highly porous material with a certain pore size distribution. Separation is based on the hydrodynamic volume of the polymer coil. Lower molecular weight material, comprising smaller coils, will spend more time in the pores than higher molecular weight material, therefore eluting more slowly through the column. After separation, the relative concentration of the eluted chains can be detected by measuring changes in, for example, the absorption (UV/IR) and the 
refractive index. For concentration-sensitive detectors, the SEC method is relative, in that a calibration curve is needed which correlates elution volume with molecular weight. The calibration curve can be constructed with calibration standards of known molecular weight of the same polymer material.

Unfortunately, calibration standards are available only for a limited number of polymers. Without such standards present, the distributions can be calculated using the principle of universal calibration [18]. For a dissolved random coil macromolecule, a scaling is assumed between the hydrodynamic volume and the molecular weight [19], according to:

$$
H V=\frac{[\eta] \cdot M}{2.5 N_{\mathrm{Av}}}
$$

with $H V$ the hydrodynamic volume, $[\eta]$ the intrinsic viscosity and $N_{\text {av }}$ Avogadro's number. The most often used relation between viscosity and molecular weight is given by the MarkHouwink relation [20,21], which assumed a linearity between $\log [\eta]$ and $\log M$ according to

$$
[\eta]=K \cdot M^{a}
$$

with $K$ and $a$ being the Mark-Houwink parameters. In most cases, the Mark-Houwink parameters are known and these are used for universal calibration. First, a calibration curve relating $\log M_{1}$ to the elution volume is made. Then the assumption is made that at each elution volume the hydrodynamic volume is the same irrespective of the type of polymer. The calibration curve can then be computed for a polymer other than the polymer used for calibration from the following equation, using Eq. 3.15 and 3.16:

$$
\log M_{2}=\frac{1}{1+a_{2}} \log \frac{K_{1}}{K_{2}}+\frac{1+a_{1}}{1+a_{2}} \log M_{1}
$$

with the index 1 indicating the polymer used for calibration and the index 2 for the unknown polymer.

An alternative has become available for situations where calibration standards of MarkHouwink parameters are not available for a certain polymer. This consists of coupling mass sensitive detectors for SEC. The detector response of such a detector is not only dependent on the polymer concentration, but also on a polymer property that is related to the molecular weight of the analyzed polymer. Such mass sensitivity detectors include light scattering techniques [22] and on-line viscometry [23] or a combination of both methods [24]. A SEC setup consisting of both mass sensitive detectors and a concentration detector is often referred to as a triple detector setup. As light scattering and viscosity both are absolute functions of molecular weight, the need for standards of Mark-Houwink parameters can be overcome. 


\subsubsection{Determination of the Coupled Parameters $k_{\mathrm{p}} \cdot \boldsymbol{k}_{\mathrm{t}}^{-0.5}$ From Chemically INITIATED POLYMERIZATION}

For the determination of the coupled parameters $k_{\mathrm{p}} / k_{\mathrm{t}}^{0.5}$ from a chemically initiated polymerization, monomer concentration during the polymerization reaction is measured via NIR spectroscopy. From one set of spectroscopic measurements the dependence of monomer concentration on time can be determined.

Assuming that the reaction obeys ideal kinetics, the coupled parameters $k_{\mathrm{p}} / k_{\mathrm{t}}^{0.5}$ can be derived from Eq. 3.11, if the efficiency of an initiator $f$ and the rate coefficient of initiator decomposition $k_{\mathrm{d}}$ are known or can be estimated. The actual initiator concentration $c_{\mathrm{I}}$ is obtained from the integrated form of the initiator decomposition rate law.

The individual termination rate coefficient $k_{\mathrm{t}}$ is calculated from $k_{\mathrm{p}} / k_{\mathrm{t}}^{0.5}$, with $k_{\mathrm{p}}$ been taken from independent PLP-SEC experiments.

\subsubsection{Determination OF THE Termination RATE Coefficient, $k_{t}$, From THE SP-PLP-NIR EXPERIMENTS}

The monomer conversion induced by a laser pulse is measured as a function of time with $\mu \mathrm{s}$ time resolution. Conversion has been monitored by the increase of polymer absorption in the first overtone region of the $\mathrm{C}-\mathrm{H}$ stretching modes around $6170 \mathrm{~cm}^{-1}$. The time resolution of NIR spectroscopic measurement of polymer concentration is close to $1 \mu \mathrm{s}$ [25].

Eq. 3.8 represents the time evolution of relative monomer concentration within a single pulsepulsed laser polymerization (SP-PLP) experiment. In the absence of any chain-length dependence of $k_{\mathrm{t}}$ (and of $k_{\mathrm{p}}$ ), fitting the experimental vs. time trace to Eq. 3.8 immediately yields $k_{\mathrm{t}} / k_{\mathrm{p}}$ and $k_{\mathrm{t}} \cdot c_{\mathrm{R}}{ }^{0}$. As $c_{\mathrm{R}}{ }^{0}$ is not directly accessible from SP-PLP-NIR measurements, the primary experimental quantity from SP-PLP-NIR is $k_{\mathrm{t}} / k_{\mathrm{p}} . k_{\mathrm{t}}$ is obtained by implementing $k_{\mathrm{p}}$ values from independent PLP-SEC experiments [26,27].

Whereas, the assumption that $k_{\mathrm{p}}$ is chain-length independent, is a good one at least chain length above $i=10$, the chain-length dependence of $k_{\mathrm{t}}$ cannot be ignored. As the chain-length varies with time, the rate coefficient which results from fitting of the conversion vs. time trace to Eq. 3.8 should be referred to as $<k_{\mathrm{t}}>$. 


\subsubsection{Determination OF THE Termination RATE COEFficient, $k_{t}$, From THE SP-PLP-ESR EXPERIMENTS}

Within the SP-PLP-ESR (single pulse-pulsed laser polymerization-electron spin resonance) method, photoinitiator-derived primary radicals are instantaneously generated by an excimer laser pulse and the concentration of (macro)radicals, $c_{\mathrm{R}}(t)$, is determined via online timeresolved ESR spectroscopy. Deducing $k_{\mathrm{t}}$ directly from the time dependence of radical concentration, $c_{\mathrm{R}}(t)$, constitutes an important advantage of SP-PLP-ESR.

Another advantage of SP-PLP-ESR is that $k_{\mathrm{t}}(i, i)$ can be determined by a single differentiation of $c_{\mathrm{R}}(t)$ (Eq. 3.6), whereas $c_{\mathrm{M}}(t)$ data from SP-PLP-NIR needs to be differentiated twice to yield $k_{\mathrm{t}}$. As the dependence of $k_{\mathrm{p}}$ on chain length will be restricted to lower values up to $i=10$, it seems justified to use constant $k_{\mathrm{p}}$ for modeling the extended chain length region up to $i=1000$, with $i=k_{\mathrm{p}} \cdot c_{\mathrm{M}} \cdot t$. 


\subsection{REFERENCES}

[1] Moad, G.; Solomon, D.H. The chemistry of Free Radical Polymerization, Elsevier, Oxford 1995, 28.

[2] O’Driscoll, K.F.; Mahabadi, H.K. Comprehensive Polymer Science, Pergamon Press, New York 1989, 3, 85.

[3] Handbook of Radical Polymerization, Matyjaszewski, K.; Davis, T. P., Eds.; WileyInterscience: New York, 2002.

[4] Olaj, O.F.; Schnöll-Bitai, I. Eur. Polym. J. 1989, 25, 635.

[5] Olaj, O. F.; Bitai, I.; Hinkelmann, F. Macromol. Chem. 1987, 188, 1689.

[6] Buback, M.; Hippler, H.; Schweer, J.; Vögele, H.-P. Makromol. Chem., Rapid Commun. 1986, 7, 261.

[7] Kurz, C. Ph. D. Thesis, Göttingen, 1995.

[8] Kowollik, C. Ph. D. Thesis, Göttingen, 1999.

[9] Feldermann, A. Ph. D. Thesis, Göttingen, 2003.

[10] Fischer, H.; Baer, R.; Hany, R.; Verhoolen, I.; Walbiner, M. J. Chem. Soc., Perkin Trans. 1990, 2, 787.

[11] Vana, P; Davis, T.P.; Barner-Kowollik, C. J. Polym. Sci., Part A: Polym. Chem. 2002, 40,675 .

[12] Buback, M.; Busch, M.; Kowollik, C. Macromol. Theory Simul. 2000, 9, 442.

[13] Külpmann, A. Dipl. Thesis, Göttingen, 2000.

[14] Vana, P.; Davis, T. P.; Barner.Kowollik, C. Macromol. Rapid. Commun. 2002, 23, 952.

[15] Aleksandrov, A.P.; Genkin, V.N.; Kitai, M.S.; Smirnova, I.M.; Sokolov, V.V. Sov. J. Quant Electron. 1977, 5, 547.

[16] Olaj, O.F.; Bitai, I.; Hinkelmann, F. Macromol. Chem. 1987, 188, 1689.

[17] Olaj, O.F.; Schnöll-Bitai, I. Eur. Polym. J. 1989, 25, 635.

[18] Grubisic, Z.; Rempp, P.; Benoit, H. J. Polym. Sci., Polym. Lett. 1967, 5, 753.

[19] Flory, P.J. J. Chem. Phys. 1945, 13, 453.

[20] Mark, H. in 'Der feste Körper', Hirzel, Leipzig,. 1938, 65.

[21] Houwink, R. J. Prakt. Chem. 1940, 157,15.

[22] Wyatt, P.J. Anal. Chim. Acta. 1993, 272, 1.

[23] Lesec, J.; Millequant, M.; Havard, T. in 'ACS Symposium Series', ed. Provder, T.; American Chemical Society, Washington. 1993, 521, 220. 
[24] Reed, W.F. Macromol. Chem. Phys. 1995, 196, 1539.

[25] Beuermann, S.; Buback, M. Prog. Polym. Sci. 2002, 27, 191.

[26] Buback, M.; Gilbert, R. G.; Hutchinson, R. A.; Klumperman, B.; Kuchta, F.-D.; Manders, B. G.; O’Driscoll, K. F.; Russell, G. T.; Schweer, J. Macromol. Chem. Phys. 1995, 196, 3267.

[27] Beuermann, S.; Buback, M.; Davis, T. P.; Gilbert, R. G.; Hutchinson, R. A.; Olaj, O. F.; Russell, G. T.; Schweer, J.; van Herk, A. M. Macromol. Chem. Phys. 1997, 198, 1545. 


\section{EXPERIMENTAL}

\subsection{Apparatus}

\subsubsection{Optical High-Pressure Cell}

The optical high-pressure cell used for spectroscopic investigations of pulsed laser induced homo- and copolymerizations under high pressure is illustrated in Fig. 4.1. The cell is designed for pressures up to 3500 bar and temperatures up to $350{ }^{\circ} \mathrm{C}$. The cylindrical cell body and the sealing flanges are made from a nickel-based alloy of high ultimate tensile strength (RGT 601, Material No. 2.4668, Arbed Saarstahl). The length of the cell body is $100 \mathrm{~mm}$ and the outer and inner diameters are 80 and $22 \mathrm{~mm}$, respectively. Four borings perpendicular to the cylindrical axis allow for fitting high-pressure capillaries and a sheathed thermocouple (6) directly into the sample volume.

The cell is sealed at each end by a conical ram (5) (Material No. 2.4668, Arbed Saarstahl). The ram is pressed into the cell cone by the flange (2) (Material No. 2.4668, Saarstahl) which is secured by six high-pressure bolts (1) (Material No. 2.4969). The optical path length way be varied by using different types of rams. The experiments were performed using an internal cell. The rams were chosen such that a path length of approximately 1-2 mm resulted. This allowed a sufficient cavity for the fitting of the internal cell between the two high-pressure windows. 


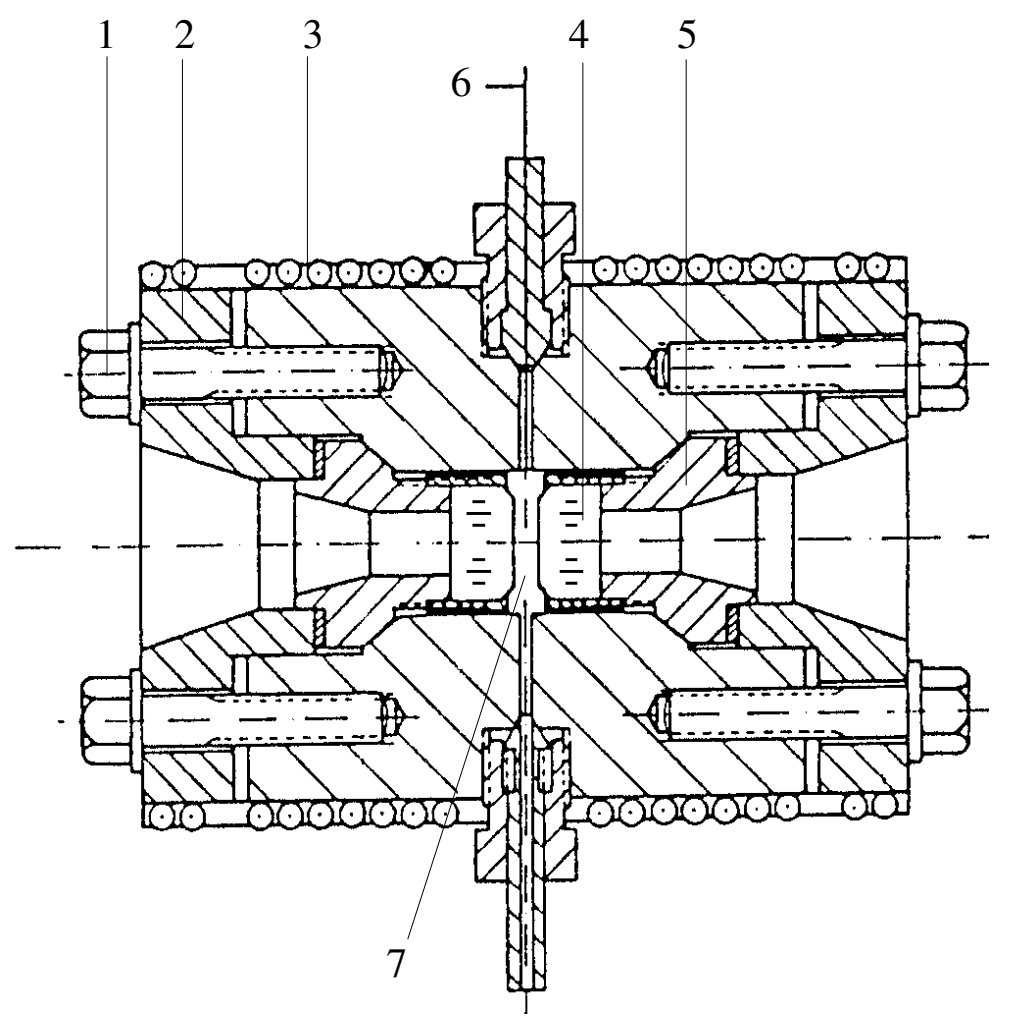

(1) bolt

(5) ram

(2) flange

(6) sheathed thermocouple

(3) heating jacket

(7) adjustable optical path length

(4) high pressure window

Fig. 4.1 Optical high pressure cell

Each high pressure window (4) is fitted against the polished surface of a ram and held in place by a stainless steel sealing cap. To compensate for surface area irregularities, a $\sim 12 \mu \mathrm{m}$ thick teflon foil is placed between the polished surface of the window and the ram. This setup is self-sealing under pressure according to the Poulter principle [1]. The high-pressure optical windows used in this work were made from synthetic sapphire crystals (diameter $18 \mathrm{~mm}$, height $=10 \mathrm{~mm}$, UV grade, Roditi, Union Carbide) produced by the Czochralski method. This material was used as it is transparent in the wave-number range 2000 to $50000 \mathrm{~cm}^{-1}$. The optical transparency at the pulse laser wavelength is not affected by laser irradiation or by changing the temperature.

The optical high-pressure cell is mounted on a metal holder with wooden grip for easy handling and fitting into the sample chamber of the FT-IR spectrometer (see section 4.1.5). 


\subsubsection{OPTICAL INTERNAL CELL}

The optical internal cell used in the kinetic investigations is depicted in Fig. 4.2. The cell consists of a teflon tube (1) (outer diameter $10 \mathrm{~mm}$, inner diameter $9 \mathrm{~mm}$, length $\sim 12 \mathrm{~mm}$ ) which is closed at each end by a calcium fluoride window (2) (diameter $10 \mathrm{~mm}$, thickness $5 \mathrm{~mm}$, Korth). To facilitate optimal sealing of the cylindrical cell, both planar surfaces of the $\mathrm{CaF}_{2}$ windows were polished using a diamond micrometer suspension (4-8 micron, Mikrodiamant $\mathrm{GmbH}$ ). $\mathrm{CaF}_{2}$ was chosen as the window material because it is transparent in the wavenumber range 1100 to $70000 \mathrm{~cm}^{-1}$, thus allowing detection in the desired IR region. The sample volume (3) is contained between the two windows. The internal cell is fitted between the high-pressure windows of the high-pressure cell and held in place by a solid teflon spacer.

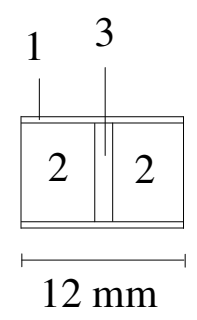

Fig. 4.2 Optical internal cell used for kinetic investigations. (1) teflon tube; (2) $\mathrm{CaF}_{2}$ window; (3) sample volume.

An excellent survey on the high-pressure techniques described within this section can be found in ref. [2].

\subsubsection{Heating AND TeMPERATURe Control}

Heating of the optical high-pressure cell is facilitated by two heating jackets. They consist of a brass matrix, into which is embedded a sheathed resistance heating wire (CGE-Asthom). The closely fitting jackets slide over each end of the cell body (see Fig. 4.1 (3)). The temperature is measured via a sheathed thermocouple (Nickel-chromium against nickel, CIA S250, CGE-Alsthom) and regulated by a PID-controller (Eurotherm 815). 


\subsubsection{Pressure Generation and ConTrol}

For the kinetic investigations, $n$-heptane served as the pressure transmitting medium. The system is pressurized using a manually driven ("syringe"-type) pressure generator (volume $12 \mathrm{~cm}^{3}$ ). Pressure is measured using a high-pressure precision manometer (Class 0.1 , 0-4 kbar, Wiegand). The pressure generating system is depicted in Fig. 4.3.

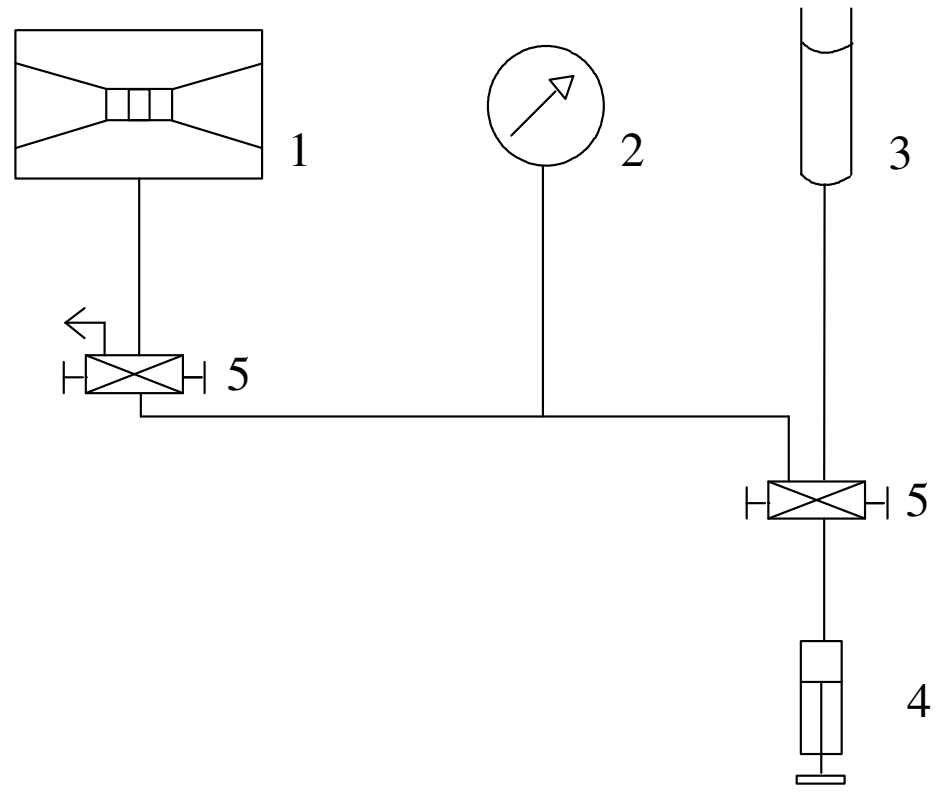

Fig. 4.3 Pressure generating system. (1) optical high pressure cell; (2) manometer; (3) pressure medium $n$-hexane; (4) "syringe"-type pressure generator; (5) valve.

\subsubsection{FT-IR/NIR SPECTROMETER}

Infrared and near infrared spectra were recorded on a Bruker IFS-88 Fourier-Transform Spectrometer. To accommodate the heated optical high-pressure cell, the sample chamber of the spectrometer was enlarged (heightened) and fitted into a water-cooled cell holder (to prevent heat transfer). The chamber is purged with compressed air free of water and carbon dioxide.

For the present work the optical configuration consisted of a halogen source, a silicon-coated calcium fluoride beam splitter, and an InSb detector. This configuration allows for optimal recording in the spectral range 4000 to $10000 \mathrm{~cm}^{-1}$.

Data acquisition and data processing were performed using the Opus software. 


\subsection{THE PLP-SEC TECHNIQUE}

Pulsed laser polymerizations were carried out at 22 to $40{ }^{\circ} \mathrm{C}$ and 1000 bar using an optical high-pressure cell as described in section 4.1.1. Monomer (or comonomer mixture) and photoinitiator (DMPA, $c_{\mathrm{I}}^{0} \approx 5 \cdot 10^{-3} \mathrm{~mol} \cdot \mathrm{L}^{-1}$ ) were mixed and the solution was filled into an internal cell. The internal cell, consisting of a teflon ${ }^{\circledR}$ tube with two $\mathrm{CaF}_{2}$ windows is then inserted into the optical high-pressure cell and the assembly is brought to reaction conditions. The XeF line (351 nm) of an LPX 200 excimer laser (Lambda Physik) was used to induce photoinitiator decomposition and thus copolymerization. The laser repetition rate was selected according to the experimental conditions and to the type of monomer mixture under investigation. The highest repetition rate was $100 \mathrm{~Hz}$. The laser energy per single pulse was close to $8 \mathrm{~mJ}$. Final conversions of about 2 to 4 per cent, but ranging from 1 to 7 per cent in a few cases, were quantitatively measured via near infrared spectroscopic analysis (using a Bruker IFS 88 instrument) of the sample before and after applying a sequence of laser pulses. The polymer was precipitated by adding methanol with approximately $20 \mathrm{ppm}$ hydroquinone immediately after pulsing.

\subsubsection{Size-EXCluSion Chromatography (SEC)}

The SEC setup consists of a Waters 515 HPLC pump, a Rheodyne 7725i injector, and a Waters 2410 differential refractometer. The system has one pre-column and three separation columns: (1) PSS SDV, $8 \times 50 \mathrm{~mm}, 5 \mu$, (2) PSS SDV, $8 \times 300 \mathrm{~mm}, 5 \mu, 10^{5} \AA$, (3) PSS SDV, $8 \times 300 \mathrm{~mm}, 5 \mu, 10^{3} \AA$, (4) PSS SDV, $8 \times 300 \mathrm{~mm}, 5 \mu, 10^{2} \AA$.

Molecular weight distributions (MWDs) were determined with tetrahydrofuran as the eluent at $35^{\circ} \mathrm{C}$ and a flow rate of $1 \mathrm{~mL} / \mathrm{min}$. Data acquisition and processing were carried out using the WinGPC software (PSS, Mainz). The SEC setup was calibrated via narrow molecular weight (MW) polystyrene standards (PSS; Mainz) with MWs ranging from 400 to $2.18 \times 10^{6}$. The copolymer MWDs were obtained via universal calibration with Mark-Houwink parameters determined in this work and partly taken from the literature [3]. 


\subsection{Determination Of Mark-Houwink Parameters}

The samples obtained via PLP-SEC were analyzed according to the principals of universal calibration (section 3.2.2.1), which comprises calibration of the SEC setup using polystyrene (PS) standards and transformation of the molecular weight distribution to the absolute molecular weights (note that this assumes the validity of the Mark-Houwink relation, which may not hold for lower molecular weights). For this transformation, two sets of MarkHouwink parameters are required: for the PS standards and for the polymer of interest (MarkHouwink parameters for PS are $a=0.716$ and $K=1.14 \cdot 10^{-2} \mathrm{~mL} \cdot \mathrm{g}^{-1}$ ). For determination of Mark-Houwink parameters for the polymer of interest, low conversion copolymer samples have been prepared at different initial monomer feed ratios. Approximately $20 \mathrm{~g}$ of monomer mixture are dissolved in $30 \mathrm{ml}$ of toluene and $200 \mathrm{mg}$ AIBN are added. The reaction mixture is degassed for $10 \mathrm{~min}$ by purging with nitrogen and is heated to $60{ }^{\circ} \mathrm{C}$. The onset of copolymerization is monitored by taking samples from the reaction mixture in evenly spaced time intervals of $2 \mathrm{~min}$. These samples are poured into an excess of methanol. Once copolymer precipitates, the reaction mixture is quenched by adding a large excess of methanol. The copolymer is purified by repeated cycles of precipitating and redissolving in dichloromethane. Finally, the copolymer samples are dried at room temperature for at least $24 \mathrm{~h}$ and are subjected to ${ }^{1} \mathrm{H}-\mathrm{NMR}$ analysis for determination of copolymer composition. Mark-Houwink parameters based on analyses of these samples were determined by PSS (Mainz).

\section{4 ${ }^{1} \mathrm{H}-\mathrm{NMR}$}

The procedure of preparing ${ }^{1} \mathrm{H}-\mathrm{NMR}$ samples at low conversions is analogous to the preparation of PLP-SEC samples. The dried copolymer samples are dissolved in dioxane- $\mathrm{d}_{8}$ for MA/MMA or in $\mathrm{CDCl}_{3}$ for all other copolymer systems and measured on a Varian Unity 300 NMR spectrometer at room temperature, a pulse angle of $45^{\circ}$, without relaxation delay and an acquisition time of $2.655 \mathrm{~s}$. 


\subsection{SP-PLP-ESR}

The experimental setup for performing free-radical polymerizations via single pulse-pulsed laser polymerization-electron spin resonance technique (SP-PLP-ESR) is given in Fig. 4.4.

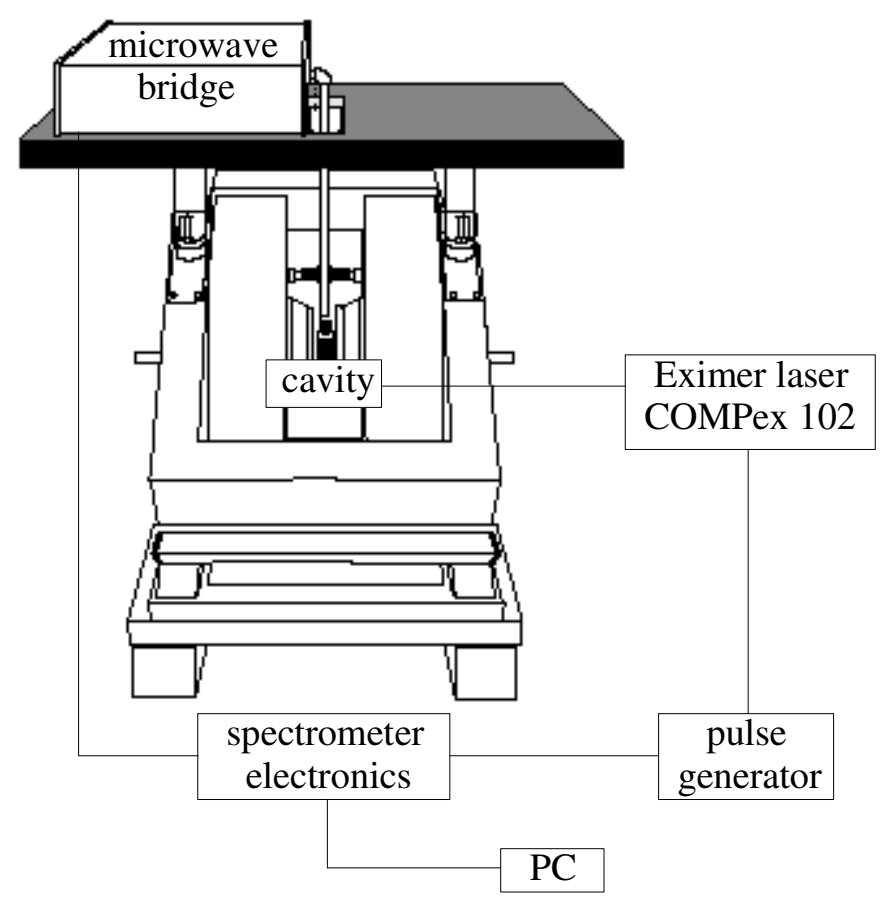

Fig. 4.4 Experimental setup for the single pulse-pulsed laser polymerization-electron spin resonance (SP-PLP-ESR) technique.

ESR spectra were recorded on a Bruker Elexsys ${ }^{\circledR}$ E 500 series CW-EPR spectrometer operating in the X-band at a $100 \mathrm{kHz}$ modulation field and a microwave power of $10 \mathrm{~mW}$. The photoinitiator MMMP was used at concentrations of about $2 \cdot 10^{-2}$ to $5 \cdot 10^{-3} \mathrm{~mol} \cdot \mathrm{L}^{-1}$. In a glove box, under an argon atmosphere, MMMP was added to the monomer and the solution was filled into a $5 \mathrm{~mm}$ outer diameter ( $4 \mathrm{~mm}$ inner diameter) quartz tube. The tube was placed into a cavity and irradiated through a grid by a COMPex 102 excimer laser (Lambda Physik) working on the $\mathrm{XeF}$ line $(351 \mathrm{~nm})$ positioned at a distance of about $50 \mathrm{~cm}$ from the tube. The laser energy was around $50 \mathrm{~mJ}$ per pulse. In all cases, the laser beam passed through the sample tube at right angle to the main axis of the tube. The spectrometer and the laser were triggered using a Scientific Instruments 9314 pulse generator. After applying a single laser pulse, the decay of radical concentration was measured as follows: As the average life-time of a growing radical chain is extremely short, there is insufficient time to scan the entire ESR spectrum. In order to achieve the optimum signal-to-noise ratio the absolute maximum of the 
spectrum (indicated by the arrow in Fig. 4.5) was chosen. The decay in intensity at this field position was then recorded [4]. In most cases, several experimental traces were co-added to improve the signal-to-noise ratio (Fig. 4.6).

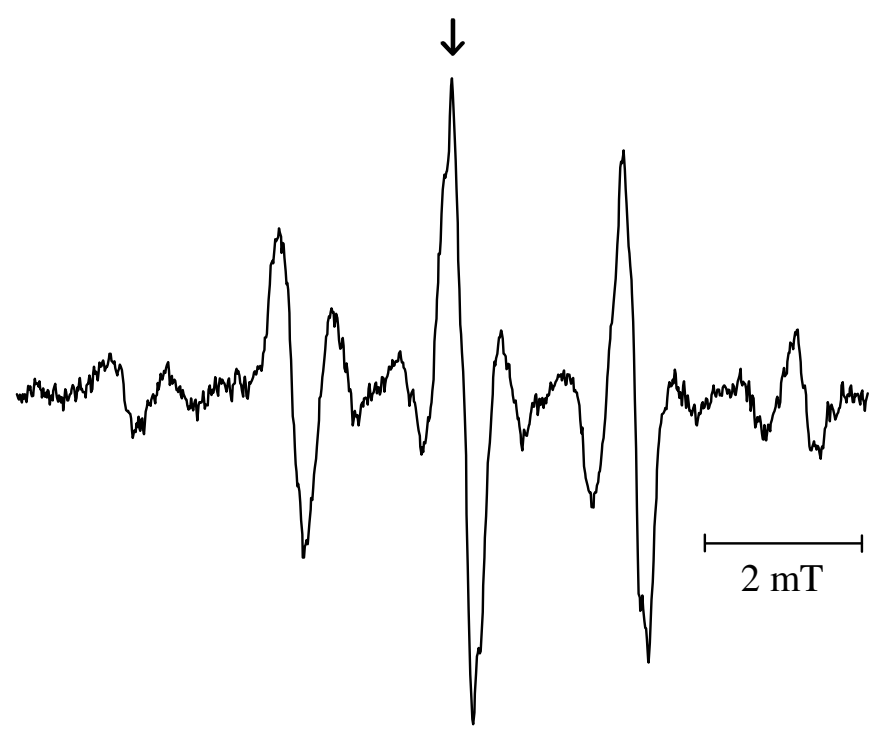

Fig. 4.5 ESR spectrum of DMA macroradicals from quasi-stationary polymerization initiated by laser pulses applied at a repetition rate of $20 \mathrm{~Hz}$ at $0{ }^{\circ} \mathrm{C}$ (time constant $10.24 \mathrm{~ms}$, sweep time $10.49 \mathrm{sec}$ ) with the MMMP photoinitiator concentration $c_{\text {MMMP }}=25.5 \cdot 10^{-3} \mathrm{~mol} \cdot \mathrm{L}^{-1}$. 


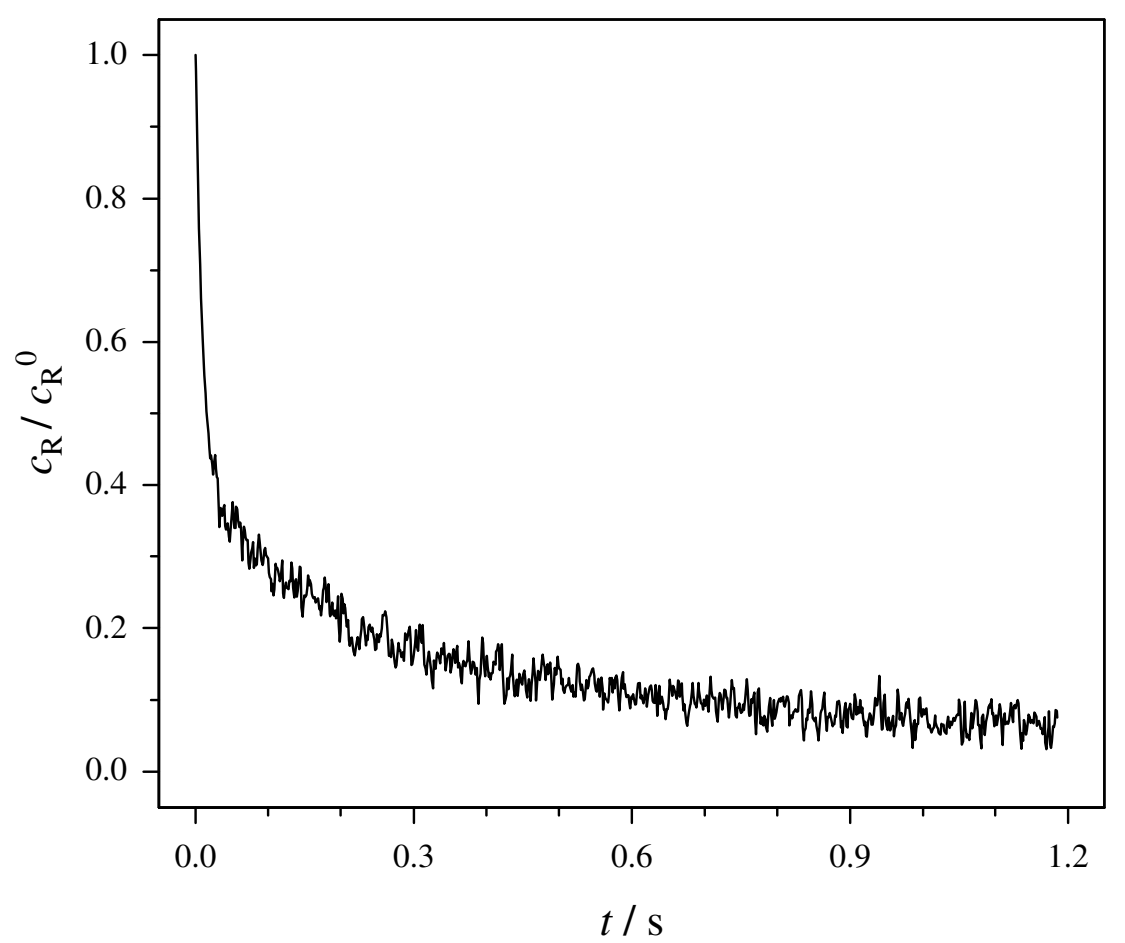

Fig. 4.6 Decay in radical concentration after applying a laser pulse at $t=0$ as measured by ESR for a DMA bulk polymerization at $0{ }^{\circ} \mathrm{C}$ and a content of $14 \%$ polyDMA from preceding DMA photo-polymerization.

In order to determine the radical concentration as a function of time, a twofold calibration is required. The usual method for measuring the concentration of free radicals in a sample with an unknown concentration of radicals is to compare its ESR signal with that of a sample containing a known quantity of free radicals. From theory it is known that the number of spins is proportional to the area under the absorption curve. The derivative signal has to be integrated to obtain this area [5]. By using the stable free-radical TEMPO $(2,2,6,6-$ tetramethyl-1-piperidinyloxyl), the correlation between the double integral of a ESR-spectrum and absolute radical concentration is first deduced for typically experimental conditions. ESR spectra of TEMPO were measured under conditions of temperature, pressure, solvent, sample volume, etc. as close as possible to that of the actual SP-PLP-ESR experiment.

Recording a full ESR spectrum, as the one depicted in Fig. 4.5, which is associated with the variation of magnetic field, takes a few seconds. To measure radical concentration with millisecond time resolution, the ESR signal was monitored at a fixed magnetic field. To achieve good signal-to-noise quality, the magnetic field position associated with the peak maximum (arrow in Fig. 4.5) was selected for this time-resolved analysis. The quantitative measurements rest on the proportionality between radical concentration and the double integral of the associated ESR spectrum [6], which correlation holds irrespective of the type of free radical under investigation. That there is a linear correlation between the intensity at 
the peak maximum and the double integral of an ESR spectrum was carefully checked by recording several complete spectra under quasi-steady-state initiation conditions before carrying out each experiment. Different macroradical concentrations were produced by varying laser energy in quasi-stationary polymerizations carried out at pulse repetition rates of $20 \mathrm{~Hz}$. Under typical conditions this correlation was indeed very close to linear (Fig. 4.7).

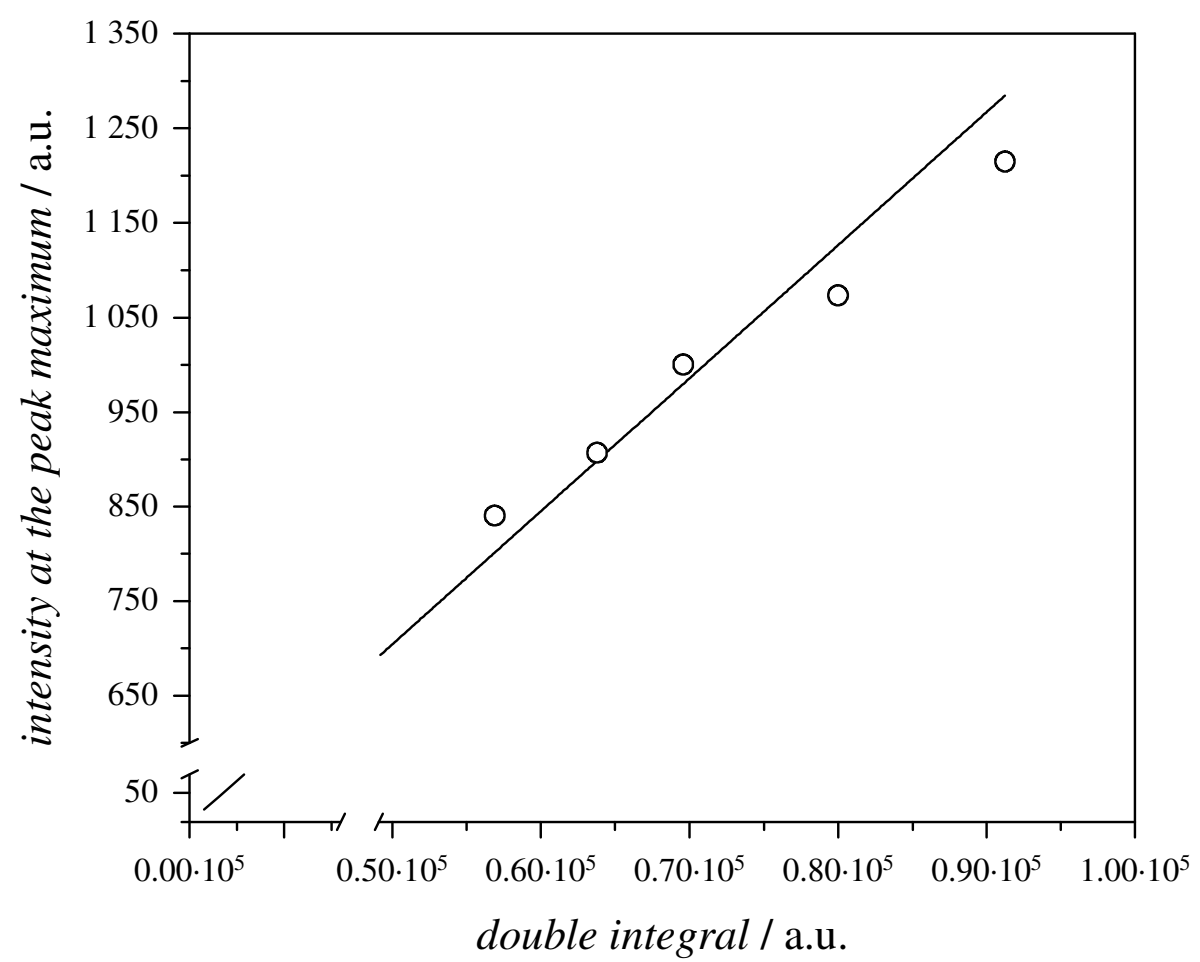

Fig. 4.7 Correlation between the double integral of the ESR spectrum of DMA and the intensity at the peak maximum, obtained from quasi-stationary polymerization initiated by laser pulses applied at a repetition rate of $20 \mathrm{~Hz}$ at $0{ }^{\circ} \mathrm{C}$ with the MMMP photoinitiator concentration $c_{\mathrm{MMMP}}=25.5 \cdot 10^{-3} \mathrm{~mol} \cdot \mathrm{L}^{-1}$.

In between the PLP experiments, the ampoule was removed several times from the ESR spectrometer and inserted into the sample chamber of an IFS 88 FT-NIR spectrometer (Bruker) to measure absolute (overall) monomer concentration. The monomer conversion was determined by monitoring the absorbance of the first overtone of the stretching vibration of the $\mathrm{C}-\mathrm{H}$ mode (at the $\mathrm{C}=\mathrm{C}$ double bond) at around $6160 \mathrm{~cm}^{-1}$ [7].

For data acquisition, the software Xepr v1.0 (provided by Bruker) which, in addition, controls all spectrometer settings, was used. For data analysis, e.g. for modeling termination rate coefficients, the software packages Matlab ${ }^{\circledR}$ and Origin ${ }^{\circledR}$ were used. 
ESR investigations on highly polar media, such as some pure methacrylates or acrylates pose problems as, e.g. the resonance frequency is not easily identified. It is for this reason that some polymerizations have been carried out in solution of toluene.

\subsection{THE SP-PLP-NIR TECHNIQUE}

\subsubsection{Details ABOUT THE SETUP AND ElECTRONICS}

The principal experimental setup for the SP-PLP experiments of this work is illustrated in Fig. 4.8 [see also 8,9]. For initiation of the photopolymerization, XeF excimer laser pulses at $351 \mathrm{~nm}$ (Lextra 50, Lambda Physics) are reflected on the optical axis of the sample cell by UV mirrors (S) transparent in the infra-red region (INFRASIL, ZnSe-coated). A detector (D), which is calibrated before each measurement [8], is used to determine the intensity of the laser radiation in front of the optical high pressure cell. A photo-shutter FV (Prontor, magnetic-shutter E/40) can be used to select laser pulses.

A tungsten-halogen lamp La (General electric, $75 \mathrm{~W}$ ) serves as a source of infrared and near infrared radiation. The lamp is powered by two lead accumulators (12V, $220 \mathrm{~A} \cdot \mathrm{h})$ to achieve a noise-free signal. A second photo-shutter (FV), directly in front of the monochromator, is used to block out the analysis light to measure the detector signal without near infrared radiation. Pre-initiation processes by UV parts in the spectrum of the tungsten-halogen lamp are suppressed by a UV cut-off filter F1 (RG 695). The analysis light is focused by a lens L1 $\left(\mathrm{CaF}_{2}, \mathrm{f}=100 \mathrm{~mm}, \mathrm{~d}=50 \mathrm{~mm}\right)$ to the sample contained in the optical high-pressure cell. A second lens $\mathrm{L} 2\left(\mathrm{CaF}_{2}, \mathrm{f}=100 \mathrm{~mm}, \mathrm{~d}=50 \mathrm{~mm}\right)$ focuses the analysis light onto the lid of a monochromator (B\&M Spectronik, BM 50, 0.5, f/6.9). The light is then diffracted on an interference grating (Bausch\&Lomb, $76 \mathrm{~mm} \cdot 76 \mathrm{~mm}$ ), 600 1/mm, blaze $1.6 \mathrm{~mm}$, $\mathrm{D}=4.1 \mathrm{~nm} / \mathrm{mm}$ ) and reflected to a fast NIR detector by an ellipsoidal mirror $\mathrm{E}$ (Bruker Analytische Meßtechnik GmbH, f/6, $\left.\mathrm{f}_{1}=200 \mathrm{~mm}, \mathrm{f}_{2}=40 \mathrm{~mm}\right)$. 


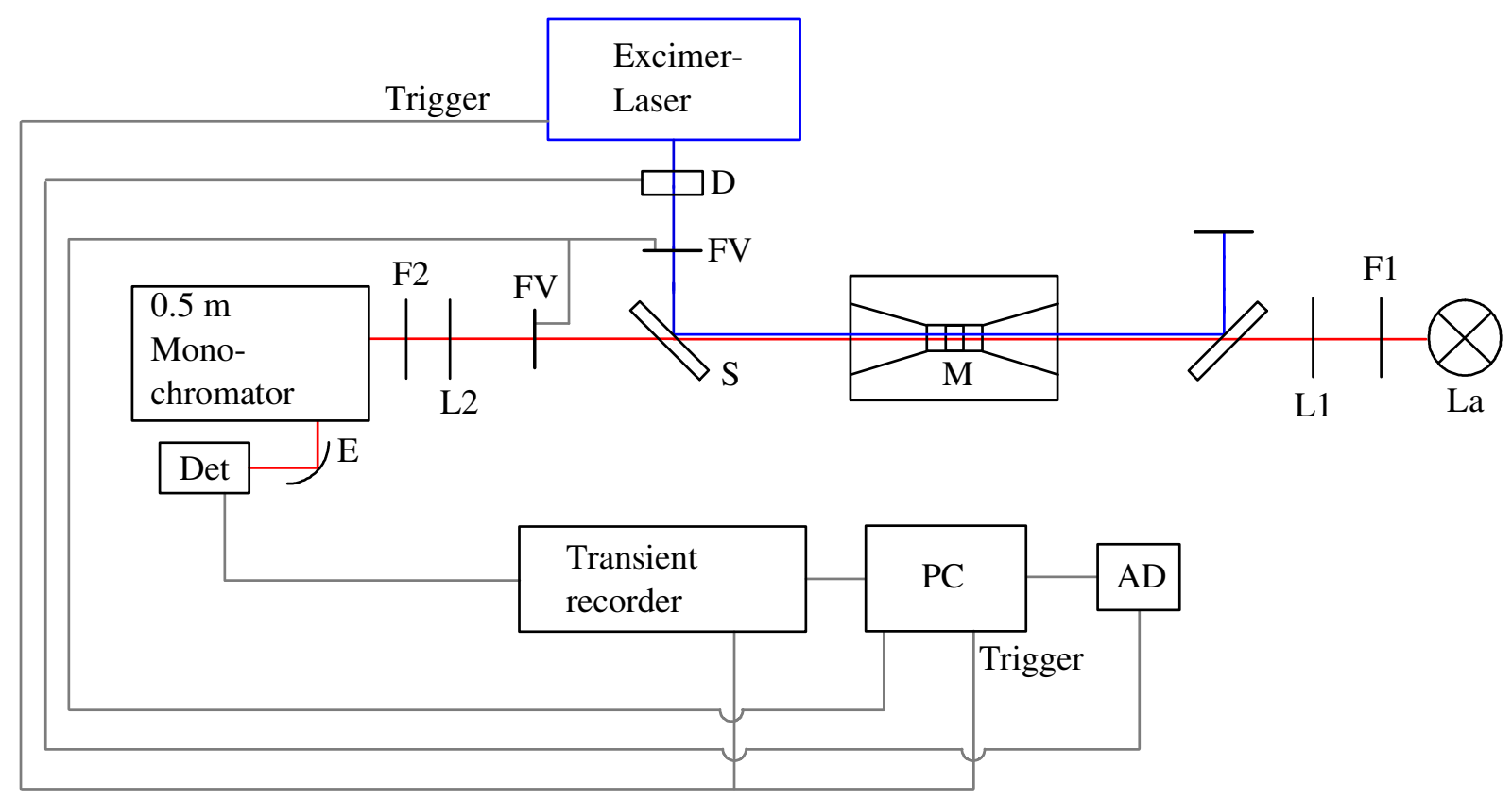

Fig. 4.8 Experimental setup for the single pulse-pulsed laser polymerization (SP-PLP) technique.

A silicium filter F2 (Oriel, $1 \mathrm{~mm}, 1.05 \mu \mathrm{m}$, transmission in the 5000 to $9000 \mathrm{~cm}^{-1}$ range) ensures that only one grating order hits the detector. The analog detector signal is recorded by a 16-bit transient recorder (TR 1621-4, Fast ComTech) and transferred to a computer for further evaluation. To reduce potential vibrations of the building, the entire setup is placed on a solid granite board, which is supported by rubber tubes [10].

\subsection{FT-NIR SPECTROSCOPY}

Monomer conversion was controlled by FT-NIR spectroscopy. It has become standard practice $[11,12,13]$ to use the first overtone of $\mathrm{CH}$-vibrations with the hydrogen atom being linked to a $\mathrm{C}=\mathrm{C}$ double bond for quantitative analysis of olefinic monomers. This absorbance is located at around $6160 \mathrm{~cm}^{-1}$ for acrylates and methacrylates, which is well suited for analysis of monomer conversion, because it is not overlapped by other bands, and because its absorbance is proportional to monomer concentration. To deduce the overall monomer conversion from the spectra, Beer-Lambert's law needs to be valid. It relates the absorbance $A$ (at a specific wave number, $\tilde{v}$ ) with monomer concentration, $c_{\mathrm{M}}$ :

$$
A(\widetilde{v})=\log \left(I / I^{0}\right)=\varepsilon(\widetilde{v}) \cdot c_{\mathrm{M}} \cdot l
$$

In Eq. $4.1, \varepsilon$ is the molar absorption coefficient, $l$ is the optical path length, $I^{0}$ is light intensity going to the sample cell, $I$ is the light intensity coming from the sample cell. 
The peak is integrated over the high-wavenumber half-band from the band maximum toward higher wavenumbers against a horizontal baseline through the absorbance at $6300 \mathrm{~cm}^{-1}$. Monomer conversion $X$ is obtained from the ratio of integrals after starting polymerization Int $_{\mathrm{p}}$ and the integral at time $t=0$ :

$$
\operatorname{Int}_{0}: X=\left[1-\left(\operatorname{Int}_{\mathrm{p}} / \operatorname{Int}_{0}\right)\right] \cdot 100
$$

where

$$
\operatorname{Int}_{0}=\int_{\tilde{v}_{1}}^{\tilde{v}_{2}} A(\tilde{v}) \mathrm{d} \tilde{v}=B \cdot c_{\mathrm{M}} \cdot l
$$

( $B$ is integrated molar absorptivity)

Fig. 4.9 shows typical NIR spectra of DA between $6350 \mathrm{~cm}^{-1}$ and $6050 \mathrm{~cm}^{-1}$ at $60{ }^{\circ} \mathrm{C}$ and 1 bar. The insert shows an absorbance spectrum between $6500 \mathrm{~cm}^{-1}$ and $5000 \mathrm{~cm}^{-1}$.

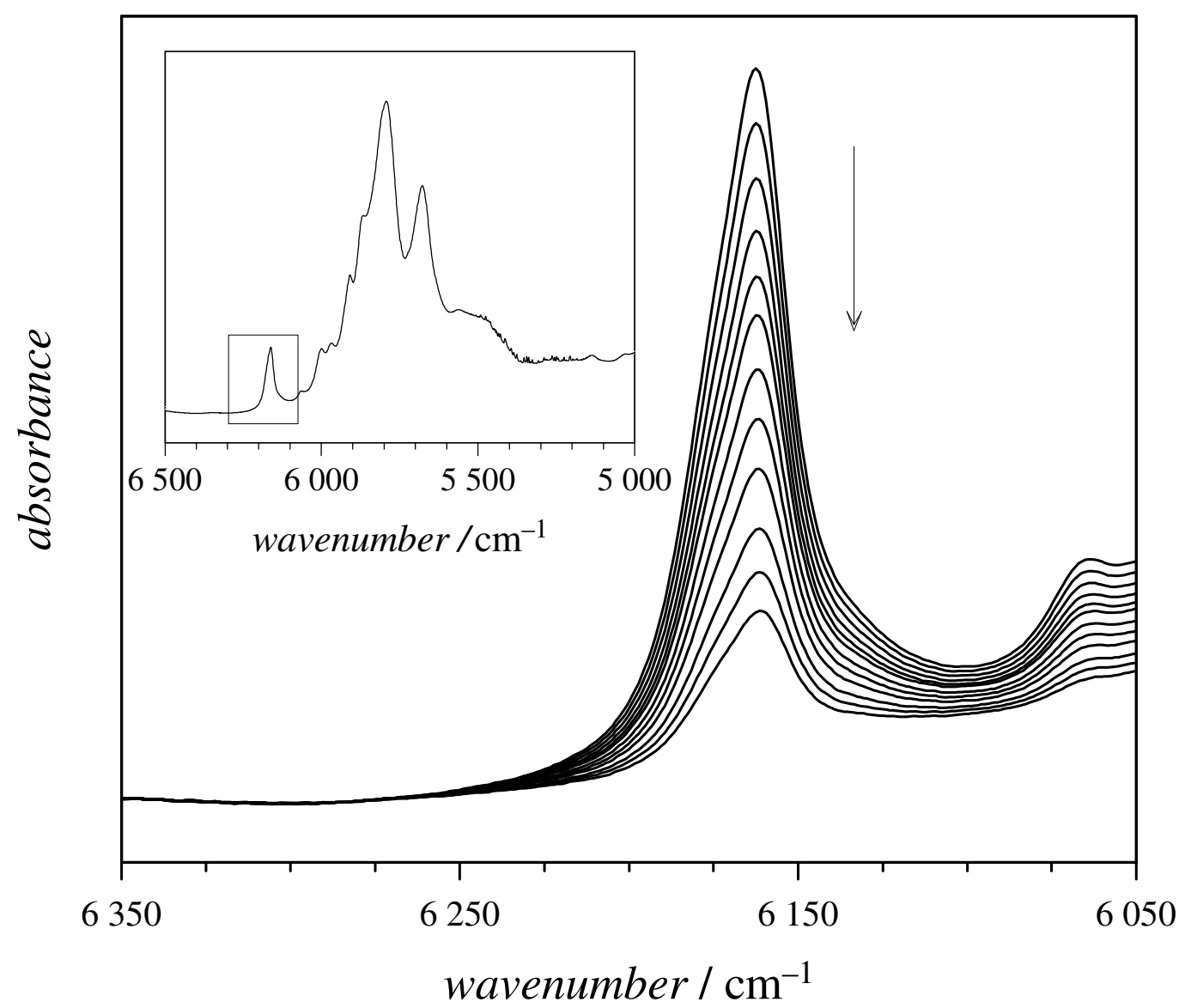

Fig. 4.9 NIR spectra recorded during a chemically initiated DA polymerization at $60{ }^{\circ} \mathrm{C}$ and 1 bar. 


\subsection{Simulation ToOl PREDICI ${ }^{\circledR}$}

The kinetic models described in this work are implemented into the simulation program PREDICI $^{\circledR}$ (Polyreaction Distributions by Countable System Integration) developed by M. Wulkow. [14,15] The program uses a highly efficient algorithm, called discrete hpalgorithm for solving complex sets of countable differential equations. An integrated function interpreter enables the coupling of the rate coefficients of elementary reaction steps with individual species concentrations, conversions, copolymer composition, number and weight average of the formed polymer. As a special feature of PREDICI ${ }^{\circledR}$, the full MWD can be derived without any assumptions of closure conditions. 


\subsection{SUBSTANCES USED}

\section{MONOMERS}

The acrylates $\left(\mathrm{R}_{2}=\mathrm{H}\right)$ and methacrylates $\left(\mathrm{R}_{2}=\right.$ Methyl $)$ have the following general structure:

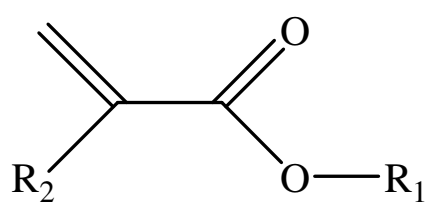

Methyl Acrylate and Methyl Methacrylate

Methyl acrylate (MA) (Fluka, purum 99.5\%) and methyl methacrylate (MMA) (Fluka, $99 \%$, stabilized by $0.02 \%$ hydroquinone monomethyl ether) were distilled (using a Vigreux column) under reduced pressure $\left(\approx 47^{\circ} \mathrm{C}, 240\right.$ Torr $)$ in the presence of $\mathrm{K}_{2} \mathrm{CO}_{3}$ to remove the stabilizer. Great care has to be taken not to contaminate the monomer by vacuum grease. Therefore the distillation apparatus is kept sealed by teflon washers.

Butyl Acrylate

Butyl acrylate (BA) (Fluka, purum $>99 \%$, stabilized by $0.02 \%$ hydroquinone monomethyl lether) was distilled under reduced pressure $\left(\approx 50{ }^{\circ} \mathrm{C}, 50\right.$ Torr $)$ in the presence of $\mathrm{K}_{2} \mathrm{CO}_{3}$ to remove the inhibitor.

\section{Dodecyl Acrylate and Dodecyl Methacrylate}

Dodecyl methacrylate (DMA) (Fluka, $95 \%$ ) and dodecyl acrylate (DA) (Fluka, techn.), which is actually a mixture of dodecyl acrylate $(55 \%)$ and tetradecyl acrylate $(45 \%)$, were distilled under reduced pressure in the presence of $\mathrm{K}_{2} \mathrm{CO}_{3}$ to remove the inhibitor hydroquinone monomethyl ether. DA was further purified via column chromatography with silicagel in solution of $n$-pentane (90 vol \%) and $d i$-ethylether (10 vol \%). DMA was filtrated using an inhibitor remover (Aldrich). 
Cyclohexyl Methacrylate

Cyclohexyl methacrylate (CHMA) (Fluka, purum $97 \%$, stabilized by $0.006 \%$ hydroquinone monomethyl ether) was distilled under reduced pressure in the presence of $\mathrm{K}_{2} \mathrm{CO}_{3}$ to remove the inhibitor.

\section{Benzyl Methacrylate}

Benzyl methacrylate (BzMA) (Aldrich, purum $96 \%$, stabilized by 50 ppm hydroquinone monomethyl ether) was distilled under reduced pressure $\left(\approx 130^{\circ} \mathrm{C}, 40\right.$ Torr $)$ in the presence of $\mathrm{K}_{2} \mathrm{CO}_{3}$ to remove the inhibitor. Additionally BzMA was filtrated using an inhibitor remover (Aldrich).

\section{Dodecyl Acrylate Dimer}

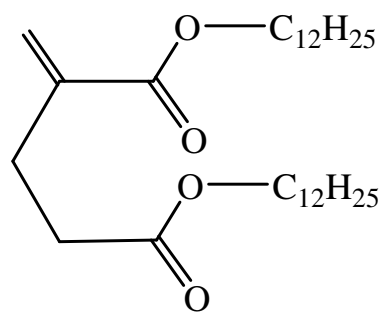

Dodecyl acrylate dimer (DAD) was synthesized according to the procedure suggested by Trumbo et al. [16]. To a $500 \mathrm{~mL}$ round bottom flask equipped with a magnetic stirrer, reflux condenser, thermometer, addition funnel, and nitrogen inlet were charged $60 \mathrm{~mL}$ of THF and $10 \mathrm{~mL}$ of tri- $n$-butyl phosphine $\left(n-\mathrm{Bu}_{3} \mathrm{P}\right)$. This solution was cooled to $10{ }^{\circ} \mathrm{C}$ by means of an ice bath and dodecyl acrylate $(100 \mathrm{~mL})$ was added dropwise from the additional funnel over a period of $2 \mathrm{~h}$ to the vigorously stirred solution. After addition of all DA, the reaction mixture was stirred at $45^{\circ} \mathrm{C}$ for $3 \mathrm{~h}$ and than at room temperature overnight. The dimer was isolated by column chromatography on silicagel with a solution of $n$-pentane $(90$ vol $\%)$ and $d i$ ethylether $\left(10\right.$ vol \%). The yield was $60 \%$. The structure of DAD was proved by ${ }^{1} \mathrm{H}-\mathrm{NMR}$.

\section{SOLVENTS}

Toluene (Fluka, 99.5\%), methanol (Fluka, p.a.) and dichloromethane (Aldrich, p.a.) were used without further purification. For the SEC analysis THF (Roth, > 99.5 \%, p.a.) was used. 


\section{INITIATORS, INHIBITORS AND STABLE RADICALS}

2,2-Dimethoxy-2-phenylacetophenone (DMPA)<smiles>COC(OC)(C(=O)c1ccccc1)c1ccccc1</smiles>

The photoinitiator DMPA (Aldrich, $99 \%$ ) was used as received.

2-Methyl-4-(methylthio)-2-morpholino-propiophenone (MMMP)

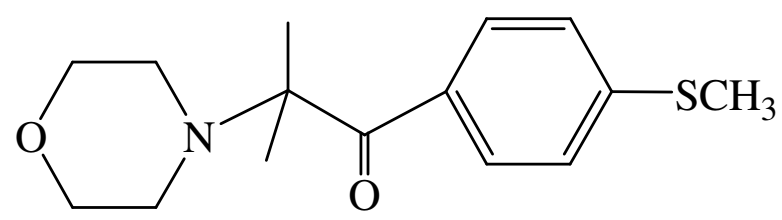

The photoinitiator MMMP (Aldrich, > $98 \%$ ) was used without further purification.

Azo-bis-isobutyronitrile (AIBN)

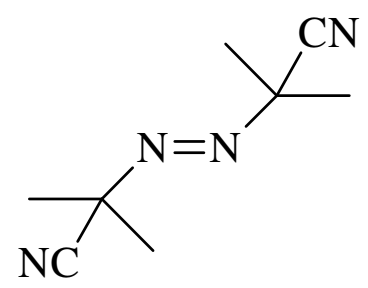

AIBN (Aldrich, 99.5\%) was recrystallized twice from diisopropylether prior to use.

tert-Butylperoxy-2-ethylhexanoate (TBPO)<smiles>CCCCC(CC)C(=O)OOC(C)(C)C</smiles>

For chemically initiated polymerization TBPO (AKZO NOBEL, $98.7 \%$ ) was used without further purification. 


\section{Hydroquinone}

Hydroquinone (Aldrich, $>99 \%$ ) was used as received.

2,2,6,6-tetramethyl-1-piperidinyloxyl (TEMPO)

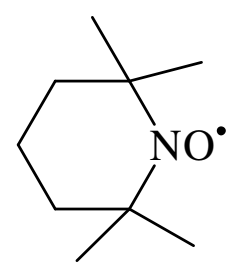

2,2,6,6-tetramethyl-1-piperidinyloxyl (TEMPO) (Aldrich, 99\%) was used as received. 


\subsection{ON THE SELECTION OF EXPERIMENTAl CONDitions}

\subsubsection{LASER-InduCEd Polymerization In PLP-SEC EXPERIMENTS}

Values of $k_{\mathrm{p}, \text { copo }}$ in acrylate/methacrylate systems were measured at the temperatures between 22 and $40{ }^{\circ} \mathrm{C}$. All experiments were carried out at 1000 bar. The propagation rate coefficients of acrylates have been known to depend on the PLP conditions [17,18]. Particular difficulties arise at temperatures above $30{ }^{\circ} \mathrm{C}$. Therefore temperatures close to $22{ }^{\circ} \mathrm{C}$ were chosen. Data at $40{ }^{\circ} \mathrm{C}$ were taken to provide the additional points at the high acrylate contents and thus to extend the already measured $k_{\mathrm{p}, \mathrm{copo}}$ data for the systems DA/DMA and MA/DMA [3].

\subsubsection{A TYPICAL SP-PLP-NIR EXPERIMENT}

The optical high-pressure cell, which at this point only contains an empty internal cell with the $\mathrm{CaF}_{2}$ windows in contact, is brought to the reaction temperature and a NIR reference spectrum is recorded. The optical cell is then pressurized and the UV energy detector is calibrated against a joulemeter (Gentec).

The monomer is purified and degassed by several freeze-pump and thaw cycles, the photoinitiator is added under an argon atmosphere in a glove box. The process of assembling the internal cell is detailed elsewhere [2]. After inserting the internal cell, the optical highpressure cell is brought to reaction conditions. An initial NIR spectrum is recorded and the monochromater is set to the desired wavelength. The initial intensity at the wavelength is measured and data concerning details of the measurement (such as the time between data points, amplifier range, the number of signals that are co-added, etc.) are transferred to the transient recorder. The maximum number of points that can be recorded is 4096 . The transient recorder measures the change in light intensity after firing a laser pulse as a voltage vs. time signal. The data is then transferred to a computer for further evaluation. In regular intervals, NIR spectra over an extended wavenumber range are recorded to deduce overall monomer conversion. 


\subsubsection{LASER-INDUCED POLYMERIZATION IN SP-PLP-ESR}

ESR sensitivity (net absorption) increases with decreasing temperature. Taking this into account, one obtains better quality of a signal at lower temperature. On the other hand, relatively high temperatures are required for comparing with data from other techniques. For these reasons a temperature interval between $-50{ }^{\circ} \mathrm{C}$ and $70^{\circ} \mathrm{C}$ was chosen for the experiments.

There are, of course, some difficulties in applying the time-resolved ESR to pulsed laser polymerization. Most important is the problem of detecting rapid changes in radical concentration of $10^{-5}$ to $10^{-8} \mathrm{~mol} \cdot \mathrm{L}^{-1}$ on a short (milliseconds or better) time scale, which makes the use of this technique limited to monomers with relatively low $k_{\mathrm{t}}$ (and $k_{\mathrm{p}}$ ). Another difficulty is the uncertainty associated with calibration of absolute radical concentrations, which is the intrinsic problem of all ESR-based methods.

In case of SP-PLP it was shown that too high as too low initiator concentration have a negative influence on $k_{\mathrm{t}}[19,20]$. In this work an initiator concentration of $3 \cdot 10^{-2}$ $5 \cdot 10^{-3} \mathrm{~mol} \cdot \mathrm{L}^{-1}$ was chosen. As initiator for SP-PLP-ESR measurements was taken MMMP.

\subsubsection{ChEMiCAlly Initiated Polymerization}

In case of chemically initiated polymerizations, the reaction was induced by thermal decomposition of TBPO. The experiments were conducted at ambient pressure and temperatures between 60 and $80{ }^{\circ} \mathrm{C}$. For the polymerization the inner cell is inserted into the heated high-pressure cell in order to shorten the heating-up phase. The lower limit for the temperature was obtained from the decomposition constant of TBPO, the upper limit from the polymerization rate, because at higher temperatures the time required to assemble the highpressure cell cannot longer be neglected relative to the reaction time. Monomer conversion during the polymerization is controlled via NIR.

The use of TBPO as the initiator of the chemically initiated polymerization is recommended, because of its high solubility in such monomers as DA and DMA. The values of the initiator efficiency, $f$, determined by Sandmann in ethene polymerization and the rate coefficient of TBPO decomposition, $k_{\mathrm{d}}$, determined in $n$-heptane as a function of temperature and pressure were used [21]. These numbers were adopted for the polymerizations of the present study. The TBPO concentrations used in this study were between 0.02 and $0.20 \mathrm{~mol} \cdot \mathrm{L}^{-1}$. 


\subsection{ERROR ESTIMATE}

Measurement of the reaction temperature in the direct vicinity of the optical path of the highpressure cell is achieved with a sheathed thermocouple to better $\pm 0.3 \mathrm{~K}$. The PID controller regulates the temperature within $\pm 0.2 \mathrm{~K}$. An accuracy of $\pm 0.5 \mathrm{~K}$ is therefore gained for the temperature measurement.

The high-pressure precision manometer of class 0.1 offers an accuracy of $\pm 0.1 \%$ of the maximum pressure, resulting in an error of \pm 4 bar.

For the preparation of the comonomer solutions, an analysis balance (Sartorius) was used which allows measurements up to 4 decimal places. The absolute error should therefore be $\pm 0.05 \mathrm{mg}$. However, the actual error in concentration measurement is greater than this, as the balance is very sensitive to small disturbances. The real error in comonomer solutions is estimated to be below $\pm 2 \%$. The absolute value of the concentration of the solution at reaction conditions is fairly large due to uncertainties in the PVT-data available from the literature [22], but should not exceed $10 \%$. The relative error of concentration measurement is much smaller and is assumed to be below $\pm 3 \%$.

The FT-IR spectrometer used in this work affords quantitative evaluation of absorbances measured in the range of 0.02 to 0.8 with the optical configuration employed. The lower boundary is determined by the signal-to-noise ratio, and the upper limit by non-linearity of the detector. For absorbances in the above-mentioned range, the error due to non-linearity is less than $3 \%$ [10]. Further errors are introduced by the apodisation function (weighting factor in the spectrum calculation) and the mode of phase correction in the Fourier transform step. The uncertainty in determination of the base line results in an error in the integrated absorbance (used for quantitative evaluation) of less than $\pm 3 \%$ for a measured real absorbance of approximately 0.5 . Due to the uncertainty in the position of the maximum, this error is raised to $\pm 5 \%$ in the evaluation of half-band integrals. For any experiment, the relative error in the integrated absorbance results in a maximum error of $\pm 6 \%$.

The error of the PLP-SEC measurements is composed of several errors: The error in the density of the comonomer mixture at reaction conditions and the copolymer composition $\left(F_{1}\right)$, the error induced by the SEC calibration procedure via Mark-Houwink coefficients and the errors induced by SEC broadening. These errors add up to a total of $\pm 30 \%$ for the 
propagation rate coefficients of this work. The errors of the $k_{\mathrm{p}}$ values determined by PLP-SEC for homopolymerizations that do not require indirect calibration (e.g. PMMA or PS) are close to $\pm 15 \%$.

The error in the termination rate coefficient, $k_{\mathrm{t}}$, is considered to be close to $\pm 50 \%$ [10] for most polymerization systems of this work.

The errors in the measurement of the copolymer composition by ${ }^{1} \mathrm{H}-\mathrm{NMR}$ spectroscopy is dependent on the copolymer system under investigation. For systems with a clear separation of the NMR peaks used for integration, the error in copolymer composition is less than $3 \%$. This applies to MA/DMA and DA/MMA copolymerization. In case of a peak overlap the error is close to $\pm 8 \%$.

The errors of parameters which are deduced by a non-linear least squares fitting procedure (including the simple case of linear regression) are determined using statistical methods and are given together with the respective value in the text.

In the SP-PLP-ESR experiments, the ratio of double integral to peak intensity, within a scatter of about \pm 6 per cent, is insensitive toward free-radical concentration and toward detection sensitivity. The determination of radical concentration is assumed to be accurate within \pm 30 per cent. Relative radical concentration, $c_{\mathrm{R}}(t) / c_{\mathrm{R}}{ }^{0}$, where $c_{\mathrm{R}}{ }^{0}$ represents the initial concentration of radical species formed by addition of the first monomer molecule to a primary photoinitiator-derived radical, is measured with higher accuracy, as $c_{\mathrm{R}}(t) / c_{\mathrm{R}}{ }^{0}$ is directly obtained without applying a calibration procedure.

In the ESR experiments the error of an amount of mid-chain radicals in a system at certain conditions is close to $\pm 7 \%$. 


\subsection{REFERENCES}

[1] Poulter, T. C. Phys. Rev. 1932, 40, 860.

[2] Buback, M.; Hinton, C. in High-pressure Techniques in Chemistry and Physics - A practical approach, Holzapfel, B . W and Isaacs, N.S, eds., Oxford University Press: Oxford, 1997.

[3] Buback, M.; Feldermann, A.; Barner-Kowollik, C.; Lacik, I. Macromolecules. 2001, $34,5439$.

[4] Zhu, S.; Tian, Y.; Hamielec, A.E. Macromolecules. 1990, 23, 1144.

[5] Randy, B.; Rabek, j. F. ESR spectroscopy in Polymer research, Springer-Verlag, Berlin, 1977.

[6] Tonge, M. P.; Kajiwara, A.; Kamachi, M.; Gilbert, R. G. Polymer 1998, 39, 2305.

[7] Buback, M. Angew. Chem. 1991, 103, 658.

[8] Schweer, J. Ph. D. Thesis, Göttingen, 1988.

[9] Buback, M.; Hippler, H.; Schweer, J.; Vögele, H. P. Macromol. Chem., Rapid Commun. 1987, 8, 473.

[10] Kowollik, C. Ph. D. Thesis, Göttingen, 1999.

[11] Buback, M. Angew. Chem. Int. Ed. Engl. 1991, 30, 641.

[12] Schmaltz, C. Ph. D. Thesis, Göttingen, 1997.

[13] Buback, M.; Kuchta, F.-D. Macromol. Chem. Phys. 1995, 198, 1455.

[14] Deufelhard, P.; Wulkow, M. Konrad Zuse Zentrum, Berlin, Preprint SC94-22, 1994.

[15] Wulkow, M. Macromol. Theory Simul. 1996, 2, 393.

[16] Trumbo, D. L.; Zander, R. A. J. Of Polymer Sci.: Part A: Polymer Chemistry 1991, 29, 1053.

[17] Beuermann, S.; Paquet, D. A.; McMinn Jr., J. H.; Hutchinson, R. A. Macromolecules, 1996, 29, 4206.

[18] Arzamendi, G.; Plessis, C.; Leiza, J. R.; Asua, J. M. Macromol. Theory Simul. 2003, 12,315 .

[19] Vana, P.; Davis, T. P.; Barner-Kowollik, C. Aust. J. Chem. 2002, 55, 315.

[20] Feldermann, A. Ph. D. Thesis, Göttingen, 2003.

[21] Sandmann, J. Ph. D. Thesis, Göttingen, 2000.

[22] Kurz, C. Ph. D. Thesis, Göttingen, 1995. 


\section{Propagation}

This section focuses on the determination of propagation rate coefficients, $k_{\mathrm{p}}$, for homo- and copolymerizations. The experimental data for different acrylate/methacrylate systems and different temperatures are exclusively from PLP-SEC measurements.

The application of PLP-SEC to acrylates suffers from significant difficulties as has been outlined by van Herk [1] and within a recent IUPAC paper on $n$-butyl acrylate [2]. Above $30{ }^{\circ} \mathrm{C}$, the PLP-generated molecular weight distributions are losing their characteristics and become broad. Suggestions that have been put forward by van Herk [1] to explain this behavior include reaction mechanisms that result in a break-down of the narrow radical chainlength distribution. These mechanisms include transfer to monomer and inter/intra-molecular chain transfer to polymer. However, in order to explain the failure of the PLP-experiment by chain transfer to monomer, unrealistically high transfer coefficients were needed [3]. Chain transfer to polymer, on the other hand, is a known phenomenon and has received considerable attention by the group of Lovell $[4,5,6]$. Especially at low polymer concentrations and low monomer conversions, intramolecular chain transfer to polymer occurs significantly. The occurrence of mid-chain radicals, created by transfer to polymer process, has been confirmed by electron spin resonance (ESR) spectroscopy [7], and by the observation of quaternary carbons with ${ }^{13} \mathrm{C}$-NMR under pulsed laser polymerization conditions [8]. In section 7 it will be shown that even at $30{ }^{\circ} \mathrm{C}$ the majority of all radicals in BA polymerization are mid-chain radicals. This high amount of mid-chain radicals may cause the featureless MWD produced in PLP-SEC experiments, because two types of radicals are involved in chain growth and chains of different length are formed.

For the reasons given above, the acrylate/methacrylate systems were investigated at $23{ }^{\circ} \mathrm{C}$ within this work. First, the terminal model and the penultimate unite model will be described, as these are the models most frequently used when describing copolymer kinetics. Then, these models will be applied to describe and fit experimental results. 


\subsection{Propagation in Copolymerization}

\subsubsection{Kinetic MOdels FOR Free-RAdical Copolymerization}

Many attempts have been made to describe copolymerization. Copolymerization models are used to predict overall propagation rate coefficients of copolymerizations, the composition and sequence distribution of the resulting copolymer, as a function of the feed ratio of the comonomers and a small set of characteristic constants. A short survey on the kinetic schemes and equations involved will be presented, starting with the simple approach of the terminal model.

\subsubsection{TERMINAL MODEL}

The basic kinetic model describing propagation in copolymerization was developed in the early 1940s $[9,10]$. It has been used frequently since then. The terminal model considers only the influence of the last (terminal) unit in a macroradical on the propagation rate and copolymer composition, it is assumed that side reactions are not significant. As a result, there are only four types of propagation reactions in free-radical copolymerization of any two given monomers:

$$
\mathrm{R}-\mathrm{M}_{\mathrm{i}} \cdot+\mathrm{M}_{\mathrm{j}} \stackrel{k_{\mathrm{pij}}}{\longrightarrow} \mathrm{R}-\mathrm{M}_{\mathrm{i}} \mathrm{M}_{\mathrm{j}} \cdot \quad \text { where } \mathrm{i}, \mathrm{j}=1 \text { or } 2
$$

The following monomer reactivity ratios ( $r$ values) can be defined:

$$
r_{\mathrm{i}}=\frac{k_{\mathrm{pii}}}{k_{\mathrm{pij}}} \quad \text { where } \mathrm{i}, \mathrm{j}=1 \text { or } 2 \text { and } \mathrm{i} \neq \mathrm{j}
$$

These parameters are used to calculate the copolymer composition in a system with two monomers at infinitely low conversions. Eq. 5.1 has been termed "Lewis-Mayo equation".

$$
\frac{F_{1}}{F_{2}}=\frac{\mathrm{d} c_{\mathrm{M}_{1}}}{\mathrm{~d} c_{\mathrm{M}_{2}}}=\frac{f_{1} \cdot\left(r_{1} \cdot f_{1}+f_{2}\right)}{f_{2} \cdot\left(r_{2} \cdot f_{2}+f_{1}\right)} \quad \text { or } \quad F_{1}=\frac{r_{1} \cdot f_{1}^{2}+f_{1} \cdot f_{2}}{r_{1} \cdot f_{1}^{2}+2 \cdot f_{1} \cdot f_{2}+r_{2} \cdot f_{2}^{2}}
$$

$f_{\mathrm{i}}$ : $\quad$ mole fraction of component $\mathrm{i}$ in the monomer mixture

$F_{\mathrm{i}}$ : mole fraction of component $\mathrm{i}$ in the copolymer

For a two-monomer system, an expression for the overall copolymer propagation rate coefficient has been derived: 


$$
k_{\mathrm{p}, \mathrm{copo}}=\frac{r_{1} \cdot f_{1}^{2}+r_{2} \cdot f_{2}^{2}+2 \cdot f_{1} \cdot f_{2}}{\left(r_{1} \cdot f_{1} / k_{\mathrm{p} 11}\right)+\left(r_{2} \cdot f_{2} / k_{\mathrm{p} 22}\right)}
$$

Where $k_{\mathrm{p} 11}$ and $k_{\mathrm{p} 22}$ denote the homopropagation rate coefficients.

With the advances of experimental techniques to measure the average or combined propagating rate coefficient $k_{\mathrm{p}, \text { copo }}$ as a function of the monomer feed composition, such as PLP-SEC, deviations from the terminal model were found. It become clear that in most cases the terminal model is not capable of describing both the copolymer composition and the propagation kinetics with one set of reactivity ratios. Although it has been shown by Maxwell et al. [11] that only one extra parameter is needed for an adequate fit of both sets, the penultimate unite model with two additional parameters has been chosen to represent copolymerization rate coefficients, $k_{\mathrm{p}, \text { copo }}$.

\subsubsection{EXPLICIT AND IMPLICIT PENULTIMATE UNIT MODELS}

The most widely used model is the so called "penultimate unit effect model" (PUE). The penultimate model assumes that the radical reactivity is affected by the preceding unit on the chain, that is, the radical $R_{i j}$ is distinguished from the radical $R_{j j}$ and both differ in their reactivity towards the monomer. As in the terminal model, side reactions (such as chain transfer to monomer or chain transfer to polymer) are considered insignificant. In the penultimate unite model kinetic scheme becomes more complex:

$$
\mathrm{R}-\mathrm{M}_{\mathrm{i}} \mathrm{M}_{\mathrm{j}} \cdot+\mathrm{M}_{\mathrm{k}} \stackrel{k_{\mathrm{pijk}}}{\longrightarrow} \mathrm{R}-\mathrm{M}_{\mathrm{i}} \mathrm{M}_{\mathrm{j}} \mathrm{M}_{\mathrm{k}} \cdot \quad \text { where } \mathrm{i}, \mathrm{j}, \mathrm{k}=1 \text { or } 2
$$

For a two-monomer system, there are eight propagation reactions, with the rate coefficient $k_{\mathrm{pijk}}$ representing the reaction of radical $\mathrm{R}_{\mathrm{ij}}$ with a monomer $\mathrm{M}_{\mathrm{k}}$. The reactivity ratios are defined as follows:

$$
r_{\mathrm{ii}}=\frac{k_{\mathrm{piii}}}{k_{\mathrm{piij}}} ; \quad r_{\mathrm{ij}}=\frac{k_{\mathrm{pijj}}}{k_{\mathrm{piji}}} ; \quad s_{\mathrm{i}}=\frac{k_{\mathrm{pjii}}}{k_{\mathrm{piii}}}
$$

where $i, j=1$ or 2 and $i \neq j$

The radical reactivity ratios, $s_{\mathrm{i}}$, represent the effect of the penultimate unit on the addition rate of a monomer $\mathrm{M}_{\mathrm{i}}$ to a radical $\mathrm{R}_{\mathrm{ii}}$ or $\mathrm{R}_{\mathrm{ji}}$. The relation between the penultimate and terminal reactivity ratios is given by Eqs. 5.3 and 5.4 [12]:

$$
\overline{r_{\mathrm{i}}}=r_{\mathrm{ji}} \cdot \frac{r_{\mathrm{ii}} \cdot f_{\mathrm{i}}+f_{\mathrm{j}}}{r_{\mathrm{ji}} \cdot f_{\mathrm{i}}+f_{\mathrm{j}}}
$$




$$
\overline{k_{\text {pii }}}=k_{\text {piii }} \cdot \frac{r_{\text {ii }} \cdot f_{\mathrm{i}}+f_{\mathrm{j}}}{r_{\mathrm{ii}} \cdot f_{\mathrm{i}}+f_{\mathrm{j}} / s_{\mathrm{i}}}
$$

where $k_{\text {piii }}$ denotes the respective homopropagation rate coefficient.

The corresponding expressions for copolymer composition and overall propagation rate coefficients can be deduced by substituting Eq. 5.3 and Eq. 5.4 into Eq. 5.1 (by identifying $r_{\mathrm{i}}$ with $\overline{r_{\mathrm{i}}}$ ) and 5.2 (by identifying $k_{\mathrm{pii}}$ with $\overline{k_{\mathrm{pii}}}$ ), respectively. A few systems (such as styrene/acrylonitrile and p-chlorostyrene/methyl acrylate) have been found to this date $[13,14]$, where the copolymer composition and sequence distribution (triad sequences) cannot be adequately described by the terminal model. Fukuda and co-workers coined the phrase "explicit penultimate unit effect" (EPUE) to describe these systems, which require the full set of eight propagation rate coefficients to describe the copolymer composition and rate. In most cases $[12,15,16]$, however, the copolymer composition is well described by the terminal model, but not the overall propagation rate coefficient. In this case, two simplifications can be made:

$$
r_{\mathrm{ii}}=r_{\mathrm{ji}}=\bar{r}_{\mathrm{i}} \quad \text { and } \quad r_{\mathrm{jj}}=r_{\mathrm{ij}}=\bar{r}_{\mathrm{j}}
$$

These systems are said to display an "implicit penultimate unit effect" (IPUE) [17]. For all these systems, measured copolymerization rate coefficients, $k_{\mathrm{p}, \text { copo }}$, are lower than predicted by the terminal model. Based upon stabilization energy arguments, Fukuda et al. [18] suggested that

$$
\overline{r_{\mathrm{i}}} \cdot \overline{r_{\mathrm{j}}}=s_{\mathrm{i}} \cdot s_{\mathrm{j}}
$$

Since it is often difficult to obtain accurate estimates of both $s$ values from experimental data, Fukuda and co-workers [18] also suggested the simplification $s_{1}=s_{2}$. This immediately leads to:

$$
S_{\mathrm{i}}=S_{\mathrm{j}}=\left(\overline{r_{\mathrm{i}}} \cdot \overline{r_{\mathrm{j}}}\right)^{0.5}
$$

It has been demonstrated that these simplifications do a reasonable job in describing the experimental data in some cases [19].

The penultimate unit model, which traces back to Merz et al. [20], has received considerable attention over the past decade, which is primarily due to the convincing copolymerization experiments of Fukuda and co-workers [21]. The particularly attractive feature of the penultimate unit model is that previously determined monomer reactivity ratios from the terminal model fittings to composition data (see below) may be further utilized. It is for this 
reason that other models (which certainly have their place in copolymerization kinetics) are not considered in bulk free-radical copolymerizations. Thus, the present work will focus on the interpretation of experimental PLP-SEC data by the implicit penultimate unit effect model.

A growing body of evidence shows that in practice it is very difficult, if not impossible, to accurately determine all four reactivity ratios of the IPUE model just from composition and rate data $[22,23,24]$. This task is even more difficult for systems displaying an EPUE. In this work it will be demonstrated that for each individual copolymerizing system a careful data analysis is needed to derive definite statements about reactivity ratio data. The overall strategy is straightforward:

1. The system under investigation is analyzed with respect to copolymer composition by ${ }^{1} \mathrm{H}-\mathrm{NMR}$ spectroscopy and the overall propagation rate coefficient is determined by PLP-SEC experiments at different initial monomer feed ratios. In case that no literature data on the Mark-Houwink parameters, $a$ and $K$, are available, these quantities, which are required for SEC analysis, have also been determined at different copolymer compositions, too.

2. The two experimental data sets, copolymer composition and overall propagation rate coefficient, are fitted simultaneously to derive the variables $r_{\mathrm{ij}}, s_{\mathrm{j}}$. The following error function is usually used:

$$
\text { error }=\sum \sqrt{\left(1-\frac{d a t a^{\text {measured }}}{d a t a^{\text {mod el }}}\right)^{2}}
$$

3. The least squares fitting procedure according to Levenberg-Marquardt [25,26] is started and the program provides a set of (optimum) parameters. However, these parameters need not constitute the only solution. It turns out, that the $r$ values are usually close or identical to those determined by the Lewis-Mayo procedure [27] and independent of the starting values selected for the data fitting procedure. The $s$ values are not as easily determined.

For a description of copolymerization behavior, many studies have been devoted to the determination of accurate and precise reactivity ratios. The usual way to obtain the values of the parameters in copolymerization is to measure copolymer composition via ${ }^{1} \mathrm{H}-\mathrm{NMR}$ with subsequent fitting of the experimental obtained composition data to the terminal composition equation (Eq. 5.1). Once these data are obtained, they are sufficient to distinguish between a number of models described above. 
In the course of this work, the simultaneous fitting procedure outlined above is used for composition and rate data analysis. The results are presented for each individual copolymerization system.

\subsubsection{DETERMINATION OF COPOLYMER COMPOSITION BY ${ }^{1}$ H-NMR SPECTROSCOPY}

This section deals with the determination and interpretation of (terminal model) reactivity ratios, $r$. Copolymer composition was determined for different acrylate/methacrylate copolymer systems. Literature data is available only for a part of these systems. The results of these investigations are summarized in Tab. 5.1.

\begin{tabular}{cccccc}
\hline System & $\Theta /{ }^{\circ} \mathrm{C}$ & $p /$ bar & $r_{\mathrm{ac}}$ & $r_{\mathrm{mac}}$ & Source \\
\hline MA/MMA & 50 & 1 & 0.42 & 2.36 & {$[28]$} \\
MA/MMA & 23 & 1000 & 0.20 & 3.03 & this work \\
\hline MA/DMA & 40 & 1000 & 0.25 & 2.66 & this work \\
MA/DMA & 40 & 1000 & 0.21 & 2.37 & {$[29]$} \\
\hline DA/MMA & 23 & 1000 & 0.29 & 2.42 & this work \\
DA/MMA & 40 & 1000 & 0.43 & 3.26 & {$[29]$} \\
\hline BA/MMA & 50 & 1 & 0.41 & 2.24 & {$[30]$} \\
DA/DMA & 40 & 1000 & 0.38 & 2.21 & this work \\
\hline
\end{tabular}

Tab. 5.1 Terminal model reactivity ratios for different copolymerization systems and experimental conditions of copolymer synthesis

The evaluation of ${ }^{1} \mathrm{H}-\mathrm{NMR}$ spectra will be demonstrated taking the copolymerization of DA and MMA as an example. Fig. 5.1 shows a typical ${ }^{1}$ H-NMR spectrum of a DA/MMA copolymer ( 8 mol \% MMA in the copolymer). The numbers assigned to each peak group in Fig. 5.1 refer to the proton positions indicated in the structural formula [31]. 


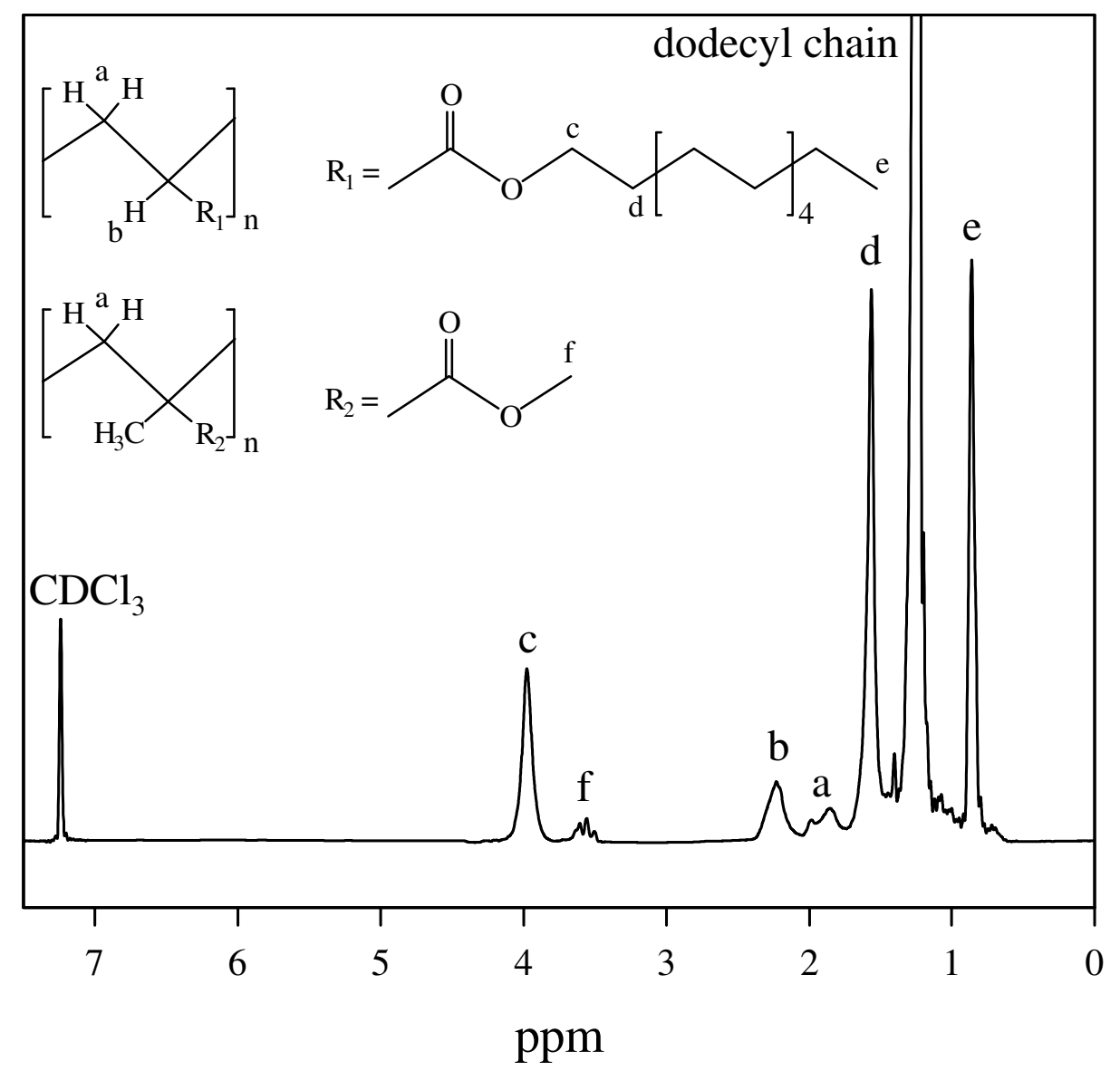

Fig. 5.1 ${ }^{1}$ H-NMR spectrum of a DA/MMA copolymer synthesized at $23{ }^{\circ} \mathrm{C}$ and 1000 bar. The individual peak assignments are explained in the text.

For the determination of the copolymer composition, peaks $\mathrm{c}$ and $\mathrm{f}$ are considered. They correspond to the methylene protons next to the oxygen in the ester chain of dodecyl acrylate (peak c) and the methyl ester group in methyl methacrylate (peak f). Peaks a and d are associated with protons at the backbone of the polymer chain for both monomers and the methylene group in $\beta$-position to the oxygen in the ester chain of dodecyl acrylate, respectively. Peak e corresponds to the terminal methyl group in the dodecyl ester rest, peak b corresponds to a proton at the backbone dodecyl acrylate unit of the polymer. Peak $\mathrm{f}$ is integrated and the resulting value is, compared to the value of the integral of peak c (which allows to deduce the equivalent of one proton) as an indirect measure of the mole fraction of MMA in the copolymer as given by Eq. 5.7:

$$
F_{\mathrm{MMA}}=\frac{\frac{\operatorname{Int}[\mathrm{f}]}{3}}{\frac{\operatorname{Int}[\mathrm{f}]}{3}+\frac{\operatorname{Int}[\mathrm{c}]}{2}}
$$


which can be rearranged to Eq. 5.8:

$$
F_{\mathrm{MMA}}=\frac{2 \operatorname{Int}[\mathrm{f}]}{2 \operatorname{Int}[\mathrm{f}]+3 \operatorname{Int}[\mathrm{c}]}
$$

The results from the ${ }^{1} \mathrm{H}-\mathrm{NMR}$ experiments (Tab. 5.2) are shown in Fig. 5.2. Plotted is the mole fraction of MMA in the copolymer vs. the mole fraction of MMA in the comonomer feed.

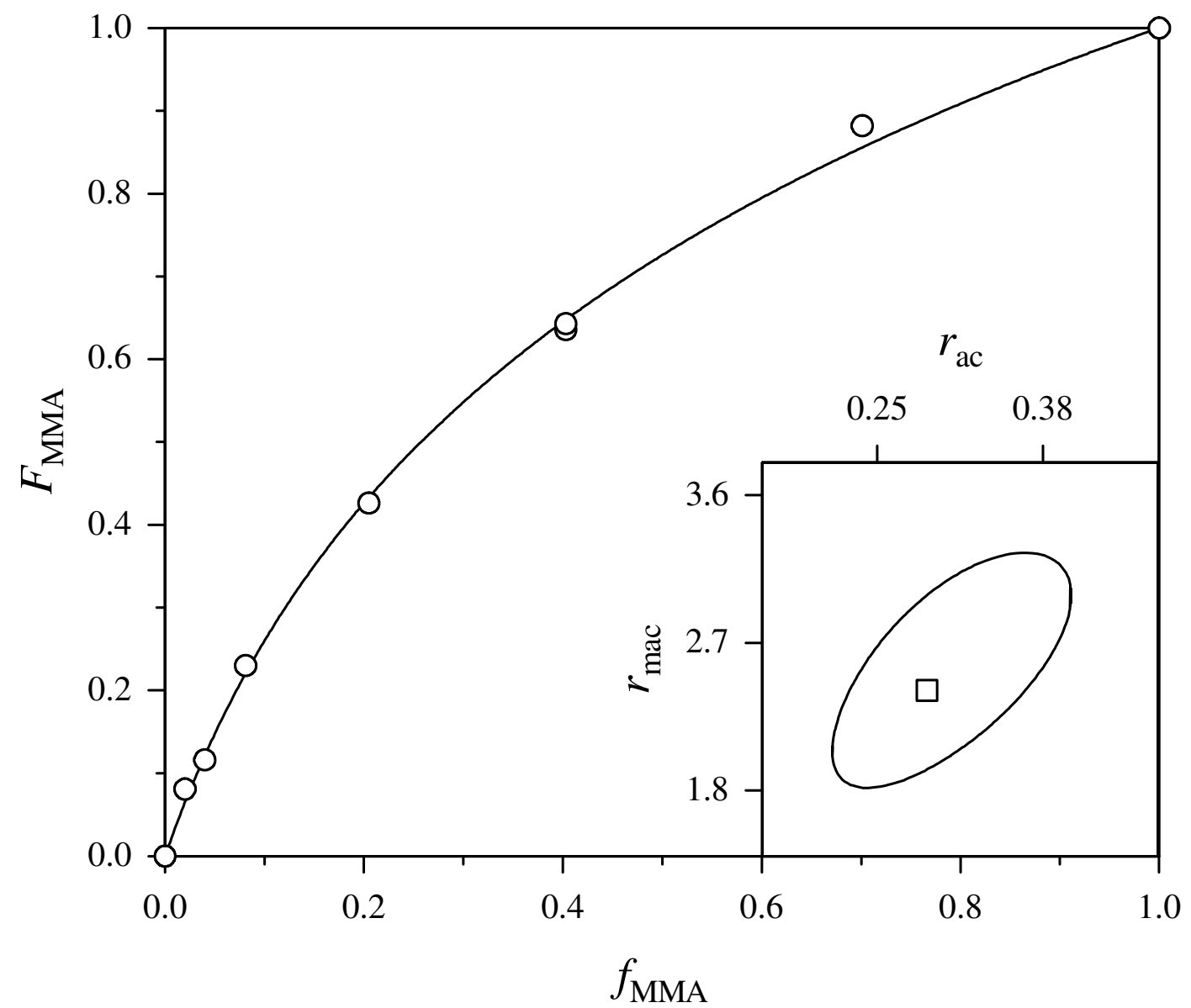

Fig. 5.2 Lewis-Mayo plot for the DA/MMA copolymerization at $23{ }^{\circ} \mathrm{C}$ and 1000 bar; $95 \%$ joint confidence interval for the reactivity ratios ( $r$ values) is given.

It is clearly seen that the MMA content of the polymer material always exceeds the MMA content of the associated monomer mixture (at differential conversion). This phenomenon may be explained in terms of radical stabilization: Propagating chains that carry a MMA unit in the terminal position are more stable than chains that carry a DA unit in the terminal position. The $\alpha$-methyl group in methacrylate stabilizes the radical by increasing the electron density. In many cases, the more stable product is also the one that is formed faster [32]. A propagating radical, having either DA or MMA as the terminal unit, has the tendency to react 
faster with a MMA monomer than with a DA monomer, due to the fact that the reaction path to the more stable product should also be the kinetically favored path. In other words, the activation energy for the reaction of the same propagating chain with a MMA monomer is always lower than the activation energy of the corresponding reaction with a DA monomer. This explanation [33] may hold true for all acrylate/methacrylate copolymerizations listed in Tab. 5.1.

Fig. 5.3 plots the entire set of copolymerization data from Tab. 5.2 and [29] in a single $F_{\text {mac }}$ vs. $f_{\text {mac }}$ diagram.

\begin{tabular}{|c|c|c|c|}
\hline \multicolumn{2}{|c|}{ MA/MMA } & \multicolumn{2}{|c|}{ DA/MMA } \\
\hline$f_{\mathrm{mac}}$ & $F_{\text {mac }}$ & $f_{\text {mac }}$ & $F_{\text {mac }}$ \\
\hline 0.025 & 0.123 & 0.020 & 0.081 \\
\hline 0.081 & 0.268 & 0.040 & 0.116 \\
\hline 0.205 & 0.493 & 0.081 & 0.230 \\
\hline 0.404 & 0.760 & 0.205 & 0.426 \\
\hline \multirow[t]{3}{*}{0.701} & 0.859 & 0.403 & 0.636 \\
\hline & & 0.403 & 0.643 \\
\hline & & 0.701 & 0.882 \\
\hline
\end{tabular}

Tab. 5.2 Copolymer methacrylate contents determined by ${ }^{1} \mathrm{H}-\mathrm{NMR}, F_{\text {mac }}$, associated monomer feed composition, $f_{\text {mac }}$, for copolymerizations of DA/MMA and MA/MMA obtained at $23{ }^{\circ} \mathrm{C}$ and 1000 bar.

The composition data of these acrylate/methacrylate copolymerizations are reasonably well fitted by one curve. The particular type of acrylate and methacrylate monomer appears to have no significant influence on the $F_{\mathrm{mac}}-f_{\mathrm{mac}}$ correlation. The difference in copolymerization temperature also does not seem to influence the $F_{\text {mac }}-f_{\text {mac }}$ correlation. The full line in Fig. 5.3 represents the fit of the entire data set to the Lewis-Mayo copolymerization equation (Eq. 5.1). The methacrylate and acrylate reactivity ratios referring to the fitted line in Fig. 5.3 are $r_{\mathrm{mac}}=2.55$ and $r_{\mathrm{ac}}=0.29$. 


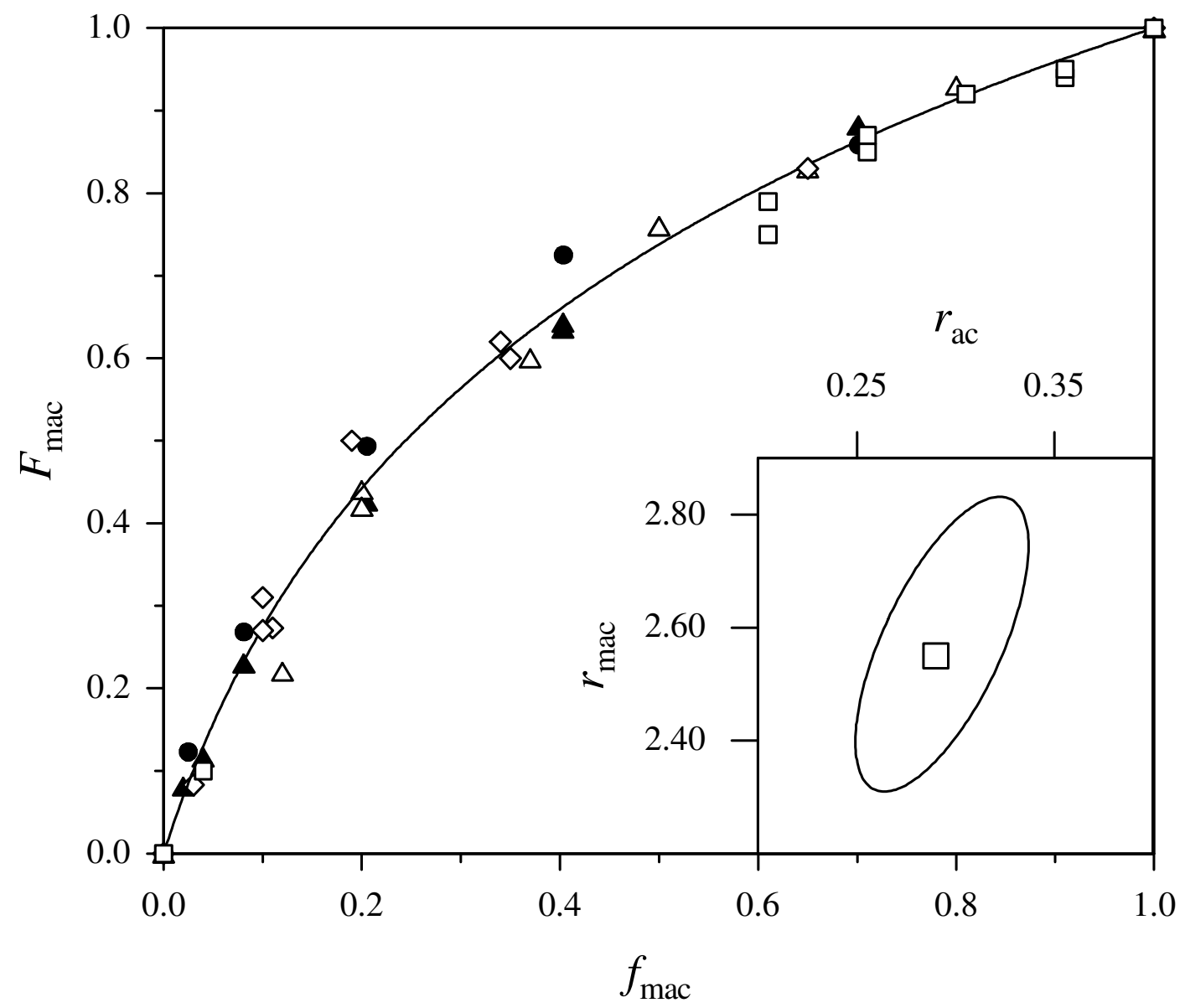

Fig. 5.3 Lewis-Mayo plot for all copolymerization systems under investigation: DA/DMA $\left(40{ }^{\circ} \mathrm{C}\right.$, squares), MA/DMA $\left(40{ }^{\circ} \mathrm{C}\right.$, diamonds), DA/MMA $\left(40{ }^{\circ} \mathrm{C}\right.$, triangles), DA/MMA $\left(23{ }^{\circ} \mathrm{C}\right.$, full triangles $)$, and MA/MMA $\left(23{ }^{\circ} \mathrm{C}\right.$, full circles); $95 \%$ joint confidence interval for the reactivity ratios ( $r$ values) is given. The full line represents the fit of the entire data set to the Lewis-Mayo copolymerization equation (Eq. 5.1). The composition data refers to 1000 bar.

\subsubsection{EXPerimental Results ANd Data Analysis by PUE Models}

Studies into acrylate/methacrylate systems are of particular interest as the homopropagation rate coefficients of the two families differ significantly, by about 1 to 2 orders of magnitude $[30,33,34]$. The propagation rate coefficients for these systems were determined by the PLPSEC technique, where pulsed laser induced polymerization is combined with chromatographic analysis of the polymeric product.

The present work focuses on the measurement of $k_{\mathrm{p}, \text { copo }}$ data for the systems MA/MMA, MA/DMA, DA/MMA, and DA/DMA obtained at 22 to $40{ }^{\circ} \mathrm{C}$ and 1000 bar. The systems 
were chosen such as to contain acrylates and methacrylates of both small (methyl) and long (dodecyl) ester size. The systems MA/DMA and DA/DMA at $40{ }^{\circ} \mathrm{C}$ and 1000 bar have partly been measured by Buback et al. [29].

\subsubsection{METHYL ACRYLATE / METHYL METHACRYLATE}

In this section results obtained for copolymerization of MA/MMA measured at $23{ }^{\circ} \mathrm{C}$ and 1000 bar are presented. No Mark-Houwink parameters, $a$ and $K$, were available for this system. They were determined at five copolymer compositions evenly distributed between the two homopolymers. From this data, intermediate $a$ and $K$ values were deduced. A careful data analysis is needed to decide on a method for this interpolation. The methods employed are described below and their impact on the resulting copolymerization propagation rate coefficients is discussed.

\begin{tabular}{cccc}
\hline$F_{\text {MMA }}$ & $a$ & $K / \mathrm{mL} \mathrm{g}^{-1}$ & Source \\
\hline 1.000 & 0.719 & 0.00944 & {$[30]$} \\
0.951 & 0.664 & 0.03120 & this work \\
0.820 & 0.676 & 0.02670 & this work \\
0.643 & 0.667 & 0.02880 & this work \\
0.397 & 0.683 & 0.02280 & this work \\
0.119 & 0.603 & 0.06450 & this work \\
0.000 & 0.696 & 0.01680 & {$[29]$} \\
\hline
\end{tabular}

Tab. 5.3 Mark-Houwink parameters $a$ and $K$ at $30{ }^{\circ} \mathrm{C}$ with THF as eluent for MA/MMA copolymers. Copolymer composition was determined by ${ }^{1} \mathrm{H}-\mathrm{NMR}$ spectroscopy. Parameters are valid for copolymers ranging from 10000 to $1000000 \mathrm{~g} \cdot \mathrm{mol}^{-1}$.

The calibration procedure via the Mark-Houwink coefficients (Eq. 3.17), that are determined in a separate set of experiments, will now be outlined. Mark-Houwink parameters for both homopolymers are available in the literature [29,30]. When preparing samples for the determination of Mark-Houwink parameters, great care has to be taken to produce broad MWDs. Furthermore, it must be guaranteed that the copolymers produced by the PLP procedure are in the same molecular weight range as the copolymer samples used for Mark- 
Houwink parameters determination. The parameters given in this work can be used at molecular weights ranging from 10000 to 1000 000. Tab. 5.3 gives the parameters $a$ and $K$ together with the respective copolymer composition of the system MA/MMA.

For the analysis of the data set given in Tab. 5.3 two methods are employed that allow to calculate Mark-Houwink coefficients at copolymer compositions different from those determined experimentally. The first approach is independent of the Mark-Houwink coefficients determined experimentally from copolymeric products: a simple linear interpolation between the homopolymer values was chosen to represent the intermediate values. This is indicated by the full lines in Figs. 5.4b and 5.4c. Propagation rate coefficients resulting from this treatment are represented by the open triangle symbols in Fig. 5.4a. Finally, the individual data points have been connected by a simple spline function and the Mark-Houwink parameters corresponding to intermediate compositions are calculated by linear interpolation between two measured data points (dotted lines). The resulting $k_{\mathrm{p}, \text { copo }}$ data set is represented by open diamonds.

The dependence of $a$ and $K$ on $F_{\text {MMA }}$ demonstrates the high degree of correlation between both quantities. Unfortunately, values of $a$ and $K$ measured by PSS and analyzed within this work are inconsistent with the values reported in the literature for homopolymers and therefore cannot be used for calculation of $k_{\mathrm{p}, \mathrm{copo}}$. For illustration, the intrinsic viscosity, $[\eta]$, recalculated from Eq. 3.16 as a function of $F_{\mathrm{MMA}}$ in Fig. 5.5 is given.

Values of $a$ and $K$ obtained from linear interpolation of the homopolymer numbers provide data which allow to make reasonable estimates of copolymer molecular weights. Within the present work for the system MA/MMA, the linear interpolation procedure between the homopolymer values is used to estimate copolymer Mark-Houwink constants. 


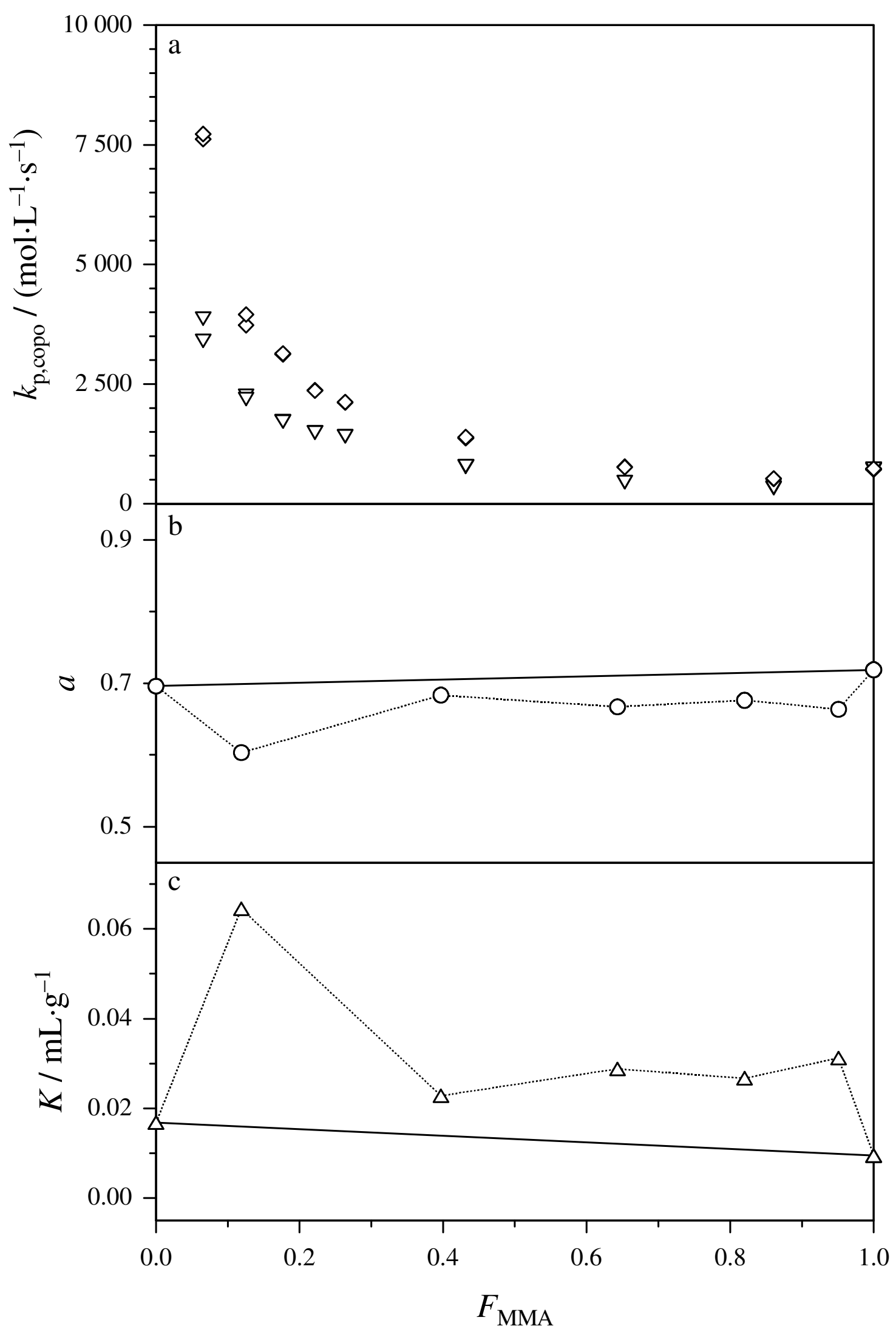

Fig. 5.4 Mark-Houwink data for the MA/MMA system (for additional information see next page) 
Additional information to Fig. 5.4:

5.4a Propagation rate coefficients, $k_{\mathrm{p}, \mathrm{copo}}$, resulting from different methods of fitting the Mark-Houwink coefficients $a$ and $K$. Linear spline interpolation (open diamonds); linear interpolation between homopolymer values (open triangles)

5.4b Mark-Houwink coefficient $a$ vs. copolymer composition, $F_{\mathrm{MMA}}$, fitted by different methods. Linear spline interpolation (dotted line); linear interpolation between homopolymer values (full line)

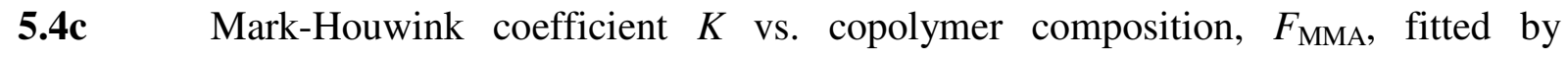
different methods. Linear spline interpolation (dotted line); linear interpolation between homopolymer values (full line)

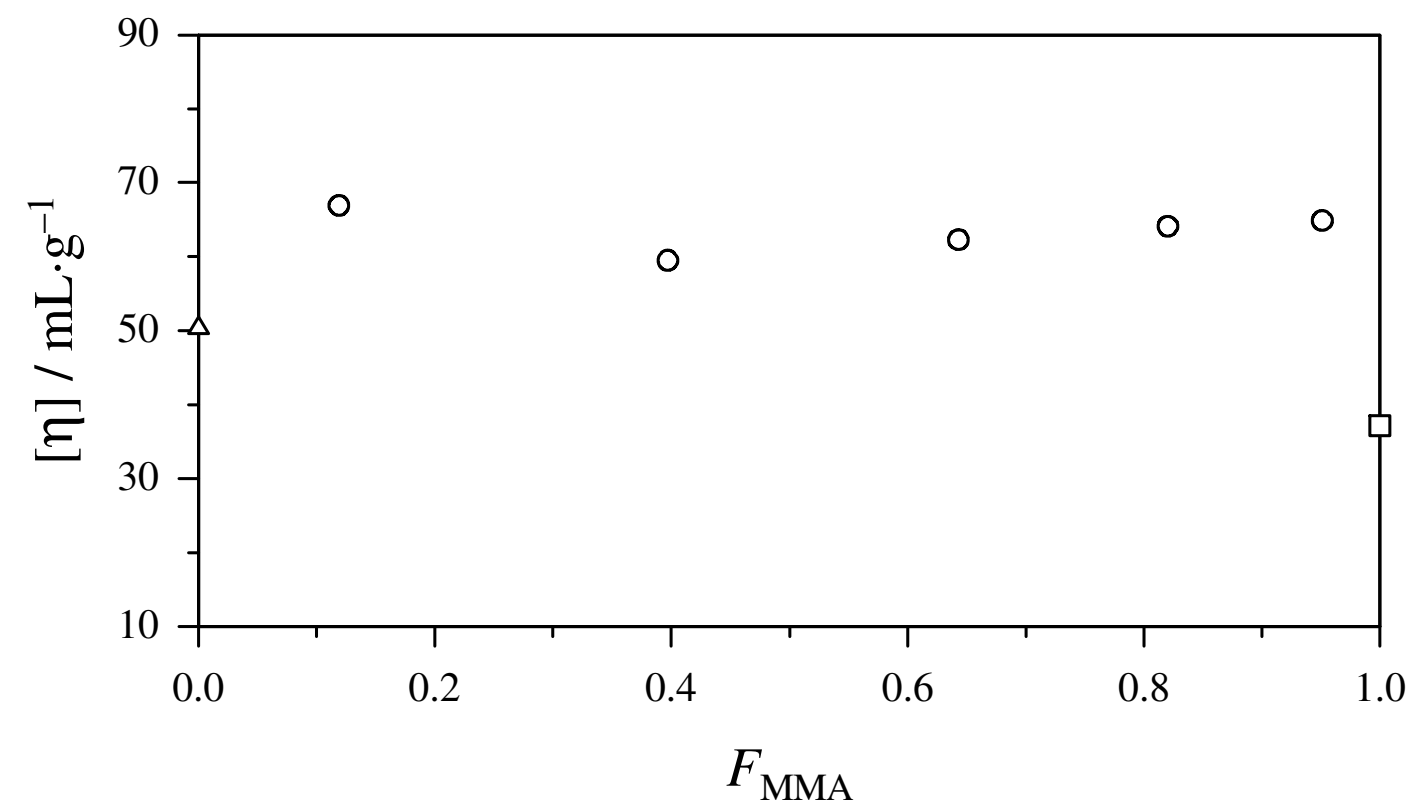

Fig. 5.5 Intrinsic viscosity, $[\eta]$, recalculated with Mark-Houwink parameters for MA ([29], triangle), MMA ([30], square) and several copolymers ( $a$ and $K$ obtained by PSS, circles) via Eq. $3.16, M=10^{5} \mathrm{~g} \cdot \mathrm{mol}^{-1}$.

Fig. 5.6 shows an experimental molecular weight distribution at an initial monomer feed of $70.0 \mathrm{~mol} \% \mathrm{MMA}$ generated at $23^{\circ} \mathrm{C}$ and 1000 bar. The data set is already recalibrated by the Mark-Houwink coefficients. Four points of inflection on the MWD can be clearly identified as maxima in the first derivative curve (dashed line), indicating that the formation of the molecular weight distribution was controlled by laser pulsing. The laser frequency was $70 \mathrm{~Hz}$ in this particular case. Particular attention was paid to the region with high amount of MA $\left(f_{\mathrm{MA}}>0.9\right)$, because the largest change in the overall propagation rate coefficient is to be expected here, as will be detailed below. 


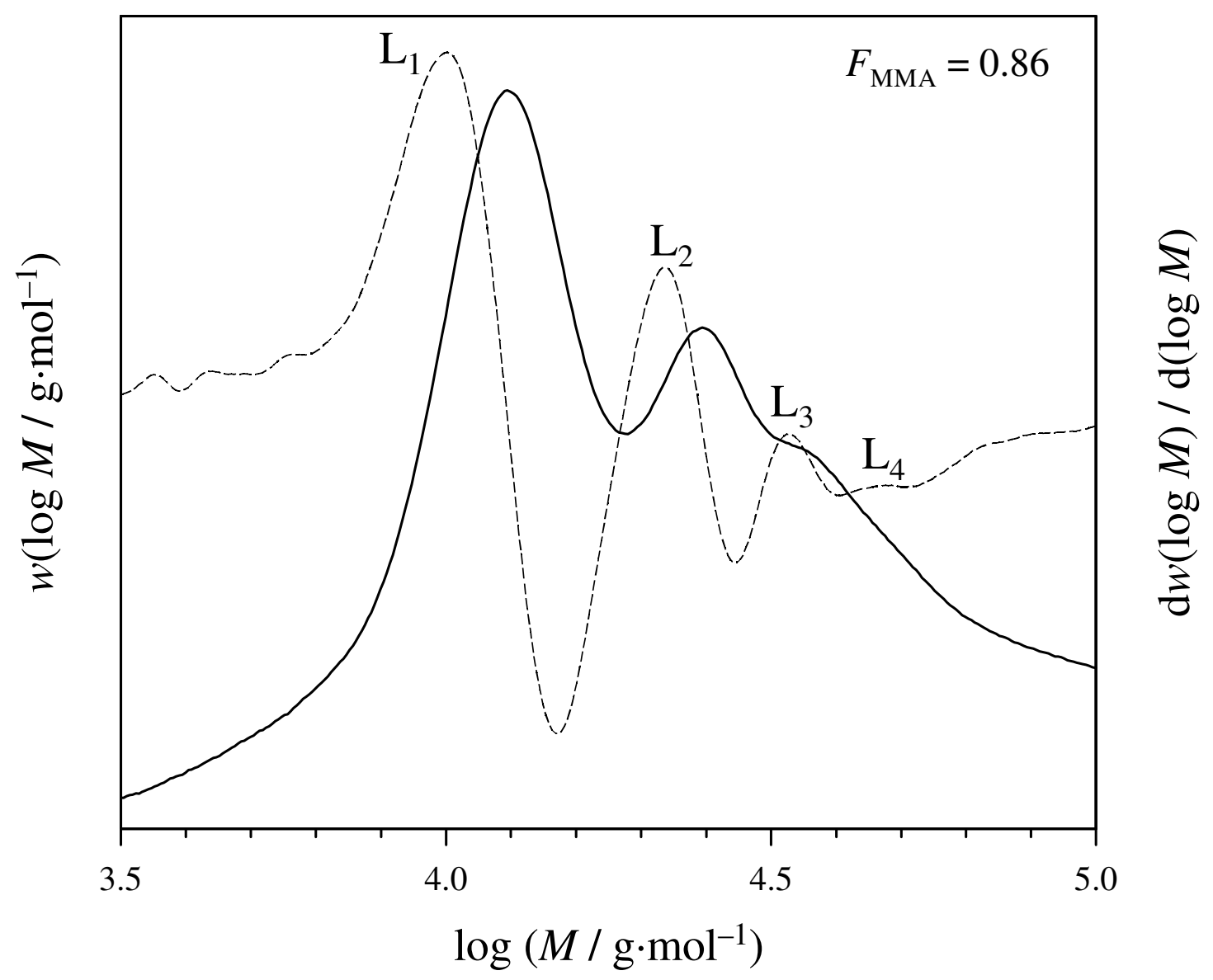

Fig. 5.6 Experimental molecular weight distribution (full line) of a MA/MMA copolymer produced at $23{ }^{\circ} \mathrm{C}$ and 1000 bar using a pulse repetition rate of $70 \mathrm{~Hz}$. The first derivative of the MWD is also given (dashed line). Clearly seen are the higher order inflection points $\left(\mathrm{L}_{2}\right.$ to $\left.\mathrm{L}_{4}\right)$, which serve as a consistency check of the PLPSEC experiment.

The parameters of the PLP-SEC experiments and the resulting $k_{\mathrm{p}}$ values for the entire data set (together with the pulse frequency and the inflection points used for $k_{\mathrm{p}, \text { copo }}$ determination) are given in Tab. 5.4.

Only those molecular weight distributions were considered in the kinetic analysis, for which the first derivative clearly meets the consistency criteria $\left(\mathrm{L}_{2}=2 \cdot \mathrm{L}_{1}\right)$. 


\begin{tabular}{ccccc}
\hline$f^{0}{ }_{\mathrm{MMA}}$ & $v / \mathrm{Hz}$ & $M_{\mathrm{L} 1} / \mathrm{g} \mathrm{mol}^{-1}$ & $M_{\mathrm{L} 2} / \mathrm{g} \mathrm{mol}^{-1}$ & $k_{\mathrm{p}} / \mathrm{L} \mathrm{mol}^{-1} \mathrm{~s}^{-1}$ \\
\hline 1.00 & 40 & 16853 & 32228 & 715 \\
1.00 & 40 & 17225 & 33007 & 731 \\
0.70 & 70 & 9663 & 21071 & 717 \\
0.70 & 70 & 9795 & 21522 & 707 \\
0.40 & 70 & 14091 & 29463 & 736 \\
0.40 & 70 & 14294 & 29720 & 747 \\
0.20 & 100 & 17056 & 35691 & 840 \\
0.20 & 100 & 17246 & 36180 & 811 \\
0.10 & 100 & 29617 & 68837 & 1408 \\
0.10 & 100 & 29725 & 68721 & 1408 \\
0.08 & 100 & 33113 & 70738 & 1488 \\
0.08 & 100 & 33267 & 71120 & 1495 \\
0.06 & 100 & 38976 & 81452 & 1718 \\
0.06 & 100 & 38789 & 82156 & 1726 \\
0.04 & 100 & 49520 & 104754 & 2185 \\
0.04 & 100 & 52323 & 107649 & 2257 \\
0.02 & 100 & 83360 & 194984 & 3868 \\
0.02 & 100 & 82238 & 163678 & 3403 \\
0.00 & 100 & 181853 & 338057 & 17476 \\
0.00 & 100 & 194258 & 340463 & 18683 \\
\hline
\end{tabular}

Tab. 5.4 PLP-SEC results for the copolymerization of methyl acrylate and methyl methacrylate (MA/MMA) at $23{ }^{\circ} \mathrm{C}$ and 1000 bar.

Fig. 5.7 shows a plot of the $k_{\mathrm{p}, \text { copo }}$ data vs. the initial MMA mole fraction of the comonomer mixture. In MA/MMA copolymerization, $k_{\text {p,copo }}$ is almost constant at methacrylate concentrations above $f_{\text {mac }}=0.2$ and steeply increases at methacrylate mole fractions below $f_{\mathrm{mac}}=0.1$. The rapidly increasing $k_{\mathrm{p}, \mathrm{copo}}$ value in this reaction makes it extremely difficult (as has also been shown by [33]) to conduct successful PLP-SEC experiments at these conditions. The MA/MMA system has already been studied by $[28,35]$ at $50{ }^{\circ} \mathrm{C}$ and ambient pressure. The resulting $k_{\mathrm{p} \text {,copo }}$ data were analyzed with respect to the terminal and implicit penultimate unit effect model. The authors show that the free-radical copolymerization of MA/MMA at $50{ }^{\circ} \mathrm{C}$ can be well described by the IPUM. The terminal model failed to predict the kinetic behavior of this system.

The $k_{\mathrm{p} \text {,сopo }}$ values in this work are fitted by two procedures: (a) via the terminal model expression (Eq. 5.2) and (b) via the IPUE effect model by simultaneously fitting the $k_{\mathrm{p} \text {,copo }}$ and composition data. A data analysis by the IPUE effect model was carried out by the procedure outlined in section 5.1.1. 
The resulting data set was analyzed by the terminal model first. Although the terminal model failed in description the copolymerization of MA/MMA at $50{ }^{\circ} \mathrm{C}$ and ambient pressure [28], it describes the composition and propagation rate of this system at $23{ }^{\circ} \mathrm{C}$ and 1000 bar with almost the same reactivity ratios, as can be seen in Fig. 5.7.

It is thus to be expected that the IPUE fitting describes the system under investigation very well with $r_{\mathrm{mac}}$ and $r_{\mathrm{ac}}$ being close to the experimental values, and $s_{\mathrm{mac}}$ and $s_{\mathrm{ac}}$ being close to unity. The $r$ and $s$ values thus obtained are summarized in Tab. 5.11.

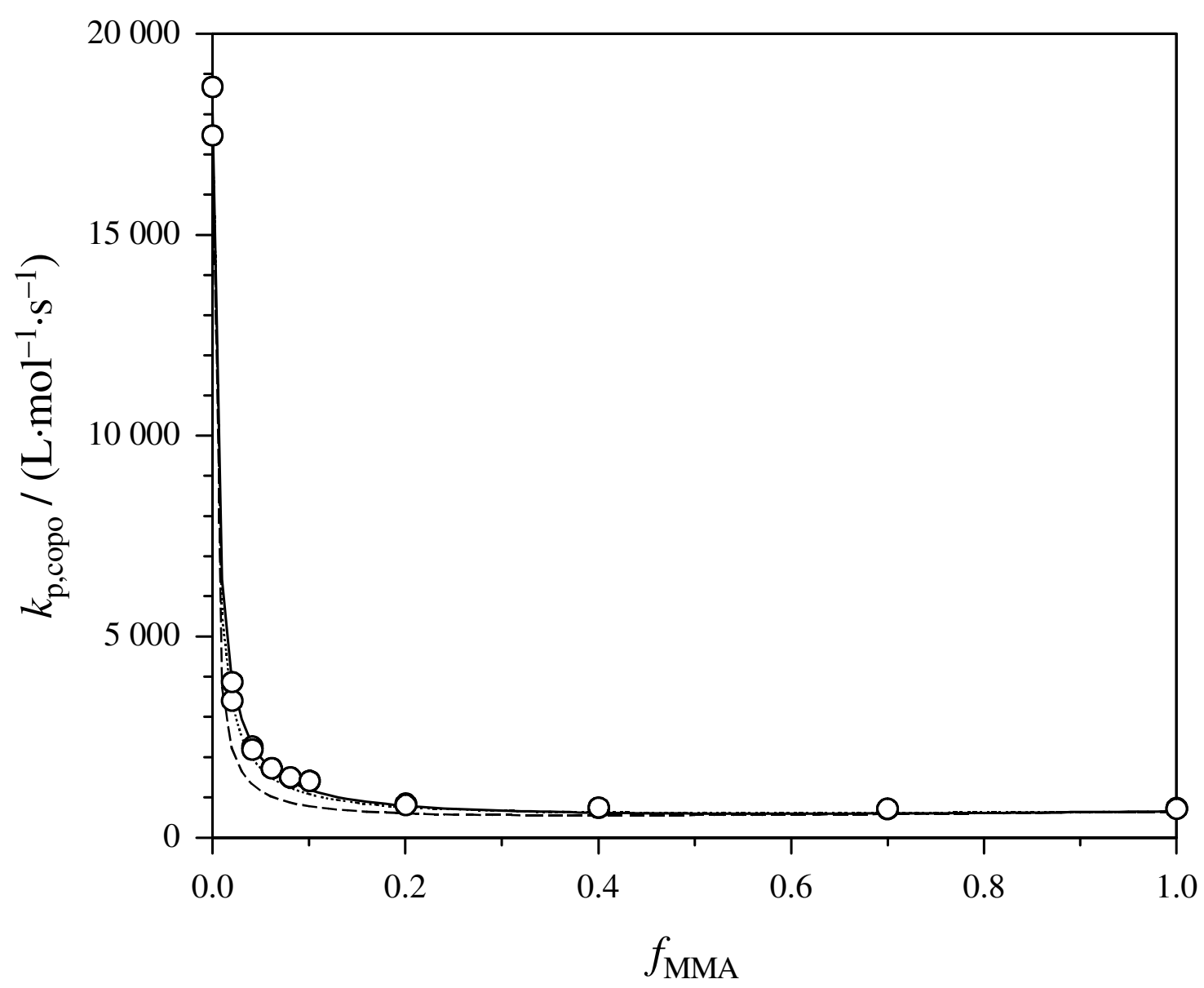

Fig. 5.7 Copolymerization propagation rate coefficients determined by PLP-SEC for the MA/MMA system as a function of the mole fraction of MMA in the monomer mixture $\left(f_{\mathrm{MMA}}\right)$ at $23{ }^{\circ} \mathrm{C}$ and 1000 bar. The full line corresponds to a fit according to the IPUE model as described in the text, the dotted line is a terminal model representation with the reactivity ratio data $r_{\mathrm{mac}}=2.55$ and $r_{\mathrm{ac}}=0.29$ taken from the Lewis-Mayo fit to the overall composition data (Fig. 5.3), the dashed line corresponds the terminal model fit to the composition data with reactivity ratios $r_{\mathrm{mac}}=3.03$ and $r_{\mathrm{ac}}=0.20$ (Tab. 5.1). 
It is rather typical for an acrylate/methacrylate copolymerization that the overall propagation rate coefficient is close to the methacrylate value over a wide range of initial monomer feed ratios. This observation has also been made for other systems, such as DA/DMA, DA/MMA and MA/DMA, as will be detailed below.

\subsubsection{DODECYL ACRYLATE / DODECYL METHACRYLATE}

For the copolymerization system DA/DMA at $23{ }^{\circ} \mathrm{C}$ a PLP-SEC study, similar to the one for the MA/MMA system, was performed to deduce $k_{\mathrm{p}, \text { copo }}$.

For this system, the Mark-Houwink parameters have already been reported [29].

As compared to the MA/MMA system, rather high pulse repetition rates $(100 \mathrm{~Hz})$ have to be used to generate a PLP-structure in the molecular weight distribution. The proper selection of the pulse laser rate plays an important role. The underlying arguments will not be presented here. An excellent survey on this issue has been given by Schweer [36] and Beuermann et al. [37]. In order to obtain well pronounced MWDs at very high initial mole fractions of DA in the monomer mixture (that is at $f_{\mathrm{DA}}>0.9$ ), the initiator DMPA was used at initial concentrations close to $2.5 \cdot 10^{-3} \mathrm{~mol} \cdot \mathrm{L}^{-1}$. This concentration is two times below the one, which is normally used in PLP-SEC experiments. Nicely structured molecular weight distributions are obtained in all cases. Fig. 5.7 shows the first derivatives of the MWD produced at $23{ }^{\circ} \mathrm{C}$ and 1000 bar at $100 \mathrm{~Hz}$.

Additionally, six values of $k_{\mathrm{p} \text {,copo }}$ at $f_{\mathrm{DA}}>0.9$ were added to the existed data for DA/DMA copolymerization at $40{ }^{\circ} \mathrm{C}$ and 1000 bar [29]. Fig. 5.9 presents the MWD and the associated first derivative curve of a DA/DMA copolymer $\left(f_{\mathrm{DMA}}=0.06\right)$ obtained at $40{ }^{\circ} \mathrm{C}$ and $1000 \mathrm{bar}$ by pulsed laser polymerization at a repetition rate of $100 \mathrm{~Hz}$. 


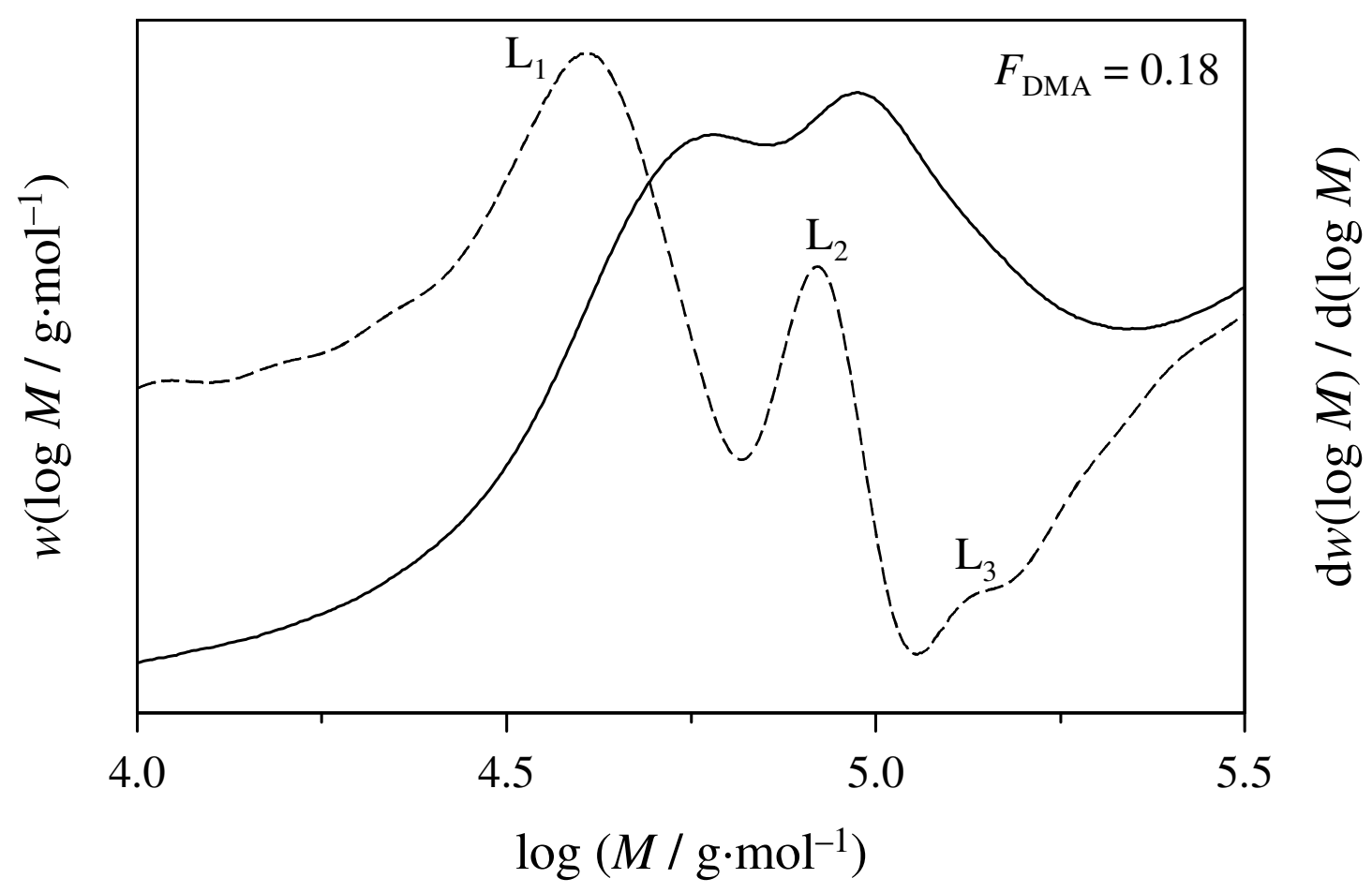

Fig. 5.8 Experimental MWD (full line) of a DA/DMA copolymer $\left(f_{\mathrm{DMA}}=0.06\right)$ produced at $23{ }^{\circ} \mathrm{C}$ and 1000 bar at $100 \mathrm{~Hz}$. A dashed line gives the associated first derivative curve.

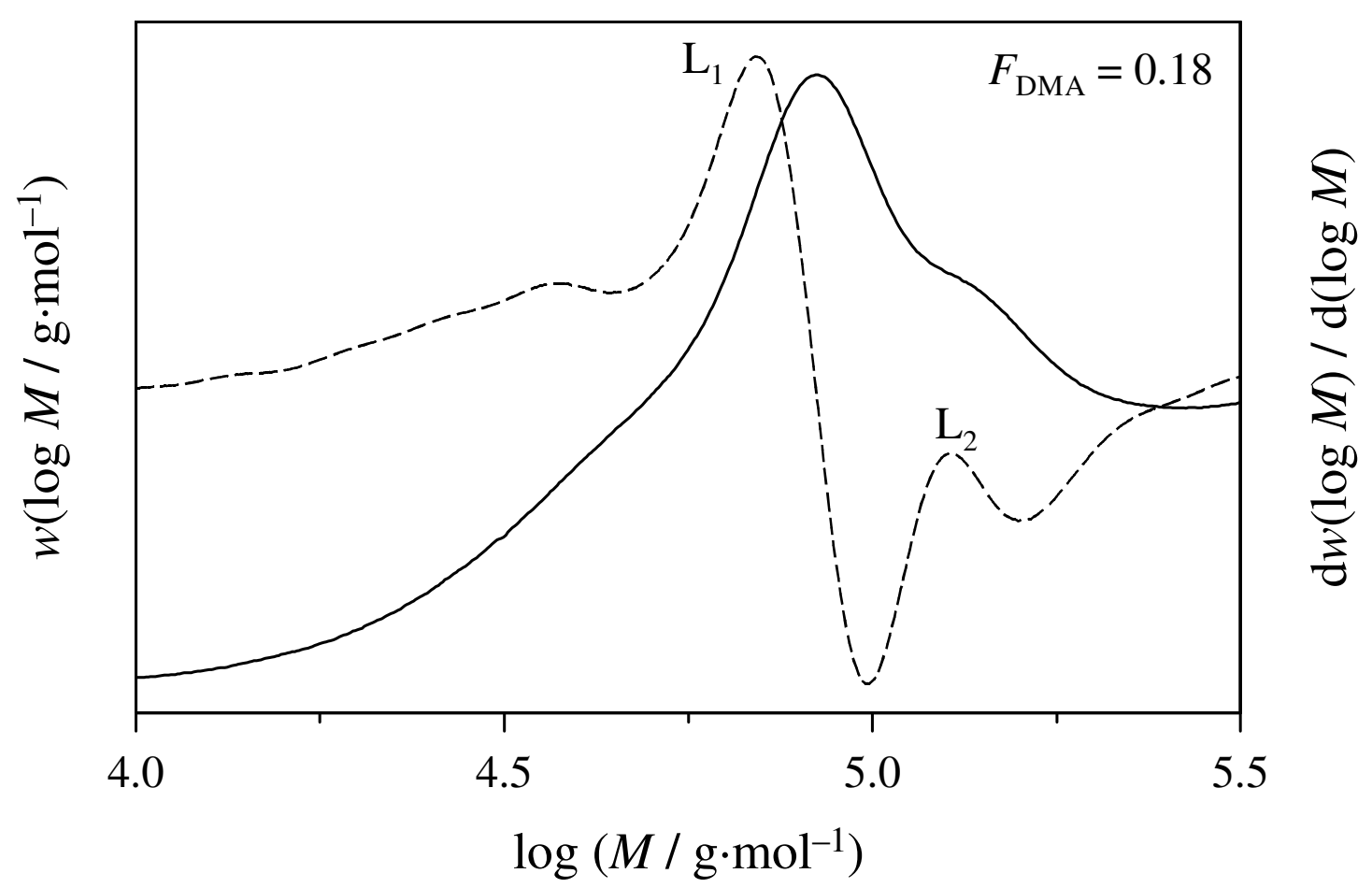

Fig. 5.9 Experimental molecular weight distribution (full line) of a DA/DMA copolymer $\left(f_{\text {DMA }}=0.06\right)$ produced at $40{ }^{\circ} \mathrm{C}$ and 1000 bar at $100 \mathrm{~Hz}$. The associated first derivative curve is given by a dashed line. 
The data set is calibrated with the Mark-Houwink coefficients from reference [29] according to Eq. 3.17. The individual data points have been connected by a spline function and the Mark-Houwink parameters corresponding to intermediate compositions are calculated by linear interpolation between two measured data points. The resulting $k_{\mathrm{p}, \text { copo }}$ data set obtained at $23{ }^{\circ} \mathrm{C} / 1000$ bar and $40{ }^{\circ} \mathrm{C} / 1000$ bar is shown in Fig. 5.10 and Fig. 5.11 respectively.

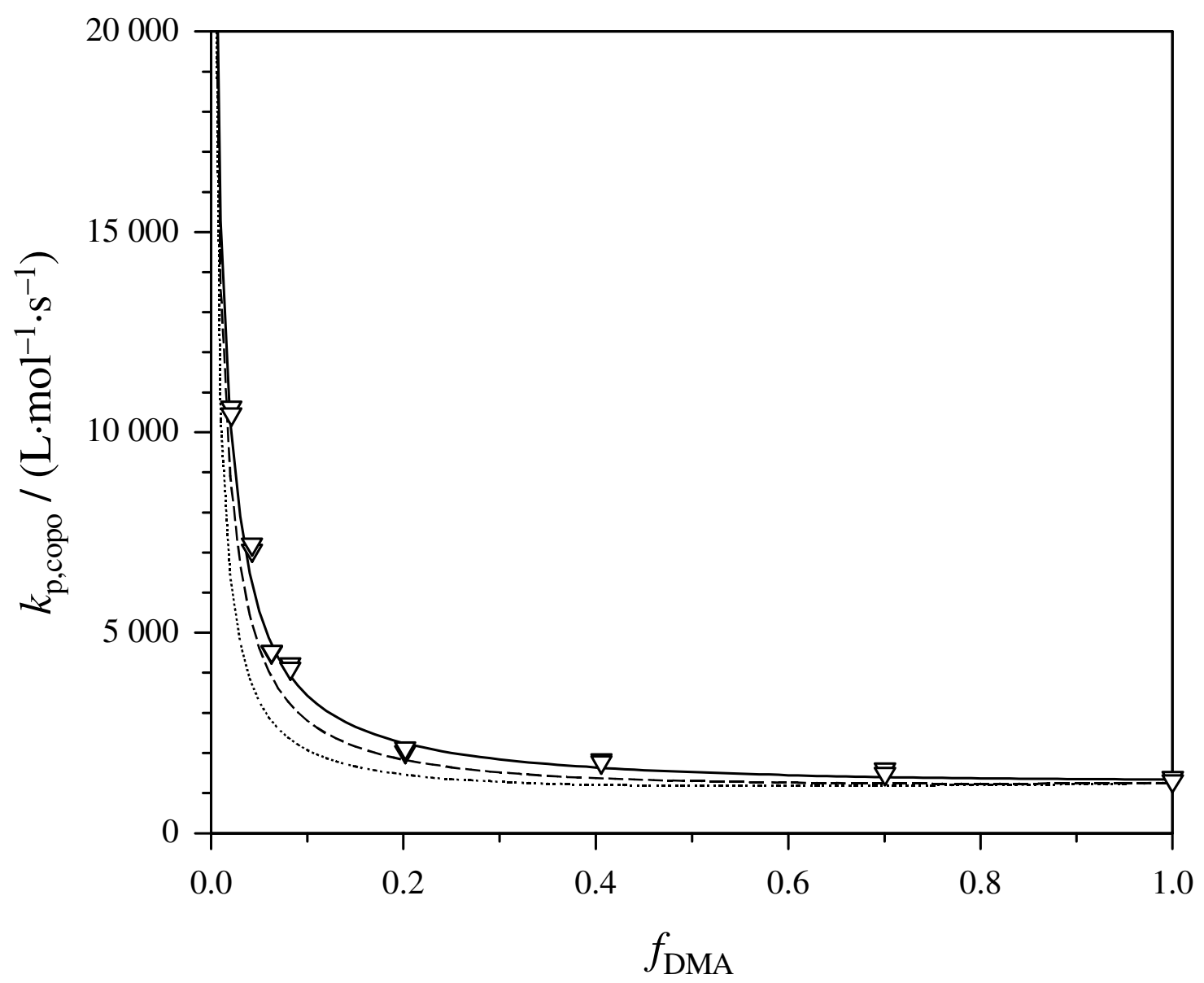

Fig. 5.10 Copolymerization propagation rate coefficients determined by PLP-SEC for the DA/DMA system as a function of the mole fraction of DMA in the monomer mixture $\left(f_{\text {DMA }}\right)$ at $23{ }^{\circ} \mathrm{C}$ and 1000 bar. The full line corresponds to a fit according to the IPUE model as described in the text. The dotted line is a terminal model representation with the reactivity ratio data $r_{\mathrm{mac}}=2.55$ and $r_{\mathrm{ac}}=0.29$ taken from the Lewis-Mayo fit to the overall composition data (Fig. 5.3). The dashed line represents the terminal model fit to the composition data with reactivity ratios $r_{\text {mac }}=2.21$ and $r_{\mathrm{ac}}=0.38$ (Tab. 5.1). 


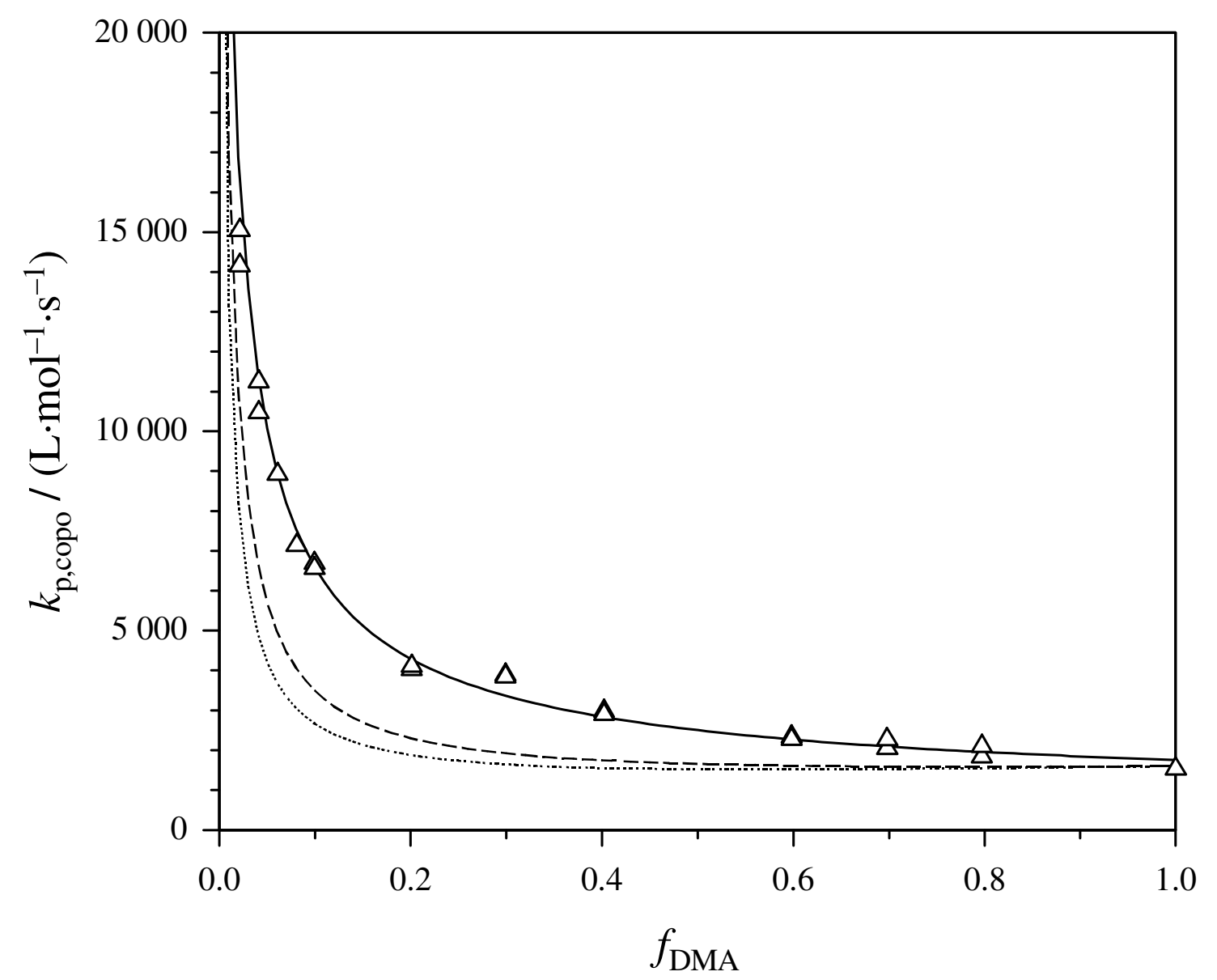

Fig. 5.11 Copolymerization propagation rate coefficients determined by PLP-SEC for the DA/DMA system as a function of the mole fraction of DMA in the monomer mixture $\left(f_{\mathrm{DMA}}\right)$ at $40{ }^{\circ} \mathrm{C}$ and 1000 bar. The full line corresponds to a fit according to the IPUE model as described in the text. The dotted line is a terminal model representation with the reactivity ratio data $r_{\text {mac }}=2.55$ and $r_{\mathrm{ac}}=0.29$ taken from the Lewis-Mayo fit to the overall composition data (Fig. 5.3). The dashed line represents the terminal model fit to the composition data with reactivity ratios $r_{\mathrm{mac}}=2.21$ and $r_{\mathrm{ac}}=0.38$ (Tab. 5.1).

The experimental conditions and all results for the DA/DMA system obtained by PLP-SEC are summarized in Tab. 5.5 (at $23{ }^{\circ} \mathrm{C}$ and 1000 bar) and in Tab. 5.6 (at $40{ }^{\circ} \mathrm{C}$ and 1000 bar). 


\begin{tabular}{ccccc}
\hline$f^{0}{ }_{\mathrm{DMA}}$ & $v / \mathrm{Hz}$ & $M_{\mathrm{L} 1} / \mathrm{g} \mathrm{mol}^{-1}$ & $M_{\mathrm{L} 2} / \mathrm{g} \mathrm{mol}^{-1}$ & $k_{\mathrm{p}} / \mathrm{L} \mathrm{mol}^{-1} \mathrm{~s}^{-1}$ \\
\hline 1.00 & 100 & 7693 & 15438 & 1309 \\
1.00 & 100 & 7237 & 13597 & 1233 \\
0.70 & 100 & 9014 & 17334 & 1415 \\
0.70 & 100 & 9691 & 18975 & 1522 \\
0.40 & 100 & 11520 & 23631 & 1698 \\
0.40 & 100 & 11872 & 23941 & 1751 \\
0.20 & 100 & 19712 & 35620 & 1958 \\
0.20 & 100 & 20668 & 36591 & 2049 \\
0.08 & 100 & 35999 & 71504 & 4140 \\
0.08 & 100 & 35092 & 69501 & 4038 \\
0.06 & 100 & 40570 & 82543 & 4463 \\
0.06 & 100 & 40398 & 82871 & 4444 \\
0.04 & 100 & 60563 & 105039 & 6984 \\
0.04 & 100 & 61844 & 104393 & 7135 \\
0.02 & 100 & 79779 & 146423 & 10552 \\
0.02 & 100 & 78571 & 141120 & 10384 \\
0.00 & 100 & 181526 & 335336 & 31395 \\
0.00 & 100 & 186728 & 345001 & 32370 \\
\hline
\end{tabular}

Tab. 5.5 PLP-SEC results for the copolymerization of DA/DMA at $23{ }^{\circ} \mathrm{C}$ and $1000 \mathrm{bar}$.

\begin{tabular}{ccccc}
\hline$f^{0}{ }_{\mathrm{DMA}}$ & $v / \mathrm{Hz}$ & $M_{\mathrm{L} 1} / \mathrm{g} \mathrm{mol}^{-1}$ & $M_{\mathrm{L} 2} / \mathrm{g} \mathrm{mol}^{-1}$ & $k_{\mathrm{p}} / \mathrm{L} \mathrm{mol}^{-1} \mathrm{~s}^{-1}$ \\
\hline 0.10 & 100 & 44195 & 90174 & 6630 \\
0.10 & 100 & 45113 & 93207 & 6763 \\
0.08 & 100 & 53604 & 99598 & 7215 \\
0.06 & 100 & 68969 & 127956 & 8994 \\
0.04 & 100 & 78759 & 171120 & 10546 \\
0.04 & 100 & 84567 & 166188 & 11329 \\
0.02 & 100 & 87438 & 249517 & 14238 \\
0.02 & 100 & 92427 & 235440 & 15119 \\
\hline
\end{tabular}

Tab. 5.6 PLP-SEC results for the copolymerization of DA/DMA at $f_{\mathrm{DA}}>0.9$ at $40{ }^{\circ} \mathrm{C}$ and 1000 bar.

The $k_{\mathrm{p}, \text { copo }}$ values for both cases are fitted by two procedures: (a) via the terminal model expression (Eq. 5.2) and (b) via the IPUE effect model by simultaneously fitting the $k_{\mathrm{p}, \mathrm{copo}}$ and composition data. The data analysis by the IPUE effect model was carried out by the procedure outlined in section 5.1.1.

The dashed lines in Figs. 5.10 and 5.11 show the fit of the terminal model to the $k_{\text {p,copo }}$ data, as calculated by Eq. 5.2 using the $r_{\mathrm{mac}}$ and $r_{\mathrm{ac}}$ estimates from the fit to the polymer composition (Tab. 5.1), the dotted lines represent a terminal model fit with reactivity ratios taken from a 
Lewis-Mayo fit to the overall composition data (Fig. 5.3). The terminal model adequately describes the data of the DA/DMA copolymerization at $23{ }^{\circ} \mathrm{C}$ and $1000 \mathrm{bar}$, but in case of the DA/DMA copolymerization at $40{ }^{\circ} \mathrm{C}$ and 1000 bar it is clear that the terminal model does not fit both the composition and $k_{\mathrm{p} \text {,copo }}$ data simultaneously.

Because of the failure of the terminal model for DA/DMA at $40{ }^{\circ} \mathrm{C}$ and 1000 bar, the IPUE model was examined. All parameters of this model were fitted to the combined composition and $k_{\mathrm{p}, \mathrm{copo}}$ data set. Tab. 5.11 contains a summary of the parameter estimates.

\subsubsection{METHYL ACRYLATE / DODECYL METHACRYLATE}

In order to obtain well structured MWDs at very high initial mole fraction of MA in the monomer mixture (that is at $f_{\mathrm{MA}}>0.94$ ), the initiator DMPA was used at initial concentrations close to $2 \cdot 10^{-3} \mathrm{~mol} \cdot \mathrm{L}^{-1}$. This concentration is 2.5 times lower as the one that is normally used in PLP-SEC experiments. Nicely structured molecular weight distributions are obtained in all cases. Fig. 5.12 shows the first derivatives of the MWD of a MA/DMA copolymer produced at $22{ }^{\circ} \mathrm{C} / 1000$ bar at $100 \mathrm{~Hz}$. Fig. 5.13 presents the MWD and the associated first derivative curve of a MA/DMA copolymer obtained at $40{ }^{\circ} \mathrm{C}$ and 1000 bar by pulsed laser polymerization at a pulse repetition rate of $100 \mathrm{~Hz}$.

The MWDs for MA/DMA copolymerization at $40{ }^{\circ} \mathrm{C}$ and 1000 bar have partly been measured by Buback et al. [29]. Additionally ten values of $k_{\mathrm{p}, \text { copo }}$ at $f_{\mathrm{MA}}>0.9$ were added to the data of the MA/DMA copolymerization at $40{ }^{\circ} \mathrm{C}$ and 1000 bar. The results for the MA/DMA system concerning Mark-Houwink parameter determination and evaluation have already been described [29]. Propagation rate coefficients for the system MA/DMA measured at $22{ }^{\circ} \mathrm{C}$ and 1000 bar will be presented. 


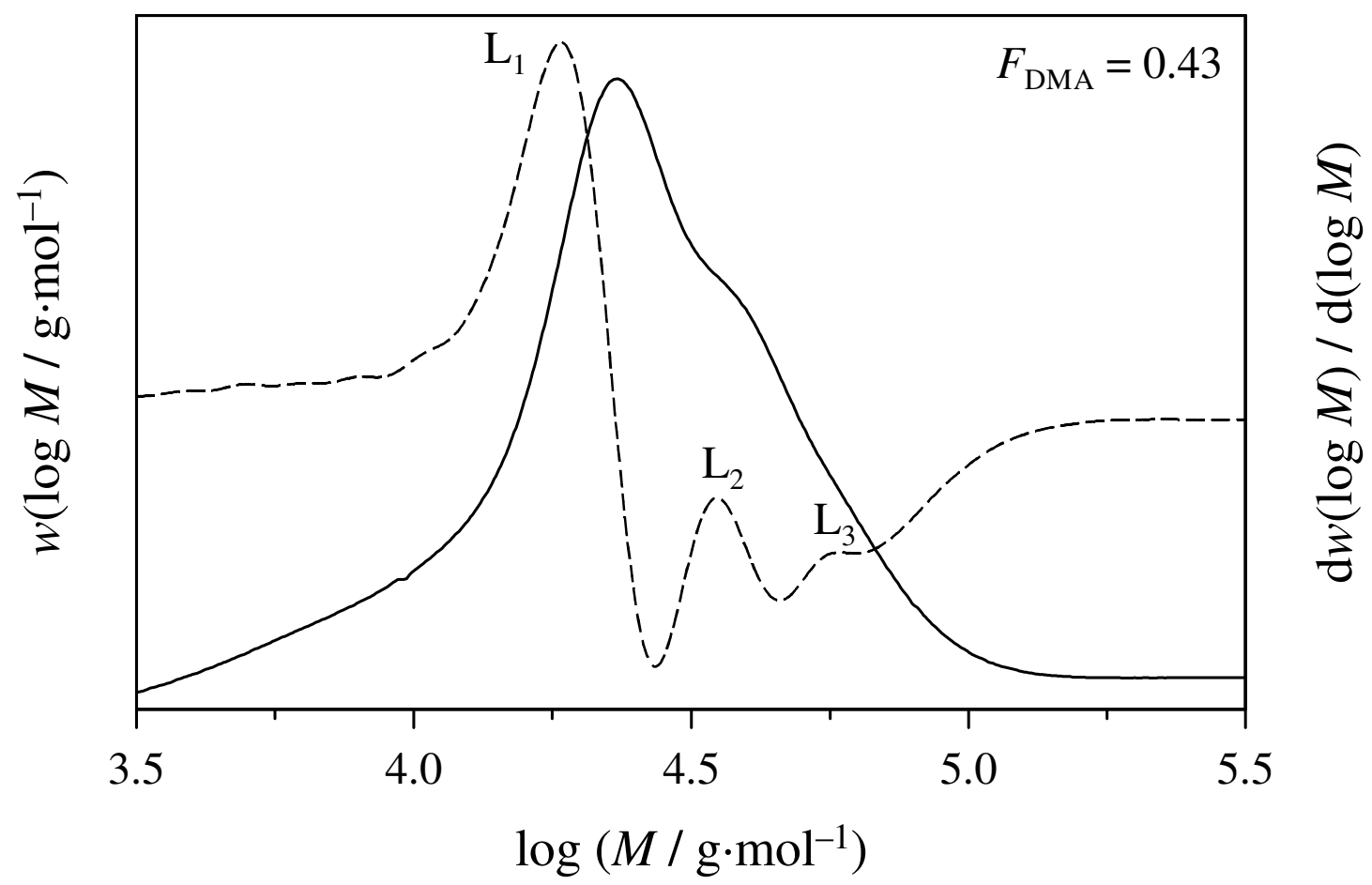

Fig. 5.12 Experimental MWD (full line) of a MA/DMA copolymer $\left(f_{\mathrm{DMA}}=0.20\right)$ produced at $22{ }^{\circ} \mathrm{C}$ and 1000 bar at $100 \mathrm{~Hz}$. A dashed line gives the associated first derivative curve.

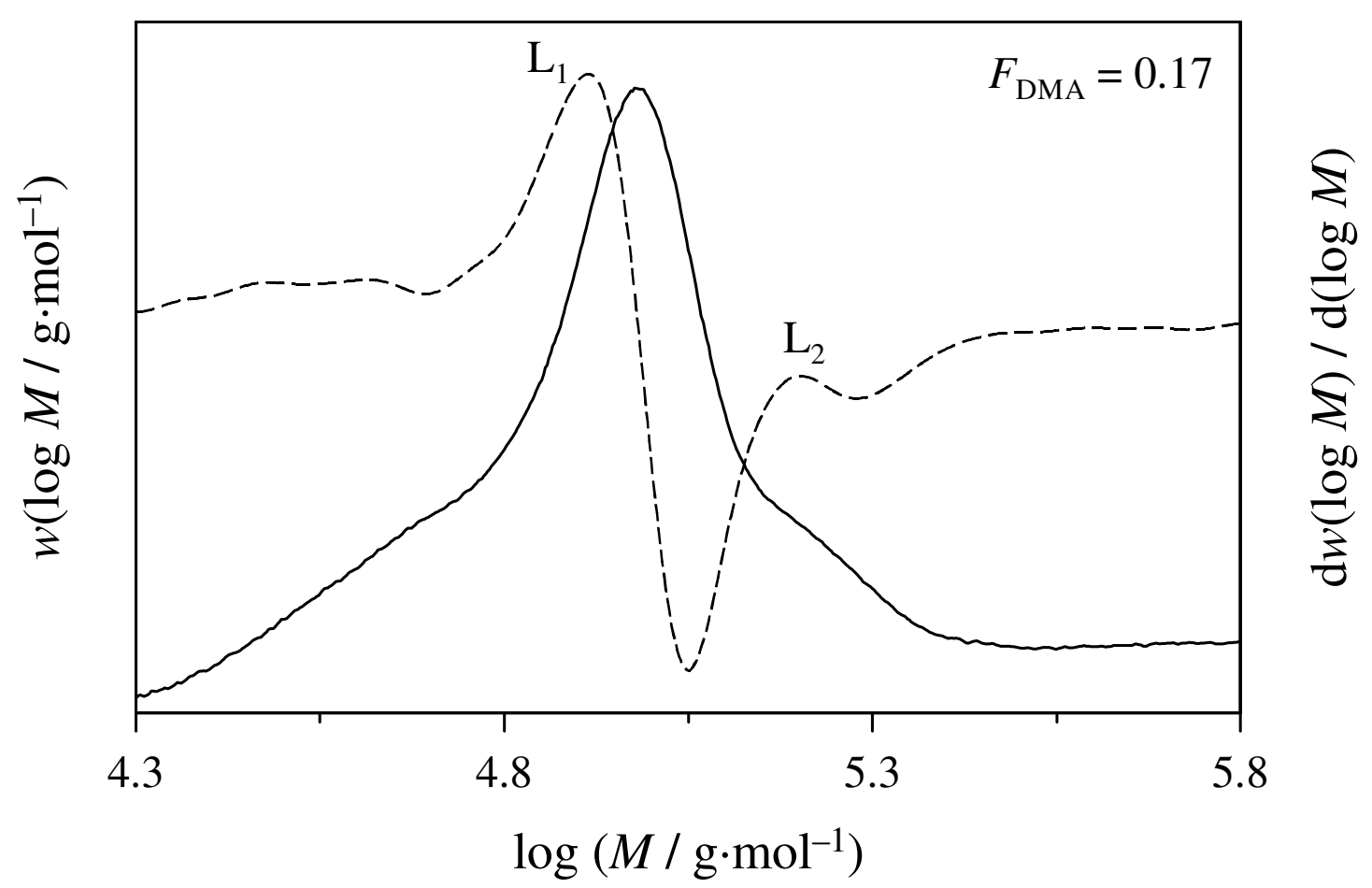

Fig. 5.13 Experimental molecular weight distribution (full line) of a MA/DMA copolymer $\left(f_{\text {DMA }}=0.06\right)$ produced at $40{ }^{\circ} \mathrm{C}$ and 1000 bar at $100 \mathrm{~Hz}$. The associated first derivative curve is given by a dashed line. 
For SEC calibration, the Mark-Houwink parameters for several copolymer compositions have been connected by a spline function and the Mark-Houwink parameters corresponding to intermediate compositions are calculated by linear interpolation between two measured data points. The resulting $k_{\mathrm{p} \text {,copo }}$ data set obtained at $22{ }^{\circ} \mathrm{C} / 1000$ bar and $40{ }^{\circ} \mathrm{C} / 1000$ bar is shown in Fig. 5.14 and Fig. 5.15 respectively.

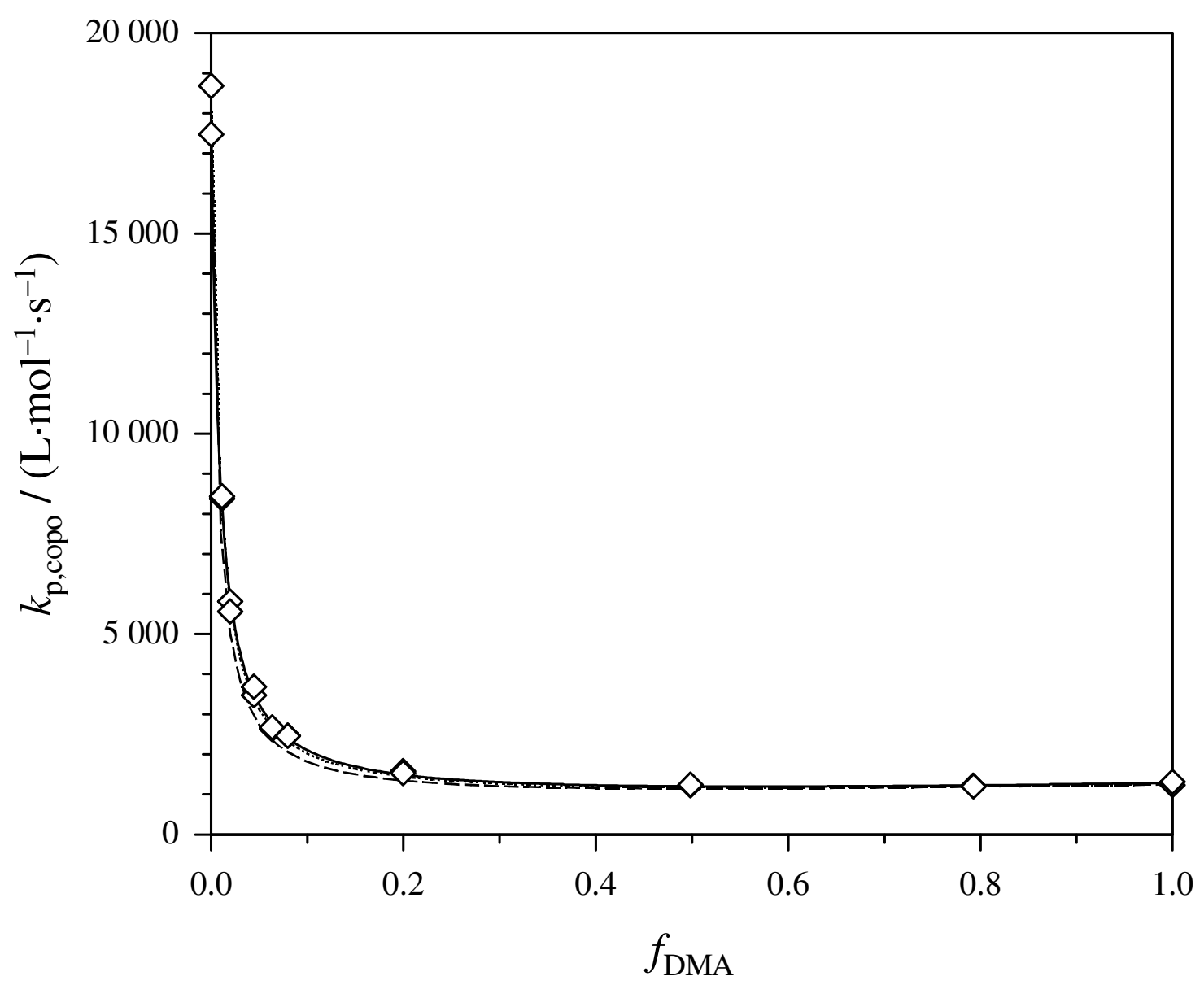

Fig. 5.14 Copolymerization propagation rate coefficients determined by PLP-SEC for the MA/DMA system as a function of the mole fraction of DMA in the monomer mixture $\left(f_{\mathrm{DMA}}\right)$ at $22{ }^{\circ} \mathrm{C}$ and 1000 bar. The full line corresponds to a fit according to the IPUE model as described in the text. The dotted line is a terminal model representation with the reactivity ratio data $r_{\mathrm{mac}}=2.55$ and $r_{\mathrm{ac}}=0.29$ taken from the Lewis-Mayo fit to the overall composition data (Fig. 5.3). The dashed line represents the terminal model fit to the composition data with reactivity ratios $r_{\text {mac }}=2.66$ and $r_{\mathrm{ac}}=0.25$ (Tab. 5.1). 


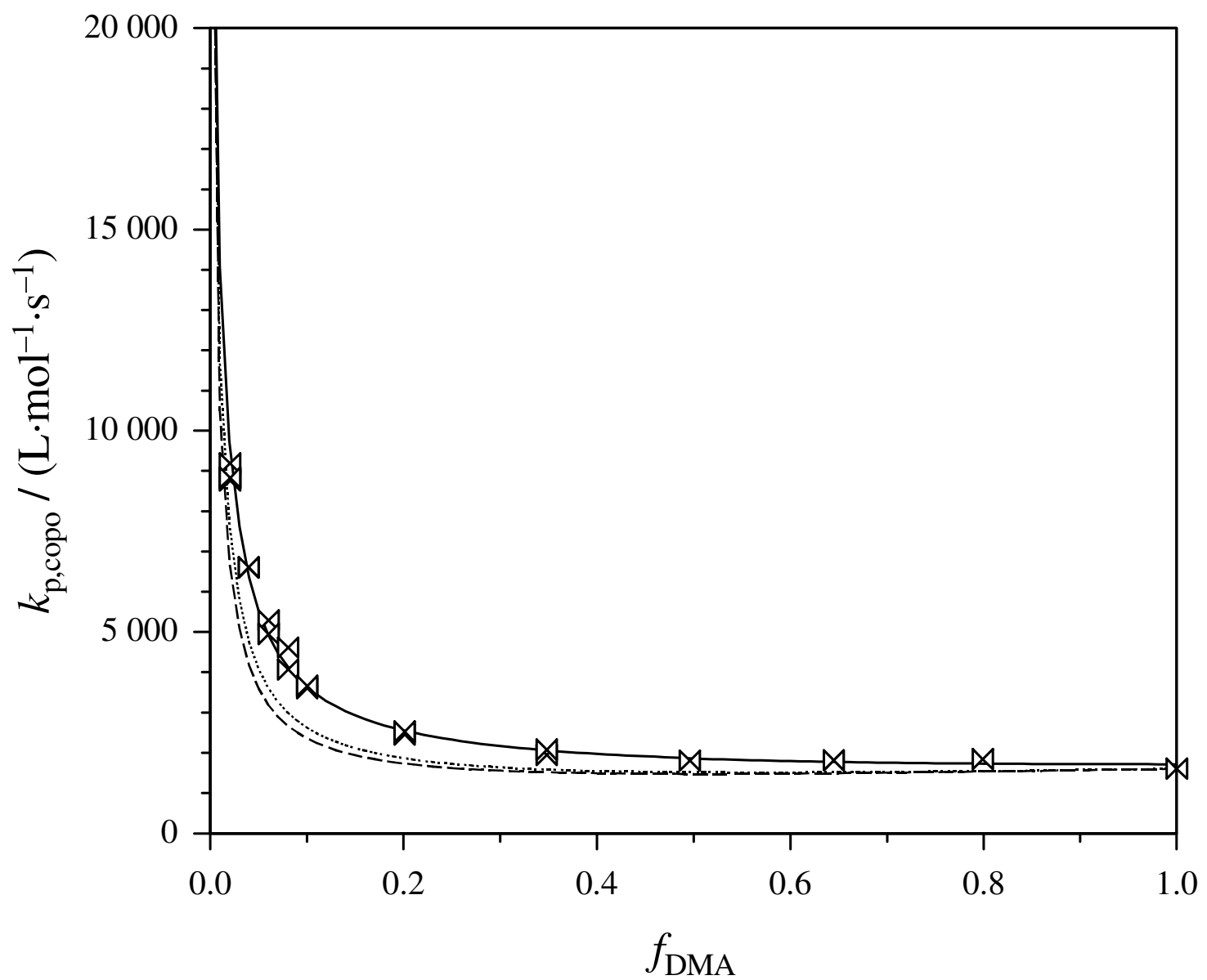

Fig. 5.15 Copolymerization propagation rate coefficients determined by PLP-SEC for the MA/DMA system as a function of the mole fraction of DMA in the monomer mixture $\left(f_{\mathrm{DMA}}\right)$ at $40{ }^{\circ} \mathrm{C}$ and 1000 bar. The full line corresponds to a fit according to the IPUE model as described in the text. The dotted line is a terminal model representation with the reactivity ratio data $r_{\mathrm{mac}}=2.55$ and $r_{\mathrm{ac}}=0.29$ taken from the Lewis-Mayo fit to the overall composition data (Fig. 5.3). The dashed line represents the terminal model fit to the composition data with reactivity ratios $r_{\text {mac }}=2.66$ and $r_{\mathrm{ac}}=0.25$ (Tab. 5.1).

The resulting propagation rate coefficients for the MA/DMA system are given in Tab. 5.7 (at $22{ }^{\circ} \mathrm{C}$ and 1000 bar) and in Tab. 5.8 (at $40{ }^{\circ} \mathrm{C}$ and 1000 bar). 


\begin{tabular}{ccccc}
\hline$f^{0}{ }_{\mathrm{MMA}}$ & $v / \mathrm{Hz}$ & $M_{\mathrm{L} 1} / \mathrm{g} \mathrm{mol}^{-1}$ & $M_{\mathrm{L} 2} / \mathrm{g} \mathrm{mol}^{-1}$ & $k_{\mathrm{p}} / \mathrm{L} \mathrm{mol}^{-1} \mathrm{~s}^{-1}$ \\
\hline 1.00 & 100 & 7693 & 15438 & 1309 \\
1.00 & 100 & 7237 & 13597 & 1233 \\
0.79 & 100 & 8872 & 16642 & 1220 \\
0.79 & 100 & 8738 & 16834 & 1202 \\
0.50 & 100 & 11429 & 23195 & 1228 \\
0.50 & 100 & 11556 & 22725 & 1242 \\
0.20 & 100 & 24632 & 47874 & 1581 \\
0.20 & 100 & 23895 & 45415 & 1534 \\
0.08 & 100 & 41937 & 88634 & 2464 \\
0.08 & 100 & 41802 & 86377 & 2456 \\
0.06 & 100 & 44671 & 91768 & 2625 \\
0.06 & 100 & 45459 & 93629 & 2674 \\
0.04 & 100 & 56711 & 106094 & 3471 \\
0.04 & 100 & 59961 & 114251 & 3685 \\
0.02 & 100 & 90091 & 175671 & 5809 \\
0.02 & 100 & 86469 & 176311 & 5561 \\
0.01 & 100 & 123453 & 257810 & 8377 \\
0.01 & 100 & 124366 & 263512 & 8442 \\
0.00 & 100 & 181853 & 338057 & 17476 \\
0.00 & 100 & 194258 & 340463 & 18683 \\
\hline
\end{tabular}

Tab. 5.7 PLP-SEC results for the copolymerization of MA/DMA at $22{ }^{\circ} \mathrm{C}$ and $1000 \mathrm{bar}$.

\begin{tabular}{ccccc}
\hline$f^{0}{ }_{\text {DMA }}$ & $v / \mathrm{Hz}$ & $M_{\mathrm{L} 1} / \mathrm{g} \mathrm{mol}^{-1}$ & $M_{\mathrm{L} 2} / \mathrm{g} \mathrm{mol}^{-1}$ & $k_{\mathrm{p}} / \mathrm{L} \mathrm{mol}^{-1} \mathrm{~s}^{-1}$ \\
\hline 0.10 & 100 & 58332 & 108375 & 3604 \\
0.10 & 100 & 59231 & 109964 & 3662 \\
0.08 & 100 & 67543 & 131063 & 4069 \\
0.08 & 100 & 76075 & 152423 & 4611 \\
0.06 & 100 & 81051 & 158314 & 4950 \\
0.06 & 100 & 86289 & 158198 & 5290 \\
0.04 & 100 & 102014 & 211836 & 6598 \\
0.04 & 100 & 102092 & 207922 & 6603 \\
0.02 & 100 & 131229 & 277000 & 8813 \\
0.02 & 100 & 136439 & 282085 & 9187 \\
0.02 & 100 & 130407 & 278356 & 8829 \\
0.02 & 100 & 131392 & 264028 & 8829 \\
\hline
\end{tabular}

Tab. 5.8 PLP-SEC results for the copolymerization of MA/DMA at $40{ }^{\circ} \mathrm{C}$ and $1000 \mathrm{bar}$.

The obtained propagation rate coefficients, given in Tab. 5.7 and 5.8, have been analyzed by the terminal and IPUE models. For both sets of polymerization conditions, the simultaneous fitting of composition and $k_{\mathrm{p} \text {,copo }}$ has been carried out according to the procedure outlined in section 5.1.1. 
Additionally to the terminal model, the IPUE model was examined. Fig. 5.14 and 5.15 show the results of fitting the terminal model to the $k_{\mathrm{p}, \text { copo }}$ data and the IPUE model to the combined composition and $k_{\text {p,copo }}$ data for the system MA/DMA at $22^{\circ} \mathrm{C} / 1000$ bar and $40{ }^{\circ} \mathrm{C} / 1000$ bar. At lower temperature $\left(22^{\circ} \mathrm{C}\right)$ the system MA/DMA can be well represented by the terminal model, but Fig. 5.15 illustrates its inadequacy in describing the MA/DMA system at $40{ }^{\circ} \mathrm{C}$.

Tab. 5.11 contains a summary of the parameter estimates.

\subsubsection{DODECYL ACRYLATE / METHYL METHACRYLATE}

The system DA/MMA at $40{ }^{\circ} \mathrm{C}$ and 1000 bar has already been studied by Buback et al. [29]. The resulting $k_{\mathrm{p}, \mathrm{copo}}$ data were analyzed with respect to the terminal, implicit and explicit penultimate unit models. The authors showed the failure of the terminal model to fit both the composition and $k_{\mathrm{p} \text {,copo }}$ data simultaneously. Thus, the next case considered was the IPUE fitting. It turned out that also this model was not adequate in describing the system at high mole fraction of methyl methacrylate. The EPUE model has also been used for simultaneous nonlinear fitting of the $k_{\mathrm{p} \text {,copo }}$ and composition data. It was found that the agreement between measured and modeled data is not perfect at high $f_{\text {mac }}$ even with the EPUE model.

The present work focuses on the propagation step of DA/MMA copolymerization at $23{ }^{\circ} \mathrm{C}$ and 1000 bar. It was the enormous advantage that existing Mark-Houwink parameters determined by the same authors [29] can be used.

In order to obtain well pronounced MWDs at very high initial mole fraction of DA in the monomer mixture (that is at $f_{\mathrm{DA}}>0.94$ ) the initiator DMPA was used at initial concentrations close to $2 \cdot 10^{-3} \mathrm{~mol} \cdot \mathrm{L}^{-1}$. This concentration is 2.5 times below the one normally used in the previous studies [29]. Nicely structured molecular weight distributions are obtained in all cases. Fig. 5.16 shows the first derivatives of the MWD of a DA/MMA copolymer produced at $23{ }^{\circ} \mathrm{C} / 1000$ bar at $100 \mathrm{~Hz}$. 


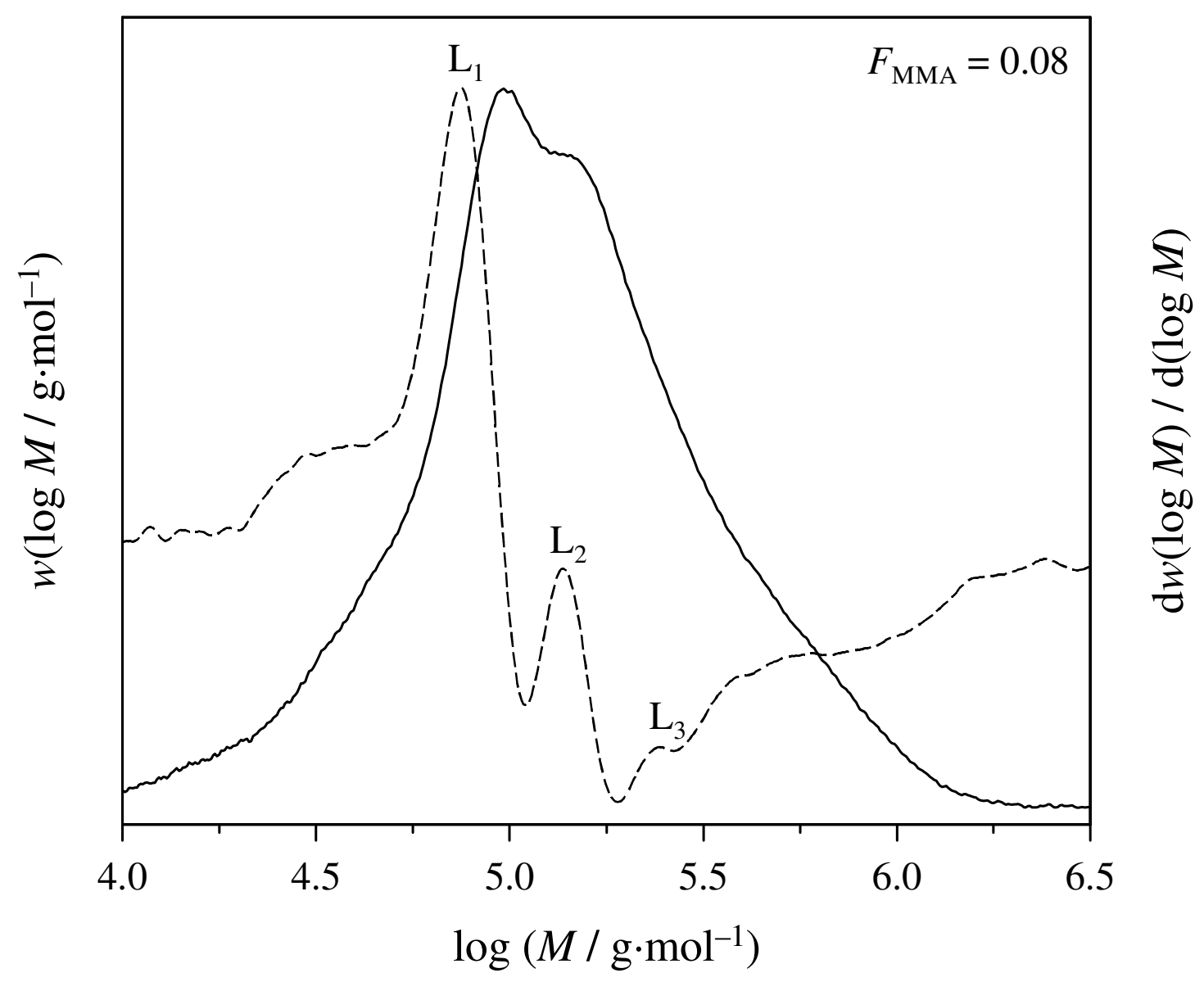

Fig. 5.16 Experimental molecular weight distribution (full line) of a DA/MMA copolymer $\left(f_{\text {MMA }}=0.02\right)$ produced at $23{ }^{\circ} \mathrm{C}$ and 1000 bar at $100 \mathrm{~Hz}$ with associated first derivative curve (dashed line).

In order to obtain SEC calibration the individual Mark-Houwink parameters have been connected by a spline function. The Mark-Houwink parameters corresponding to intermediate compositions are calculated by linear interpolation between two measured data points. The resulting $k_{\mathrm{p}, \text { copo }}$ data set obtained at $23{ }^{\circ} \mathrm{C} / 1000$ bar is shown in Fig. 5.17. 


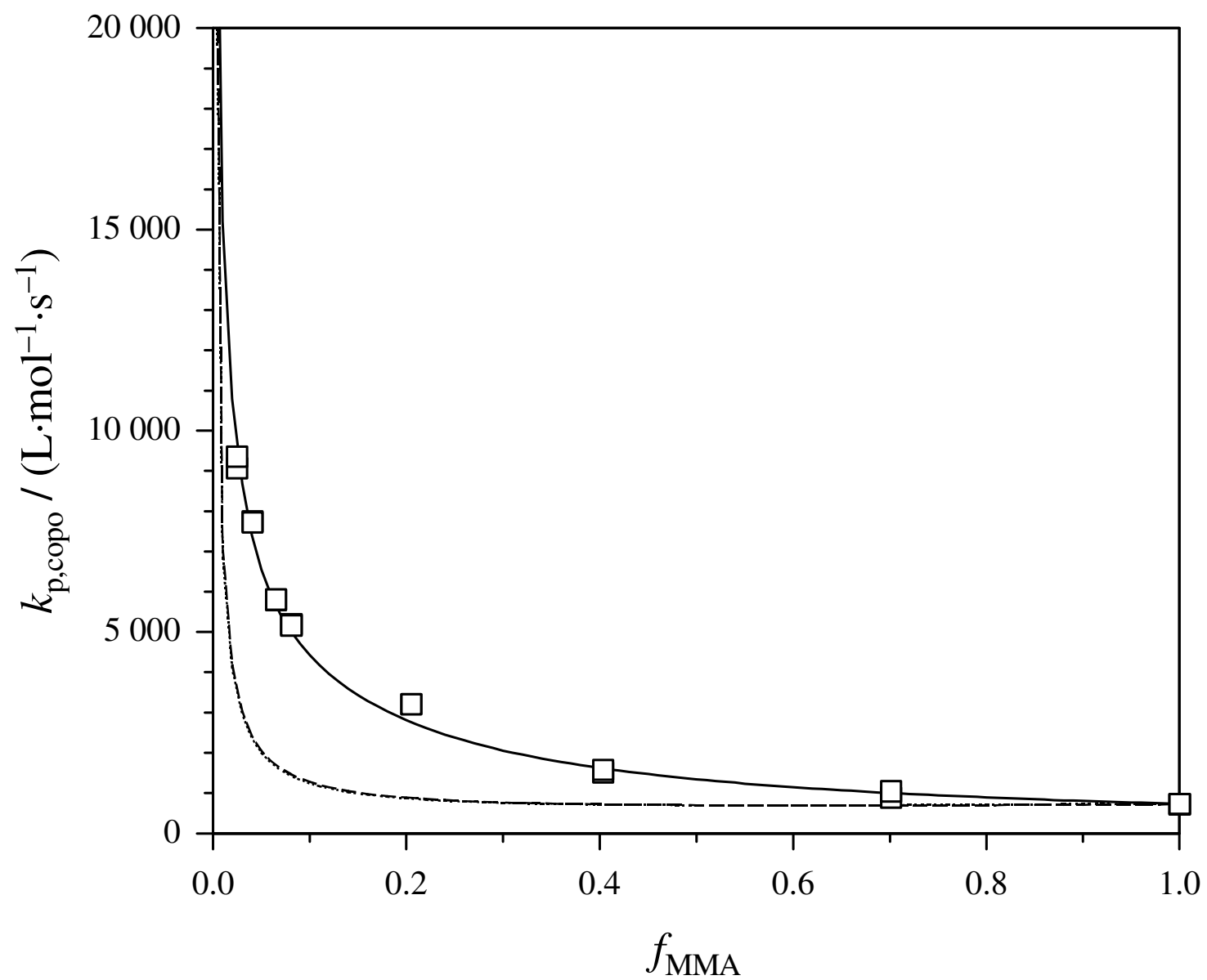

Fig. 5.17 Copolymerization propagation rate coefficients determined by PLP-SEC for the DA/MMA system as a function of the mole fraction of MMA in the monomer mixture $\left(f_{\mathrm{MMA}}\right)$ at $23{ }^{\circ} \mathrm{C}$ and 1000 bar. The full line corresponds to a fit according to the IPUE model as described in the text. The dotted line is a terminal model representation with the reactivity ratio data $r_{\mathrm{mac}}=2.55$ and $r_{\mathrm{ac}}=0.29$ taken from the Lewis-Mayo fit to the overall composition data (Fig. 5.3). The dashed line represents the terminal model fit to the composition data with reactivity ratios $r_{\text {mac }}=2.42$ and $r_{\mathrm{ac}}=0.29$ (Tab. 5.1).

The parameters of the PLP-SEC experiments and the resulting $k_{\mathrm{p}}$ values for the entire data set (together with the pulse frequency and the inflection points used for $k_{\mathrm{p}, \text { copo }}$ determination) are given in Tab. 5.9. 


\begin{tabular}{ccccc}
\hline$f^{0}{ }_{\mathrm{MMA}}$ & $v / \mathrm{Hz}$ & $M_{\mathrm{L} 1} / \mathrm{g} \mathrm{mol}^{-1}$ & $M_{\mathrm{L} 2} / \mathrm{g} \mathrm{mol}^{-1}$ & $k_{\mathrm{p}} / \mathrm{L} \mathrm{mol}^{-1} \mathrm{~s}^{-1}$ \\
\hline 1.00 & 40 & 16853 & 32228 & 715 \\
1.00 & 40 & 17225 & 33007 & 731 \\
0.70 & 100 & 6758 & 14256 & 885 \\
0.40 & 100 & 10545 & 22610 & 1577 \\
0.40 & 100 & 10107 & 22405 & 1514 \\
0.21 & 100 & 18420 & 33647 & 3202 \\
0.21 & 100 & 18459 & 34796 & 3209 \\
0.08 & 100 & 32391 & 64908 & 5161 \\
0.08 & 100 & 32598 & 66177 & 5193 \\
0.07 & 100 & 36933 & 75395 & 5808 \\
0.07 & 100 & 36867 & 76227 & 5798 \\
0.04 & 100 & 60483 & 94813 & 7722 \\
0.04 & 100 & 60661 & 93325 & 7745 \\
0.02 & 100 & 72196 & 127617 & 9065 \\
0.02 & 100 & 74425 & 136408 & 9352 \\
0.00 & 100 & 181526 & 335336 & 31395 \\
0.00 & 100 & 186728 & 345001 & 32370 \\
\hline
\end{tabular}

Tab. 5.9 PLP-SEC results for the copolymerization of DA/MMA at $23{ }^{\circ} \mathrm{C}$ and $1000 \mathrm{bar}$.

The resulting data set was analyzed by the terminal model first. It was found that it is impossible to fit composition and rate data by the terminal model with the same set of reactivity ratios. Therefore a data analysis by the implicit IPUE model was carried out by the procedure outlined in section 5.1.1. It turned out that the IPUE model cannot yield reasonable radical reactivity ratios for the system DA/MMA at $23{ }^{\circ} \mathrm{C} / 1000$ bar (Tab. 5.11).

\subsection{DISCUSSION}

It is worth mentioning that it is rather difficult to apply any copolymerization model to an acrylate/methacrylate system measured at temperatures above $30{ }^{\circ} \mathrm{C}$. Dating back to the early study of Davis et al. [16], it was evident that the required PLP-SEC consistency checks were difficult to fulfill for acrylate monomers. Rather than producing well structured distributions, the PLP-generated polyacrylate MWDs were broader and indistinct. Further studies with butyl acrylate [37,41] demonstrated that $k_{\mathrm{p}}$ estimates could only be obtained by carrying out experiments at temperatures below $30^{\circ} \mathrm{C}$ and at high laser repetition rate (e.g., 50 or $100 \mathrm{~Hz}$ ). The reasons for the difficulty in obtaining $k_{\mathrm{p}}$ at higher temperatures remained a matter of speculation. Various hypotheses have been put forward (see van Herk [1] for an overview) which included high rates of transfer to monomer/solvent, significant exotherms during laser 
illumination, intermolecular chain transfer to polymer confounding accurate MWD analysis by SEC, and intramolecular transfer (backbiting) [38,39,40,41,42,43] that can disrupt the relationship between chain length and radical life time expressed by Eq.3.12. Recent experimental and modeling studies have shown that the intramolecular transfer event is the most significant effect in acrylate chain-growth kinetics. There is strong evidence that monomer addition to the mid-chain radical (resulting from backbiting) proceeds at a much slower rate than addition to the parent terminal radical. After the first monomer addition, the propagation radical assumes secondary chain-end character and continues to grow with the chain-end $k_{\mathrm{p}}$ value. It has been determined that propagation rates of methyl acrylate dimer [44], butyl acrylate dimer [45] and dodecyl acrylate dimer (section 8) (species that form radicals of a structure similar to the one of mid-chain radicals) are by at least two orders of magnitude lower than of acrylate chain-end radicals. Thus, if the fraction of chains that undergo backbiting is sufficiently large, the linear relationship between chain length and radical lifetime given by Eq. 3.12 is disturbed and no PLP structure will develop. This conclusion is supported by recent simulation studies by Arzamendy et al. [8] and Nikitin et al. [46].

Another difficulty in the determination of copolymerization parameters for acrylate/methacrylate systems is the large difference between $k_{\mathrm{p}}$ values for acrylates and methacrylates (e.g., $1.44 \cdot 10^{4} \mathrm{~L} \cdot \mathrm{mol}^{-1} \cdot \mathrm{s}^{-1}$ for butyl acrylate at $20^{\circ} \mathrm{C}$ [2,37] compared with $314 \mathrm{~L} \cdot \mathrm{mol}^{-1} \cdot \mathrm{s}^{-1}$ for butyl methacrylate [47]). This difference results in $r_{\mathrm{ac}} / k_{\mathrm{p}, \mathrm{ac}} \ll r_{\mathrm{mac}} / k_{\mathrm{p}, \mathrm{mac}}$ that, according to Eq. 5.2, may cause an insensitivity of the IPUE fitting procedure for the $s_{\mathrm{ac}}$ value. In Tab. $5.10 \frac{r_{1} \cdot f_{1}}{k_{\mathrm{p} 11}}$ and $\frac{r_{2} \cdot f_{2}}{k_{\mathrm{p} 22}}$ from Eq. 5.2 for the system DA/MMA measured at $23{ }^{\circ} \mathrm{C} / 1000$ bar are compared. Index 1 corresponds to MMA and index 2 to DA $\left(r_{1}=2.55\right.$ and $\left.r_{2}=0.29\right) . \frac{r_{2} \cdot f_{2}}{k_{\mathrm{p} 22}}<<\frac{r_{1} \cdot f_{1}}{k_{\mathrm{p} 11}}$ within the entire $f_{1}$ range covered in Tab. 5.10. This observation allows for the modification of Eq. 5.2 as follows:

$$
k_{\mathrm{p}, \mathrm{copo}}=\frac{r_{1} \cdot f_{1}^{2}+r_{2} \cdot f_{2}^{2}+2 \cdot f_{1} \cdot f_{2}}{r_{1} \cdot f_{1} / k_{\mathrm{p} 11}}
$$




\begin{tabular}{|c|c|c|c|}
\hline$f_{1}$ & $\left(r_{1} \cdot f_{1}\right) / k_{\mathrm{p} 11}$ & $\left(r_{2} \cdot f_{2}\right) / k_{\mathrm{p} 22}$ & {$\left[\left(r_{2} \cdot f_{2}\right) / k_{\mathrm{p} 22}\right] /\left[\left(r_{1} \cdot f_{1}\right) / k_{\mathrm{p} 11}\right]$} \\
\hline 0.05 & $1.77 \cdot 10^{-4}$ & $8.72 \cdot 10^{-6}$ & $4.92 \cdot 10^{-2}$ \\
\hline 0.10 & $3.54 \cdot 10^{-4}$ & $8.26 \cdot 10^{-6}$ & $2.33 \cdot 10^{-2}$ \\
\hline 0.20 & $7.08 \cdot 10^{-4}$ & $7.34 \cdot 10^{-6}$ & $1.04 \cdot 10^{-2}$ \\
\hline 0.30 & $1.06 \cdot 10^{-3}$ & $6.42 \cdot 10^{-6}$ & $6.05 \cdot 10^{-3}$ \\
\hline 0.40 & $1.42 \cdot 10^{-3}$ & $5.51 \cdot 10^{-6}$ & $3.89 \cdot 10^{-3}$ \\
\hline 0.50 & $1.77 \cdot 10^{-3}$ & $4.59 \cdot 10^{-6}$ & $2.59 \cdot 10^{-3}$ \\
\hline 0.60 & $2.13 \cdot 10^{-3}$ & $3.67 \cdot 10^{-6}$ & $1.73 \cdot 10^{-3}$ \\
\hline 0.70 & $2.48 \cdot 10^{-3}$ & $2.75 \cdot 10^{-6}$ & $1.11 \cdot 10^{-3}$ \\
\hline 0.80 & $2.83 \cdot 10^{-3}$ & $1.84 \cdot 10^{-6}$ & $6.48 \cdot 10^{-4}$ \\
\hline 0.90 & $3.19 \cdot 10^{-3}$ & $9.18 \cdot 10^{-7}$ & $2.88 \cdot 10^{-4}$ \\
\hline 0.95 & $3.36 \cdot 10^{-3}$ & $4.59 \cdot 10^{-7}$ & $1.36 \cdot 10^{-4}$ \\
\hline
\end{tabular}

Tab. 5.10 Comparison between $\frac{r_{1} \cdot f_{1}}{k_{\mathrm{p} 11}}$ and $\frac{r_{2} \cdot f_{2}}{k_{\mathrm{p} 22}}$ in Eq. 5.2 for the system DA/MMA measured at $23{ }^{\circ} \mathrm{C} / 1000$ bar $\left(r_{1}=2.55\right.$ and $\left.r_{2}=0.29\right)$.

As was pointed out in section 5.3.1.1, in an acrylate/methacrylate copolymerization the overall propagation rate coefficient is close to the methacrylate value over a wide range of initial monomer feed ratios. This observation is valid for the systems MA/MMA, DA/DMA, MA/DMA, DA/MMA, and BA/MMA [29]. The value of $k_{\mathrm{p}, \text { copo }}$ remains almost constant at methacrylate concentrations above $f_{\text {mac }}=0.2$ and steeply increases at methacrylate mole fractions below $f_{\text {mac }}=0.1$ (toward the acrylate homopropagation value, $k_{\mathrm{p}, \mathrm{ac}}$, which exceeds $k_{\mathrm{p}, \mathrm{mac}}$ by more than one order of magnitude). The systems DA/DMA measured at $40{ }^{\circ} \mathrm{C} / 1000$ bar and DA/MMA at $23^{\circ} \mathrm{C} / 1000$ bar show a different type of composition dependence of $k_{\mathrm{p}, \text { copo. }}$ Figs. 5.11 and 5.17 indicate that $k_{\mathrm{p}, \text { copo }}$ increases within the entire range of $f_{\mathrm{mac}}$, although the increase is, as with the systems, particularly pronounced in the region of methacrylate mole fractions below $f_{\text {mac }}=0.2$.

The obtained kinetic data were analyzed in terms of the existing copolymerization $k_{\mathrm{p}}$ models. A brief overview of these models has been given in section 5.1.1.

For many years it was thought that the terminal model could describe the majority of copolymerization systems and it was thus considered to be a suitable basis for describing copolymerization kinetics. Subsequent studies have demonstrated that the terminal model adequately describes the instantaneous copolymer composition [21], and that it fails to do so only in a small number of cases, such as the styrene/acrylonitrile system [48]. 
To date, two alternative accounts of the origin of the IPUE model have been suggested known as the enthalpic and entropic models. In the enthalpic model, suggested by Fukuda et al. [18], it is proposed that the penultimate unit affects only the stability of the reacting radical but has no interaction with the reacting monomer [54]. Along these lines, the penultimate unit would (via an Evans-Polanyi rule) affect the activation energy, but the effect would be independent of the reacting monomer. In the entropic model, suggested by Heuts $e t$ al. [49], it is proposed that the penultimate unit affects the frequency factor of the propagation reaction. Provided the two monomers are not too dissimilar in size, the effect cancels for the monomer reactivity ratios and affects only the radical reactivity ratios. To date, discrimination between these two alternative mechanisms has not been possible because they predict the same type of kinetic behavior and differ only in their predicted values of the radical reactivity ratios.

In each of the copolymerization models the fitted model parameters have a physical meaning. Given this physical meaning, the expected temperature dependence of the parameters for each of these models may be summarized as follows: For the terminal model, the monomer reactivity ratios ( $r_{\mathrm{mac}}$ and $r_{\mathrm{ac}}$ ) would be expected to be slightly temperature dependent (based on chemical grounds). For the IPUE model, the radical reactivity ratios would be expected to be either slightly temperature sensitive (enthalpic model) or insensitive (entropic model) to temperature, depending upon whether or not they have an enthalpic component.

Fig. 5.3 has already demonstrated that the composition of the acrylate/methacrylate systems under investigation is adequately represented by the terminal model expression. The values of reactivity ratios obtained by applying Eq. 5.1 to the composition data are given in the first line of Tab. 5.11. It can be seen, that the $r_{\text {mac }}$ values of the various acrylate/methacrylate systems are close to each other, as are the $r_{\mathrm{ac}}$ data. The numbers for $r_{\mathrm{mac}}$ from fitting the composition data are in the range of $2.8 \pm 0.6$ and for $r_{\mathrm{ac}}$ are in the range of $0.31 \pm 0.12$. Throughout the entire composition range, the fraction of methacrylate polymerized, $F_{\text {mac }}$, exceeds the methacrylate monomer mole fraction, $f_{\text {mac }}$. This preference is most likely due to the higher stability of the radical formed upon the addition of a methacrylate monomer. The full line in Fig. 5.3 represents the fit of the entire data set to Eq. 5.1. The methacrylate and acrylate reactivity ratios referring to the fitted line in Fig. 5.1 are $r_{\mathrm{mac}}=2.55$ and $r_{\mathrm{ac}}=0.29$.

It should be noted, that for all systems under investigation, the $k_{\mathrm{p}, \text { copo }}$ values are also well represented by the terminal model if only the $k_{\mathrm{p} \text {,copo }}$ data is fitted. The reactivity ratios were obtained by fitting the experimental $k_{\mathrm{p} \text {,copo }}$ data to Eq. 5.2 and summarized in the second line of Tab. 5.11. Inspection of Tab. 5.11 shows the surprisingly low values of $r_{\text {mac }}$ in fitting the 
DA/MMA system and also the reduced (as compared to $r_{\text {mac }}$ from composition analysis) value of $r_{\text {mac }}$ for the systems DA/DMA and MA/DMA (both systems are measured at $40{ }^{\circ} \mathrm{C}$ and $1000 \mathrm{bar})$. In these systems the impact on $r_{\mathrm{mac}}$ is larger than that on $r_{\mathrm{ac}}$ as the $k_{\mathrm{p}, \text { copo }}$ effect is seen in the methacrylate-rich composition range and as $k_{\mathrm{p}, \text { mac }}$ is significantly below $k_{\mathrm{p}, \mathrm{ac}}$, which further enhances the importance of the methacrylate term in the denominator of Eq. 5.2.

In order to test the terminal model, the parameters obtained from fitting the terminal model to the $k_{\mathrm{p}, \text { copo }}$ data may be used to predict independently measured experimental data, such as the composition data. Fig. 5.18 shows the composition data of different acrylate/methacrylate systems. Lines in Fig. 5.18 represent fit of Eq. 5.1 with reactivity ratios obtained from fitting the $k_{\mathrm{p}, \text { copo }}$ data (second line in Tab. 5.11). It is seen that with reactivity ratios, taken from fitting only the $k_{\mathrm{p}, \text { copo }}$ data, systems MA/MMA measured at $23{ }^{\circ} \mathrm{C} / 1000$ bar and MA/DMA measured at $22{ }^{\circ} \mathrm{C} / 1000$ bar can be well described. In case of DA/DMA measured at $23{ }^{\circ} \mathrm{C} / 1000$ bar and MA/DMA measured at $40{ }^{\circ} \mathrm{C} / 1000$ bar reactivity ratios from the second line in Tab. 5.11 satisfactory represent experimental obtained composition data. For DA/MMA it was not possible to describe composition and $k_{\mathrm{p}, \text { copo }}$ data with similar reactivity ratios. 


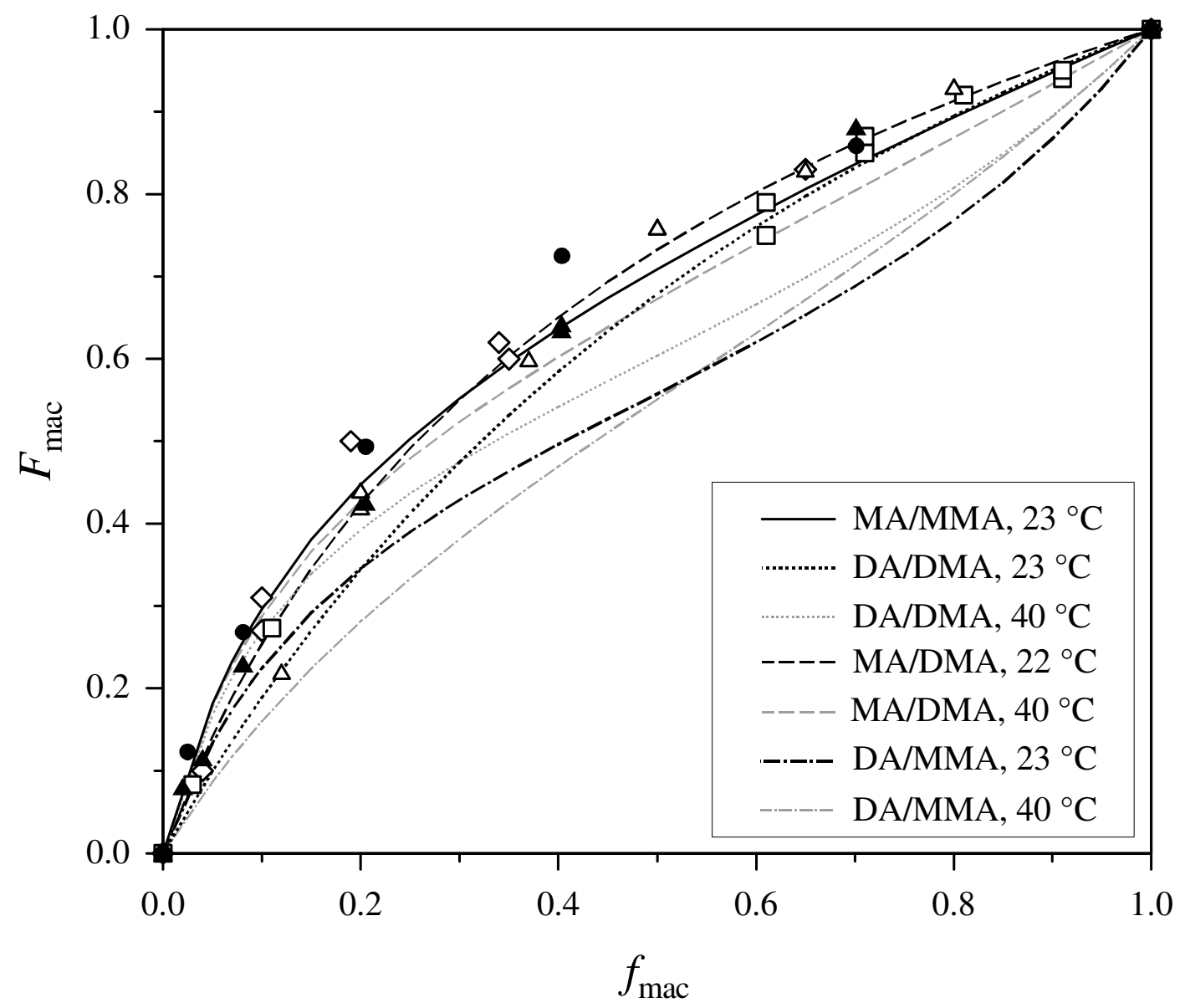

Fig. 5.18 Lewis-Mayo plot for all copolymerization systems under investigation: DA/DMA $\left(40{ }^{\circ} \mathrm{C}\right.$, squares), MA/DMA $\left(40{ }^{\circ} \mathrm{C}\right.$, diamonds), DA/MMA $\left(40{ }^{\circ} \mathrm{C}\right.$, triangles), DA/MMA $\left(23{ }^{\circ} \mathrm{C}\right.$, full triangles $)$, and MA/MMA $\left(23{ }^{\circ} \mathrm{C}\right.$, full circles). The lines represent the fit of Eq. 5.1 to different systems with reactivity ratios obtained by taking only the $k_{\mathrm{p}, \text { copo }}$ data (second line in Tab. 5.11). The composition data refers to 1000 bar.

The dotted lines in Figs. 5.7 and 5.14 represent $k_{\mathrm{p} \text {,copo }}$ values estimated via Eq. 5.2 using the reactivity ratios $r_{\mathrm{mac}}=2.55$ and $r_{\mathrm{ac}}=0.29$ taken from a Lewis-Mayo fit to the overall composition data. These graphs demonstrate that the terminal model fits rate and composition data in the MA/DMA at $22{ }^{\circ} \mathrm{C} / 1000$ bar and MA/MMA at $23{ }^{\circ} \mathrm{C} / 1000$ bar copolymerizations with almost the same reactivity ratios. There is a small discrepancy shown in Figs. 5.10 and 5.15 between calculated and experimental data within the systems DA/DMA measured at $23{ }^{\circ} \mathrm{C} / 1000$ bar and MA/DMA measured at $40{ }^{\circ} \mathrm{C} / 1000$ bar. It was found that the terminal model cannot adequately represent both sets of data in DA/DMA copolymerization at $40{ }^{\circ} \mathrm{C} / 1000$ bar and DA/MMA at $23^{\circ} \mathrm{C} / 1000$ bar simultaneously, as illustrated by the dotted 
lines in Figs. 5.11 and 5.17. In the latter case, the terminal model can adequately fit either the rate or the composition but not both data with the same reactivity ratios.

With the failure of the terminal model to fit both the composition and $k_{\mathrm{p} \text {,copo }}$ data for the systems DA/DMA measured at $40{ }^{\circ} \mathrm{C} / 1000$ bar and DA/MMA measured at $23{ }^{\circ} \mathrm{C} / 1000$ bar, the IPUE model was taken for a copolymerization modeling.

Reactivity ratios obtained by simultaneous fitting of the IPUE model to $k_{\mathrm{p} \text {,copo and }}$ composition data are given in the third line of Tab. 5.11. In the sixth line of Tab. 5.11, $r_{\mathrm{mac}}$ and $r_{\mathrm{ac}}$ were fixed to the values estimated from the terminal model fit to copolymer composition data and $k_{\mathrm{p}, \text { copo }}$ data was used to estimate $s_{\mathrm{mac}}$ and $s_{\mathrm{ac}}$.

Assuming that $s_{\mathrm{mac}} \cdot s_{\mathrm{ac}}$ in Eq. 5.5 is close to unity, the correlation $s_{\mathrm{ac}}=1 / s_{\mathrm{mac}}$ can be used for data analysis, which yields the results presented in the fourth line and in the seventh line (with monomer reactivity ratios taken from composition data) of Tab. 5.11. These values are close to the ones given in the third line of Tab. 5.11.

It was shown above that Eq. 5.9 instead of Eq. 5.2 could be used for the system DA/MMA. In this case, information about $s_{\mathrm{ac}}$ will be lost. For this reason correlation $s_{\mathrm{ac}}=1 / s_{\mathrm{mac}}$ was used. In the fifth line of Tab. 5.11 the results obtained from simultaneous fitting Eqs. 5.1 and 5.9 to the composition and $k_{\mathrm{p} \text {,copo }}$ data are presented. This procedure significantly affects values of radical reactivity ratios. 


\begin{tabular}{|c|c|c|c|c|c|c|c|c|c|}
\hline & & & MA/MMA & DA & & & MA & & MMA \\
\hline & & & $23^{\circ} \mathrm{C}$ & $23{ }^{\circ} \mathrm{C}$ & $40^{\circ} \mathrm{C}$ & $22{ }^{\circ} \mathrm{C}$ & $40^{\circ} \mathrm{C}$ & $23{ }^{\circ} \mathrm{C}$ & $40^{\circ} \mathrm{C}$ \\
\hline 1 & $F_{\mathrm{mac}}-f_{\mathrm{mac}}$ terminal & $r_{\mathrm{mac}}$ & 3.03 & - & 2.21 & - & 2.66 & 2.42 & 3.26 [29] \\
\hline 1 & model fit (Eq. 5.1) & $r_{\mathrm{ac}}$ & 0.20 & - & 0.38 & - & 0.25 & 0.29 & $0.43[29]$ \\
\hline 2 & $k_{\mathrm{p}, \mathrm{copo}}$ terminal & $r_{\mathrm{mac}}$ & 1.95 & 2.13 & 0.86 & 2.59 & 1.49 & 0.64 & 0.88 [29] \\
\hline 2 & model fit (Eq. 5.2) & $r_{\mathrm{ac}}$ & 0.21 & 0.48 & 0.22 & 0.31 & 0.21 & 0.30 & 0.53 [29] \\
\hline & & $r_{\mathrm{mac}}$ & 2.40 & 2.05 & 1.83 & 2.40 & 2.37 & 1.57 & 2.11 [29] \\
\hline 2 & IDUE fit & $r_{\mathrm{ac}}$ & 0.18 & 0.34 & 0.15 & 0.23 & 0.22 & 0.15 & 0.31 [29] \\
\hline 3 & IPUE III & $s_{\mathrm{mac}}$ & 1.46 & 1.25 & 3.15 & 1.17 & 1.77 & ind. & ind. [29] \\
\hline & & $s_{\mathrm{ac}}$ & 0.38 & 1.32 & 1.19 & 0.37 & 0.24 & 0.05 & 0.05 [29] \\
\hline & & $r_{\mathrm{mac}}$ & 2.39 & 2.05 & 1.88 & 2.39 & 2.34 & 1.52 & 1.99 \\
\hline 4 & IPUE fit & $r_{\mathrm{ac}}$ & 0.18 & 0.34 & 0.16 & 0.23 & 0.22 & 0.16 & 0.27 \\
\hline 4 & IPUE III & $s_{\mathrm{mac}}$ & 1.43 & 1.26 & 3.60 & 1.13 & 1.60 & 16.49 & 9.30 \\
\hline & & $s_{\mathrm{ac}}=1 / s_{\mathrm{mac}}$ & 0.70 & 0.79 & 0.28 & 0.88 & 0.62 & 0.06 & 0.11 \\
\hline & & $r_{\text {mac }}$ & 2.30 & 1.98 & 1.83 & 2.09 & 2.15 & 1.32 & 2.11 \\
\hline 5 & IPUE fit & $r_{\mathrm{ac}}$ & 0.15 & 0.23 & 0.13 & 0.16 & 0.18 & 0.11 & 0.23 \\
\hline 5 & (Eqs. 5.1 and 5.9) & $s_{\mathrm{mac}}$ & 1.38 & 1.35 & 2.82 & 1.02 & 1.37 & 3.42 & 5.92 \\
\hline & & $s_{\mathrm{ac}}=1 / s_{\mathrm{mac}}$ & 0.72 & 0.74 & 0.35 & 0.98 & 0.73 & 0.29 & 0.17 \\
\hline & $\begin{array}{c}\text { IPUE fit } \\
r_{\mathrm{mac}} \text { and } r_{\mathrm{ac}} \text { are }\end{array}$ & $r_{\text {mac }}$ & 2.55 & 2.55 & 2.55 & 2.55 & 2.55 & 2.55 & 2.55 \\
\hline 6 & $\begin{array}{l}r_{\mathrm{mac}} \text { and } r_{\mathrm{ac}} \text { are } \\
\text { from the overall }\end{array}$ & $r_{\mathrm{ac}}$ & 0.29 & 0.29 & 0.29 & 0.29 & 0.29 & 0.29 & 0.29 \\
\hline 6 & from the overall & $s_{\text {mac }}$ & 1.03 & 1.85 & 2.56 & 1.02 & 1.63 & ind. & ind. \\
\hline & $\begin{array}{c}F_{\text {mac }}-f_{\text {mac }} \text { terminal } \\
\text { model fit }\end{array}$ & $s_{\mathrm{ac}}$ & 1.12 & 0.54 & ind. & 0.99 & 0.16 & 0.05 & 0.14 \\
\hline & $\begin{array}{c}\text { IPUE fit, } \\
r_{\mathrm{mac}} \text { and } r_{\mathrm{ac}} \text { are }\end{array}$ & $r_{\mathrm{mac}}$ & 2.55 & 2.55 & 2.55 & 2.55 & 2.55 & 2.55 & 2.55 \\
\hline 7 & $\begin{array}{l}r_{\text {mac and }} r_{\mathrm{ac}} \text { are } \\
\text { from the overall }\end{array}$ & $r_{\mathrm{ac}}$ & 0.29 & 0.29 & 0.29 & 0.29 & 0.29 & 0.29 & 0.29 \\
\hline & $\begin{array}{l}\text { from the overall } \\
F-f_{m x} \text { terminal }\end{array}$ & $s_{\mathrm{mac}}$ & 1.04 & 1.84 & 3.01 & 1.02 & 1.39 & 4.60 & 8.62 \\
\hline & $\begin{array}{c}F_{\text {mac }}-f_{\text {mac }} \text { terminal } \\
\text { model fit }\end{array}$ & $s_{\mathrm{ac}}=1 / s_{\mathrm{mac}}$ & 0.96 & 0.55 & 0.33 & 0.98 & 0.72 & 0.22 & 0.12 \\
\hline
\end{tabular}

Tab.5.11 Reactivity ratios obtained by fitting the experimental composition and propagation rate data of acrylate/methacrylate copolymerizations to different models. The data refers to 1000 bar ("ind." says that the value is indeterminate).

In the system MA/DMA measured at $22{ }^{\circ} \mathrm{C} / 1000$ bar and $40{ }^{\circ} \mathrm{C} / 1000$ bar $s_{\text {mac }}$ is higher than $s_{\mathrm{ac}}$, which is in good agreement to what has been already reported $[29,30]$. In the system DA/DMA investigated at $40{ }^{\circ} \mathrm{C} / 1000$ bar $s_{\text {ac }}$ seems to be little sensitive to the IPUE fitting procedure. It should be noted, that with radical reactivity ratios equal to unity the IPUE model 
expression for the average propagation rate coefficient (Eq. 5.4) collapses to the terminal model expression. Heuts et al. [49] pointed out that steric effects are generally of equal, if not of large importance as electronic effects. The size of the substituent determines the internal barriers (i.e., degree of hindrance) to internal motions in the transition state of the reaction. The greater the hindrance is, the smaller is the Arrhenius frequency factor. The effect of the hindrance of the penultimate unit may cancel if the two comonomers are not too dissimilar in size and shape. It follows that if two monomers are very similar in size, the propagation rate coefficient can be predicted by the terminal model. Therefore it comes as no surprise that the terminal model works rather well in the systems MA/MMA and DA/DMA investigated at $23^{\circ} \mathrm{C}$ and 1000 bar.

Only for the system DA/MMA at both temperatures (Tab. 5.11) the IPUE model does not provide reasonable numbers for the radical reactivity ratios with meaningful accuracy.

It was noted by Coote et al. [24] that a central difficulty in obtaining accurate values for radical reactivity ratios is the fact that these parameters are extremely sensitive to small variations in the data.

It was already mentioned above that the intramolecular chain transfer reaction (backbiting) plays an important role in homopolymerization of acrylates [2]. Inspection of the literature shows that the reactivity ratios obtained from composition data of copolymerization of butyl acrylate with styrene $\left(r_{\mathrm{St}}=0.81\right.$ and $\left.r_{\mathrm{BA}}=0.20\right)$ [50] are close to the reactivity ratios obtained in copolymerization of butyl acrylate dimer (species that forms radicals of similar structure to the mid-chain radicals) with styrene $\left(r_{\mathrm{St}}=0.73\right.$ and $\left.r_{\mathrm{BAD}}=0.11\right)$ [51]. This gives a strong support that the mid-chain radicals have a small influence on the composition data but they should have an impact on the overall propagation rate.

In section 7 it will be presented, that the amount of mid-chain radicals in DA homopolymerization is higher than in BA homopolymerization under PLP conditions. In section 9 it will be shown that the intramolecular chain transfer coefficient, $k_{\mathrm{bb}}$, for DA is higher than for BA. It was shown that $k_{\mathrm{bb}}$ for BA is higher than for MA [45,52]. One can expect that in DA-containing copolymer systems thus should be more mid-chain radicals at high mole fractions of acrylate than in MA-containing systems. This may cause difficulties in application of different copolymerization models to the experimental data obtained in DA copolymerization. Resulting from backbiting, tertiary mid-chain radicals will propagate at much slower rate than the secondary radicals of acrylates. In acrylate/methacrylate copolymerization systems two types of tertiary radicals may be present: propagating radicals of methacrylates and mid-chain radicals originating from backbiting. 
In the DA/MMA system the monomers have very different sizes of the ester group (very bulky dodecyl chain in case of DA and rather small methyl group in case of MMA). It may be assumed that in the DA/MMA system measured even at $23{ }^{\circ} \mathrm{C} / 1000$ bar, tertiary radicals of both types (propagating radicals of methacrylates and mid-chain radicals) have a similar rate of propagation. If a mid-chain radical has different monomer units (DA and/or MMA) to its right and left sides this may cause a failure of the copolymerization models proposed so far. On the other hand, in case of DA/DMA both monomers have the same bulky ester group that can wrap around a chain.

ESR studies discussed in sections 7 and 9 show high activation energy for backbiting $\left(E_{\mathrm{A}}=52 \mathrm{~kJ} \cdot \mathrm{mol}^{-1}\right)$. Thus, with increasing temperature, the amount of mid-chain radicals will significantly increase making the situation more complicated. In order to test whether the mid-chain radicals have a large influence on $k_{\mathrm{p} \text {,copo }}$ in the copolymerization system DA/MMA the IPUE model was fitted to the composition and $k_{\mathrm{p} \text {,copo }}$ data in the range of $0.6<f_{\mathrm{mac}}<1$. Unfortunately, no improvement in reactivity ratios was determined.

In Tab. 5.12 is given example for BA/MMA.

For the system BA/MMA measured at $50{ }^{\circ} \mathrm{C}$ and ambient pressure, reported by Hutchinson $e t$ al. [30], the terminal model can be used for describing the composition data. The IPUE model was capable of describing the $k_{\mathrm{p}, \text { copo }}$ and composition data simultaneously. Buback et al. [29] reported that the IPUE model can be satisfactory fitted to the rate and composition data simultaneously for the system BA/MMA measured at $40{ }^{\circ} \mathrm{C} / 1000 \mathrm{bar}$. 


\begin{tabular}{|c|c|c|c|c|}
\hline & & & \multicolumn{2}{|c|}{ BA/MMA } \\
\hline & & & $40^{\circ} \mathrm{C} / 1000$ bar & $50{ }^{\circ} \mathrm{C} / 1$ bar \\
\hline \multirow{2}{*}{1} & $F_{\text {mac }}-f_{\text {mac }}$ terminal & $r_{\mathrm{mac}}$ & $2.24^{\mathrm{a}}[30]$ & $2.24[30]$ \\
\hline & model fit (Eq. 5.1) & $r_{\mathrm{ac}}$ & $0.41^{\mathrm{a}}[30]$ & $0.41[30]$ \\
\hline \multirow{2}{*}{2} & $k_{\mathrm{p}, \mathrm{copo}}$ terminal & $r_{\mathrm{mac}}$ & $1.52[29]$ & - \\
\hline & model fit (Eq. 5.2) & $r_{\mathrm{ac}}$ & $0.44[29]$ & - \\
\hline \multirow{4}{*}{3} & \multirow{4}{*}{ IPUE fit } & $r_{\mathrm{mac}}$ & $2.47[29]$ & $2.16[30]$ \\
\hline & & $r_{\mathrm{ac}}$ & 0.38 [29] & $0.40[30]$ \\
\hline & & $s_{\mathrm{mac}}$ & $2.06[29]$ & $10.4[30]$ \\
\hline & & $s_{\mathrm{ac}}$ & ind. [29] & $0.03[30]$ \\
\hline
\end{tabular}

${ }^{\mathrm{a}}$ From data for ambient pressure and $50{ }^{\circ} \mathrm{C}[30]$

Tab. 5.12 Reactivity ratios obtained by fitting the experimental composition and propagation rate data of BA/MMA to different models ("ind." says that the value is indeterminate).

It goes without saying that while the IPUE model can successfully describe the available experimental data, other models (such as, for example, the bootstrap model introduced by Harwood [53]) can also be fitted to the same data. A large number of these alternative models can be successfully fitted to the same experimental values and hence attempts to discriminate between the alternative models on this basis have been largely unsuccessful [54].

To summarize, as far as it is desirable to choose a model with the smallest possible number of adjustable parameters, but proving an adequate fit to the data [55], the terminal model was separately fitted to the $k_{\mathrm{p} \text {,copo }}$ and composition data for all systems tested in this study. It was shown that the terminal model fits the data with reasonable accuracy in the systems MA/MMA measured at $23{ }^{\circ} \mathrm{C} / 1000$ bar, MA/DMA measured at $22{ }^{\circ} \mathrm{C} / 1000$ bar, and DA/DMA measured at $23{ }^{\circ} \mathrm{C} / 1000$ bar but fails in the systems DA/DMA measured at $40{ }^{\circ} \mathrm{C} / 1000$ bar, MA/DMA measured at $40{ }^{\circ} \mathrm{C} / 1000$ bar, and DA/MMA measured at both $23{ }^{\circ} \mathrm{C} / 1000$ bar and $40{ }^{\circ} \mathrm{C} / 1000$ bar.

The explanation for the failure of the terminal and IPUE models proposed in this work was based on the presence of mid-chain radicals originating from the intramolecular chain transfer reaction playing an important role in homopolymerization of acrylates even at moderately high temperatures. Another explanation was proposed by Buback et al. [29]. They assumed that the "pen"-penultimate unit of a propagating radical might have an influence on copolymerization kinetics of DA-containing systems. To date, it is not possible to give 
preference to one of these explanations unambiguously. It may well be that both effects play a role in acrylate/methacrylate copolymerizations. 


\subsection{REFERENCES}

[1] Herk, A. M. v. Macromol. Rapid Commun. 2001, 22, 687.

[2] Asua, J. M.; Beuermann, S.; Buback, M.; Castignolles, P.; Charleux, B.; Gilbert, R. G.; Hutchinson, R. A.; Leiza, J. R.; Nikitin, A. N.; Vairon, J. P.; Herk, A. M. v. Macromol. Chem. Phys. 2004, 205, 2151.

[3] Busch, M.; Wahl, A. Macromol. Theory Simul. 1998, 7, 217.

[4] Ahmad, N. M.; Heatley, F.; Lovell, P. A. Macromolecules 1998, 31 (9), 2822.

[5] Heatley, F.; Lovell, P. A.; Yamashita, T. Macromolecules 2001, 34 (22), 7636.

[6] Britton, D.; Heatley, F.; Lovell, P. A. Macromolecules 1998, 31 (9), 2828.

[7] Kajiwara, A.; Kamachi, M. ACS Symposium Series 2003, 854 (Advances in Controlled/Living Radical Polymerization), 86.

[8] Arzamendi, G.; Plessis, C.; Leiza, J. R.; Asua, J. M. Macromol. Theory Simul. 2003, 12,315 .

[9] Alfrey, T.; Goldfinger, G. J. Chem. Phys. 1944, 12, 205.

[10] Mayo, F. R.; Lewis, F. M. J. Am. Chem. Soc. 1944, 66, 1594.

[11] Maxwell, I. A.; Aerdts, A. M.; German, A. L. Macromolecules 1993, 26, 1956.

[12] Fukuda, T.; Ma, Y.-D.; Inagaki, H. Macromolecules 1985, 18, 17.

[13] Hill, D. J. T.; O'Donnell, H.; O'Sullivan, P. W. Macromolecules 1982, 15, 960.

[14] Fukuda, T.; Ma, Y.-D., Inagaki, H. Macromolecules 1985, 18, 26.

[15] Ma, Y.-D.; Kubo, P.-S.; Fukuda, T. Macromolecules 1993, 26, 6766.

[16] Davis, T. P.; O'Driscoll, K. F.; Piton, M. C.; Winnik, M. A. Polym. Int. 1991, 24, 65.

[17] Fukuda, T.; Ma, Y.-D.; Kubo, M.; Inagaki, H. Macromolecules 1991, 24, 370.

[18] Fukuda, T.; Ma, Y.-D.; Inagaki, H. Macromol. Chem., Rapid. Commun. 1987, 8, 495.

[19] Fukuda, T.; Ma, Y.-D.; Kubao, K; Takada, A. Polym. J. (Tokyo) 1989, 12, 1003.

[20] Merz, E.; Alfrey, T.; Goldfinger, G. J. Polym. Sci. 1946, 1, 75.

[21] Fukuda, T; Kubo, K.; Ma, Y.-D. Prog. Polym. Sci. 1992, 17, 875.

[22] Hutchinson, R. A.; McMinn, J. H.; Paquet, D. A. Jr.; Beuermann, S.; Jackson, C. Ind. Eng. Chem. Res. 1997, 36, 1103.

[23] Coote, M. L.; Zammit, M. D.; Davis, T. P.; Willet, G. D. Macromolecules 1997, 30, 8190 .

[24] Coote, M. L.; Johnston, L. P. M.; Davis, T. P. Macromolecules 1997, 30, 8191.

[25] Buback, M.; Busch, M.; Lämmel, R. DECHEMA Monogr. 1995, 131, 569.

[26] Mahabadi, H. K. Macromolecules 1985, 18, 1319. 
[27] Olaj, O.F.; Vana, P. Macromol. Rapid. Commun. 1998, 19, 533.

[28] Arias, C; Lopez-Gonzalez, M. M. C.; Fernandez-Garcia, M.; Barrales-Rienda, J. M.; Madruga, E. L. Polymer. 1993, vol. 34, 8, 1786.

[29] Buback, M.; Feldermann, A.; Barner-Kowollik, C.; Lacik, I. Macromolecules. 2001, 34, 5439 .

[30] Hutchinson, R. A.; McMinn, J. H.; Paquet, D. A. Jr.; Beuermann, S.; Jackson, C. Ind. Eng. Chem. Res. 1997, 36, 1103.

[31] Varma, I. K.; Nair, M. V.; Karan, V. K.; Varma, D. S. Thermochimica Acta 1989, 142, 189.

[32] Advanced Organic Chemistry, March, J. $3^{\text {rd }}$ ed., J. Wiley and Sons: New York, 1985

[33] Kowollik, C. Ph. D. Thesis, Göttingen, 1999.

[34] Hutchinson, R. A.; Paquet, D. A. Jr.; McMinn, J. H.; Beuermann, S.; Fuller, R. E.; Jackson, C. DECHEMA Monogr. 1995, 131, 467.

[35] Madruga, E. L.; Fernandez-Garcia, M. Macromol. Chem. Phys. 1996, 197, 1185

[36] Schweer, J.; Saranecki, J. Macromolecules 1995, 28, 4080.

[37] Beuermann, S.; Paquet, D. A.; McMinn, J. H.; Hutchinson, R. A. Macromolecules 1996, 29, 4206.

[38] Arzamendi, G.; Plessis, C.; Leiza, J. R.; Asua, J. M. Macromol. Theory Simul. 2003, 12,315 .

[39] Plessis, C.; Arzamendi, G.; Alberdi, J. M.; van Herk, A. M.; Leiza, J. R.; Asua, J. M. Macromol. Rapid. Commun. 2003, 24, 173.

[40] Plessis, C.; Arzamendi, G.; Leiza, J. R.; Schoonbrood, H. A. S.; Charmont, D.; Asua, J. M. Macromolecules 2000, 33, 4.

[41] Lyons, R.A.; Hutovic, J.; Piton, M. C.; Christie, D. I.; Clay, P. A.; Manders, B. G.; Kable, S. H.; Gilbert, R. G. Macromolecules 1996, 29, 1918.

[42] Ahmad, N. M.; Heatley, F.; Lovell, P. A. Macromolecules 1998, 31, 2822.

[43] Chiefari, J.; Jeffery, J.; Mayadunne, R. T. A.; Moad, G.; Rizzardo, E.; Thang, S. H. Macromolecules 1999, 32, 7700.

[44] Tanaka, K.; Yamada, B.; Fellows, C. M.; Gilbert, R. G.; Davis, T. P.; Yee, L. H.; Smith, G. B.; Rees, M. T. L.; Russell, G. T. J. Polym. Sci. Part A, Polym. Chem. 2001, $39,3902$.

[45] Müller, M. Ph. D. Thesis, Göttingen, 2005. 
[46] Nikitin, A. N.; Castignolles, P.; Charleux, B.; Vairon, J.-P. Macromol. Rapid Commun. 2003, 24, 778.

[47] Beuermann, S.; Buback, M.; Davis, T. P.; Gilbert, R. G.; Hutchinson, R. A.; Kajiwara, A.; Klumperman, B.; Russell, G. T. Macromol. Chem. Phys. 2000, 201, 1355.

[48] Klumperman, B.; Kraeger, I. R. Macromolecules 1994, 27, 1529.

[49] Heuts, J. P. A.; Gilbert, R. G.; Maxwell, I. A. Macromolecules 1997, 30, 726.

[50] Wahl, A. Ph. D. Thesis, Göttingen, 2000.

[51] Trumbo, D. L.; Zander, R. A. J. Polym. Sci. Part A, Polym. Chem. 1991, 29, 1053.

[52] Stiesel, B. Diploma Thesis, Göttingen, 2004.

[53] Harwood, H. J.; Macromol. Chem., Macromol. Symp. 1987, 10/11, 331.

[54] Coote, M. L.; Davis, T. P. Prog. Polym. Sci. 1999, 24, 1217.

[55] Handbook of Radical Polymerization, Matyjaszewski, K.; Davis, T. P., Eds.; WileyInterscience: New York, 2002. 


\section{Termination}

\subsection{INTRODUCTION}

The aim of most kinetic experimental methods has always been to determine accurate values for the individual rate coefficients that govern the free-radical polymerization process. Classical methods to investigate free-radical polymerization kinetics have involved the determination of individual rate parameters for propagation $\left(k_{\mathrm{p}}\right)$ and termination $\left(k_{\mathrm{t}}\right)$ from ratios of these parameters. New ways had to be explored which would give access to both $k_{\mathrm{p}}$ and / or $k_{\mathrm{t}}$ from a single experiment.

A major improvement in the determination of kinetic parameters came with the advent of pulsed lasers into kinetic studied in the 80's [1]. The PLP-SEC technique enables the determination of individual $k_{\mathrm{p}}$ values, not coupled with $k_{\mathrm{t}}$. This method is not only virtually model free, it has also proven to yield highly accurate and precise $k_{\mathrm{p}}$ values for, e.g., styrene and different methacrylates $[2,3,4]$. Beside propagating rate coefficients, pulsed lasers have also been used in the determination of termination rate coefficients. The SP-PLP (single pulse-pulsed laser polymerization) technique has been introduced to measure termination rate coefficients for various homo- and copolymerizations $[5,6,7,8]$. The measurement of $k_{\mathrm{t}}$ by this method is indirect in that the time-dependence of monomer concentration, rather than that of radical concentration, is measured. If additional information on either $k_{\mathrm{p}}$ (from PLP-SEC) or the primary radical concentration is available, termination rate coefficient can be determined. In this work a new method, SP-PLP-ESR (single pulse-pulsed laser polymerization-electron spine resonance) is presented for the determination of termination rate coefficients. The new technique applies the principle of the conventional SP-PLP in conjunction with time-resolved measurement of radical concentration decay using electron spin resonance (ESR) spectroscopy.

It is the goal of this chapter to shine light upon the exact dependence of free-radical termination rate coefficients upon the chain length of the terminating radical species. As $k_{\mathrm{t}}$ values are highly system specific, the most simple and easily reproducible polymerization system is chosen: termination reaction at low conversions in bulk polymerization. In this section termination kinetics of dodecyl methacrylate [9], cyclohexyl methacrylate and benzyl methacrylate free-radical homopolymerizations as a function of conversion and temperature (for benzyl methacrylate) are discussed. The results are explained using a "composite model" proposed in literature [10] for chain-length dependence of $k_{\mathrm{t}}$. 


\subsection{TERMINATION IN HOMOPOLYMERIZATION}

The termination reaction in free-radical polymerization is a highly complex reaction [1]. Despite of the availability of accurate values of $k_{\mathrm{p}}$, the fundamental knowledge of many aspects of the termination reactions is still far from being complete. In the termination reaction $k_{\mathrm{t}}$ is influenced by a multitude of different factors. To understand this, it is useful to recall the diffusion-controlled nature of termination. All parameters that can exert an effect on the mobility of radicals will have an effect on the overall rate of termination and the chainlength of the radicals involved in the termination reaction is one of these parameters. As large coils are known to diffuse less rapidly than small ones, the chain length of radicals can be expected to have a profound effect on the termination kinetics. Therefore, one rate coefficient $k_{\mathrm{t}}$ cannot describe all termination interactions occurring in a polymerizing system at any instant, because radicals of a variety of sizes are present. Thus one must properly describe termination in terms of $k_{\mathrm{t}}(i, j)$, the rate coefficient for termination between radicals of degree of polymerization $i$ and $j$ respectively. In carrying out a free-radical experiment, one will invariably measure the overall rate of termination, which is the sum of the rates of termination for all possible pairs of radical seizes. Thus $\left\langle k_{\mathrm{t}}\right\rangle$, the overall or average rate coefficient for termination, defined such that $\left\langle k_{\mathrm{t}}>\right.$ replaces $k_{\mathrm{t}}$ in Eq. 3.5, is related to the more physically meaningful $k_{\mathrm{t}}(i, j)$ values by [11]

$$
<k_{\mathrm{t}}>=\sum_{i} \sum_{j} k_{\mathrm{t}}(i, j) \frac{c_{\mathrm{R}}(i) \cdot c_{\mathrm{R}}(j)}{\left(\sum_{i} c_{\mathrm{R}}(i)\right)^{2}}
$$

Here $c_{\mathrm{R}}(i)$ denotes the concentration of radicals of chain length $i$ and $\sum_{i} c_{\mathrm{R}}(i)$ is the total freeradical concentration. Note that Eq. 6.1 is not a steady-state equation: it applies at all instants, regardless of whether or not the system is in a steady state. Therefore Eq. 6.1 means that under non-steady state conditions where the radical chain-length distribution is changing considerably with time, $<k_{\mathrm{t}}>$ may vary with time. Thus the measured $k_{\mathrm{t}}$ can only be identified with some sort of time-averaged value. The exact value of this averaging over time is usually complicated and depends on the details of the experiment [12,13]. Single pulsed laser experiments, in which the radical chain-length distribution, to a good approximation, is monodisperse (unless chain transfer process comes into play), would appear to offer the best perspectives for overcoming this difficulty and enabling unambiguous measurement of $k_{\mathrm{t}}(i, i)$ $[14,15]$. 
A simple and frequently used way of taking the chain-length dependence of $k_{\mathrm{t}}$ into account is by employing the so-called power-law model, which assumes that $k_{\mathrm{t}}$ varies with radical chain length $i$ according to:

$$
k_{\mathrm{t}}(i, i)=k_{\mathrm{t}}^{0} \cdot i^{-\alpha}
$$

Here $k_{\mathrm{t}}(i, i)$ is the rate coefficient for termination between two polymerizing radicals of degree of polymerization $i . k_{\mathrm{t}}^{0}$ can be identified as the rate coefficient for very small free-radicals while $\alpha \geq 0$ is an exponential which quantifies the chain-length dependence of the termination rate coefficient: the higher its value, the stronger the decline of $k_{\mathrm{t}}(i, i)$ with increasing radical size.

If center-of-mass diffusion is the rate-determining step in the termination reaction, an exponent of $0.5-0.6$ results, depending on the quality of the solvent [37]. This result can be explained by considering the translational diffusion properties of the polymer coil. The diffusion coefficient of the polymer coil is inversely proportional to its hydrodynamic radius. If the hydrodynamic radius is equated with the radius of gyration, $\left\langle s^{2}\right\rangle^{0.5}$, it can be inferred that for a theta solution $\left\langle s^{2}\right\rangle \propto i$ and for an athermic solution $\left\langle s^{2}\right\rangle \propto i^{1.176}$ [16]. This leads directly to the chain-length dependence of the diffusion coefficient of $D \propto i^{-0.5}$ for athermic solutions. According to various theories for diffusion controlled reactions, the diffusion coefficient is directly proportional to the termination rate coefficient.

The segmental diffusion is generally chain-length independent. However, a slight chainlength dependence of the termination coefficient of close to $\alpha=0.16$ is predicted for athermic solvents, due to thermodynamic shielding effects of the chain. The chain shields its own reactive center because of its size from the second chain, which displays a similar shielding effect. Experimentally, evidence has been gathered for both exponents, 0.1-0.2 for segmental diffusion and $0.5-0.6$ for center-of-mass diffusion, depending on the size of the macroradicals undergoing termination [37]. 


\section{Composite Model}

It was suggested in literature [10] to use a "composite model" for dilute solutions (very low conversion), which distinguishes between short and long radicals. This model includes the ideas of both center-of-mass diffusion $[17,42]$ and chain-end encounter $[18,19,28]$. Thus Eq. 6.2 can be used only for "short" radicals with $i \leq i_{\mathrm{c}}$, where $i_{\mathrm{c}} \approx 100$ appeals as the size at which a radical might start displaying long-chain behavior with regard to termination. For "long" radicals the authors propose that

$$
k_{\mathrm{t}}(i, i)=k_{\mathrm{t}}^{0} \cdot\left(i_{\mathrm{c}}\right)^{-\alpha_{1}+\alpha_{2}} \cdot i^{-\alpha_{2}}
$$

where $\alpha_{1}$ belongs to "short" radicals and $\alpha_{2}$ to "long" radicals. For short chain length $\alpha$ is predicted to be 0.5 [20,29] (length ca. 100 or less) and falls off to $0.16[18,19,28]$ for long radicals. The lager $\alpha$ value represents the appreciable initial lowering of $k_{\mathrm{t}}$ from experimental radical-radical combination rate coefficients of two small radicals up to the onset of the weaker chain-length dependence that is expected for larger coiled radicals.

\subsection{MEASUREMENTS OF $\boldsymbol{k}_{\mathrm{t}}$ VIA SP-PLP-ESR}

All experiments have been carried out at ambient pressure. Because of the chain-length dependence of the termination rate coefficient, it is rather difficult to report tabulated values of $k_{\mathrm{t}}$. However, it is possible to give chain-length averaged $k_{\mathrm{t}}$ values for a specific monomer conversion, $\left\langle k_{\mathrm{t}}\right\rangle$. It is important to note that for many of the chain-length averaged $k_{\mathrm{t}}$ values reported in literature, it is unclear to which chain-length region they correspond. In the present study all averaged $k_{\mathrm{t}}$ values are given for the chain length region $1<i<1000$ and for monomer conversions below 30 per cent. These termination rate coefficients have been deduced by the SP-PLP-ESR technique. The propagation rate coefficients were determined by independent PLP-SEC experiments. All SP-PLP-ESR experiments have been carried out with MMMP as the photoinitiator at low (initial) radical concentrations, close to $10^{-6} \mathrm{~mol} \cdot \mathrm{L}^{-1}$. The termination rate coefficients in this section always refer to low conversions, if not explicitly stated otherwise. 


\subsubsection{DEPENDENCE OF $\boldsymbol{k}_{\mathrm{t}}$ (DMA) ON MONOMER CONVERSION}

Fig. 6.1 shows a typical 13-line ESR spectrum for low-conversion DMA polymerization.

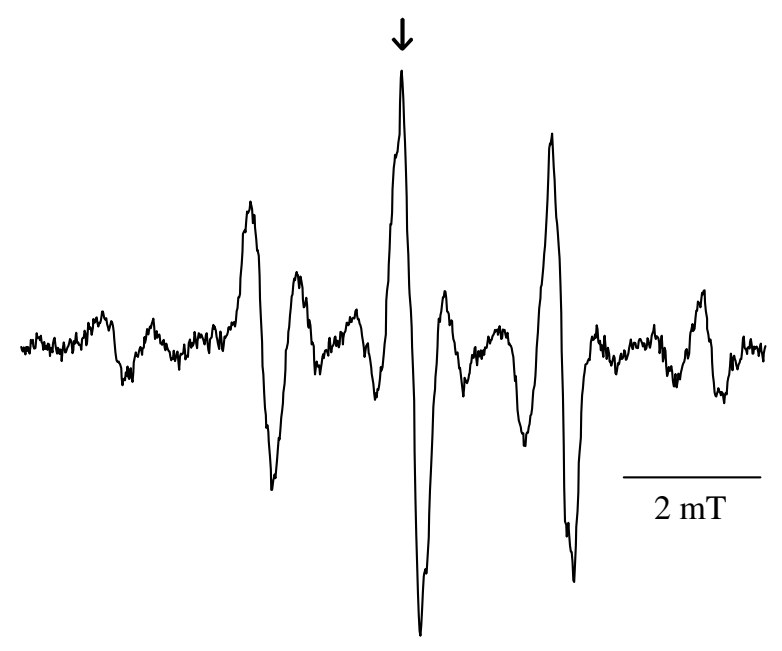

Fig. 6.1 Spectrum of DMA macroradical from quasistationary polymerization initiated by laser pulses applied at a repetition rate of $20 \mathrm{~Hz}$ and carried out at $0{ }^{\circ} \mathrm{C}$, $c_{\text {MMMP }}=25.5 \cdot 10^{-3} \mathrm{~mol} \cdot \mathrm{L}^{-1}$.

Fig. 6.2 shows ESR spectra for the polymerization of DMA at $0{ }^{\circ} \mathrm{C}$ and different degrees of monomer conversions. At these low conversions 13-line spectra $(5+8)$ were obtained. Kamachi and Kajiwara attributed the 13-line spectrum of methyl methacrylate to two conformations that can be converted into each other by rotation around the $\mathrm{C}_{\alpha}-\mathrm{C}_{\beta}$ bond at the free-radical end [21,22]. An increase in the degree of polymerization reveals the broadening of the moderate inner 8 lines in the 13-line spectrum. At high conversions, instead of 8 only 4 broad inner lines were obtained. The authors explain the origin of the 9-line spectra $(5+4)$ is to be due to restricted motions of macroradicals. 


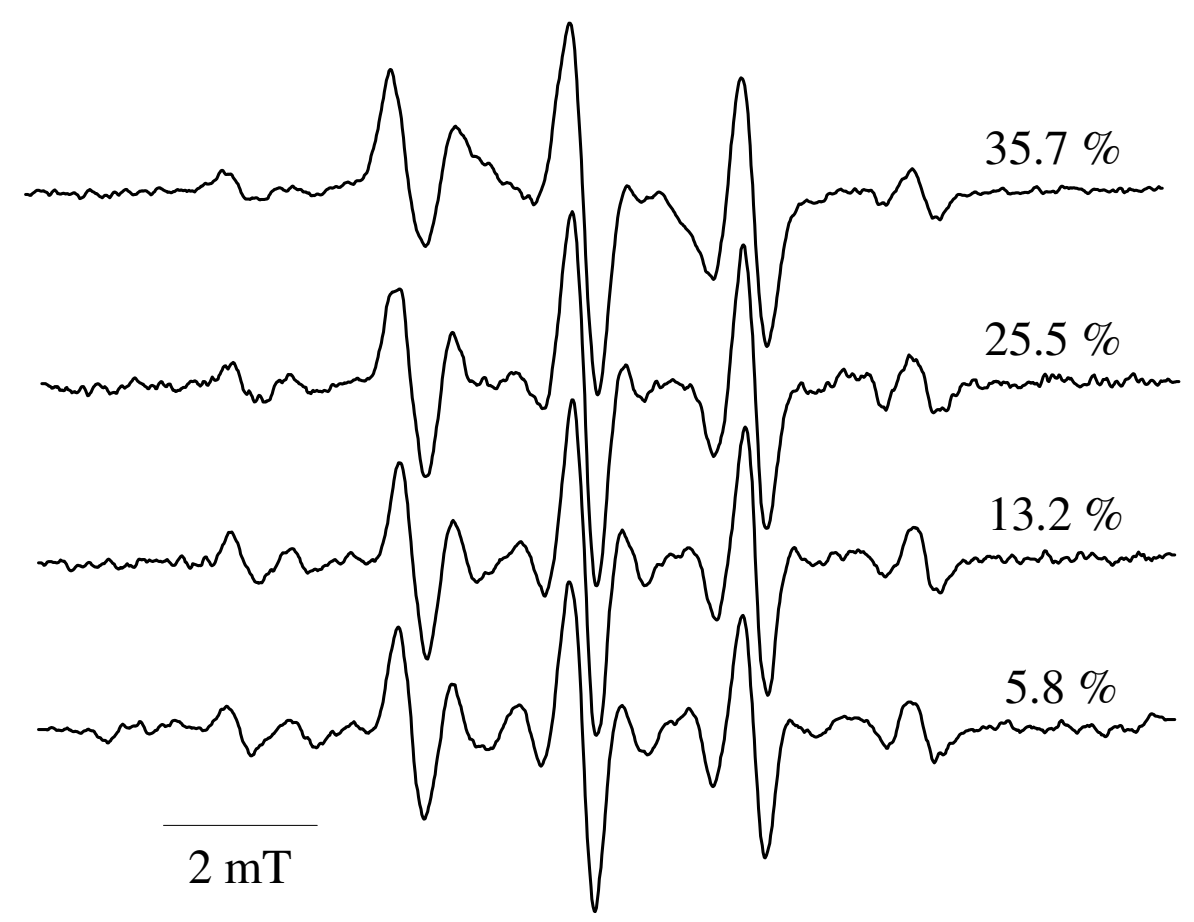

Fig. 6.2 Spectra of DMA macroradicals from quasistationary polymerization obtained under continuous UV irradiation at different conversions at $0{ }^{\circ} \mathrm{C}$, $c_{\text {MMMP }}=10.2 \cdot 10^{-3} \mathrm{~mol} \cdot \mathrm{L}^{-1}$.

Nine-line spectra, as reported for methacrylates by other groups [23], were observed for DMA at conversions above $30 \%$. In the present study the conversion range of SP-PLP-ESR experiments was below 30 per cent. In these experiments, the peak height of the line with maximum intensity (indicated by the arrow in Fig. 6.1), which is a measure of radical concentration (see Experimental Part), was recorded at constant magnetic field. The DMA conversion induced by a single laser pulse is typically around 0.05 per cent.

Shown in Fig. 6.3 is a $c_{\mathrm{R}}(t) / c_{\mathrm{R}}{ }^{0}$ trace, with $c_{\mathrm{R}}{ }^{0}$ being the radical concentration at time $t=0$, measured for DMA polymerization at $0{ }^{\circ} \mathrm{C}$. 


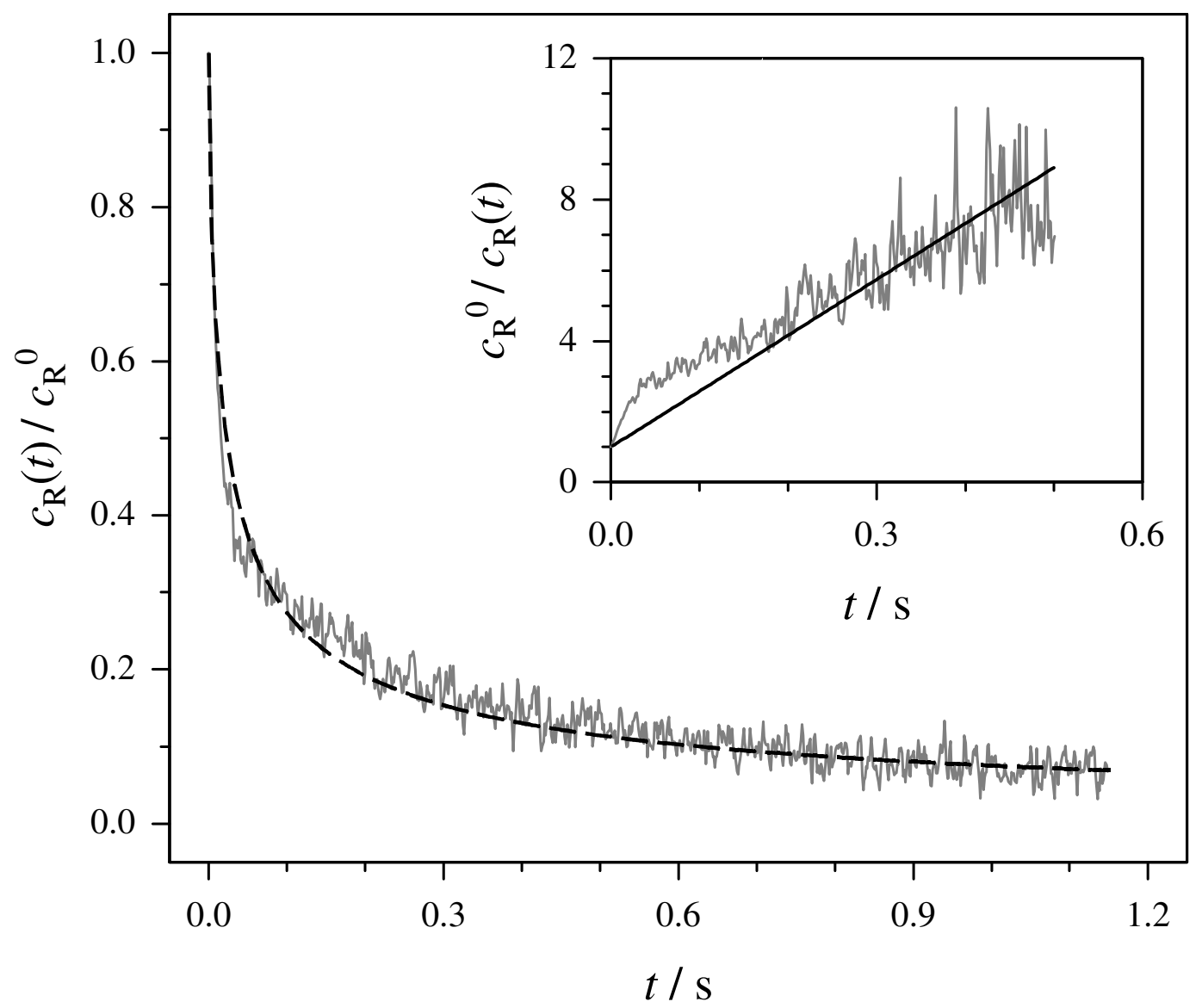

Fig. 6.3 Decay in radical concentration after applying a laser pulse at $t=0$ as measured by ESR for a DMA bulk polymerization at $0{ }^{\circ} \mathrm{C}$ and $14 \%$ of polyDMA from preceding DMA photopolymerization. The associated DMA concentration is $c_{\text {DMA }}=3.4 \mathrm{~mol} \cdot \mathrm{L}^{-1}, c_{\text {MMMP }}=25.5 \cdot 10^{-3} \mathrm{~mol} \cdot \mathrm{L}^{-1}$. The $k_{\mathrm{p}}$ value from PLP-SEC for the above conditions is $256 \mathrm{~L} \cdot \mathrm{mol}^{-1} \cdot \mathrm{s}^{-1}$.

The signal-to-noise quality that may be reached with the currently available experimental setup is not yet sufficient for obtaining high-quality first-derivative curves of $c_{\mathrm{R}}(t)$ data. So, the first-derivative strategy based on Eq. 3.5 was not applied. Instead, integrated forms of Eq. 3.5 were fitted to the experimental data in order to obtain termination rate coefficients.

According to Eq. 3.6, $c_{\mathrm{R}}(t)$ data may be analyzed by plotting the reciprocal of radical concentration versus time. If $k_{\mathrm{t}}$ remains constant and thus is independent of chain length, such a plot will yield a straight line which should intersect the ordinate at $c_{\mathrm{R}}(t) / c_{\mathrm{R}}{ }^{0}=1 . k_{\mathrm{t}}$ can be obtained from the slope of this straight line provided that ${c_{\mathrm{R}}}^{0}$ is known. The insert in Fig. 6.3 shows such a $c_{\mathrm{R}}{ }^{0} / c_{\mathrm{R}}(t)$ versus $t$ plot. No satisfactory linear fit can be obtained, which suggests that $k_{\mathrm{t}}$ is chain-length dependent. This observation is in full agreement with the now generally accepted picture [1]. 
A simple and frequently used way of taking the chain-length dependence of $k_{\mathrm{t}}$ into account is by employing the power-law model (Eq. 6.2) for termination of two macroradicals of identical chain length $i$. Substitution of this power-law expression into Eq. 3.5 and subsequent integration yields Eq. 6.4:

$$
\frac{c_{\mathrm{R}}(t)}{c_{\mathrm{R}}^{0}}=\left(\frac{2 \cdot k_{\mathrm{t}}^{0} \cdot c_{\mathrm{R}}^{0} \cdot t_{\mathrm{p}}^{\alpha}}{1-\alpha} \cdot t^{(1-\alpha)}+1\right)^{-1}
$$

where $t_{\mathrm{p}}=\left(k_{\mathrm{p}} \cdot c_{\mathrm{M}}\right)^{-1}$ is the so-called propagation time, that is, the time interval between two successive propagation steps. The dashed line in Fig. 6.3 represents the fit of Eq. 6.4 to the $c_{\mathrm{R}}(t) / c_{\mathrm{R}}{ }^{0}$ data. The fit is rather satisfactory with the exception of the early time regions, $0<t<0.3 \mathrm{~s}$. The parameters deduced from fitting Eq. 6.4 to the DMA data within the chainlength interval $1<i<1000$ are $\alpha=0.34 \pm 0.05$. With pulse-induced initial radical concentrations, $c_{\mathrm{R}}{ }^{0}$, of about $5 \cdot 10^{-6} \mathrm{~mol} \cdot \mathrm{L}^{-1}$ being obtained by ESR calibration, according to the procedure described in Experimental Part, and with the $k_{\mathrm{p}}$ and $c_{\mathrm{M}}$ values given in the legend to Fig. $6.3, k_{\mathrm{t}}^{0}$ is found to be $(3.8 \pm 0.7) \cdot 10^{6} \mathrm{~L} \cdot \mathrm{mol}^{-1} \cdot \mathrm{s}^{-1}$. From the SP-PLP-ESR signals measured at other conversions, $\alpha$ and $k_{\mathrm{t}}^{0}$ values have been found that agree with the values given above within the limits of experimental accuracy. The literature value for $k_{\mathrm{t}}^{0}$ from SPPLP-NIR experiments for DMA carried out at $40^{\circ} \mathrm{C}$ and 1000 bar is: $k_{\mathrm{t}}^{0}=(4.0 \pm 0.6) \cdot 10^{6} \mathrm{~L} \cdot \mathrm{mol}^{-1} \cdot \mathrm{s}^{-1}$ which, after extrapolation to $0{ }^{\circ} \mathrm{C}$ and to ambient pressure using the measured activation volume (for $<k_{\mathrm{t}}>$ ) of $10.8 \mathrm{~cm}^{3} \cdot \mathrm{mol}^{-1}$ and the estimated activation energy of $E_{\mathrm{A}}\left(k_{\mathrm{t}}\right)=17 \mathrm{~kJ} \cdot \mathrm{mol}^{-1}$, yields $k_{\mathrm{t}}^{0}=(2.3 \pm 0.8) \cdot 10^{6} \mathrm{~L} \cdot \mathrm{mol}^{-1} \cdot \mathrm{s}^{-1}$. The value of $18.7 \mathrm{~kJ} \cdot \mathrm{mol}^{-1}$ for the activation energy obtained at 2000 bar for termination of butyl methacrylate was reported [24], which is higher than reported earlier values lying between 3 and $8 \mathrm{~kJ} \cdot \mathrm{mol}^{-1}$ [37]. In the literature [25] were presented the experimental activation energies for dibutyl itaconate $E_{\mathrm{A}}\left(<k_{\mathrm{t}}>\right)=23 \mathrm{~kJ} \cdot \mathrm{mol}^{-1}$ and $E_{\mathrm{A}}\left(k_{\mathrm{t}}^{0}\right)=27.6 \mathrm{~kJ} \cdot \mathrm{mol}^{-1}$. On the other hand, an even higher activation energy value of $40.7 \mathrm{~kJ} \cdot \mathrm{mol}^{-1}$ has been reported by Vana et al. [26] for dicyclohexyl itaconate bulk polymerization. Therefore the value of $E_{\mathrm{A}}\left(k_{\mathrm{t}}\right)=17 \mathrm{~kJ} \cdot \mathrm{mol}^{-1}$ for DMA seems to be reasonable.

The agreement between the $k_{\mathrm{t}}^{0}$ values from the two independent SP-PLP techniques is satisfactory. Moreover, it needs to be noted that the $\alpha$ value from SP-PLP-ESR is higher than the SP-PLP-NIR value of $\alpha=0.15 \pm 0.04$ [27]. This is probably due to the fact that the NIR technique, which is applied at higher temperatures, primarily catches the chain-length dependence of $k_{\mathrm{t}}$ for larger radicals, which is weaker than the one for small radicals. 
As has been mentioned above, the quality of fits of Eq. 6.4 to $c_{\mathrm{R}}(t) / c_{\mathrm{R}}{ }^{0}$ data is insufficient at low $t$ (at $t<0.3 \mathrm{~s}$ ), which suggests that a more refined model for the chain-length dependence of $k_{\mathrm{t}}$ should be used. In Eq. $6.4 k_{\mathrm{t}}^{0}$ refers to the termination of radicals with chain length unity. On the other hand, the power-law dependence was derived for large coiled radicals [28]. Thus, $k_{\mathrm{t}}^{0}$ characterizes the termination behavior of a fictitious species which is large and coiled but of chain length $i=1$. It comes as no surprise that $k_{\mathrm{t}}^{0}$, which is extrapolated from the data obtained for macroradicals, does not coincide with the experimentally determined radical-radical combination rate coefficients for small radicals. This problem has recently been addressed by Smith et al. [10] who proposed to use a "composite model" (presented in section 6.1 ) with the theory-based value of $\alpha=0.16$ for long-chain radicals $[18,19,28]$ and with $\alpha$ being larger, e.g., around $\alpha=0.5$ [20,29], for small-chain radicals. The authors assumed that the transition between the regions of smaller and larger $\alpha$ occurs at around $i=100$. Indications for composite-type $k_{\mathrm{t}}$ models have already been seen in the experimental studies by de Kock [30] and by Vana et al. [31].

In order to test whether the kinetic data from SP-PLP-ESR are consistent with such a composite model, the following rearranged form of Eq. 6.4 may be used:

$$
\frac{c_{\mathrm{R}}^{0}}{c_{\mathrm{R}}(t)}-1=C \cdot t^{(1-\alpha)}
$$

where $C=\left(2 \cdot k_{\mathrm{t}}^{0} \cdot c_{\mathrm{R}}^{0} \cdot t_{\mathrm{p}}^{\alpha}\right) /(1-\alpha)$ if the termination kinetics follows the power law with a single exponent $\alpha$ from the very beginning, that is, from $t=0$ on. Plotting $\log \left(c_{\mathrm{R}}^{0} / c_{\mathrm{R}}(t)-1\right)$ vs. $\log (t)$ in this case should yield a straight line with the slope $1-\alpha$. For the two-exponent composite model proposed in reference [10], there should be two linear regions with slopes $1-\alpha_{1}$ (for $t<t_{\mathrm{c}}$, where $t_{\mathrm{c}}$ refers to the cross-over chain length from $\alpha_{1}$ to $\alpha_{2}$ behavior) and $1-\alpha_{2}$ (for $t>t_{\mathrm{c}}$, where simulation indicates that an almost accurate $\alpha_{2}$ value is obtained by fitting Eq. 6.5 to the data within the entire range of $t>t_{\mathrm{c}}$ ). Fig. 6.4 shows such a double logarithmic plot for the DMA data shown in Fig. 6.3. On the abscissa, $\log i$ is plotted as well as $\log t$. The two variables are proportional to each other by the equation $i=k_{\mathrm{p}} \cdot c_{\mathrm{M}} \cdot t$. In the present work, the dependence of $k_{\mathrm{p}}$ on chain length will be restricted to lower values up to $i=10$. It seems to be justified to use a constant $k_{\mathrm{p}}$ for modeling the extended chain length up to $i=1000$. It should be noted that the chain length interval $1<i<10$ was difficult to analyze because a scattering of the experimental data points causes a large uncertainty in the double-log plot in the initial time region. 


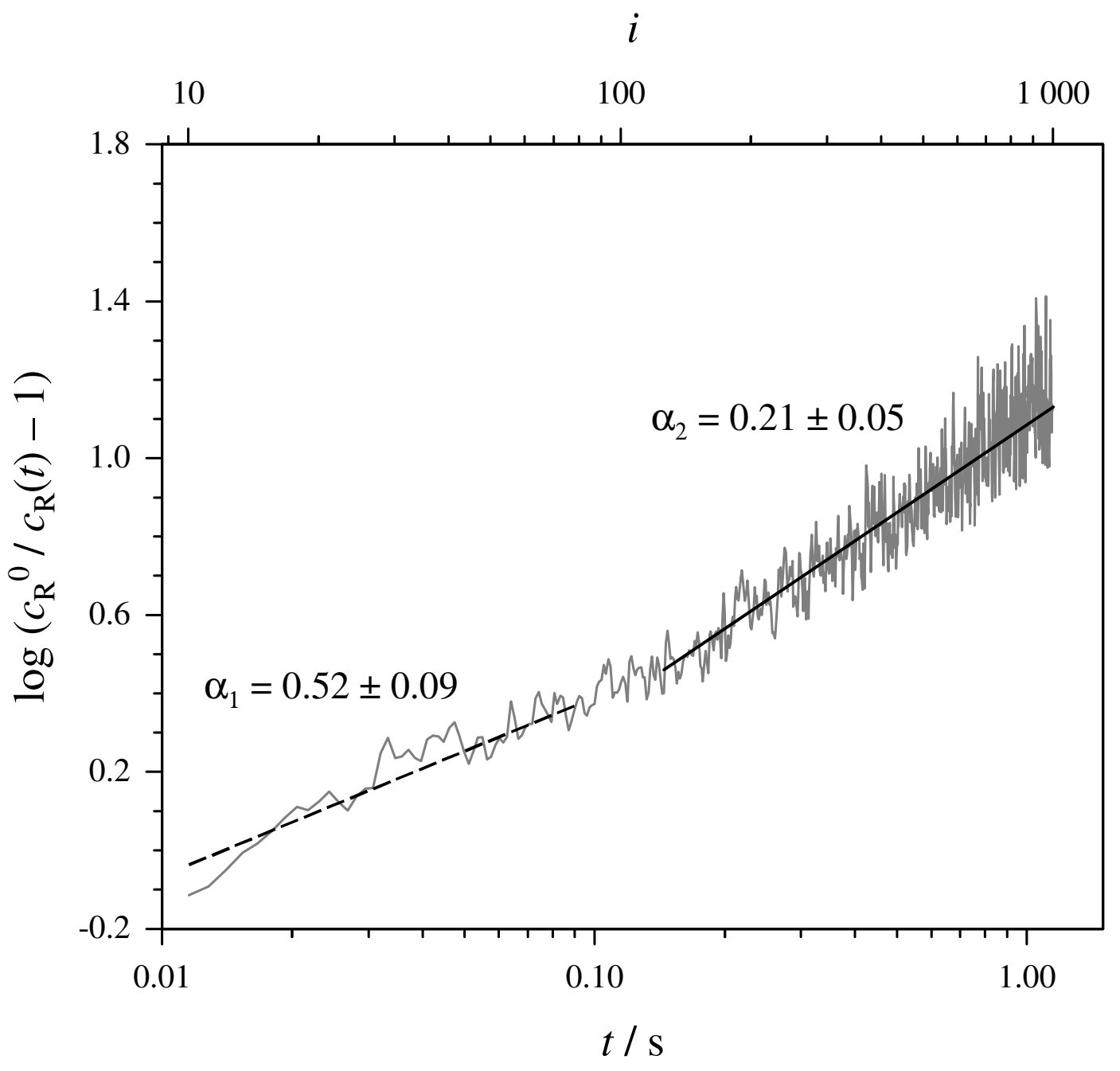

Fig. 6.4 Double-log plot, according to Eq. 6.5, of the DMA trace from Fig. 6.3. The straight lines are best linear fits within the chain-length intervals $10<i<100$ ( --- ) and $100<i<1000$ ( - ) , respectively.

The data plotted in Fig. 6.4 clearly shows that a single straight line cannot be adequately fitted to the data within the entire chain-length region, whereas two straight lines provide a very satisfactory representation. The line fitted to the data at low $i$ has a weaker slope, which is associated with a larger value of $\alpha$.The opposite is true for the data at higher $i$. The precise location of the cross-over point between the regions of high and low $\alpha$ is difficult to identify. This is most likely due to the fact that the transition is not at all sharp. As Fig. 6.4 suggests, the assumption that such a transition occurs at $i=100$, as proposed by Smith et al. [10], is a very reasonable one. The chain-length interval $0<i<1000$ was subdivided into two chainlength intervals, below and above $i=100$. The best linear fits to the data within each of these two chain-length intervals are shown in Fig. 6.4. The exponents $\alpha_{1}$ and $\alpha_{2}$, calculated from the slopes of the lines, are $0.52 \pm 0.09$ and $0.21 \pm 0.05$ for the low $i$ and high $i$ regions, 
respectively. The same procedure was applied for fitting the entire set of experimental SPPLP-ESR traces obtained for DMA bulk polymerizations at $0{ }^{\circ} \mathrm{C}$, ambient pressure, monomer conversions up to 30 per cent, and for three MMMP photoinitiator concentrations, $6.3 \cdot 10^{-3} \mathrm{~mol} \cdot \mathrm{L}^{-1}, 9.6 \cdot 10^{-3} \mathrm{~mol} \cdot \mathrm{L}^{-1}$ and $25.5 \cdot 10^{-3} \mathrm{~mol} \cdot \mathrm{L}^{-1}$. The resulting $\alpha_{1}$ and $\alpha_{2}$ values are plotted against monomer conversion, $X$, in Fig. 6.5 .

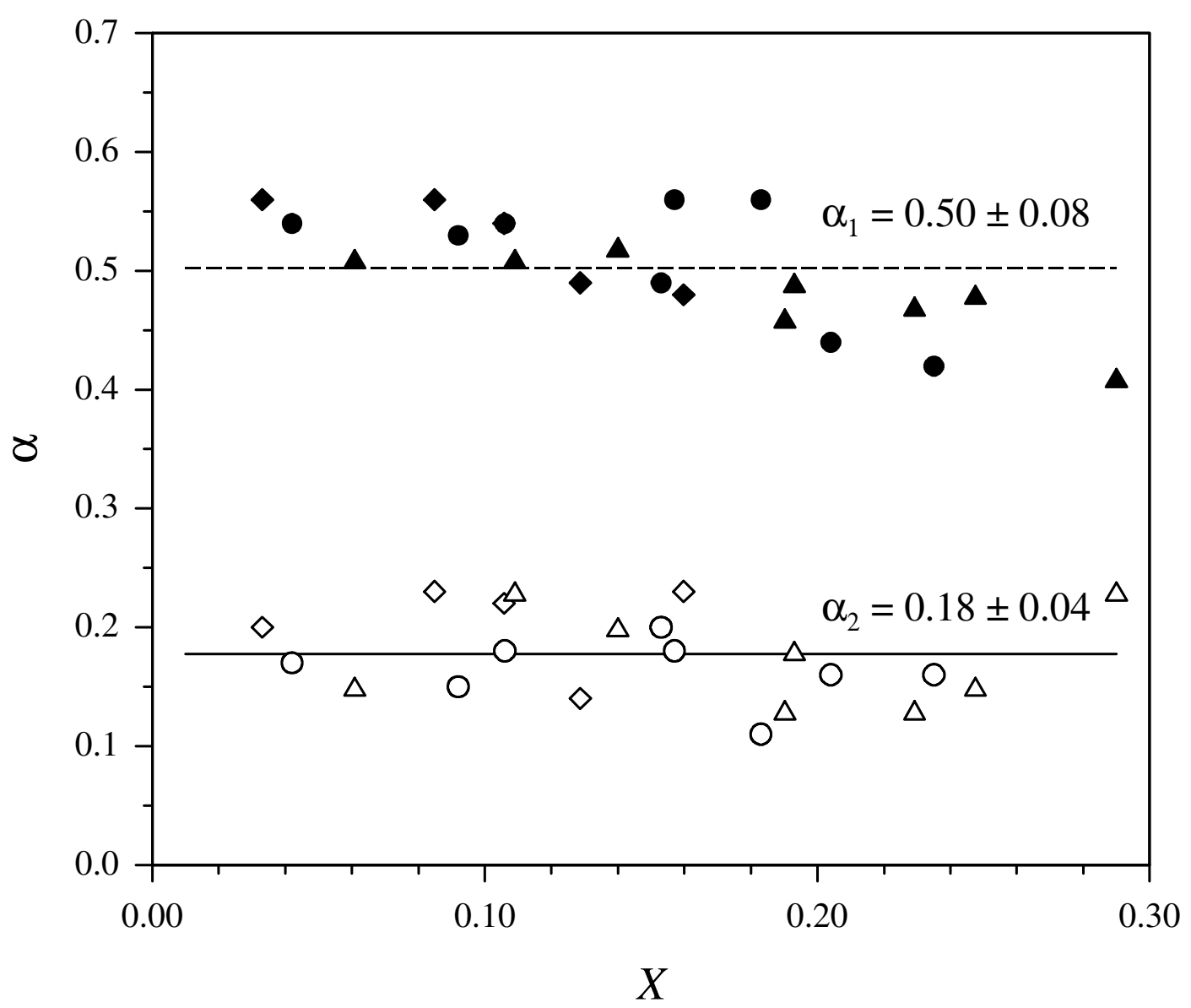

Fig. 6.5 Conversion $(X)$ dependence of the power-law exponent $\alpha$ for DMA bulk homopolymerization at $0{ }^{\circ} \mathrm{C}$ measured via SP-PLP-ESR with MMMP used as the photo-initiator. The experiments were carried out at three initial MMMP concentrations: $6.3 \cdot 10^{-3} \mathrm{~mol} \cdot \mathrm{L}^{-1}$ (diamonds), $9.6 \cdot 10^{-3} \mathrm{~mol} \cdot \mathrm{L}^{-1}$ (circles) and $25.5 \cdot 10^{-3} \mathrm{~mol} \cdot \mathrm{L}^{-1}$ (triangles). The straight lines represent the arithmetic mean values of the low $i$ ( --- ) and high $i(-)$ for the monomer conversion range under investigation.

Although there may be a weak dependence on conversion, arithmetic mean values for the conversion range under investigation were calculated: $\alpha_{1}=0.50 \pm 0.08$ and $\alpha_{2}=0.18 \pm 0.04$. These results are in remarkably good agreement with the composite model of Smith et al. 
[10], in that bi-exponential behavior, above and below $i=100$, is seen with $\alpha_{2}=0.16$ and with $\alpha_{1}$ being significantly larger, around 0.5. Fig. 6.5 illustrates that the novel SP-PLP-ESR method allows $k_{\mathrm{t}}(i, i)$ to be investigated over a wide chain-length region, covering the ranges of high and low $\alpha$. The new SP-PLP-ESR technique therefore provides access to a detailed analysis of $k_{\mathrm{t}}$ as a function of chain length and of the degree of monomer conversion. Correlation between the $k_{\mathrm{t}}$ data for macroradicals and the radical-radical reaction rate coefficients for small radicals, according to the lines of arguments of reference [10], appears to be particularly attractive.

\subsubsection{DEPENDENCE OF $\boldsymbol{k}_{\mathrm{t}}(\mathrm{CHMA})$ ON MONOMER CONVERSION}

Fig. 6.6 shows a typical 13-line ESR spectrum for low-conversion CHMA polymerization.

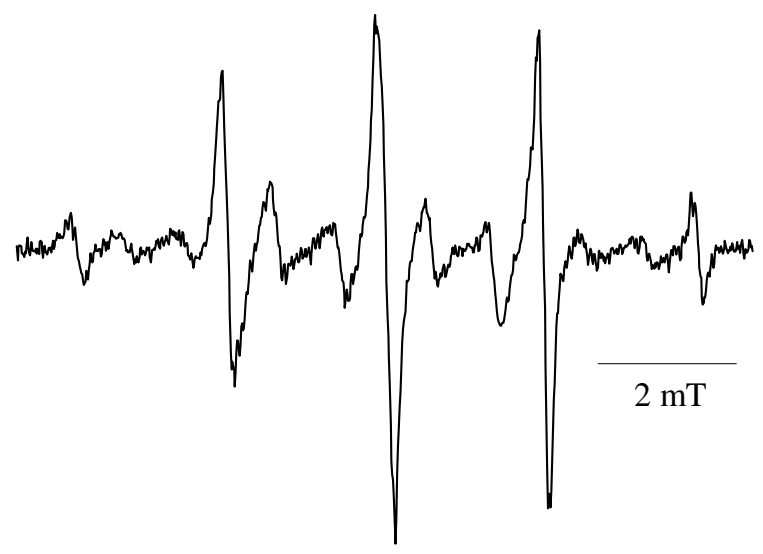

Fig. 6.6 Spectrum of CHMA macroradical from quasistationary polymerization initiated by laser pulses applied at a repetition rates of $20 \mathrm{~Hz}$ and carried out at $0{ }^{\circ} \mathrm{C}, c_{\text {MMMP }}=20.8 \cdot 10^{-3} \mathrm{~mol} \cdot \mathrm{L}^{-1}$.

Experimental results were obtained in the same way as described in section 6.1.1 for DMA. As follows from Eq. 3.6, experimental $c_{\mathrm{R}}(t)$ data can be analyzed by plotting the reciprocal of radical concentration vs. time. If $k_{\mathrm{t}}$ were constant, such a plot would give a straight line passing through unity at $t=0 . k_{\mathrm{t}}$ could then be calculated from the slope of this line. Fig. 6.7 (upper part) shows such a plot for CHMA. The straight line is the best linear fit to the data. As can be seen, the experimental $c_{\mathrm{R}}{ }^{0} / c_{\mathrm{R}}(t)$ curve clearly deviates from the best linear fit, which indicates that $k_{\mathrm{t}}$ for this monomer is chain-length dependent. 


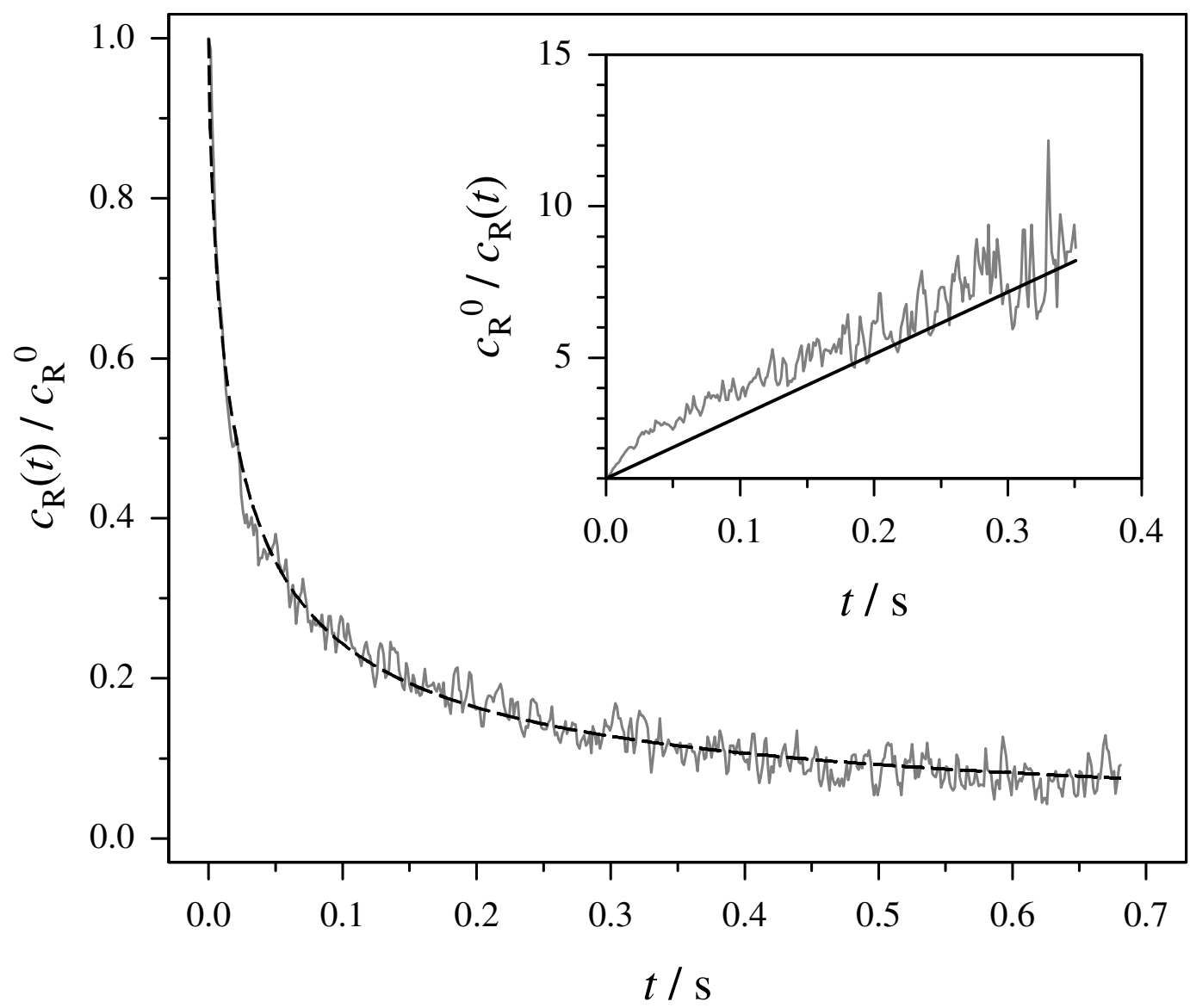

Fig. 6.7 Decay in radical concentration after applying a laser pulse at $t=0$ as measured by ESR for a CHMA bulk polymerization at $0{ }^{\circ} \mathrm{C}$ and $14 \%$ of polyCHMA from preceding CHMA photopolymerization. The associated CHMA concentration is $c_{\mathrm{CHMA}}=5.8 \mathrm{~mol} \cdot \mathrm{L}^{-1}, c_{\mathrm{MMMP}}=20.8 \cdot 10^{-3} \mathrm{~mol} \cdot \mathrm{L}^{-1}$. The $k_{\mathrm{p}}$ value from PLP-SEC for the above conditions is $250 \mathrm{~L} \cdot \mathrm{mol}^{-1} \cdot \mathrm{s}^{-1}[38]$.

The dashed line in Fig. 6.7 represents the fit of Eq. 6.4 to the $c_{\mathrm{R}}(t) / c_{\mathrm{R}}{ }^{0}$ data. The fit is rather satisfactory with the exception of the early time regions, $0<t<0.2 \mathrm{~s}$. The parameters deduced from fitting Eq. 6.4 to the data within the chain-length interval $1<i<1000$ are: $\alpha=0.32 \pm 0.05$. With pulse-induced initial radical concentrations, $c_{\mathrm{R}}{ }^{0}$, of about $1 \cdot 10^{-6} \mathrm{~mol} \cdot \mathrm{L}^{-1}$ being obtained by ESR calibration, according to the procedure described in Experimental Part, and with the $k_{\mathrm{p}}$ and $c_{\mathrm{M}}$ values given in the legend to Fig. $6.7, k_{\mathrm{t}}^{0}$ is found to be $(7.6 \pm 0.7) \cdot 10^{6} \mathrm{~L} \cdot \mathrm{mol}^{-1} \cdot \mathrm{s}^{-1}$.

Fig. 6.8 presents the double logarithmic plot for the CHMA data shown in Fig. 6.7 according to Eq. 6.5. On the abscissa, $\log i$ is plotted as well as $\log t$. The two variables are correlated with each other by following equation $i=k_{\mathrm{p}} \cdot c_{\mathrm{M}} \cdot t$. 


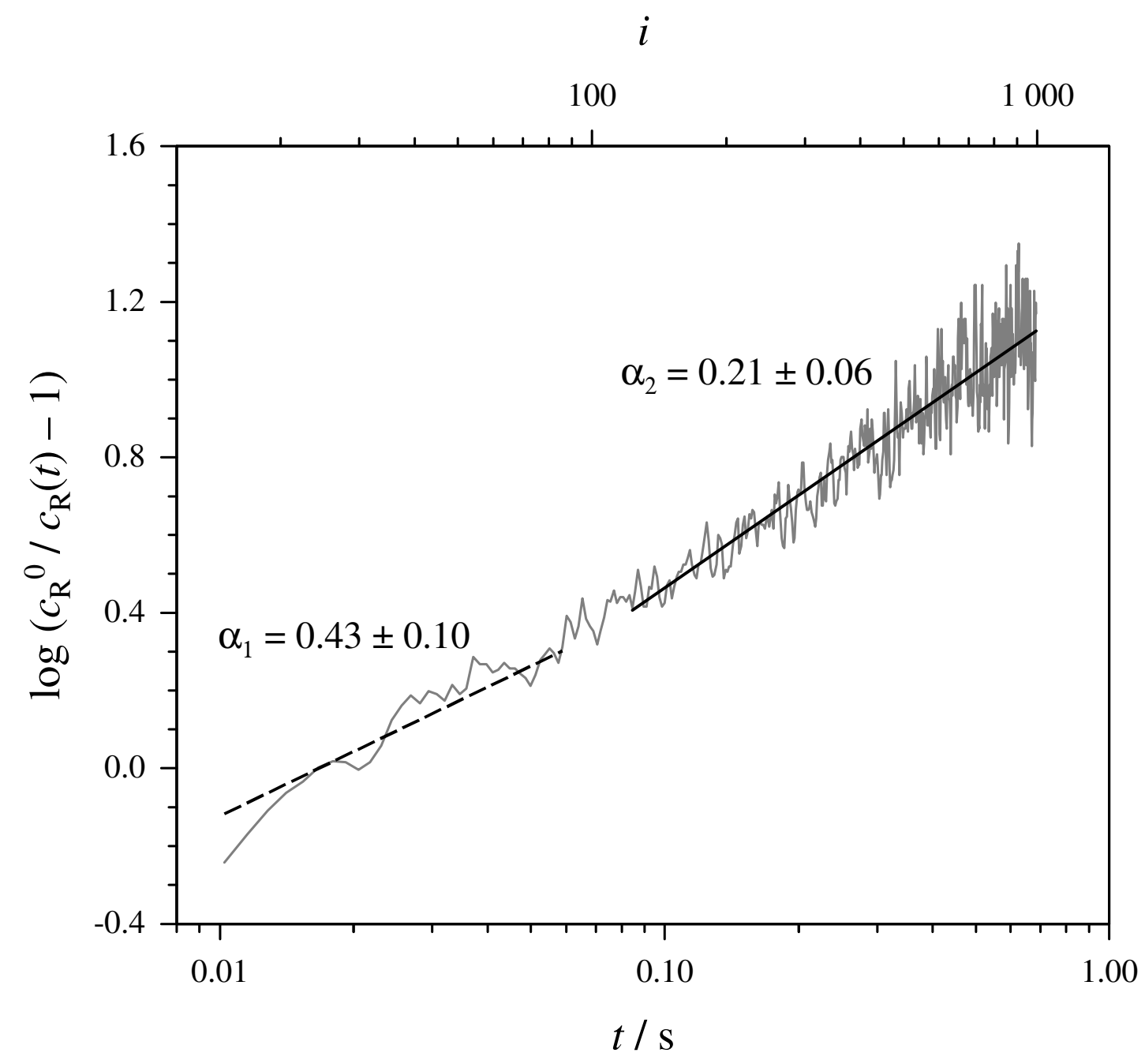

Fig. 6.8 Double-log plot, according to Eq. 6.5, of the CHMA trace. The straight lines are best linear fits within the chain-length intervals $15<i<100(---)$ and $100<i<1000$ ( - ) , respectively.

It is difficult to conclude from Fig. 6.8 where the transition in the chain-length dependence of $k_{\mathrm{t}}$, characterized by a change in $\alpha$, occurs. For this reason, it was assumed that such a transition occurs at about $i=100$. The chain-length interval of $1<i<1000$ was divided into two chain-length intervals, below and above $i=100$. The best linear fits to the data within each of these two chain-length intervals are shown in Fig. 6.8. The values of the exponents $\alpha_{1}$ and $\alpha_{2}$, calculated from the slopes, are $0.43 \pm 0.10$ and $0.21 \pm 0.06$, respectively. Following the above procedure all the other experimental signals for CHMA were analyzed. Fig. 6.9 shows calculated $\alpha_{1}$ and $\alpha_{2}$ values plotted against monomer conversion, $X$, for CHMA homopolymerization in bulk at $0{ }^{\circ} \mathrm{C}$. 


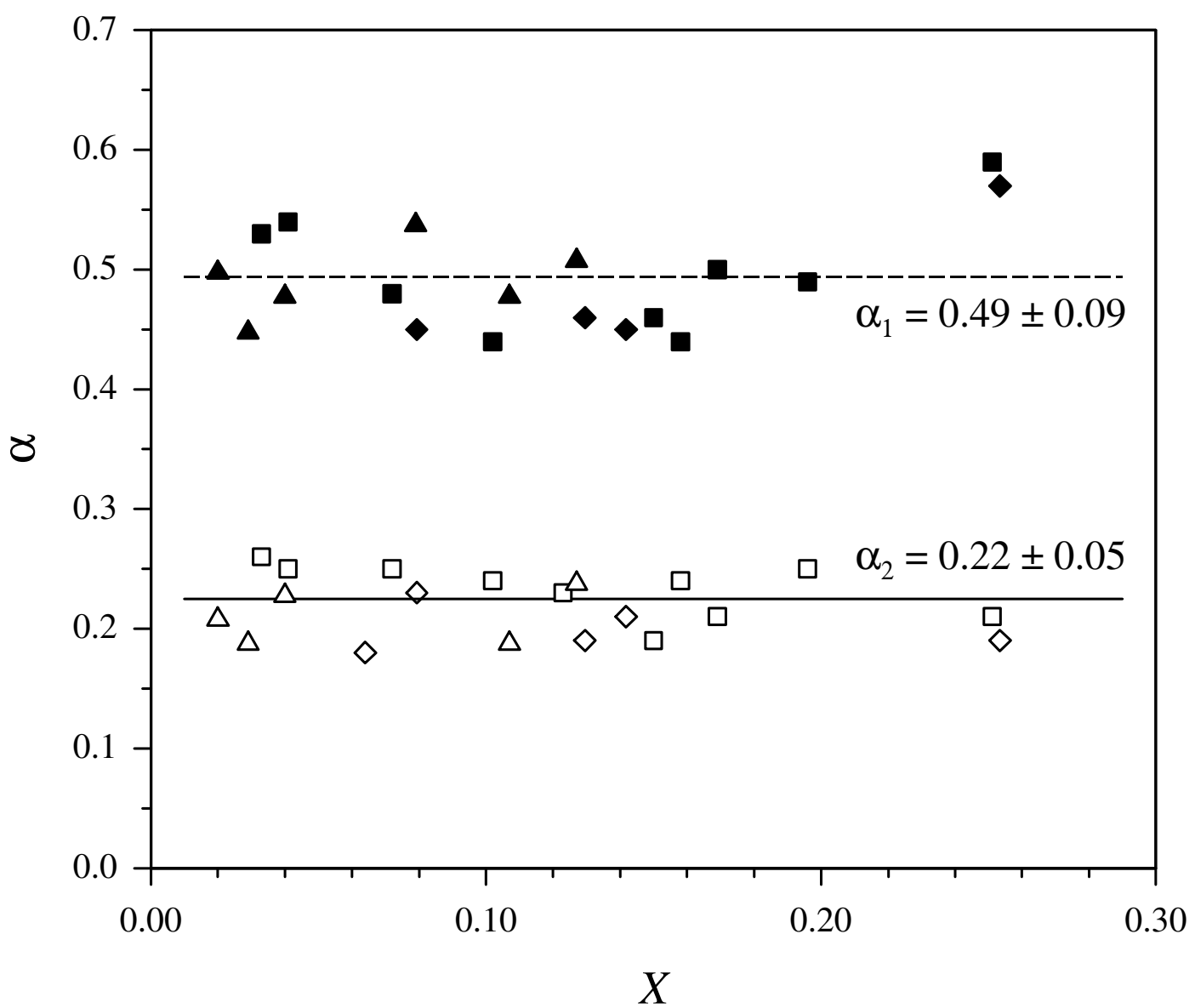

Fig. 6.9 Conversion $(X)$ dependence of the power-law exponent $\alpha$ for CHMA bulk homo-polymerization at $0{ }^{\circ} \mathrm{C}$ measured via SP-PLP-ESR with MMMP being used as the photoinitiator. The experiments were carried out at three initial MMMP concentrations: $9.7 \cdot 10^{-3} \mathrm{~mol} \cdot \mathrm{L}^{-1}$ (triangles), $16.5 \cdot 10^{-3} \mathrm{~mol} \cdot \mathrm{L}^{-1}$ (squares) and $20.8 \cdot 10^{-3} \mathrm{~mol} \cdot \mathrm{L}^{-1}$ (diamonds). The straight lines represent the arithmetic mean values of the low $i$ ( --- ) and high $i$ ( - ) for the monomer conversion range under investigation.

Arithmetic mean values for the conversion range under investigation were calculated: $\alpha_{1}=0.49 \pm 0.09$ and $\alpha_{2}=0.22 \pm 0.05$. Although the values of $\alpha$ are slightly different from the ones obtained for DMA, these results are in good agreement with the composite model of Smith et al. [10] in that bi-exponential behavior, above and below $i=100$, is seen. 


\subsubsection{CONVERSION AND TEMPERATURE DEPENDENCE OF $\boldsymbol{k}_{\mathrm{t}}(\mathrm{BZMA})$}

Fig. 6.10 shows typical 13-line ESR spectra for low-conversion BzMA polymerization at different temperatures.

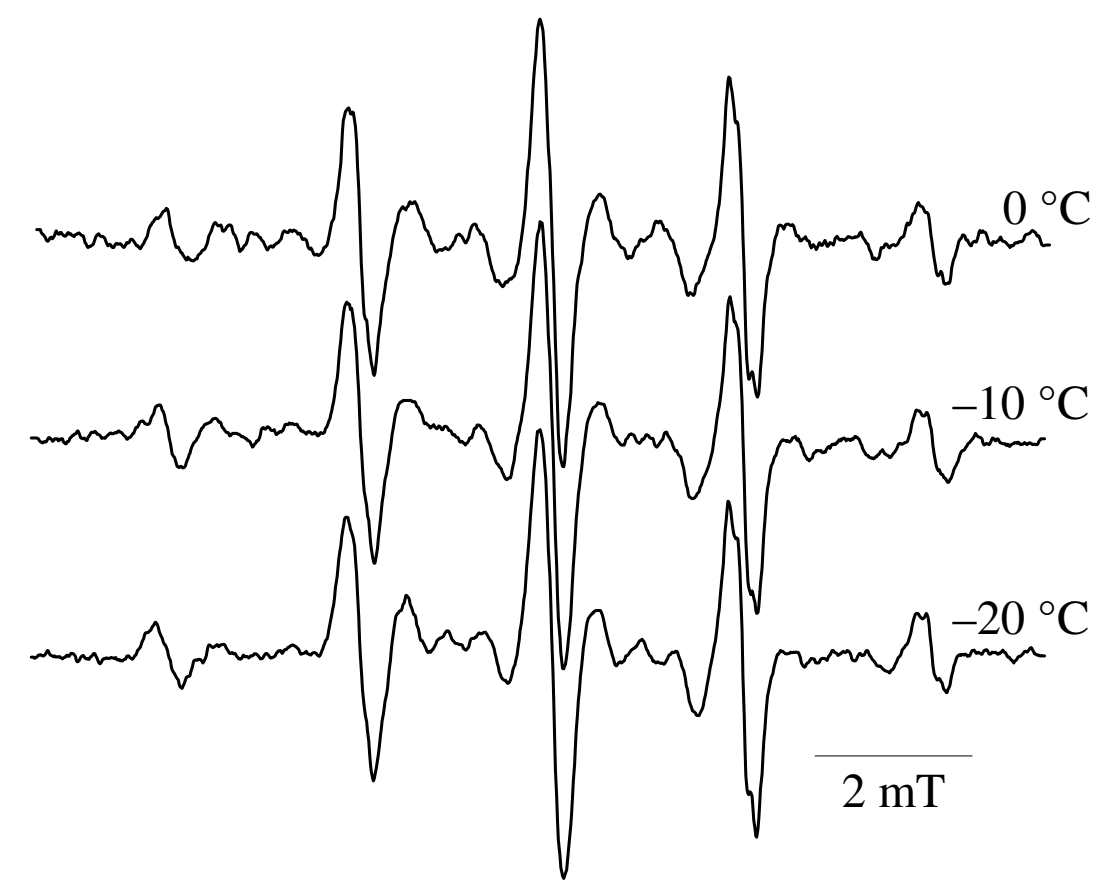

Fig. 6.10 Spectra of BzMA macroradicals from quasistationary polymerization initiated by laser pulses applied at a repetition rates of $20 \mathrm{~Hz}$ and carried out at $0{ }^{\circ} \mathrm{C},-10{ }^{\circ} \mathrm{C}$ and $-20{ }^{\circ} \mathrm{C}, c_{\mathrm{MMMP}}=22.9 \cdot 10^{-3} \mathrm{~mol} \cdot \mathrm{L}^{-1}$.

As already pointed out in the Experimental Section, the new SP-PLP-ESR technique is particularly well suited for monomers with relatively low propagation and termination rate coefficients. For the monomers under investigation at temperatures higher than $0{ }^{\circ} \mathrm{C}$, a time resolution of $1 \mathrm{~ms}$ is insufficient for quantitative characterization of termination kinetics because of the high values of $k_{\mathrm{p}}$. BzMA was chosen for investigations into termination kinetics because of its low melting point, which allows for experiments below $0{ }^{\circ} \mathrm{C}$.

Decays in radical concentrations were analyzed using the power-law model with one exponent (Eq. 6.4). Fig. 6.11 shows the fit of Eq. 6.4 to the $c_{\mathrm{R}}(t) / c_{\mathrm{R}}{ }^{0}$ data (dashed line). The parameters deduced from fitting Eq. 6.4 to the data within the chain-length interval $1<i<1000$ are: $\alpha=0.30 \pm 0.07$. Following the above procedure, all the other experimental signals for BzMA were analyzed (Tab. 6.1). With pulse-induced initial radical concentrations, $c_{\mathrm{R}}{ }^{0}$, of about $4 \cdot 10^{-6} \mathrm{~mol} \cdot \mathrm{L}^{-1}$ being obtained by ESR calibration, according to the procedure 
described above, and with the $k_{\mathrm{p}}$ and $c_{\mathrm{M}}$ values given in the legend to Fig. $6.11, k_{\mathrm{t}}^{0}$ at $0{ }^{\circ} \mathrm{C}$ is found to be $(1.3 \pm 0.8) \cdot 10^{7} \mathrm{~L} \cdot \mathrm{mol}^{-1} \cdot \mathrm{s}^{-1}$.

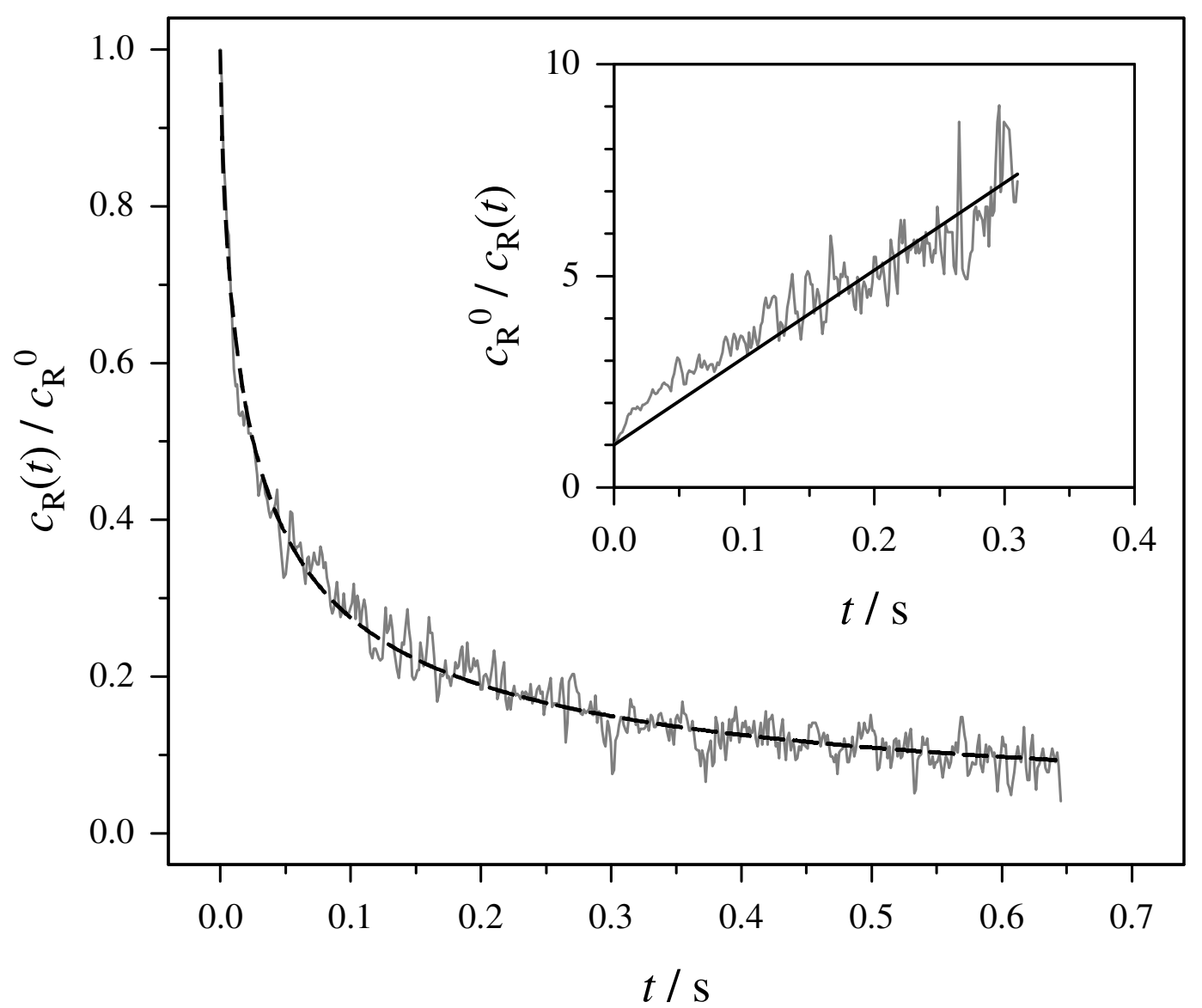

Fig. 6.11 Decay in radical concentration after applying a laser pulse at $t=0$ as measured by ESR for a BzMA bulk polymerization at $0{ }^{\circ} \mathrm{C}$ and $5 \%$ of polyBzMA from preceding BzMA photopolymerization. The associated BzMA concentration is $c_{\mathrm{BzMA}}=5.7 \mathrm{~mol} \cdot \mathrm{L}^{-1}$. The $k_{\mathrm{p}}$ value from PLP-SEC for the above conditions is $274 \mathrm{~L} \cdot \mathrm{mol}^{-1} \cdot \mathrm{s}^{-1}[38]$.

The insert in Fig. 6.11 shows a plot of the reciprocal of radical concentration vs. time. If $k_{\mathrm{t}}$ does not depend on chain length, such a plot will yield a straight line which should intersect the ordinate at $c_{\mathrm{R}}(t) / c_{\mathrm{R}}{ }^{0}=1$ according to Eq. 3.6 and $k_{\mathrm{t}}$ can be obtained from the slope of this straight line provided that $c_{\mathrm{R}}{ }^{0}$ is known. No satisfactory linear fit can be obtained, which indicates a chain-length dependence of $k_{\mathrm{t}}$.

Fig. 6.12 shows a double logarithmic plot for the BzMA data shown in Fig. 6.11 according to Eq. 6.5. On the abscissa, $\log i$ is plotted as well as $\log t$. 


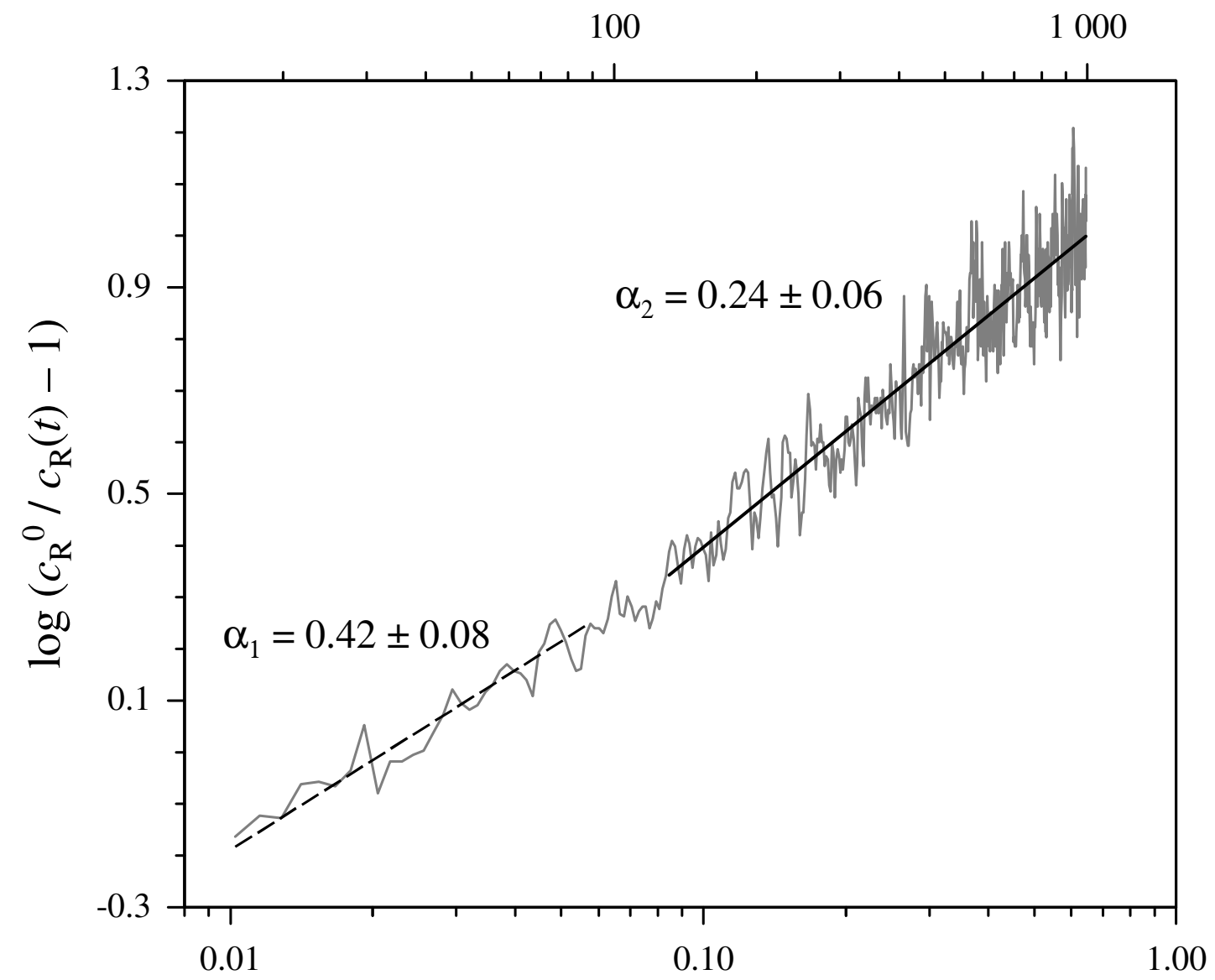

Fig. 6.12 Double-log plot, according to Eq. 6.3, of the BzMA trace. The straight lines are best linear fits within the chain-length intervals $15<i<100(---)$ and $100<i<1000$ ( - ) , respectively.

The double logarithmic plot of the experimental data given in Fig. 6.12 clearly shows that a single straight line cannot be adequately fitted to the data within the entire chain-length region, whereas two straight lines provide a very satisfactory representation. The line fitted to the data at low $i$ has a weaker slope, which is associated with a larger value of $\alpha$. The opposite is true for the data at higher $i$. The precise location of the crossover point between the regions of high and low $\alpha$ is difficult to identify. This is most likely due to the fact that the transition is not at all sharp. As Fig. 6.12 suggests, assuming that such a transition occurs at $i=100$, as proposed by Smith et al. [10], is a very reasonable choice. The entire chain-length interval $0<i<1000$ was subdivided into two chain-length intervals, below and above $i=100$. The best linear fits to the data within each of these two chain-length intervals are shown in Fig. 6.12. The exponents $\alpha_{1}$ and $\alpha_{2}$, calculated from the slopes of the lines, are $0.42 \pm 0.08$ and $0.24 \pm 0.06$ for the low $i$ and high $i$ regions, respectively. The same procedure was applied for fitting within the entire set of experimental SP-PLP-ESR traces obtained for BzMA bulk polymerizations at $0{ }^{\circ} \mathrm{C},-10{ }^{\circ} \mathrm{C}$, and $-20{ }^{\circ} \mathrm{C}$, ambient pressure, monomer conversions up to 
17 per cent, and for several MMMP photoinitiator concentrations. The resulting $\alpha_{1}$ and $\alpha_{2}$ values obtained at $0{ }^{\circ} \mathrm{C},-10{ }^{\circ} \mathrm{C}$, and $-20^{\circ} \mathrm{C}$ are plotted against monomer conversion, $X$, in Fig. 6.13, Fig. 6.14 and Fig. 6.15 respectively.

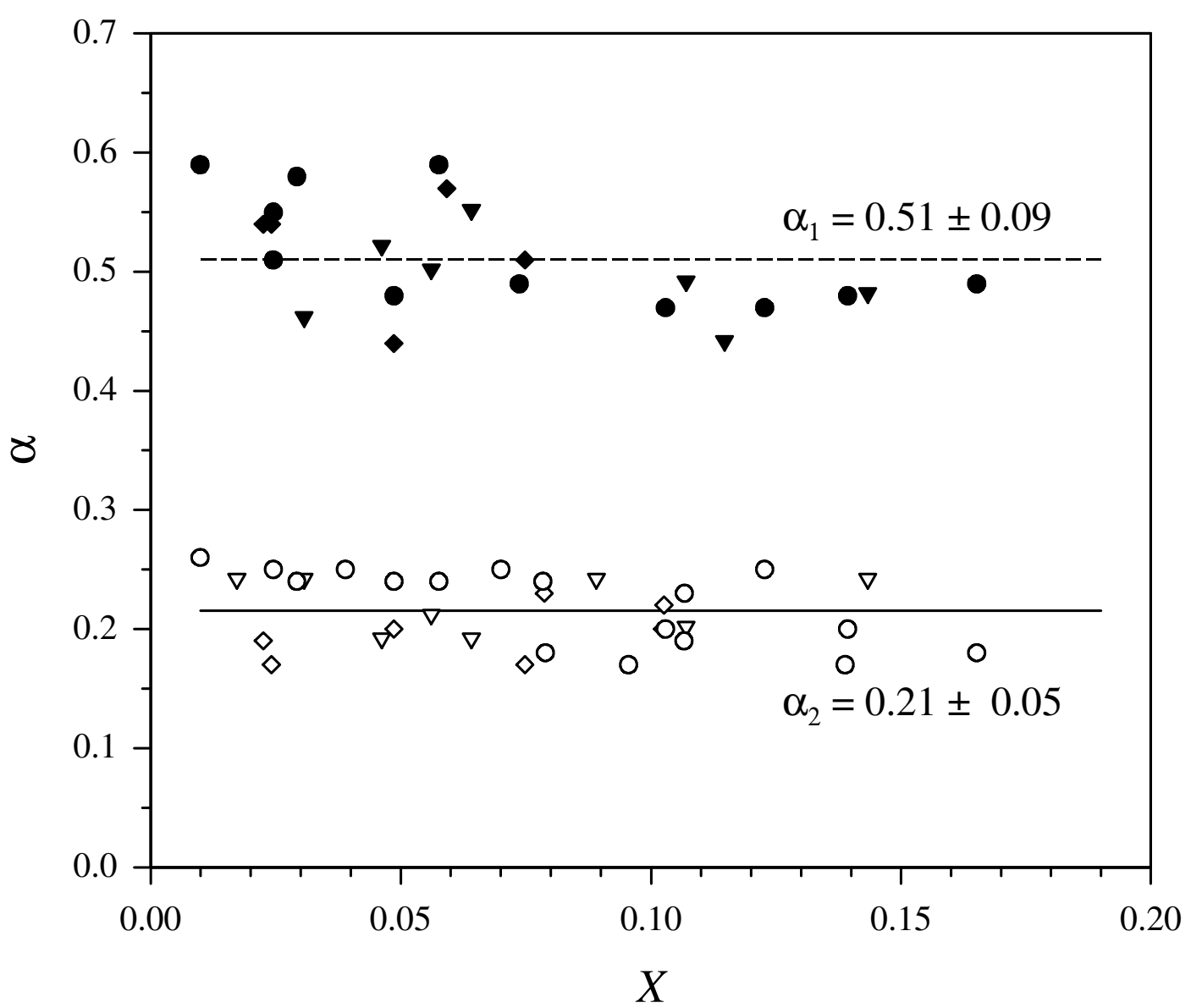

Fig. 6.13 Conversion $(X)$ dependence of the power-law exponent $\alpha$ for BzMA bulk homo-polymerization at $0{ }^{\circ} \mathrm{C}$ measured via SP-PLP-ESR with MMMP used as the photo-initiator. The experiments were carried out at three initial MMMP concentrations: $13.8 \cdot 10^{-3} \mathrm{~mol} \cdot \mathrm{L}^{-1}$ (diamonds), $17.4 \cdot 10^{-3} \mathrm{~mol} \cdot \mathrm{L}^{-1}$ (triangles), and $22.9 \cdot 10^{-3} \mathrm{~mol} \cdot \mathrm{L}^{-1}$ (circles). The straight lines represent the arithmetic mean values of the low $i$ ( --- ) and high $i(-)$ for the monomer conversion range under investigation. 


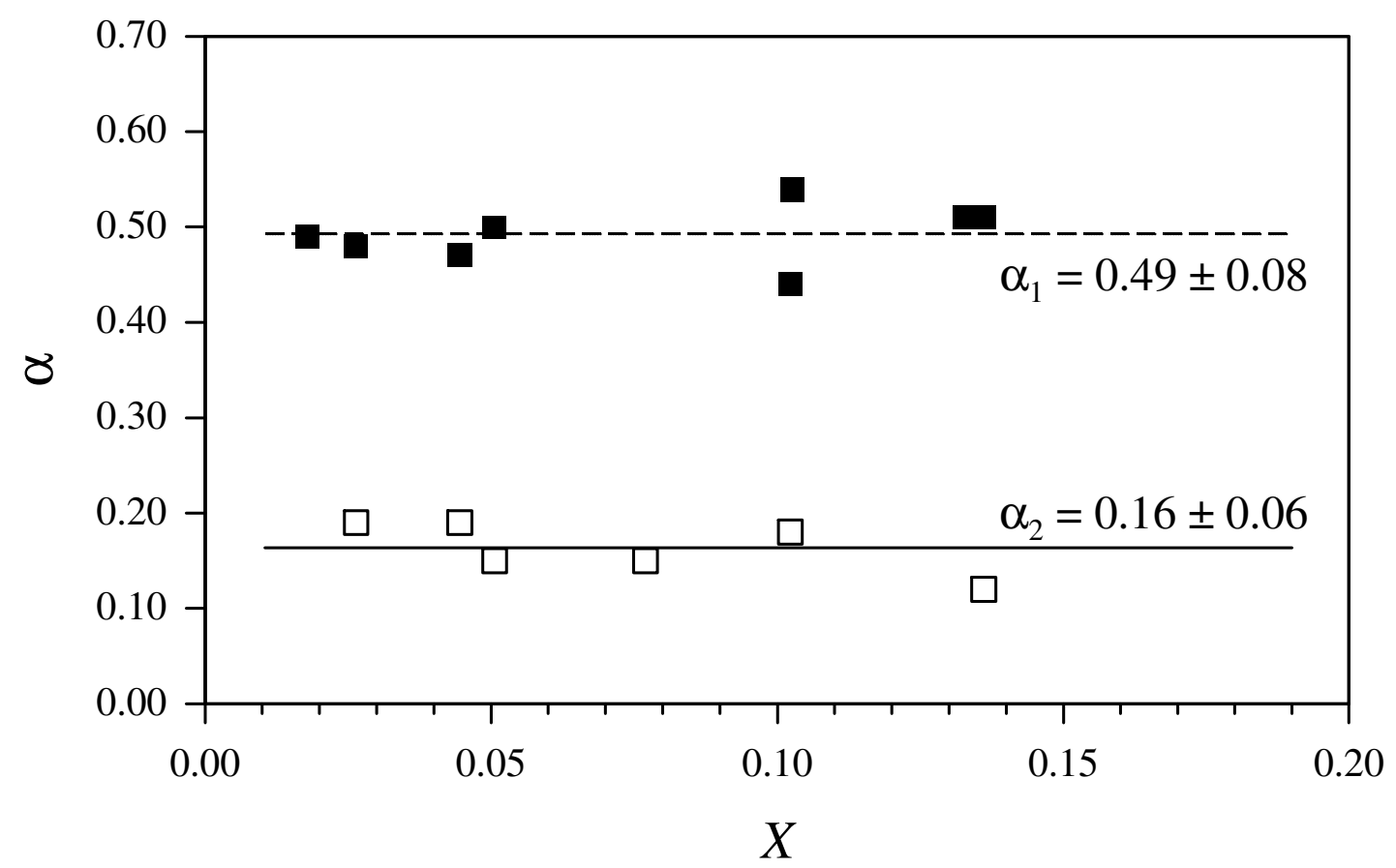

Fig. 6.14 Conversion $(X)$ dependence of the power-law exponent $\alpha$ for BzMA bulk homo-polymerization at $-10{ }^{\circ} \mathrm{C}$ measured via SP-PLP-ESR with MMMP used as the photo-initiator, $c_{\mathrm{MMMP}}=22.9 \cdot 10^{-3} \mathrm{~mol} \cdot \mathrm{L}^{-1}$ (squares). The straight lines represent the arithmetic mean values of the low $i(---)$ and high $i(-)$ for the monomer conversion range under investigation.

Although a weak dependence on conversion in Fig. 6.15 for $100<i<1000$ can be seen, arithmetic mean values for the conversion range under investigation were calculated (Tab. 6.1). These results are in good agreement with the composite model of Smith et al. [10] in that bi-exponential behavior is seen with $\alpha_{2}=0.16$ and with $\alpha_{1}$ being significantly larger, around 0.5 . The transition between both regions occurs at around $i=100$. 


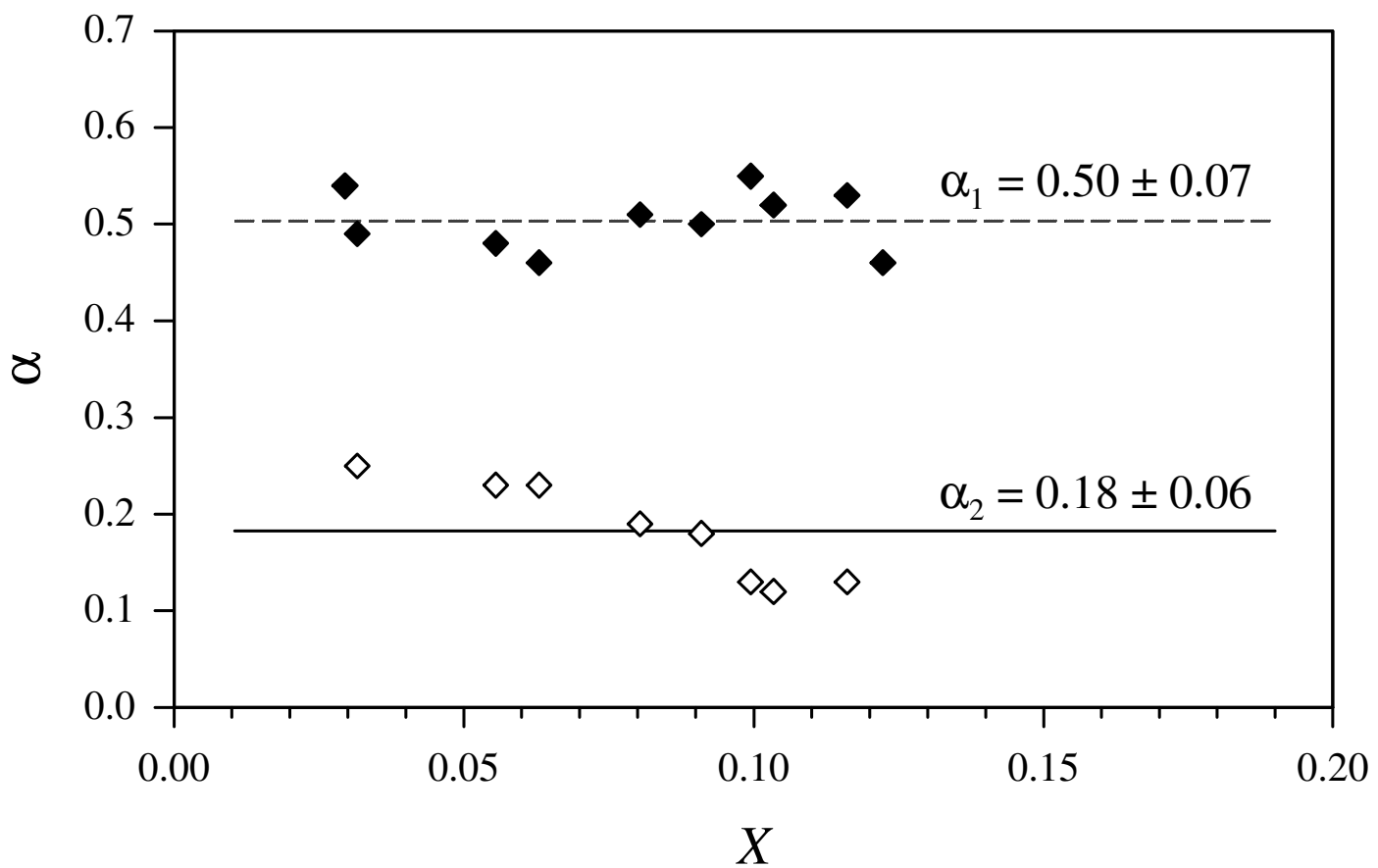

Fig. 6.15 Conversion $(X)$ dependence of the power-law exponent $\alpha$ for BzMA bulk homo-polymerization at $-20{ }^{\circ} \mathrm{C}$ measured via SP-PLP-ESR with MMMP used as the photo-initiator, $c_{\text {MMMP }}=22.9 \cdot 10^{-3} \mathrm{~mol} \cdot \mathrm{L}^{-1}$ (diamonds). The straight lines represent the arithmetic mean values of the low $i(---)$ and high $i(-)$ for the monomer conversion range under investigation.

Tab. 6.1 summarizes all rate coefficients obtained in this work for BzMA by SP-PLP-ESR; propagation rate coefficients were estimated from the data presented in Ref. [38].

\begin{tabular}{ccccccc}
\hline$\Theta /{ }^{\circ} \mathrm{C}$ & $\begin{array}{c}k_{\mathrm{p}} / \\
\mathrm{L} \cdot \mathrm{mol}^{-1} \cdot \mathrm{s}^{-1}\end{array}$ & $\begin{array}{c}<k_{\mathrm{t}}{ }^{*} 0<i<1000 \\
\mathrm{~L} \cdot \mathrm{mol}^{-1} \cdot \mathrm{s}^{-1}\end{array}$ & $\begin{array}{c}k_{\mathrm{t}}{ }^{* * *} / \\
\mathrm{L} \cdot \mathrm{mol}^{-1} \cdot \mathrm{s}^{-1}\end{array}$ & $\alpha^{* *}$ & $\begin{array}{c}\alpha_{1} \\
i<100\end{array}$ & $\begin{array}{c}\alpha_{2} \\
100<i<1000\end{array}$ \\
\hline 0 & 274 & $3.8 \cdot 10^{6}$ & $1.3 \cdot 10^{7}$ & 0.30 & 0.51 & 0.21 \\
-10 & 188 & $3.2 \cdot 10^{6}$ & $1.2 \cdot 10^{7}$ & 0.32 & 0.49 & 0.16 \\
-20 & 125 & $3.1 \cdot 10^{6}$ & $1.1 \cdot 10^{7}$ & 0.35 & 0.50 & 0.18 \\
\hline
\end{tabular}

All coefficients refer to ambient pressure

* obtained using equation for ideal kinetics (Eq. 3.6),

** obtained using Eq. 6.4

Tab. 6.1 Rate coefficients of BzMA bulk polymerization at low conversion (0 to $17 \%$ ).

Plotted in Fig. 6.16 is the temperature dependence of $<k_{\mathrm{t}}>_{0<i<1000}$ and $k_{\mathrm{t}}^{0}$ for BzMA homopolymerization at ambient pressure. The SP-PLP-ESR studies were performed between 
$-20{ }^{\circ} \mathrm{C}$ and $0{ }^{\circ} \mathrm{C}$ with the experimental temperature range being limited toward lower temperatures because of solidification and toward higher temperatures because of high $k_{\mathrm{p}}$ values and thus of insufficient ESR resolution.

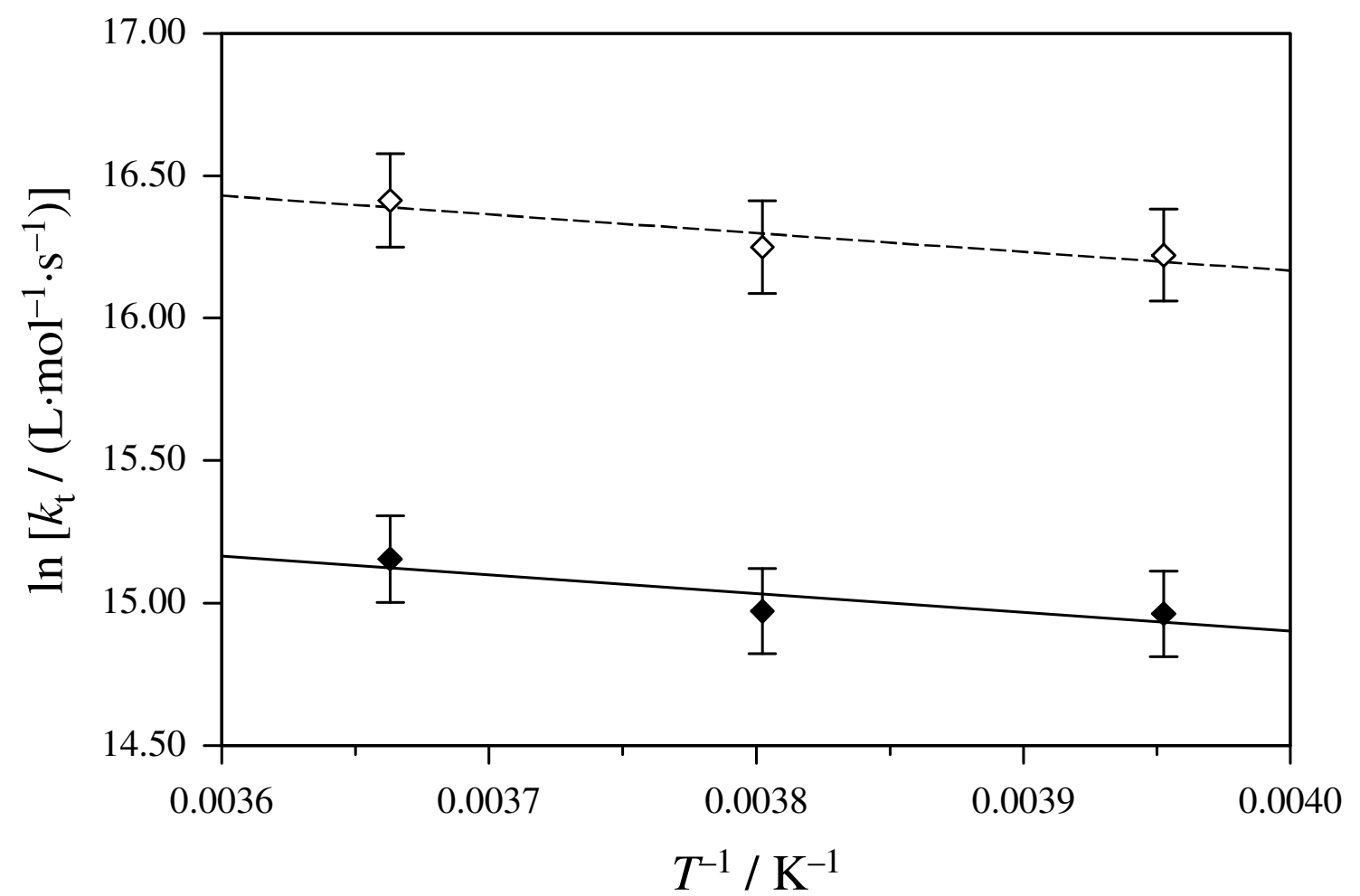

Fig. 6.16 Temperature dependence of the termination rate coefficient $<k_{\mathrm{t}}>_{0<i<1000}$, obtained via Eq. 3.6 for ideal kinetics (full symbols), and $k_{\mathrm{t}}{ }^{0}$, obtained via Eq. 6.4 (open symbols), of BzMA free-radical bulk homopolymerization at ambient pressure.

The Arrhenius expressions for $<k_{\mathrm{t}}>_{0<i<1000}$ and $k_{\mathrm{t}}^{0}$ of BzMA are presented by Eqs. 6.6 and 6.7, respectively:

$$
\begin{aligned}
& \ln \left[<k_{\mathrm{t}}>_{0<i<1000} /\left(\mathrm{L} \cdot \mathrm{mol}^{-1} \cdot \mathrm{s}^{-1}\right)\right]=17.53-656 \cdot\left(T^{-1} / \mathrm{K}^{-1}\right) \\
& \ln \left[k_{\mathrm{t}}^{0} /\left(\mathrm{L} \cdot \mathrm{mol}^{-1} \cdot \mathrm{s}^{-1}\right)\right]=18.82-661 \cdot\left(T^{-1} / \mathrm{K}^{-1}\right)
\end{aligned}
$$

(BzMA bulk homopolymerization at ambient pressure, $-20 \leq \Theta /{ }^{\circ} \mathrm{C} \leq 0$, monomer conversion range 0 to $20 \%$ ).

From the slopes of the straight lines in Fig. 6.16, the activation energies are estimated to be: $E_{\mathrm{A}}\left(<k_{\mathrm{t}}>_{0<i<1000}\right)=(5.5 \pm 3.1) \mathrm{kJ} \cdot \mathrm{mol}^{-1}$ and $E_{\mathrm{A}}\left(k_{\mathrm{t}}^{0}\right)=(5.5 \pm 2.8) \mathrm{kJ} \cdot \mathrm{mol}^{-1}$.

There is no literature data on the temperature dependence of $k_{\mathrm{t}}$ for BzMA so far. The majority of investigations presented in the literature are on styrene [32,33,34] several acrylates, like MA, BA, DA [7,8,27,35,36], as well as for MMA [7] and DMA [8,27]. The reported 
activation energies are rather low ranging between 3 and $8 \mathrm{~kJ} \cdot \mathrm{mol}^{-1}$ (see [7]). The obtained value for $E_{\mathrm{A}}\left(<k_{\mathrm{t}}>_{0<i<1000}\right)$ of BzMA is consistent with the diffusion (either segmental or translational) controlled nature of the termination reaction.

\subsection{CONCLUSIONS}

SP-PLP-ESR is a novel pulsed laser polymerization method for detailed measurements into termination rate coefficients, $k_{\mathrm{t}}$. In this method single pulse initiation is combined with timeresolved ESR detection of the decay in pulse-laser-induced radical concentration.

As was already mentioned, it is rather difficult to report tabulated values of $k_{\mathrm{t}}$ because of the chain-length dependence of the termination rate coefficient and because of its strong dependence on monomer conversion in some cases [37]. On the other hand, it is possible to give chain length-averaged $k_{\mathrm{t}}$ values for a specific monomer conversion, $\left\langle k_{\mathrm{t}}\right\rangle$, as well as chain length-averaged $k_{\mathrm{t}}$ values for a certain chain length region. In the present work all averaged $k_{\mathrm{t}}$ values are reported for the chain length region $1<i<1000$ and monomer conversion below 30 per cent.

The data for the systems studied in this work is summarized in Tab. 6.2.

\begin{tabular}{|c|c|c|c|c|c|c|c|}
\hline Monomer & $\begin{array}{c}k_{\mathrm{p}} / \\
\mathrm{L} \cdot \mathrm{mol}^{-1} \cdot \mathrm{s}^{-1}\end{array}$ & $\begin{array}{c}<k_{\mathrm{t}}>_{0<i<1000}{ }^{*} / \\
\mathrm{L} \cdot \mathrm{mol}^{-1} \cdot \mathrm{s}^{-1}\end{array}$ & $\begin{array}{c}k_{\mathrm{t}}^{0 \mathrm{I}} / \\
\mathrm{L} \cdot \mathrm{mol}^{-1} \cdot \mathrm{s}^{-1}\end{array}$ & $\begin{array}{c}k_{\mathrm{t}}^{0 \mathrm{II} /} \\
\mathrm{L} \cdot \mathrm{mol}^{-1} \cdot \mathrm{s}^{-1}\end{array}$ & $\alpha^{\mathrm{I}}$ & $\begin{array}{c}\alpha_{1} \\
i<100\end{array}$ & $\begin{array}{c}\alpha_{2} \\
100<i<1000\end{array}$ \\
\hline DMA & 256 & $1.3 \cdot 10^{6}$ & $3.8 \cdot 10^{6}$ & $8.0 \cdot 10^{6}$ & 0.34 & 0.50 & 0.18 \\
\hline CHMA & $250[38]$ & $2.2 \cdot 10^{6}$ & $7.6 \cdot 10^{6}$ & $1.9 \cdot 10^{7}$ & 0.32 & 0.49 & 0.22 \\
\hline BzMA & 274 [38] & $3.8 \cdot 10^{6}$ & $1.3 \cdot 10^{7}$ & $1.5 \cdot 10^{7}$ & 0.30 & 0.51 & 0.21 \\
\hline
\end{tabular}

All coefficients refer to $0{ }^{\circ} \mathrm{C}$ and ambient pressure

* obtained using equation for ideal kinetics (Eq. 3.6),

I obtained using Eq. 6.4

II obtained using Eq. 6.5 for intervals $i<100$

Tab. 6.2 Rate coefficients of bulk polymerization of some methacrylates at low conversion (0 to $30 \%)$.

The conversion dependence of the average termination rate coefficients, $\left\langle k_{\mathrm{t}}>_{1<i<1000}\right.$, in bulk homopolymerizations of DMA, CHMA and BzMA determined at $0{ }^{\circ} \mathrm{C}$ and ambient pressure are presented in Fig. 6.17.

Inspection of Fig. 6.17 shows that DMA, CHMA and BzMA exhibit a constant average $k_{\mathrm{t}}$ up to 30 per cent of conversion. 


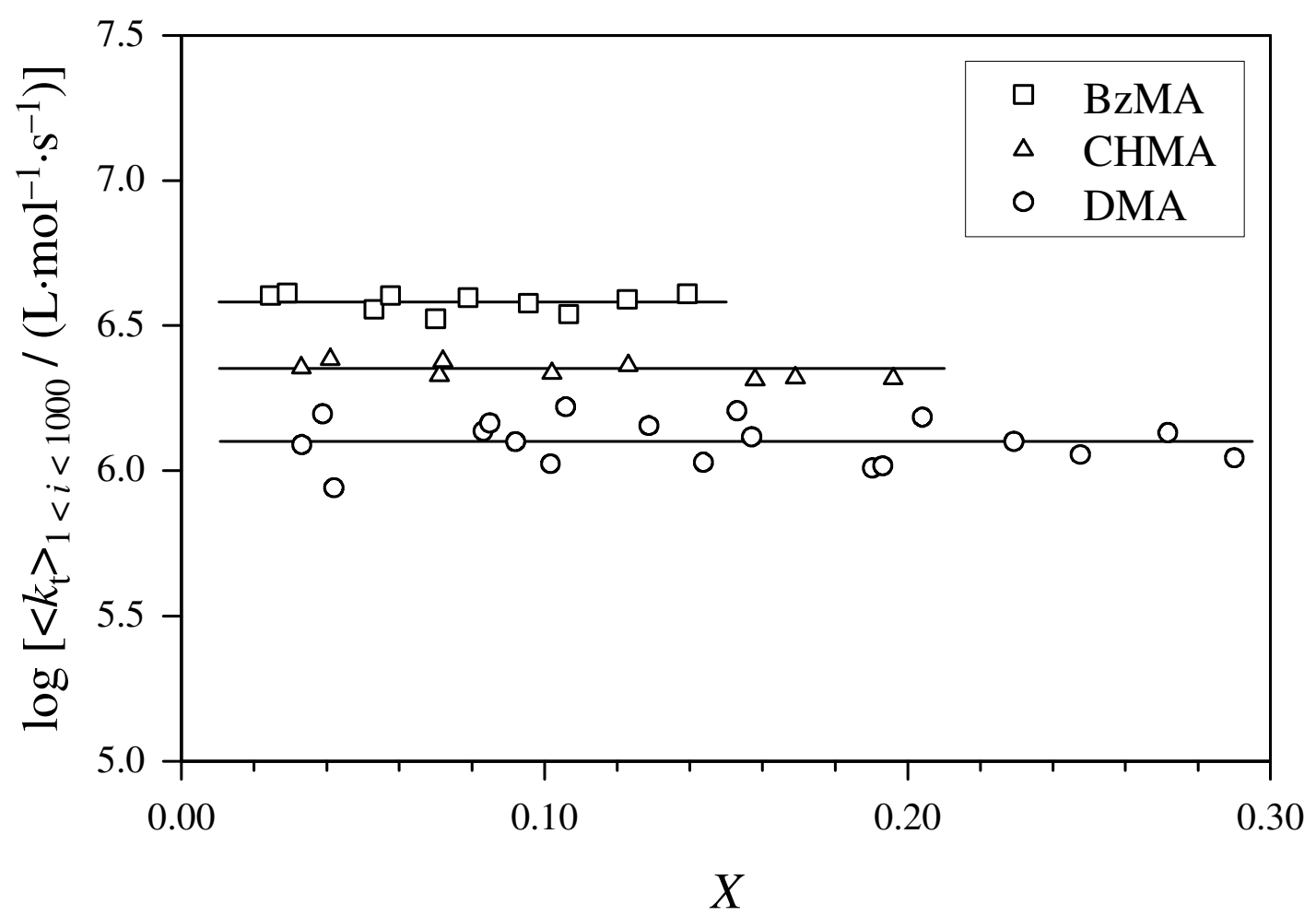

Fig. 6.17 The conversion dependence of the average termination rate coefficients, $<k_{\mathrm{t}}>_{1<i<1000}$, in bulk homopolymerizations of DMA (circles), CHMA (triangles) and BzMA (squares) determined at $0{ }^{\circ} \mathrm{C}$ and ambient pressure.

The results for DMA bulk polymerization show close agreement with the literature $k_{\mathrm{t}}$ data obtained using the established SP-PLP-NIR technique.

The termination rate coefficients between two monomeric free radicals, $k_{\mathrm{t}}^{0}$, are not measured directly in SP-PLP-ESR but result from the experimental data. Direct and reliable measurements of termination rate coefficients, $k_{\mathrm{t}}$, for a variety of small molecule radicals in a variety of solvents and over wide ranges of temperature have shown that in general one should have $k_{\mathrm{t}}^{0}=1 \cdot 10^{9} \mathrm{~L} \cdot \mathrm{mol}^{-1} \cdot \mathrm{s}^{-1}$ in free-radical polymerization $[39,40,41]$. In fact, it is easy to show that the Smoluchowski equation predicts $k_{\mathrm{t}}^{0} \approx 10^{9} \mathrm{~L} \cdot \mathrm{mol}^{-1} \cdot \mathrm{s}^{-1}$ for free-radical polymerization in dilute solution [42]. The values of $k_{\mathrm{t}}^{0}$ obtained in this work using Eq. 6.4 seem to be an underestimate. It could be explained as follows: the power-law dependence in Eq. 6.4 was derived for large coiled radicals [28]. Thus, $k_{\mathrm{t}}^{0}$ characterizes the termination behavior of a fictitious species which is large and coiled but of chain length unity. In other words, the obtained values of $k_{\mathrm{t}}^{0}$ are those that would be true if monomeric radicals behaved as macroradical coils. It comes as no surprise that $k_{\mathrm{t}}^{0}$, which is extrapolated from the data obtained for macroradicals, does not coincide with the experimentally determined radicalradical combination rate coefficients for small radicals. 
The results obtained so far provide strong support for the recently proposed composite model, in which termination rate coefficients, starting from small radicals, first considerably decrease, e.g., with a power-law exponent of $\alpha_{1}=0.5$, and subsequently, e.g., at chain lengths above $i=100$, decrease to a weaker extent, e.g., with the theoretically predicted power-law exponent $\alpha_{2}=0.16$.

SP-PLP-ESR is particularly well suited for studies into the termination kinetics of low $k_{\mathrm{p}}$ and low $k_{\mathrm{t}}$ monomers, such as methacrylates with bulky ester groups or itaconates. With ESR spectrometers of enhanced time resolution being available, SP-PLP-ESR should become widely applicable toward the detailed analysis of free-radical termination kinetics.

The given examples illustrate that the novel method allows the chain-length dependence of $k_{\mathrm{t}}$ to be investigated within the entire range of chain lengths. This is the main advantage of the SP-PLP-ESR technique in comparison with other SP-PLP methods, in which one is usually restricted to one particular chain length region. 


\subsection{REFERENCES}

[1] Buback, M.; Egorov, M.; Gilbert, R. G.; Kaminsky, O. F.; Olaj, G. T.; Russell, G. T.; Vana, P.; Zifferer, G. Macromol. Chem. Phys. 2002, 203, 2570.

[2] Olaj, O.F.; Schnöll-Bitai, I. Eur. Polym. J. 1989, 25, 635.

[3] Olaj, O.F.; Bitai, I.; Hinkelmann, F. Macromol. Chem. 1987, 188, 1689.

[4] Gao, J.; Penlidis, A. J. M. S. Rev. Macromol. Chem. Phys. 1996, C36, 199.

[5] Buback, M., Hippler, H., Schweer, J., Vögele, H.-P., Makromol. Chem., Rapid Commun. 1986, 7, 261.

[6] Beuermann, S.; Buback, M. Prog. Polym. Sci. 2002, 27, 191.

[7] Kowollik, C. Ph. D. Thesis, Göttingen, 1999.

[8] Feldermann, A. Ph. D. Thesis, Göttingen, 2003.

[9] Buback, M.; Egorov, M.; Junkers, T.; Panchenko, E. Macromol. Rapid Commun. 2004, 25, 1004.

[10] Smith, G. B.; Russell, G. T.; Heuts, J. P. A. Macromol. Theory Simul. 2003, 12, 299.

[11] Allen, P. E. M.; Patrick, C. R. Macromol. Chem. 1961, 47, 154.

[12] Olaj, O. F.; Kornherr, A.; Zifferer, G. Macromol. Rapid Commun. 1997, 18, 997.

[13] Olaj, O. F.; Kornherr, A.; Zifferer, G. Macromol. Rapid Commun. 1998, 19, 89.

[14] Olaj, O. F.; Vana, P.; Kornherr, A.; Zifferer, G. Macromo. Chem. Phys. 1999, 200, 2031.

[15] Schweer, J. Ph. D. Thesis, Göttingen, 1988.

[16] Le Guillou, J. C.; Zinn-Justin, J. Phys. Rev. B : Condens. Matter 1980, 21, 3976.

[17] Clay, P. A.; Gilbert, R. G.; Russell, G. T. Macromolecules 1987, 20, 850.

[18] Olaj, O. F.; Zifferer, G. Macromol. Chem., Rapid Commun. 1982, 3, 549.

[19] Khokhlov, A. R. Macromol. Chem., Rapid Commun. 1981, 2, 633.

[20] Piton, M. C.; Gilbert, R. G.; Chapmann, B. E.; Kuchel, P. W. Macromolecules 1993, $26,4472$.

[21] Kamachi, M. J. Polym. Sci. Part A: Polym. Chem. 2002, 40, 269.

[22] Kamachi, M.; Kajiwara, A. Macromol. Symp. 2002, 179, 53.

[23] Zhu, S.; Tian, Y.; Hamielec, A. E. Macromolecules 1990, 23, 1144.

[24] Junkers, T. Private communication.

[25] Buback, M.; Egorov, M.; Junkers, T.; Panchenko, E. Macromol. Chem. Phys. 2005, $206,333$.

[26] Vana, P.; Yee, L. H.; Davis, T.P. Macromolecules 2002, 35, 3008. 
[27] Buback, M.; Egorov, M.; Feldermann, A. Macromolecules 2004, 37, 1768.

[28] Fridman, B.; O’Shaughnessy,B. Macromolecules 1993, 26, 5726.

[29] Wisnudel, M.B.; Torkelson, J. M. J. Polym. Sci., Part B: Polym. Phys. 1996, 34, 2999.

[30] de Kock, J. B. L.; van Herk, A. M.; German, A. L. J. Macromol. Sci. Part C: Polym. Rev. 2001, 41, 199.

[31] Vana, P.; Davis, T. P.; Barner-Kowollik, C. Macromol. Rapid Commun. 2002, 23, 952.

[32] Buback, M.; Kuchta, F.-D. Macromol. Chem. Phys. 1997, 198, 1455.

[33] Ogo, Y.; Yokawa, M.; Imoto, T. Macromol. Chem. 1973, 171, 123.

[34] Moore, P. W.; Clouston, J. G.; Chaplin, R. P. J. Polymer Sci., Polym. Chem. Ed. 1981, 19, 1659 and 1671.

[35] Buback, M.; Kuelpmann, A.; Kurz, C. Macromol. Chem. Phys. 2002, 203, 1065.

[36] Kurz, C. Ph. D. Thesis, Göttingen, 1994.

[37] Handbook of Radical Polymerization, Matyjaszewski, K.; Davis, T. P., Eds.; WileyInterscience: New York, 2002.

[38] Beuermann, S.; Buback, M.; Davis, T. P.; Garcia, N.; Gilbert, R. G.; Hutchinson, R. A.; Kajiwara, A.; Kamachi, M.; Lacik, I.; Russell, G. T. Macromol. Chem. Phys. 2003, 204, 1338.

[39] Fischer, H.; Paul, H. Acc. Chem. Res. 1987, 20, 200.

[40] Mahabadi, H. K. Macromolecules 1985, 18, 1319.

[41] Buback, M.; Busch, M.; Kowollik, C. Macromol. Theory Simul. 2000, 9, 442.

[42] Russell, G. T. Macromol. Theory Simul. 1995, 4, 497. 


\section{Study into the OcCurrence of Secondary Propagating} AND Mid-Chain Radicals Via ESR in Free-Radical ACRYlate Polymerization

\subsection{INTRODUCTION}

Since 1987, when the PLP-SEC technique was introduced [1], the new method has been extensively used for the study of slowly propagating monomers, such as styrene [2] and methacrylates [3,4]. For high $k_{\mathrm{p}}$ monomers such as the acrylates the PLP-SEC procedure, which requires that laser pulsing is the dominant chain-starting and chain-stopping event, is less easily applied. A general problem with the acrylates is related to the fact that at temperatures above $30^{\circ} \mathrm{C}$, the PLP-SEC method results in broad molecular weight distributions which do not meet the requirements of a successful PLP-experiment [5]. It has been suggested that transfer process (transfer to monomer [5,6], transfer to polymer including intramolecular transfer (backbiting) $[7,8,9,10,11,12]$ ) deteriorate the MWD. For transfer to monomer it was soon realized that unrealistically high transfer rates were needed to explain the experiments [13]. Chain transfer to polymer on the other hand is a well known phenomenon and has received a lot of attention by the group of Lovell $[42,14,15]$. Under the low conversion conditions of PLP-SEC experiments, that is at low polymer contents, intramolecular chain transfer to polymer (backbiting) is particularly important.

The secondary propagating radicals (SPRs) resulting from propagation reactions are transferred into tertiary mid-chain radicals (MCR) via chain transfer to polymer processes (Fig. 7.1). In addition to propagation and termination, SPRs can undergo hydrogen-transfer reactions associated with shifting the radical functionality to a carbon atom on the same polymer chain (intramolecular transfer), preferably by the so-called backbiting process [16] which produces a mid-chain radical MCR1 (Fig. 7.1), or to the carbon atom of another polymer molecule (intermolecular transfer) which results in an MCR2 species (Fig. 7.1). Both types of transfer reactions primarily occur by hydrogen abstraction from carbon atoms which are activated by ester moiety. 


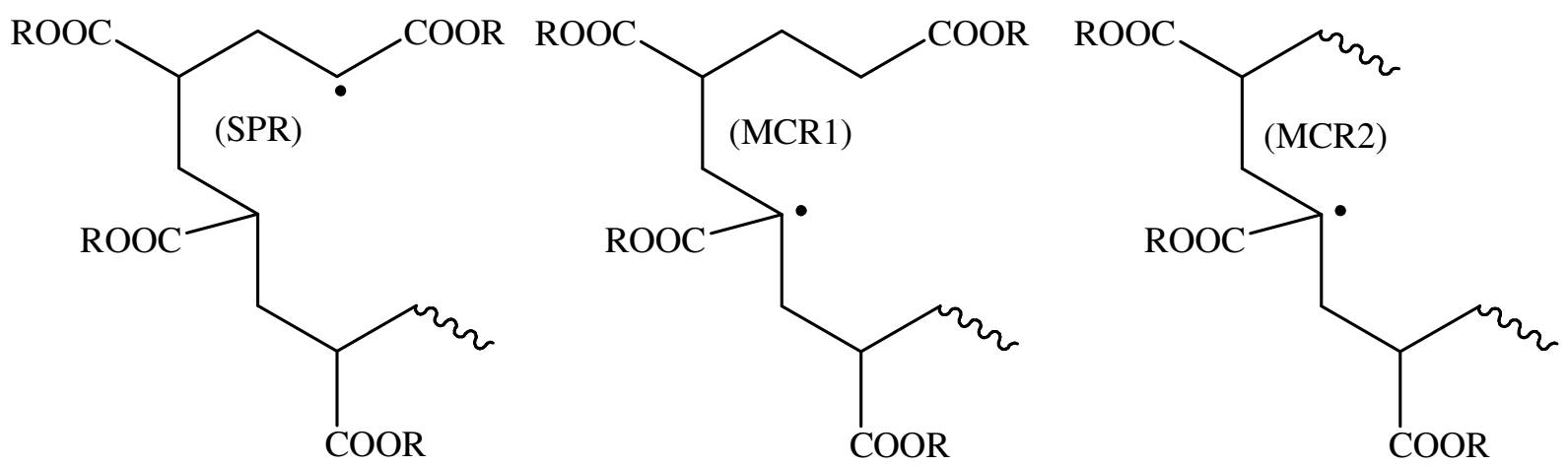

Fig. 7.1 Chemical structure of the secondary propagating radical (SPR) and of two types of tertiary radicals which are formed either by backbiting (MCR1) or by intramolecular transfer to more remote positions on the backbone and by intermolecular chain transfer reactions (MCR2).

The backbiting reactions are considered to occur as a $1,5-\mathrm{H}$ shift reaction via a six-membered transition structure [16], and hence a growing chain needs a minimum of three monomer units to undergo the backbiting reaction. The scheme of intramolecular chain transfer to polymer is given in Fig. 7.2.
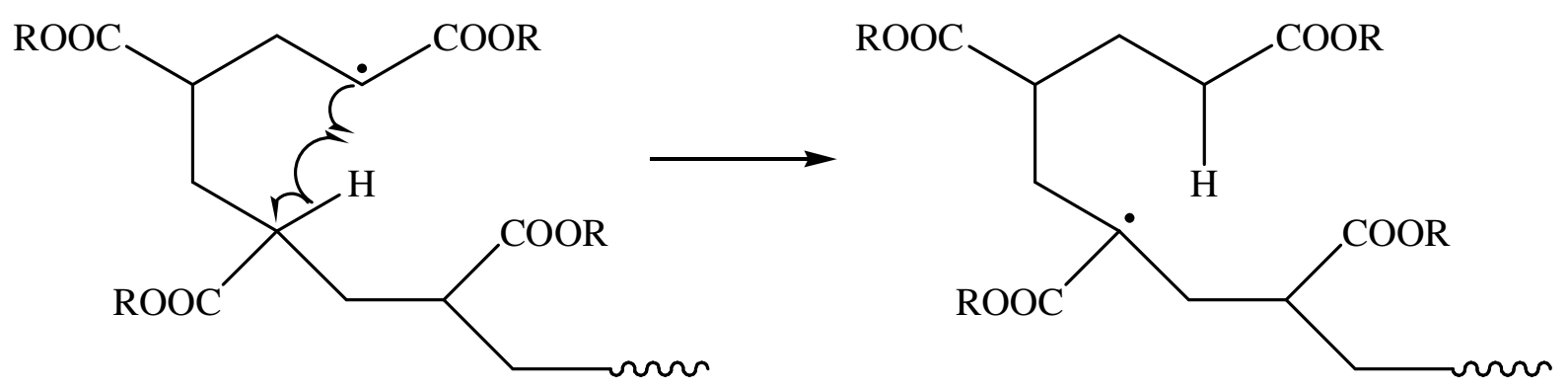

Fig. 7.2 The mechanism of intramolecular chain transfer (backbiting).

Because of the high propagation rate of acrylates [17], primary radical species from photoinitiator decomposition, small-size radicals from the initial propagation period and small radicals produced by chain transfer to monomer molecules are not easily seen in the ESR spectrum at typical polymerization conditions because of the very rapid addition of monomer molecules to these species. The radical species which are contributing to the ESR spectrum are shown in Fig. 7.1.

Intermolecular chain transfer may be neglected under PLP-SEC conditions, where monomer conversion and thus the amount of polymer are low. As backbiting is favored over 
intramolecular hydrogen transfer from arbitrary positions along the backbone of the macroradical other than the one which affords for $1,5-\mathrm{H}$ shift via the six-membered transition structure, MCR1 will be the preferred MCR species under PLP conditions. That SPR and MCR1 species dominate the PLP-ESR spectrum is for different reasons: (i) Rapid addition to acrylate monomer of SPRs does not diminish the concentration of this type of radicals as this process transforms one SPR species into another. (ii) The MCR1 radical is relatively stable with a significant steric hindrance contributing to the low addition rate to monomer of this species.

These tertiary radicals can either transform back into a secondary propagating radical via reinitiation, or undergo $\beta$-scission [43]. The latter reaction becomes important at temperatures above $80{ }^{\circ} \mathrm{C}$, and thus can be neglected at the temperatures at which experiments were carried out in this work. The re-initiation step on the other hand has been estimated via the kinetics of the sterically hindered butyl acrylate dimer [18] and methyl acrylate dimer [19]. Because of the low speed of this reaction, formation of MCR1 via backbiting is associated with macroradical chain length being no longer linearly related to the time $t$ after applying the laser pulse at $t=0$. Under polymerization conditions where only a small fraction of SPRs has not be converted into long-lived MCR1 (or MCR2) species in the time period between two successive laser pulses, the quality of $k_{\mathrm{p}}$ measurements via PLP-SEC may be intolerably reduced. Proof of the occurrence of mid-chain radicals has been obtained by electron spine resonance (ESR) spectroscopy under normal free-radical polymerization conditions [20], and by the observation of quaternary carbons with ${ }^{13} \mathrm{C}-\mathrm{NMR}$ [39] under pulsed laser polymerization conditions.

It is known from theory that the ESR technique can directly provide information on the structure, properties, and concentration of propagating radicals [21,22]. The interpretation of the ESR spectra of acrylate polymerizations turned out to be rather complicated. There have been some unsolved problems with the ESR spectra of propagating radicals of acrylates. Several kinds of ESR spectra under various conditions were reported to be the ones of secondary propagating radicals [23,24,25,26,27,28]. Also for mid-chain radicals no final understanding of the ESR spectra has been reached so far [26,27,28,29]. A major reason behind the discrepancies is seen in polymerization conditions and polymer properties being dissimilar. The differences affect the dynamics of molecular reorientation processes and thus can modify hyperfine splitting.

In the present work the ESR spectra of propagating radicals of acrylates at several temperatures have been studied. The percentage of mid-chain radicals is determined by 
assuming that the measured ESR spectrum is composed of a linear combination of the spectrum for terminal secondary radicals and the spectrum for tertiary mid-chain radicals. A least squares method was used to fit the simulated ESR spectra of these two species to the experimental spectra (after noise reduction by Fourier transformation). Within the fitting procedure, a triangular weighting function with maximum sensitivity at the central position of the ESR spectrum has been used. The experimental spectra shown in the following sections are the measured ones without any data treatment.

\subsection{ESR Measurements of Propagating Radicals of ACrylates}

\subsubsection{ESR Measurements during BA Polymerization}

There are contradicting interpretations in literature with respect to the interpretation of the ESR spectra of acrylates. Two papers confirming the occurrence of mid-chain radicals for cyclohexyl acrylate [28] and phenyl acrylate [27] by ESR measurements were published by Yamada and co-workers. In their work, the interpretation of ESR spectra, obtained at $60{ }^{\circ} \mathrm{C}$ in benzene and AIBN as initiator, were assigned to a combination of SPRs with a 7-line spectrum and MCRs with a 3-line spectrum with a coupling constant of $\alpha=27.5 \mathrm{G}$. With respect to this latter spectrum, Yamada et al. [27,28] assumed that only two out of the four hydrogens can couple with the unpaired electron of the mid-chain radical. In order to support their findings, these authors referred to earlier work carried out on acrylate systems $[24,25,26,30]$. Interestingly, the early work on the occurrence of mid-chain radicals was carried out on cross-linked systems where the low mobility of the polymer chains may be responsible for the coupling of only two out of the four $\beta$-hydrogen atoms with the unpaired electron. Kim et al., however, demonstrated for mid-chain radicals in a butyl acrylate film, that increasing temperature enhances the number of lines in the MCR ESR spectrum. The authors attributed this effect to the coupling of the radical functionality with two sets of slightly non-equivalent protons [31].

Kajiwara and Kamachi [20] reported on changes of the ESR spectra in the solution polymerization of tert-butyl acrylate at temperatures between $-30{ }^{\circ} \mathrm{C}$ and $60{ }^{\circ} \mathrm{C}$. In the initial stage of the polymerization at $-30{ }^{\circ} \mathrm{C}$, a 6 -line spectrum with narrow line width was observed. This spectrum is assigned to the SPR with the $\alpha$-proton $\left(\alpha_{\mathrm{H} \alpha}=2.28 \mathrm{mT}\right)$ and with two $\beta$ methylene protons $\left(\alpha_{\mathrm{H} \beta}=2.06 \mathrm{mT}\right)$. The spectrum had a very short lifetime and readily changed to a totally different, long living 7-line spectrum obtained at $60{ }^{\circ} \mathrm{C}$ with broader line 
width, which looks very similar to the one reported by Azukizawa et al. [27]. The interpretation of the ESR spectrum of the propagating SPRs provided by Kajiwara and Kamachi thus is slightly different from the one given by Azukizawa et al. [27]. Kajiwara and Kamachi [20] leave a question mark on the interpretation of the spectra at elevated temperatures.

In the present study, ESR experiments were carried out for $n$-butyl acrylate in toluene, under PLP conditions (frequency of $20 \mathrm{~Hz}$ ), in an effort to determine mid-chain radical concentrations as a function of temperature. PLP-ESR experiments were carried out in the temperature range of $-50{ }^{\circ} \mathrm{C}$ up to $70{ }^{\circ} \mathrm{C}$ at ambient pressure. All ESR spectra were recorded at low degree of monomer conversions. The total recording time of one single scan was about $5 \mathrm{~s}$.

(a)

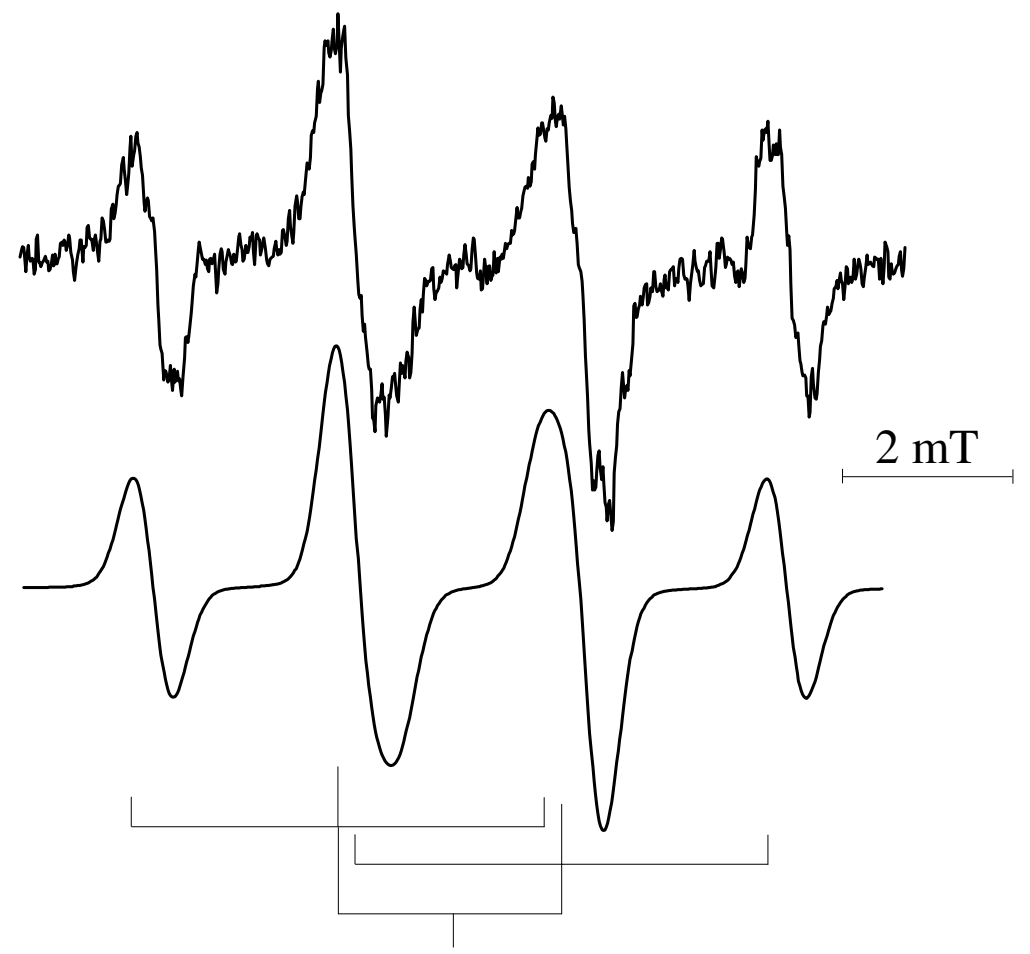

Fig. 7.3 ESR spectrum for $n$-butyl acrylate polymerization, (a) experimental curve recorded during the initial period of a quasi-stationary solution (in toluene) polymerization at $-50{ }^{\circ} \mathrm{C}$ initiated with $351 \mathrm{~nm}$ excimer laser pulses applied at a repetition rate of $20 \mathrm{~Hz} ; c_{\mathrm{BA}}=1.52 \mathrm{~mol} \cdot \mathrm{L}^{-1}, c_{\mathrm{MMMP}}=9.0 \cdot 10^{-3} \mathrm{~mol} \cdot \mathrm{L}^{-1}$; (b) simulated spectrum using hyperfine coupling constants of $\alpha_{\mathrm{H \alpha}}=2.35 \mathrm{mT}$ and $\alpha_{\mathrm{H} \beta}=2.04 \mathrm{mT}$.

Shown in Fig. 7.3 is the ESR spectrum measured during the initial period of a pulsed laser $n$ butyl acrylate polymerization in toluene solution at $-50{ }^{\circ} \mathrm{C}$. A spectrum with four broad lines 
is seen which is very similar to the one reported by Kajiwara and Kamachi for tert-butyl acrylate polymerization at $-30{ }^{\circ} \mathrm{C}$. The four-line spectrum actually consists of six components with two pairs of components being overlapping, as is indicated by the assignment bars in the lower part of Fig. 7.3. The spectrum is due to the SPR species in which the radical functionality couples with the $\alpha$-proton and with two equivalent $\beta$-methylene protons. The hyperfine coupling constants, $\alpha_{\mathrm{H} \alpha}=2.35 \mathrm{mT}$ and $\alpha_{\mathrm{H} \beta}=2.04 \mathrm{mT}$, are in close agreement with data reported by Kajiwara and Kamachi for tert-butyl acrylate [20,34].

The ESR spectrum obtained in the initial period of $n$-butyl acrylate solution PLP at $60{ }^{\circ} \mathrm{C}$, shown in Fig. 7.4, is significantly different from the ESR spectrum recorded at $-50{ }^{\circ} \mathrm{C}$ (Fig. 7.3).

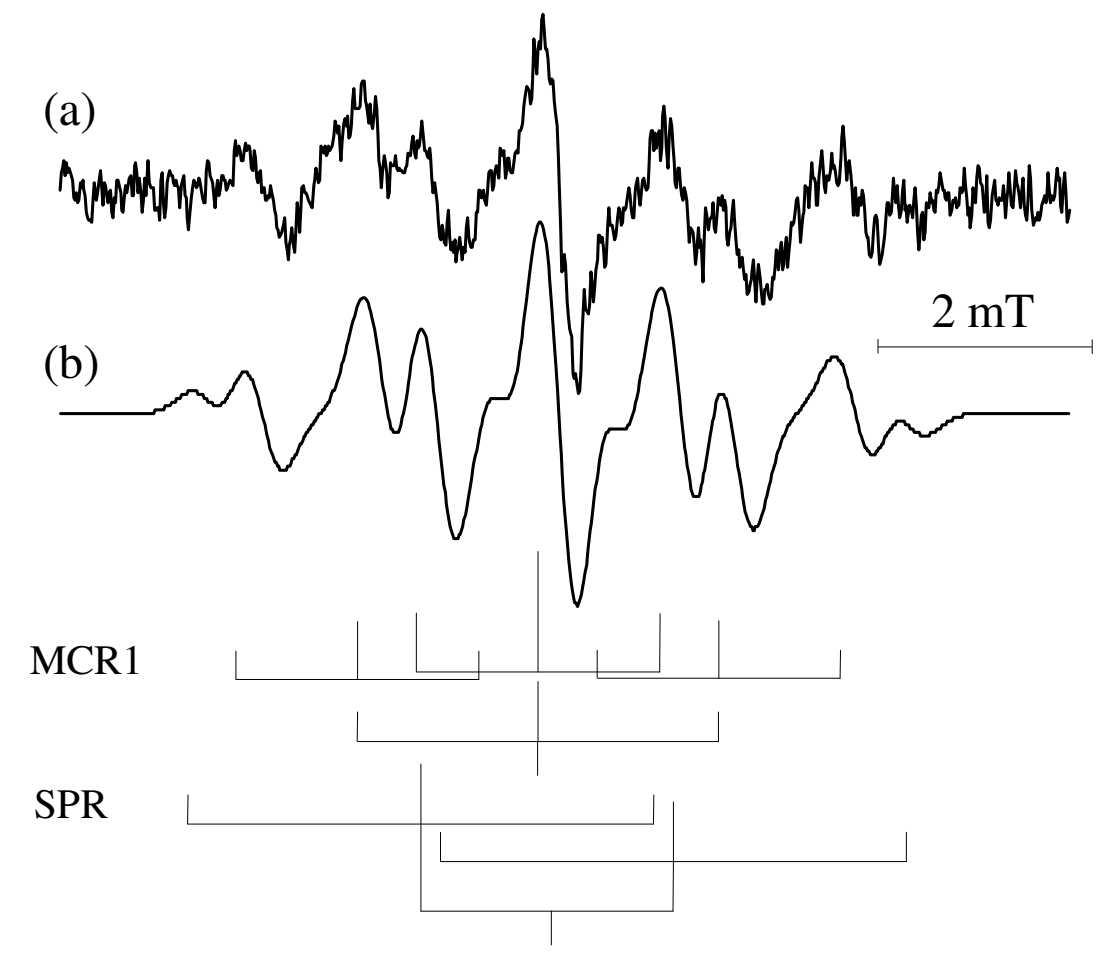

Fig. 7.4 ESR spectrum for $n$-butyl acrylate polymerization, (a) experimental curve recorded during the initial period of a quasi-stationary solution (in toluene) polymerization at $60{ }^{\circ} \mathrm{C}$ initiated with $351 \mathrm{~nm}$ excimer laser pulses applied at a repetition rate of $20 \mathrm{~Hz}, c_{\mathrm{BA}}=1.52 \mathrm{~mol} \cdot \mathrm{L}^{-1}, c_{\mathrm{MMMP}}=9.0 \cdot 10^{-3} \mathrm{~mol} \cdot \mathrm{L}^{-1}$; (b) simulated spectrum using hyperfine coupling constants of $\alpha_{\mathrm{H} \beta 1}=1.09 \mathrm{mT}$ and $\alpha_{\mathrm{H} \beta 2}=1.64 \mathrm{mT}$ for fitting the 9-line spectrum of MCR1 and using $\alpha_{\mathrm{H} \alpha}=2.35 \mathrm{mT}$ and $\alpha_{\mathrm{H} \beta}=2.04 \mathrm{mT}$ (as in Fig. 7.3) for fitting the SPR signal. The contribution of the MCR1 spectrum to the entire ESR signal corresponds to a fraction of 84 per cent MCR1 species being present. 
Visual inspection tells that the spectrum consists of more than ten components. Linear combination of the 4-line spectrum for the SPR species with a 3-line spectrum (as used for representing the ESR contour of the MCR so far [27,28,32]), thus will not afford for an adequate fit of the entire ESR spectrum. On the other hand, no reason is seen why, for $n$-butyl acrylate polymerization at $60{ }^{\circ} \mathrm{C}$ with pulsed laser initiation being used for controlling polymer size, the dynamics of MCRs should not be sufficiently large to provide identical coupling situations of the radical functionality with each of the two protons of the two adjacent methylene moieties. In MCR1, which is the by far dominant mid-chain radical species under PLP-SEC polymerization conditions, these two $\mathrm{CH}_{2}$ moieties are slightly different (see Fig. 7.1), as MCR1 has a short-armed and a long-armed branch. As a consequence, it appears reasonable, if not mandatory, to consider a 9-line spectrum for representation of MCR1. The simulated ESR contour in Fig. 7.4 which results from a linear combination of a 9-line spectrum for MCR1 with an SPR spectrum (with the coupling constants as used for the fit in Fig. 7.3) indeed shows nice agreement with the measured ESR spectrum. The hyperfine coupling constants for the two types of slightly dissimilar methylene protons are: $\alpha_{\mathrm{H} \beta 1}=1.09 \mathrm{mT}$ and $\alpha_{\mathrm{H} \beta 2}=1.64 \mathrm{mT}$.

During BA polymerization initiated by thermal decomposition of TBPO (Fig. 7.5) spectra similar to the already observed ones for the laser initiated polymerization of BA were obtained.

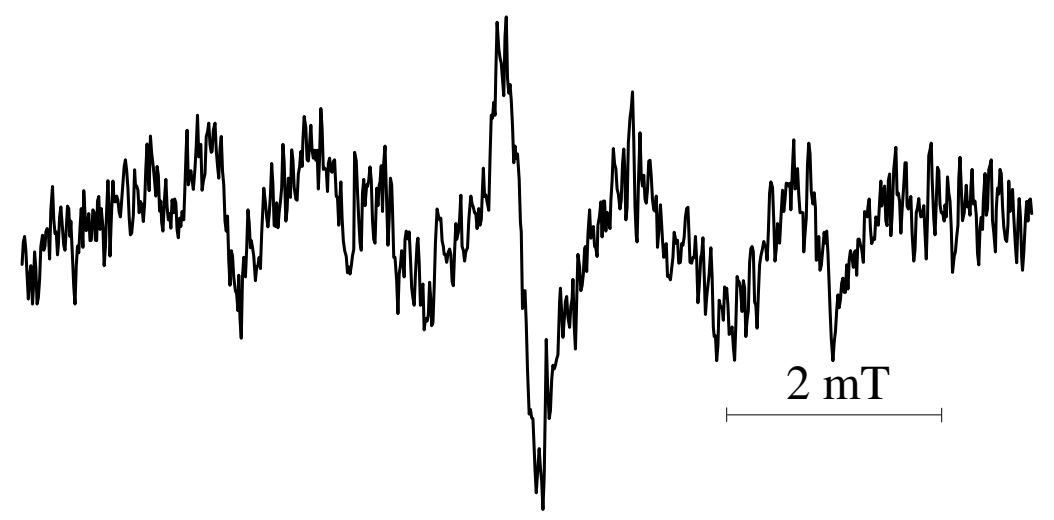

Fig. 7.5 ESR spectrum observed during BA polymerization initiated by the thermal decomposition of TBPO in toluene at $65^{\circ} \mathrm{C}, c_{\mathrm{BA}}=1.74 \mathrm{~mol} \cdot \mathrm{L}^{-1}$, $c_{\mathrm{TBPO}}=5.8 \cdot 10^{-2} \mathrm{~mol} \cdot \mathrm{L}^{-1}$. 
As is demonstrated by Fig. 7.6 and Fig. 7.7, also at intermediate polymerization temperatures of $-20^{\circ} \mathrm{C}$ and $30^{\circ} \mathrm{C}$, respectively, the overall contour of the ESR spectrum is adequately fitted by assuming an overlap of a 4-line SPR spectrum with a 9-line spectrum for MCR1.

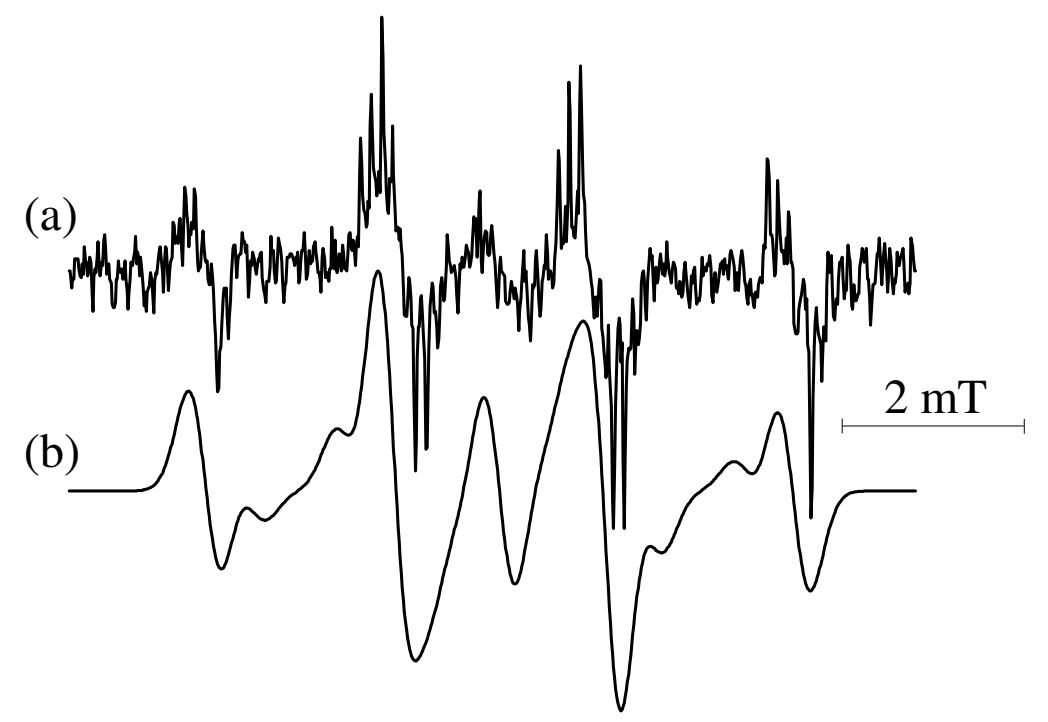

Fig. 7.6 ESR spectrum for $n$-butyl acrylate polymerization, (a) experimental curve recorded during the initial period of a quasi-stationary solution (in toluene) polymerization at $-20{ }^{\circ} \mathrm{C}$ initiated with $351 \mathrm{~nm}$ excimer laser pulses applied at a repetition rate of $20 \mathrm{~Hz} ; c_{\mathrm{BA}}=1.52 \mathrm{~mol} \cdot \mathrm{L}^{-1}, c_{\mathrm{MMMP}}=9.0 \cdot 10^{-3} \mathrm{~mol} \cdot \mathrm{L}^{-1}$; (b) simulated spectrum using the hyperfine coupling constants as in Fig. 7.4. The contribution of the MCR1 spectrum to the entire ESR signal corresponds to a fraction of 36 per cent MCR1 species. 


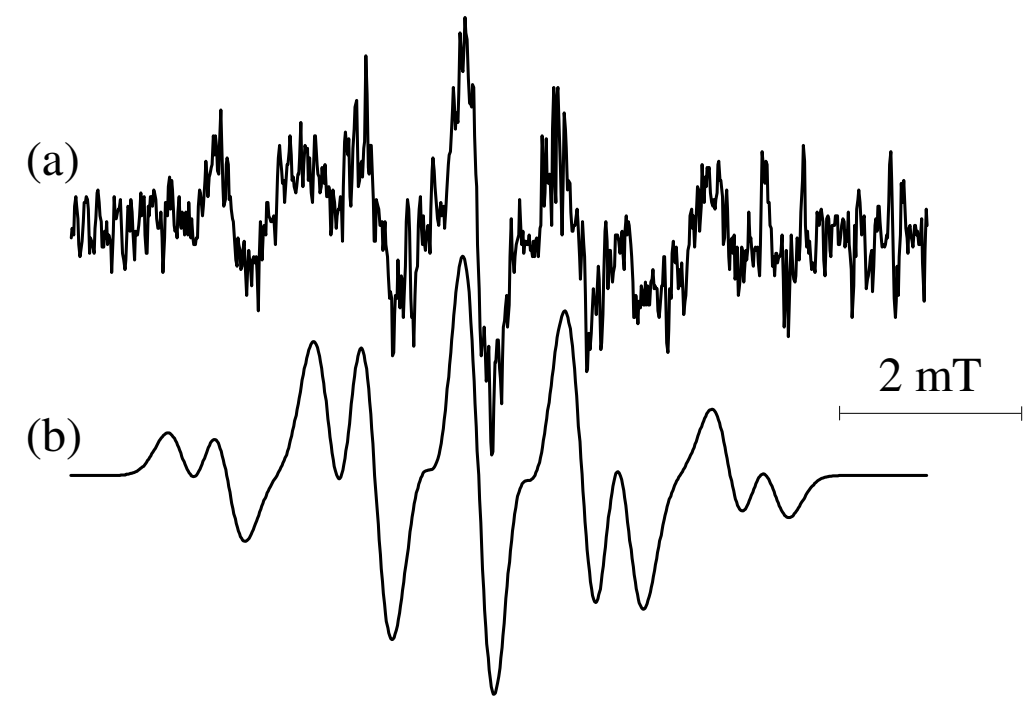

Fig. 7.7 ESR spectrum for $n$-butyl acrylate polymerization, (a) experimental curve recorded during the initial period of a quasi-stationary solution (in toluene) polymerization at $30^{\circ} \mathrm{C}$ initiated with $351 \mathrm{~nm}$ excimer laser pulses applied at a repetition rate of $20 \mathrm{~Hz} ; c_{\mathrm{BA}}=1.52 \mathrm{~mol} \cdot \mathrm{L}^{-1}, c_{\mathrm{MMMP}}=9.0 \cdot 10^{-3} \mathrm{~mol} \cdot \mathrm{L}^{-1} ;(\mathrm{b})$ simulated spectrum using the hyperfine coupling constants as in Fig. 7.4. The contribution of the MCR1 spectrum to the entire ESR signal corresponds to a fraction of 76 per cent MCR1 species.

The spectra in Fig. 7.6 and Fig. 7.7 are of poorer signal-to-noise quality than are the ESR spectra taken at both lower and higher temperature. That lower $T$ improves the quality of ESR spectra is well known. The improvement toward higher temperature, in the particular situation of acrylate free-radical polymerization, is due to the fact that at higher $T$ the majority of the radicals are long-lived MCR1 species.

Whereas the low-temperature ESR spectra of this work are close to the literature ones, the ESR spectra measured during polymerization at high temperature are less similar. The reason behind this discrepancy is seen in differences of polymerization conditions other than temperature. Thus the studies by Yamada and colleagues $[27,28,33]$ and by Kajiwara and Kamachi [20,34] have been carried out at higher degrees of monomer conversion, which may lead to the occurrence of both MCR1 and MCR2 species. High conversion may also affect the reorientation dynamics of macroradicals which in turn may be reflected in the shape of the ESR spectrum as, e.g., two methylene protons being equivalent or non-equivalent influences hyperfine splitting. In addition to coil dynamics, also the kinetics of interconversion of different types of radicals may be largely affected by the polymerization conditions. 
Sato et al. [33] pointed out that the rate of interconversion between terminal and mid-chain radicals may occur with a complex kinetic scheme that includes backbiting and intermolecular hydrogen transfer with both processes being followed by addition to monomer and/or fragmentation by $\beta$-scission. These PLP-ESR studies into $n$-butyl acrylate polymerization appear to deal with a simplified system, one in which, at low degrees of monomer conversion, intermolecular hydrogen transfer plays no major role and where temperature is not sufficiently high to induce a large extent of $\beta$-scission subsequent to backbiting.

Presented in Fig. 7.8 are the results of the deconvolution of the measured ESR spectra into a 4-line component assigned to SPR and into a 9-line component assigned to MCR1. The contribution of these individual components to the overall ESR shape yields the fraction of the two types of radicals. Plotted in Fig. 7.8 is $x$, the percentage of mid-chain radicals MCR1, as a function of polymerization temperature at three initial $n$-butyl acrylate concentrations, $c_{\mathrm{BA}}$. 


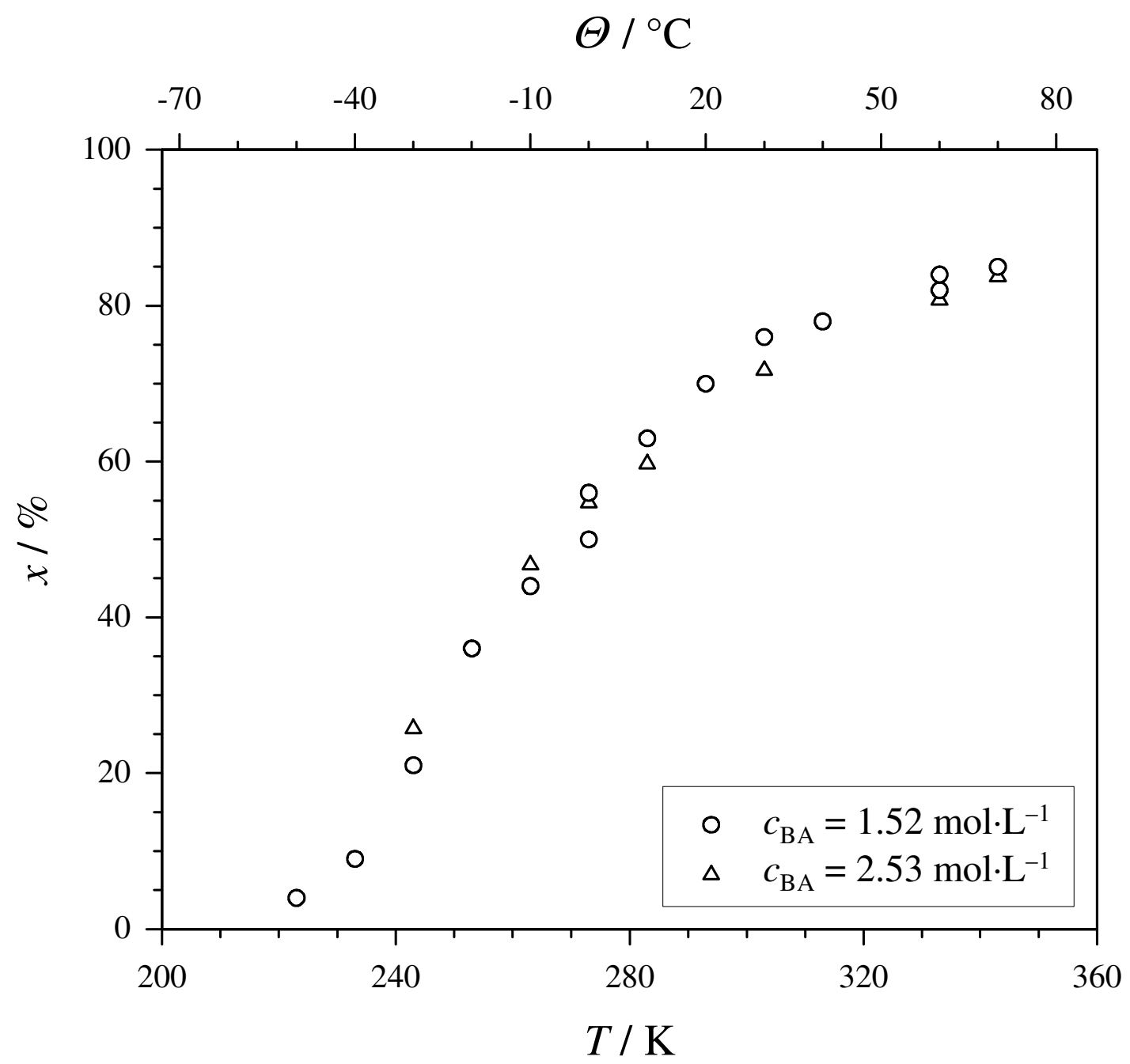

Fig. 7.8 Percentage of mid-chain radicals, $x$, plotted as a function of polymerization temperature, $T$, for quasi-stationary $n$-butyl acrylate solution polymerizations induced by $351 \mathrm{~nm}$ excimer laser pulses applied at a repetition rate of $20 \mathrm{~Hz}$. The data refers to the low-conversion regime of polymerizations with two different initial $n$-butyl acrylate concentrations, $c_{\mathrm{BA}}, c_{\mathrm{MMMP}}=9.0 \cdot 10^{-3} \mathrm{~mol} \cdot \mathrm{L}^{-1}$.

At the lowest temperature, the amount of mid-chain radicals is almost negligible and polymerization essentially occurs via SPR species. With increasing temperature, the fraction of MCRs largely increases and becomes as large as 75 per cent at $30^{\circ} \mathrm{C}$. This observation explains the enormous difficulties seen with PLP-SEC experiments on acrylates at temperatures above $30^{\circ} \mathrm{C}$. The large fraction of mid-chain radicals scrambles the narrow size distribution of propagating radicals and gives rise to broad structureless SEC curves for the PLP-produced polymeric products. This finding is in full agreement with Monte Carlo simulations by Arzamendi et al. [7]. It should be noted that a remarkably high fraction of midchain radicals has also been observed by the Yamada group [33] within the ESR studies into 
UV-induced EHA polymerization. These authors report a mid-chain radical concentration of 70 per cent for $25^{\circ} \mathrm{C}$ which value is in close agreement with the one observed for PLP polymerization of $n$-butyl acrylate (Fig. 7.8).

The data in Fig. 7.8 for $n$-butyl acrylate concentrations between 1.52 and $2.53 \mathrm{~mol} \cdot \mathrm{L}^{-1}$ suggest no clear effect of monomer concentration on the percentage of mid-chain radicals. This result may be understood as being due to the enormous difference in monomer and radical concentrations. Thus each radical is surrounded by a sufficiently large number of monomer molecules and the radical-monomer reaction will not be significantly affected by varying $n$-butyl acrylate content. Assuming that the stationary fraction of mid-chain radicals under PLP-SEC conditions is given by the difference in the rates of backbiting of an SPR species, which produces mid-chain radicals MCR1, and of monomer-addition reaction within the resulting monomer-complexed MCR1 species, referred to as MCR1*, yields the following expression:

$$
c_{\mathrm{SPR}} / c_{\mathrm{MCR} 1} *=k_{\mathrm{p}} * / k_{\mathrm{bb}}
$$

where $k_{\mathrm{bb}}$ is the backbiting rate coefficient of SPRs and $k_{\mathrm{p}} *$ is the propagation rate coefficient of MCR1* in the monomer-complexed MCR1* species. Eq. 7.1 may be re-arranged into an expression in terms of $x$, the percentage of MCR 1 species.

$$
x /(1-x)=k_{\mathrm{bb}} / k_{\mathrm{p}} *
$$




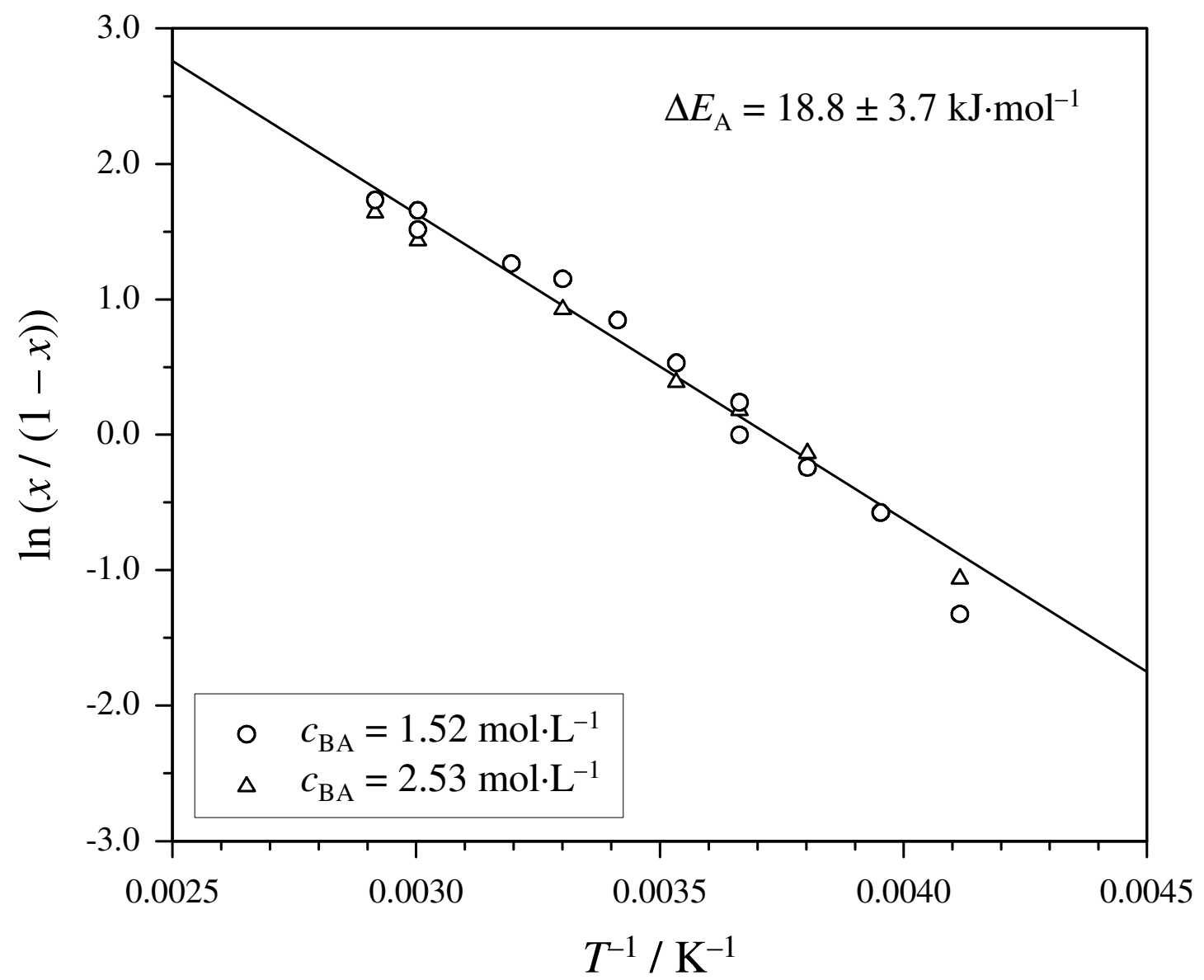

Fig. 7.9 Plot of $\ln (x /(1-x))$ vs. $T^{-1}$ for pulsed laser polymerizations of $n$-butyl acrylate (see text).

In Fig. 7.9, the term on the r.h.s. of Eq. 7.2 is plotted vs. $T^{-1}$ for $n$-butyl acrylate. According to the r.h.s. of Eq. 7.2, the linear slope of the Arrhenius-type plot of $k_{\mathrm{bb}} / k_{\mathrm{p}} *$ should yield the difference between the activation energies of backbiting of an SPR species, $E_{\mathrm{A}}\left(k_{\mathrm{bb}}\right)$, and of the propagation reaction from an MCR1* species, $E_{\mathrm{A}}\left(k_{\mathrm{p}} *\right)$. The data in Fig. 7.9, which are the ones for conditions where the concentration ratio of MCR1 to SPR radicals is in the range 0.1 and 10, closely fit to a straight line. The difference of activation energies turns out to be: $\Delta E_{\mathrm{A}}=E_{\mathrm{A}}\left(k_{\mathrm{bb}}\right)-E_{\mathrm{A}}\left(k_{\mathrm{p}} *\right)=18.8 \pm 3.7 \mathrm{~kJ} \cdot \mathrm{mol}^{-1}$. With independent information becoming available for $k_{\mathrm{p}}{ }^{*}$, absolute numbers for $k_{\mathrm{bb}}$ may be estimated from the ESR-spectroscopically measured percentages of mid-chain radicals. The simplifying assumptions underlying Eqs. 7.1 and 7.2 have to be kept in mind. It seems, however, that they are valid under the PLP-SEC conditions used in the present study. It appears to be a matter of priority to extend such studies to pulsed laser induced polymerization at higher conversion, where MCR2 species significantly come into play. The difficulties in interpreting ESR spectra at such higher degrees of monomer conversion, which are due to having a mixture of different types of mid- 
chain radicals and to effects of chain dynamics on hyperfine coupling constants, may perhaps be overcome by such experiments with pulsed laser initiation.

The large fraction of MCR1 mid-chain radicals which is associated with a small number of SPR species that do not undergo backbiting in the time period between two successive laser pulses adequately explains the difficulties of applying the PLP-SEC methodology toward $k_{\mathrm{p}}$ analysis of acrylates at temperatures of $30^{\circ} \mathrm{C}$ and above.

\subsubsection{ESR MEASUREMENTS DURING DA POLYMERIZATION}

For $n$-dodecyl acrylate, ESR experiments were carried out in toluene under PLP conditions (frequency of $20 \mathrm{~Hz}$ ), in an effort to determine the amount of mid-chain radicals as a function of temperature. ESR-PLP experiments were carried out at ambient pressure in the temperature range of $-20^{\circ} \mathrm{C}$ up to $60{ }^{\circ} \mathrm{C}$. The spectrum shown in Fig. 7.10 could be attributed to a linear combination of the 4-line spectrum for the SPR (36\%) with one $\alpha$-proton and two $\beta$-methylene protons with coupling constants $\alpha_{\mathrm{H} \alpha}=2.38 \mathrm{mT}$ and $\alpha_{\mathrm{H} \beta}=2.08 \mathrm{mT}$ and a 9-line spectrum for the MCR1 (64\%) having two sets of slightly unequal protons with coupling constants $\alpha_{\mathrm{H} \beta 1}=1.09 \mathrm{mT}$ and $\alpha_{\mathrm{H} \beta 2}=1.69 \mathrm{mT}$. 


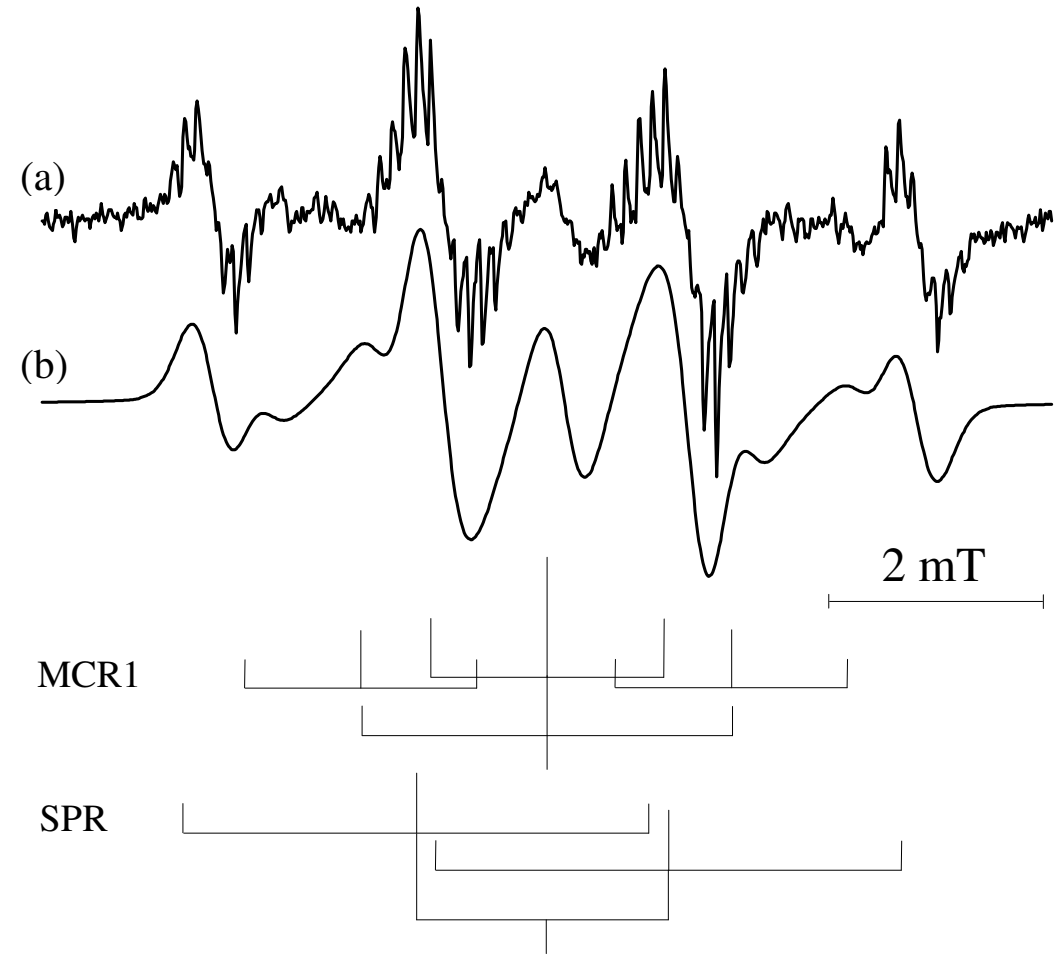

Fig. 7.10 (a) ESR spectrum observed during quasi-stationary DA polymerization initiated by laser pulses applied at a repetition rate of $20 \mathrm{~Hz}$ in toluene at $-20{ }^{\circ} \mathrm{C}, c_{\mathrm{DA}}=0.92 \mathrm{~mol} \cdot \mathrm{L}^{-1}, c_{\mathrm{MMMP}}=6.5 \cdot 10^{-3} \mathrm{~mol} \cdot \mathrm{L}^{-1}$ and $(\mathrm{b})$ its simulation with coupling constants $\alpha_{\mathrm{H} \alpha}=2.38 \mathrm{mT}$ and $\alpha_{\mathrm{H} \beta}=2.08 \mathrm{mT}$ for the SPR and $\alpha_{\mathrm{H} \beta 1}=1.09 \mathrm{mT}, \alpha_{\mathrm{H} \beta 2}=1.69 \mathrm{mT}$ for the MCR1.

In case of the DA laser initiated polymerization at $60{ }^{\circ} \mathrm{C}$ the ESR spectrum shown in Fig. 7.11 could be almost completely ascribed to a 9-line spectrum of the MCRs (97\%). 


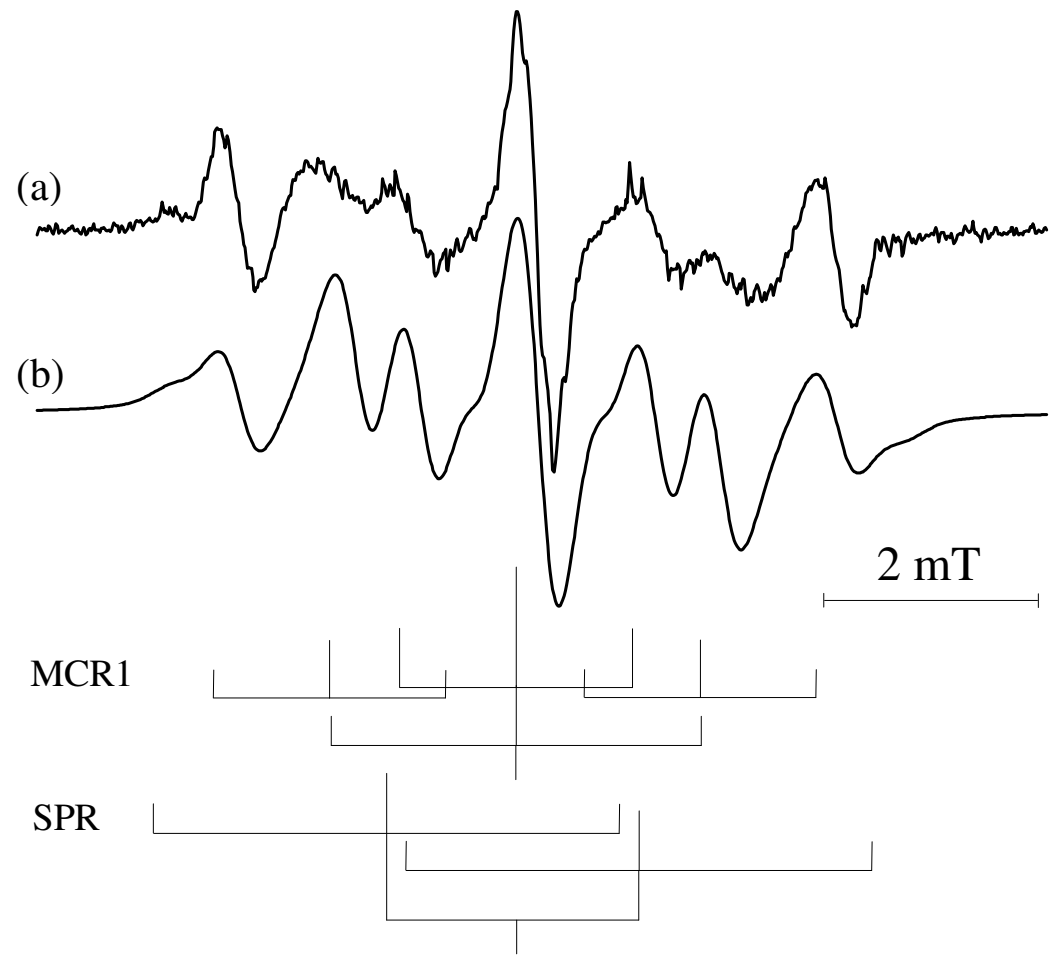

Fig. 7.11 (a) ESR spectrum observed during quasi-stationary DA polymerization initiated by laser pulses applied at a repetition rate of $20 \mathrm{~Hz}$ in toluene at $60{ }^{\circ} \mathrm{C}$, $c_{\text {DA }}=0.92 \mathrm{~mol} \cdot \mathrm{L}^{-1}, c_{\text {MMMP }}=6.5 \cdot 10^{-3} \mathrm{~mol} \cdot \mathrm{L}^{-1}$ and (b) its simulation with coupling constants $\alpha_{\mathrm{H} \alpha}=2.38 \mathrm{mT}$ and $\alpha_{\mathrm{H} \beta}=2.08 \mathrm{mT}$ for the SPR and $\alpha_{\mathrm{H} \beta 1}=1.09 \mathrm{mT}, \alpha_{\mathrm{H} \beta 2}=1.69 \mathrm{mT}$ for the MCR1.

During DA polymerization initiated by thermal decomposition of TBPO at $60{ }^{\circ} \mathrm{C}$ ESR spectra were obtained (see Fig. 7.12) that are very similar to the spectra observed in laser initiated polymerization.

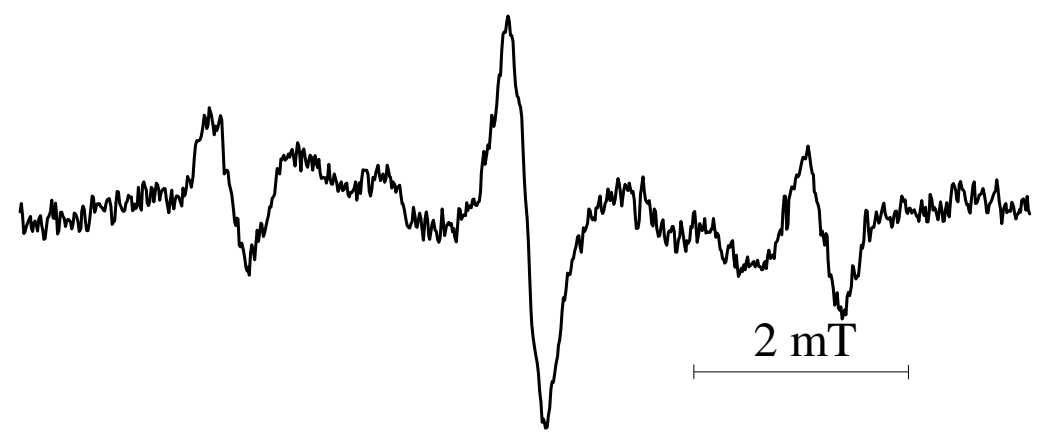

Fig. 7.12 ESR spectrum observed during DA polymerization initiated by the thermal decomposition of TBPO in toluene at $60{ }^{\circ} \mathrm{C}, c_{\mathrm{DA}}=0.93 \mathrm{~mol} \cdot \mathrm{L}^{-1}$, $c_{\mathrm{TBPO}}=12 \cdot 10^{-2} \mathrm{~mol} \cdot \mathrm{L}^{-1}$. 
For the determination of the amount of mid-chain radicals in free-radical polymerization of DA the same strategy was used as it was described in section 7.2.1. The obtained results are presented in Fig. 7.13.

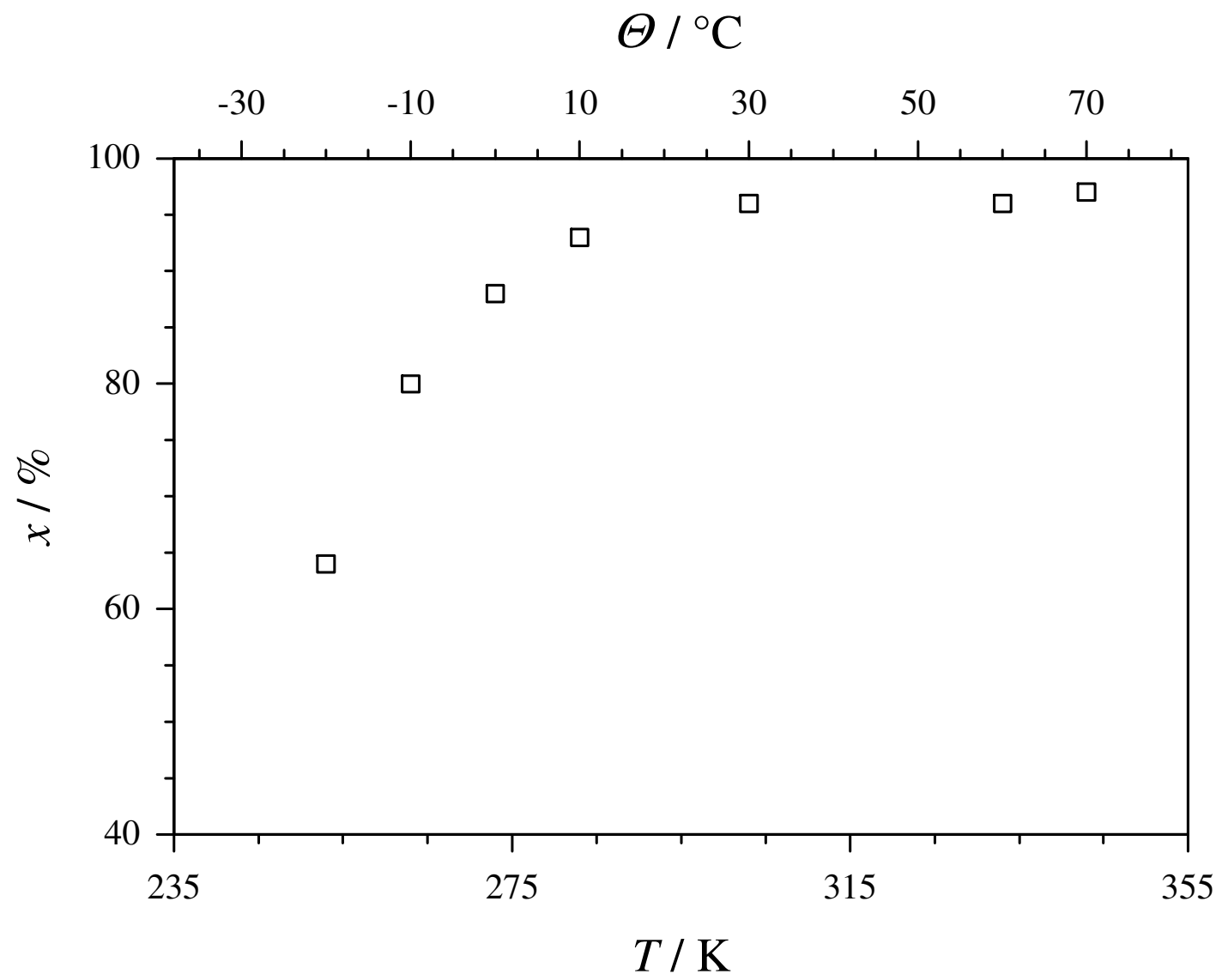

Fig. 7.13 Percentage of mid-chain radical, $x$, plotted as a function of polymerization temperature, $T$, for quasi-stationary dodecyl acrylate solution polymerization initiated by laser pulses applied at a repetition rate of $20 \mathrm{~Hz}$, $c_{\mathrm{DA}}=0.92 \mathrm{~mol} \cdot \mathrm{L}^{-1}, c_{\mathrm{MMMP}}=6.5 \cdot 10^{-3} \mathrm{~mol} \cdot \mathrm{L}^{-1}$.

In comparison with $\mathrm{BA}$, the amount of mid-chain radicals in DA laser-initiated homopolymerization is much higher. Thus at $30{ }^{\circ} \mathrm{C}$ around $95 \%$ of the radicals in the reaction mixture are mid-chain ones. This can be explained as follows: In the polymerization of DA the long non-polar ester group may serve as an intramolecular diluent, as was pointed out by Beuermann [35], and thus the probability of the intramolecular chain transfer (backbiting) for DA is higher than for BA. 


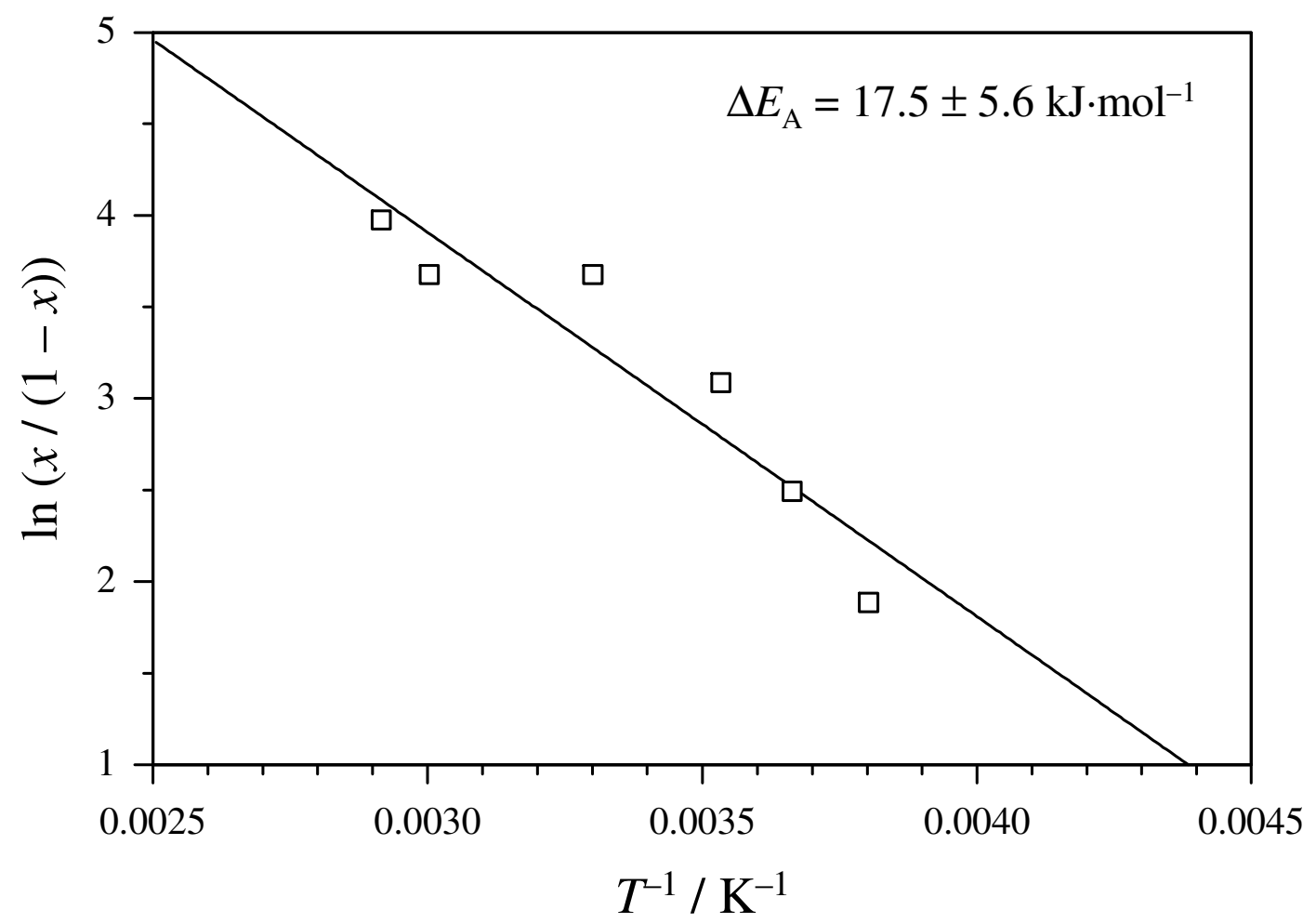

Fig. 7.14 Plot of $\ln (x /(1-x))$ vs. $T^{-1}$ for pulsed laser polymerizations of dodecyl acrylate, $c_{\mathrm{DA}}=0.92 \mathrm{~mol} \cdot \mathrm{L}^{-1}, c_{\mathrm{MMMP}}=6.5 \cdot 10^{-3} \mathrm{~mol} \cdot \mathrm{L}^{-1}$.

In Fig. 7.14, the data for DA laser initiated polymerization closely fits to a straight line. According to Eq. 7.2 the linear slope of the Arrhenius-type plot of $k_{\mathrm{bb}} / k_{\mathrm{p}} *$ in Fig. 7.14 should yield the difference between the activation energies of backbiting of an SPR species, $E_{\mathrm{A}}\left(k_{\mathrm{bb}}\right)$, and of the propagation reaction from an MCR1* species, $E_{\mathrm{A}}\left(k_{\mathrm{p}} *\right)$. The difference of activation energies is found to be: $\Delta E_{\mathrm{A}}=E_{\mathrm{A}}\left(k_{\mathrm{bb}}\right)-E_{\mathrm{A}}\left(k_{\mathrm{p}}{ }^{*}\right)=17.5 \pm 5.6 \mathrm{~kJ} \cdot \mathrm{mol}^{-1}$. With knowledge of $k_{\mathrm{p}}^{*}$, absolute numbers for $k_{\mathrm{bb}}$ may be estimated from the ESR-spectroscopically measured percentages of mid-chain radicals. 


\subsection{CONCLUSIONS}

In the present study, ESR experiments were carried out during $n$-butyl acrylate and dodecyl acrylate homopolymerizations in solution of toluene in a wide range of temperatures. Polymerization was induced by $351 \mathrm{~nm}$ excimer laser pulses applied at a repetition rate of $20 \mathrm{~Hz}$. The ESR spectra obtained under pulsed laser polymerization conditions at low degrees of monomer conversion, may be adequately represented by the superposition of a 4-line spectrum assigned to secondary propagating radicals (SPRs) and a 9-line spectrum assigned to tertiary mid-chain radicals (MCR1s), which latter radical results from backbiting of SPR species via a 1,5-H shift reaction. The percentage of MCR1 species is negligible at the lowest experimental temperature, but increases significantly with temperature. At ambient temperature, about 60 per cent of the stationary radical concentration in $n$-butyl acrylate polymerization and 90 per cent in dodecyl acrylate polymerization are MCR1 species. This observation explains the limitations for PLP-SEC experiments on $n$-butyl acrylate and dodecyl acrylate at temperatures of and above $30{ }^{\circ} \mathrm{C}$. The fraction of mid-chain radicals is highly relevant for modelling overall propagation, but also for understanding termination and transfer behavior in acrylate free-radical polymerizations. 


\subsection{REFERENCES}

[1] Olaj, O. F.; Bitai, I.; Henkelmann, F. Macromol. Chem. 1987, 188, 1689.

[2] Buback, M.; Gilbert, R. G.; Hutchinson, R. A.; Klumpermann, B.; Kuchta, F.-D.;Manders, B. G.; O’Driscoll, K. F.; Russell, G. T.; Schweer, J. Macromol. Chem. Phys. 1995, 196, 3267.

[3] Beuermann, S.; Buback, M.; Davis, T. P.; Gilbert, R. G.; Hutchinson, R. A.; Olaj, O. F.; Russell, G. T.; Schweer, J.; van Herk, A. M. Macromol. Chem. Phys. 1997, 198, 1545 .

[4] Beuermann, S.; Buback, M.; Davis, T. P.; Gilbert, R. G.; Hutchinson, R. A.; Kajiwara, A.; Klumpermann, B.; Russell, G. T. Macromol. Chem. Phys. 2000, 201, 1355.

[5] Beuermann, S.; Paquet, D. A.; McMinn Jr., J. H.; Hutchinson, R. A. Macromolecules, 1996, 29, 4206.

[6] Busch, M.; Wahl, A. Macromol. Thery Simul. 1998, 7, 217.

[7] Arzamendi, G.; Plessis, C.; Leiza, J. R.; Asua, J. M. Macromol. Theory Simul. 2003, 12,315 .

[8] Plessis, C.; Arzamendi, G.; Alberdi, J. M.; van Herk, A. M.; Leiza, J. R.; Asua, J. M. Macromol. Rapid. Commun. 2003, 24, 173.

[9] Plessis, C.; Arzamendi, G.; Leiza, J. R.; Schoonbrood, H. A. S.; Charmont, D.; Asua, J. M. Macromolecules 2000, 33, 4.

[10] Lyons, R.A.; Hutovic, J.; Piton, M. C.; Christie, D. I.; Clay, P. A.; Manders, B. G.; Kable, S. H.; Gilbert, R. G. Macromolecules 1996, 29, 1918.

[11] Ahmad, N. M.; Heatley, F.; Lovell, P. A. Macromolecules 1998, 31, 2822.

[12] Chiefari, J.; Jeffery, J.; Mayadunne, R. T. A.; Moad, G.; Rizzardo, E.; Thang, S. H. Macromolecules 1999, 32, 7700.

[13] van Herk, A. M. Macromol. Rapid Commun. 2001, 22, 687.

[14] Heatley, F.; Lovell, P. A.; Yamashita, T. Macromolecules 2001, 34, 7636.

[15] Britton, D.; Heatley, F.; Lovell, P. A. Macromolecules 1998, 31, 2828.

[16] Toh, J. S. S.; Huang, D. M.; Lovell, P. A.; Gilbert, R. G. Polymer 2001, 42, 1915.

[17] Asua, J. M.; Beuermann, S.; Buback, M.; Castignolles, P.; Charleux, B.; Gilbert, R. G.; Hutchinson, R. A.; Leiza, J. R.; Nikitin, A. N.; Vairon, J. P.; Herk, A. M. v. Macromol. Chem. Phys. 2004, 205, 2151.

[18] Müller, M. Ph. D. Thesis, Göttingen, 2005. 
[19] Tanaka, K.; Yamada, B.; Fellows, C. M.; Gilbert, R. G.; Davis, T. P.; Yee, L. H.; Smith, G. B.; Rees; M. T. L.; Russell; G. T. J. Polym. Chem. 2001, 39, 3902.

[20] Kajiwara, A.; Kamachi, M. ACS Symposium Series 2003, 854 (Advances in Controlled/Living Radical Polymerization), 86.

[21] Fischer, H. Z. Naturforsh. 1964, 19a, 267, 866.

[22] Kamachi, M. Adv. Polym. Sci. 1987, 82, 207.

[23] Fischer, H.; Giacometti, G. J. Polym. Sci.: C 1967, 16, 2763.

[24] Best, M. E.; Kasai, P. H. Macromolecules 1989, 22, 2622.

[25] Gilbert, B. G.; Lindsay Smith, J. R.; Milne, E. C.; Whitwood, A. C.; Taylor, P. J. Chem. Soc., Perkin Trans. 2 1993, 2025.

[26] Doetschmann, D. C.; Mehlenbacher, R. C. M.; Cywar, D. Macromolecules, 1996, 29, 1807.

[27] Azukizawa, M.; Yamada, B.; Hill, D. J. T.; Pomery, P. J. Macromol. Chem. Phys. 2000, 201,774 .

[28] Yamada, B.; Azukizawa, M.; Yamazoe, H.; Hill, D. J. T.; Pomery, P. J. Polymer, 2000, $41,5611$.

[29] Chang, H. R.; Lau, W.; Parker, H.-Y.; Westmoreland, D. G.; Macromol. Symp. 1996, $111,253$.

[30] Kloosterboer, J. G.; Lijten, G. F. C. M.; Greidanus, F. J. A. M. Polymer Communications 1986, 27, 268.

[31] Kim, S. S.; Liang, R. H.; Tsay, F. D.; Gupta, A. Macromolecules 1986, 19, 1930.

[32] Tanaka, K.; Yamada, B.; Willemse, R.; van Herk, A. M. Polymer Journal (Tokyo, Japan) 2002, 34 (9), 692.

[33] Sato, E.; Emoto, T.; Zetterlund, P. B.; Yamada, B. Macromol. Chem. Phys. 2004, 205 (14), 1829.

[34] Kajiwara, A.; Nanda, A. K.; Matyjaszewski, K. Macromolecules 2004, 37, 1378.

[35] Beuermann, S. Macromol. Symp. 2002, 182, 31. 


\section{Determination of Propagation Rate Coefficients, $k_{\mathrm{p}}$, FOR THE DODECYL ACRYLATE DIMER (DAD)}

\subsection{INTRODUCTION}

Resent studies have shown that transfer reactions (intra- and intermolecular transfer to polymer) play an important role in the polymerization of acrylates at high and moderately high (above $30^{\circ} \mathrm{C}$ ) temperatures [1,2]. Via these reactions, secondary propagating radicals are transferred into tertiary mid-chain radicals. The most significant effect on acrylate polymerization has the intramolecular transfer reaction (backbiting). In this process the secondary propagating radical wraps around to abstract a hydrogen atom from an acrylate unit on its own backbone, most likely via the formation of a six-membered rind. The occurrence of mid-chain radicals, produced by transfer-to-polymer processes, has been confirmed by electron spin resonance (ESR) spectroscopy under stationary free-radical polymerization conditions chiefly carried out by Yamada and colleagues [3,4,5], Kajiwara and Kamachi [6] and by the observation of quaternary carbon atoms with ${ }^{13} \mathrm{C}-\mathrm{NMR}$ [1] under PLP-conditions. ESR-PLP experiments, presented in Section 7, show that the backbiting event must be considered during acrylate polymerization even at low temperature PLP experimental conditions. PLP studies on $n$-butyl acrylate (BA) demonstrate that addition of monomer to a secondary propagating radical is very fast $[7,8]$, but observed rates of BA polymerization are significantly lower than would be expected from estimate $[9,10]$. This result is in agreement with evidence suggesting that monomer addition to the mid-chain radical proceeds at a much slower rate than addition to the parent secondary radical. Propagating rate of mid-chain radicals has been estimated via the kinetics of the sterically hindered methyl acrylate dimer (MAD) [11] and butyl acrylate dimer (BAD) [12] (species that form radicals of similar structure to the mid-chain radicals). It is apparent that the problems found in obtaining data that obey the necessary consistency tests when applying PLP to acrylates might be found in PLP studies on DAD. Thus investigations into the propagation kinetics of the dodecyl acrylate dimer may assist in a better understanding of the underlying processes taking place in freeradical polymerization of acrylates.

The structure of the dodecyl acrylate dimer (DAD) is given in Fig. 8.1. 


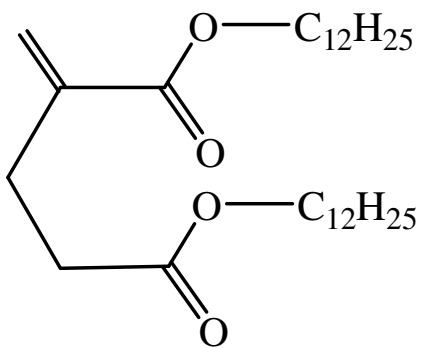

Fig. 8.1 Structure of the dodecyl acrylate dimer (DAD).

The goal of the present study was to determine the propagation rate coefficients, $k_{\mathrm{p}}$, of DAD by the PLP-SEC technique.

\subsection{EXPERIMENTAL RESULTS}

In this section, $k_{\mathrm{p}}$ values resulting from PLP-SEC experiments at ambient pressure will be provided. Fig. 8.2 presents a MWD for a DAD polymer obtained from a bulk polymerization at $60{ }^{\circ} \mathrm{C}$ and at ambient pressure at a laser-pulse repetition rate of $5 \mathrm{~Hz}$. The dashed line in Fig. 8.2 represents the first derivative curve of the MWD, which shows two well-resolved maxima corresponding to the points of inflection of the MWD, indicated by $\mathrm{L}_{1}$ and $\mathrm{L}_{2}$. The position of the second inflection point $\left(\mathrm{L}_{2}\right)$ is located at around twice the molecular weight of the first inflection point $\left(\mathrm{L}_{1}\right)$. 


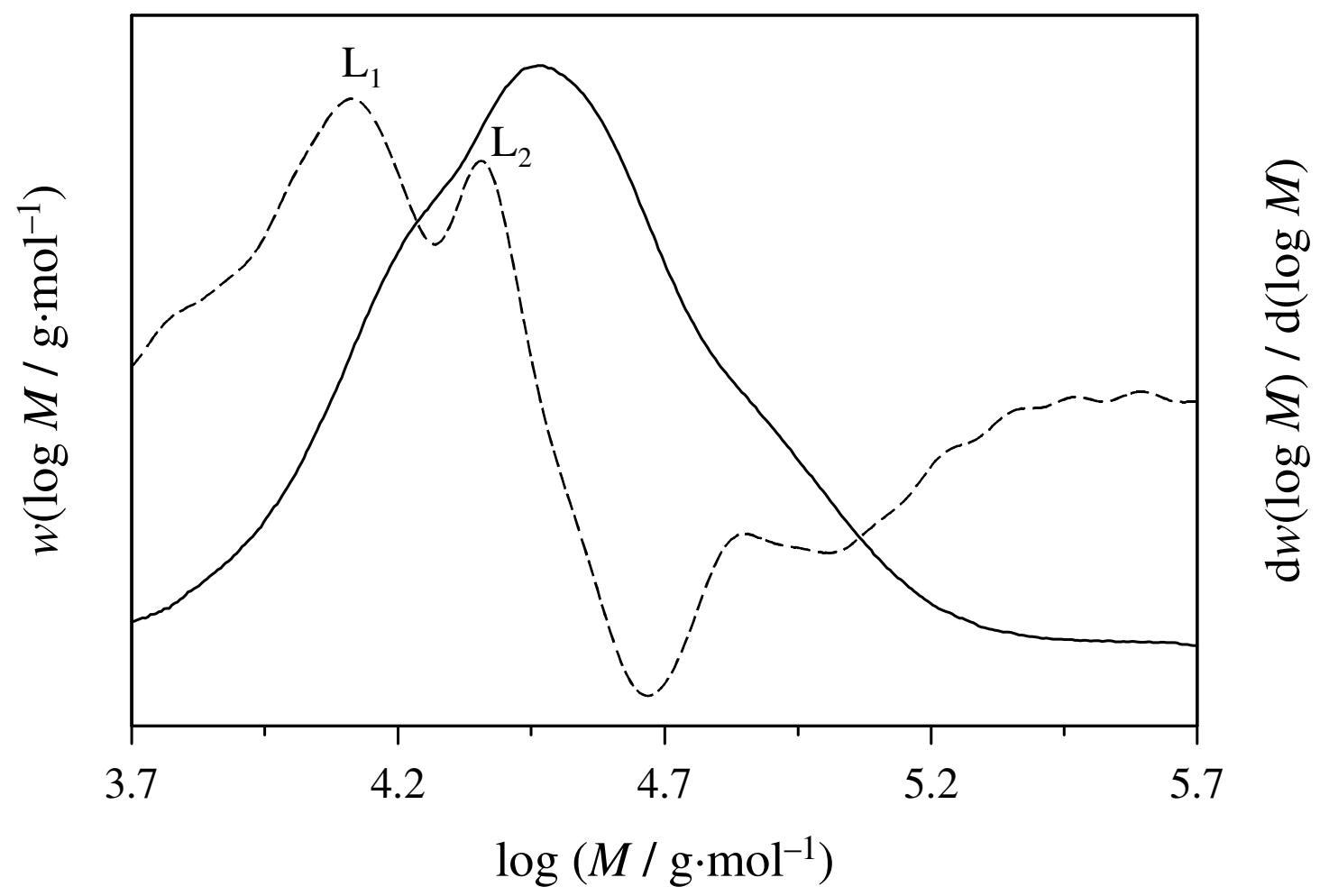

Fig. 8.2 Experimental molecular weight distribution (full line) of a DAD polymer produced at $60{ }^{\circ} \mathrm{C}$ and ambient pressure using a pulse repetition rate of $5 \mathrm{~Hz}$. The first derivative of the MWD is also given (dashed line).

\subsubsection{Estimation OF MARK-HouWink Parameters}

In order to determine propagation rate coefficients via PLP-SEC, reliable Mark-Houwink parameters for SEC calibration are required. Unfortunately, no literature data of these values for poly(DAD) have been reported. Mark-Houwink parameters for poly(MAD) [11] and poly(BAD) [12] are available. In Tab. 8.1 values of the Mark-Houwink parameters, $K$ and $a$, for MAD and $\mathrm{BAD}$ are given together with the values for poly(methyl acrylate (MA)), poly(butyl acrylate (BA)), poly(dodecyl acrylate (DA)), poly(methyl methacrylate (MMA)), poly(butyl methacrylate (BMA)), poly(dodecyl methacrylate (DMA)), as well with poly(dicyclohexyl itaconate (DCHI)), and poly(dimethyl itaconate (DMI)). 


\begin{tabular}{cccc}
\hline Monomer & $a$ & $K / \mathrm{mL} \cdot \mathrm{g}^{-1}$ & Source \\
\hline MA & 0.696 & 0.0168 & {$[13]$} \\
BA & 0.700 & 0.0122 & {$[14]$} \\
DA & 0.585 & 0.0292 & {$[15]$} \\
MMA & 0.719 & 0.00944 & {$[16]$} \\
BMA & 0.664 & 0.0148 & {$[17]$} \\
DMA & 0.736 & 0.00495 & {$[13]$} \\
MAD & 0.820 & 0.0028 & {$[11]$} \\
BAD & 0.624 & 0.0187 & {$[12]$} \\
DCHI & 0.580 & 0.0233 & {$[18]$} \\
DMI & 0.510 & 0.0460 & {$[19]$} \\
\hline
\end{tabular}

Tab. 8.1 Mark-Houwink parameters, $K$ and $a$, for a series of poly(acrylates) and poly(methacrylates), as well as for poly(dicyclohexyl itaconate (DCHI)) and poly(dimethyl itaconate (DMI)) in THF.

The Mark-Houwink parameters are always determined as coupled parameters according to the Mark-Houwink relation Eq. 3.16 [20,21]. Therefore it is possible to describe the intrinsic viscosity, $[\eta]$, within a certain range of molecular weights with different sets of MarkHouwink parameters. Thus in order to try to identify trends in the Mark-Houwink parameters for acrylates, methacrylates and itaconates, the connection of intrinsic viscosity versus molecular weight of polymers is given in Fig. 8.3. The data are obtained using the $K$ and $a$ values listed in Tab. 8.1. 


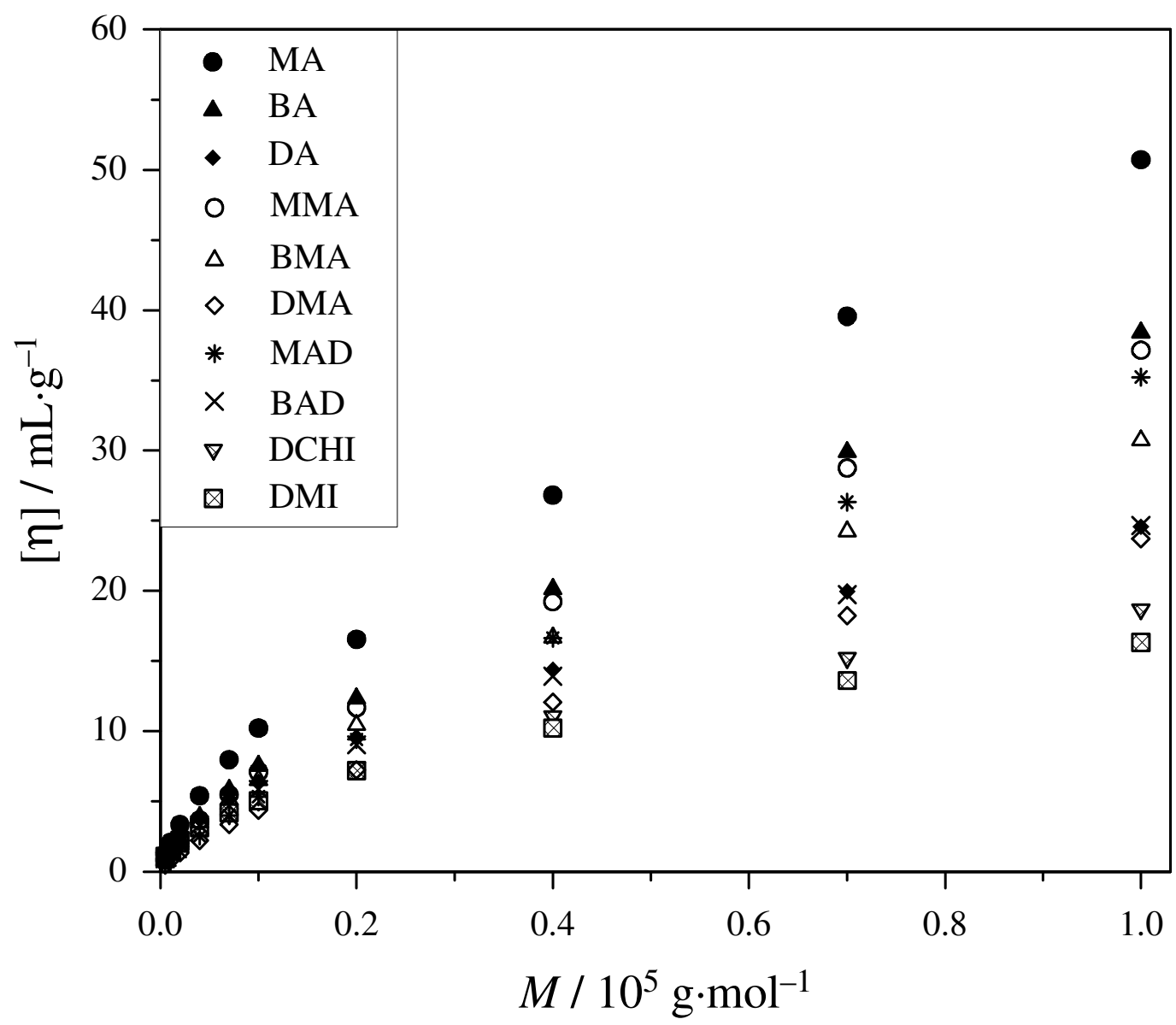

Fig. 8.3 Comparison of intrinsic viscosity, $[\eta]$, of different (meth)acrylates, itaconates, $\mathrm{MAD}$, and BAD at the same molecular masses.

As can be seen from Fig. 8.3, the intrinsic viscosity for acrylates is higher than for methacrylates and acrylate dimers and increases as follows for the monomers with the same

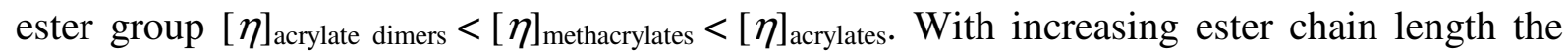
intrinsic viscosity decreases in the following order $[\eta]_{\text {methyl }}>[\eta]_{\text {butyl }}>[\eta]_{\text {dodecyl }}$. It is interesting to compare the intrinsic viscosity of itaconates with $[\eta]$-values of acrylates and methacrylates because the structure of itaconates (Fig. 8.4) bears a resemblance to the structure of acrylate dimers with one difference: acrylate dimers have one $\mathrm{CH}_{2}$ group more in the backbone as itaconates. 

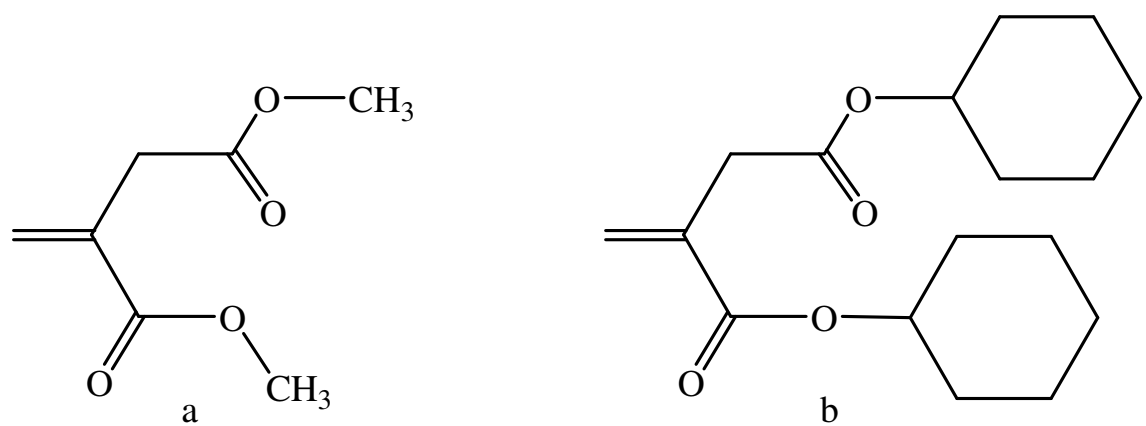

Fig. 8.4 The structure of dimethyl itaconate (a) and dicyclohexyl itaconate (b).

The values of intrinsic viscosity of DMI and DCHI differ not that much from each other (Fig. 8.3). In order to estimate propagation rate coefficients of DAD the Mark-Houwink parameters of DCHI were chosen because this monomer has rather bulky ester chains just as DAD and $[\eta]_{\mathrm{DCHI}}$ is less than $[\eta]_{\mathrm{DMA}}$, what one would expect from the observations made for MAD and BAD.

\subsubsection{DETERMINATION OF $k_{\mathrm{p}}$ VALUES}

Applying the PLP-SEC technique, the propagation rate coefficient is derived from the molecular weight distribution according to Eq. 3.12.

The density of DAD was determined as a function of temperature. The data obey the following equation over the temperature range 20 to $70{ }^{\circ} \mathrm{C}$ :

$$
\rho_{\mathrm{DAD}} /\left(\mathrm{g} \cdot \mathrm{mL}^{-1}\right)=-7.91 \cdot 10^{-4} \cdot \Theta /{ }^{\circ} \mathrm{C}+0.922
$$

The $k_{\mathrm{p}}$ data for DAD bulk polymerization obtained from the PLP-SEC experiments (together with a laser pulse repetition rate and the inflection points used for $k_{\mathrm{p}}$ determination) are listed in Tab. 8.2. Absolute values of $k_{\mathrm{p}}$ are not easily deduced from PLP-SEC, as Mark-Houwink parameters are not available for poly(DAD) in THF, which is the standard solvent for SEC analyses. In view of the uncertainties associated with the conventional procedures of $k_{\mathrm{p}}$ determination [22,23], but also with using approximate Mark-Houwink coefficients for deducing poly(DAD) molecular weights, the agreement between $k_{\mathrm{p}}$ values of $\mathrm{DAD}$ and the literature data for MAD and BAD appears to be satisfactory (see Tab. 8.3). 


\begin{tabular}{ccccc}
\hline$\Theta /{ }^{\circ} \mathrm{C}$ & $v / \mathrm{Hz}$ & $M_{\mathrm{L} 1} / \mathrm{g} \mathrm{mol}^{-1}$ & $M_{\mathrm{L} 2} / \mathrm{g} \mathrm{mol}^{-1}$ & $k_{\mathrm{p}} / \mathrm{L} \cdot \mathrm{mol}^{-1} \cdot \mathrm{s}^{-1}$ \\
\hline 20 & 1 & 10805 & 19785 & 16.9 \\
20 & 1 & 10324 & 19151 & 16.1 \\
30 & 1 & 16167 & 27108 & 26.4 \\
30 & 5 & 5333 & 9987 & 39.5 \\
30 & 5 & 5311 & 10232 & 39.4 \\
40 & 5 & 6491 & 12724 & 49.4 \\
40 & 5 & 6960 & 13239 & 53.3 \\
40 & 10 & 4936 & 9418 & 73.3 \\
50 & 5 & 10306 & 18735 & 82.3 \\
50 & 5 & 10300 & 19106 & 82.2 \\
60 & 5 & 12747 & 22285 & 104.6 \\
60 & 5 & 12825 & 22266 & 105.3 \\
70 & 5 & 16645 & 26915 & 141.0 \\
70 & 5 & 16526 & 26303 & 139.9 \\
70 & 10 & 10637 & 18430 & 173.5 \\
70 & 10 & 10587 & 18091 & 172.6 \\
80 & 16 & 9806 & 18430 & 256.4 \\
\hline
\end{tabular}

Tab. 8.2 PLP-SEC results for the bulk polymerization of DAD obtained at ambient pressure .

The temperature dependence of $k_{\mathrm{p}}$ for the data from Tab. 8.2 is shown in an Arrhenius diagram in Fig. 8.5. All data are nicely represented by a single linear fit over the entire temperature range, given by the full line in Fig. 8.5. The following Arrhenius relation for $20{ }^{\circ} \mathrm{C} \leq \Theta \leq 80{ }^{\circ} \mathrm{C}$ is obtained:

$$
\ln \left[k_{\mathrm{p}} /\left(\mathrm{L} \cdot \mathrm{mol}^{-1} \cdot \mathrm{s}^{-1}\right)\right]=17.53-4261 \cdot\left(T^{-1} / \mathrm{K}^{-1}\right)
$$

This equation corresponds to an activation energy of $E_{\mathrm{A}}=35.4 \mathrm{~kJ} \cdot \mathrm{mol}^{-1}$. The pre-exponential factor for DAD is obtained to be $4.10 \cdot 10^{7} \mathrm{~L} \cdot \mathrm{mol}^{-1} \cdot \mathrm{s}^{-1}$. 


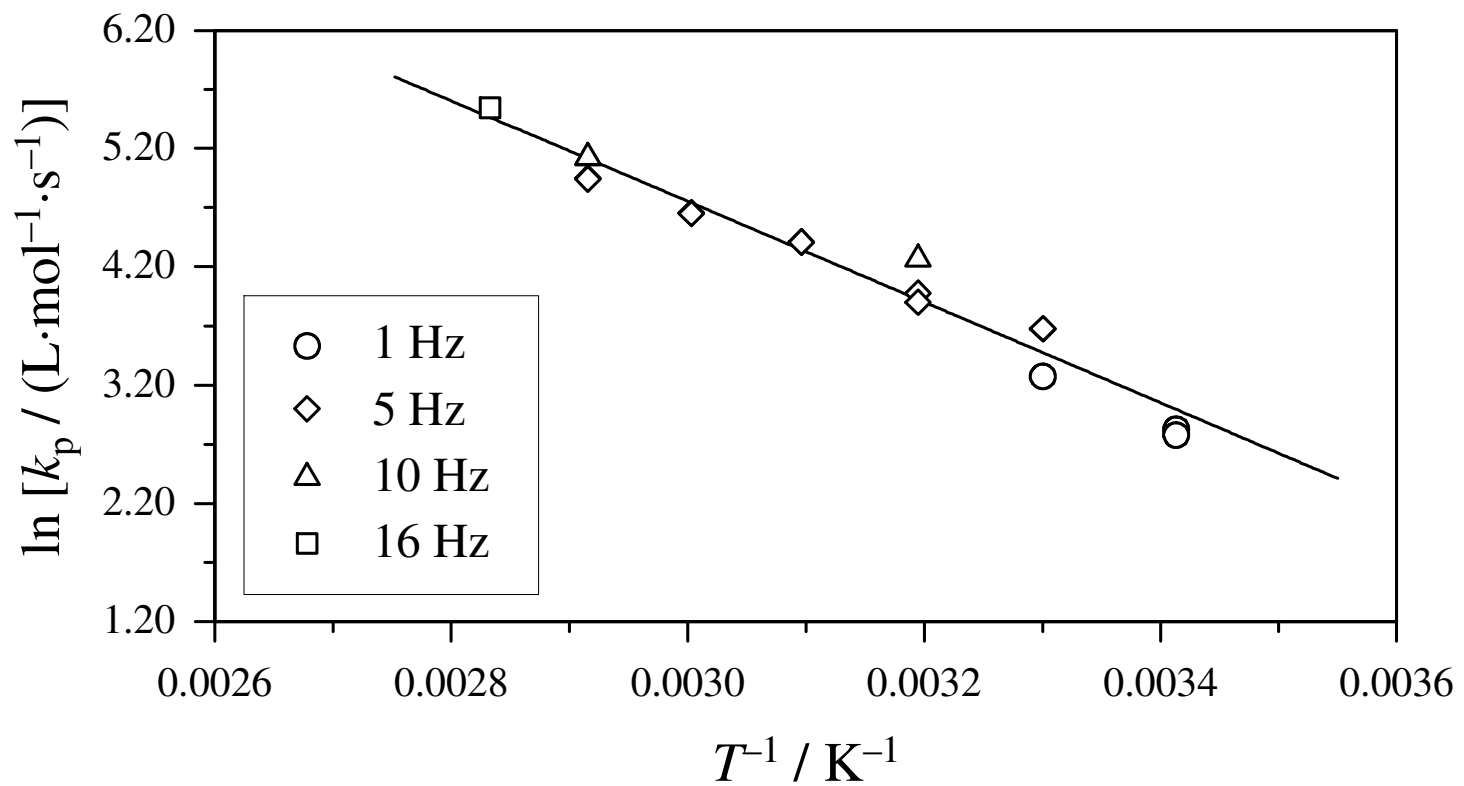

Fig. 8.5 Arrhenius plot of $k_{\mathrm{p}}$ for DAD from the PLP-SEC experiments. The line represents the best fit of the PLP-SEC data by Eq. 8.1.

Tab. 8.3 contains the Arrhenius parameters for some (meth)acrylates, itaconates, MAD and BAD.

\begin{tabular}{ccccc}
\hline Monomer & $A \cdot 10^{-6} / \mathrm{L} \cdot \mathrm{mol}^{-1} \cdot \mathrm{s}^{-1}$ & $E_{\mathrm{A}} / \mathrm{kJ} \cdot \mathrm{mol}^{-1}$ & $k_{\mathrm{p}} / \mathrm{L} \cdot \mathrm{mol}^{-1} \cdot \mathrm{s}^{-1}$ & Source \\
\hline MA & 16.6 & 17.7 & $11600^{*}$ & {$[24]$} \\
BA & 18.1 & 17.4 & $14300^{*}$ & {$[25,26]$} \\
DA & 17.9 & 17.0 & $16700^{*}$ & {$[15]$} \\
MMA & 2.7 & 22.4 & 491 & {$[27]$} \\
BMA & 3.8 & 22.9 & 570 & {$[16]$} \\
DMA & 2.5 & 21.0 & 779 & {$[16]$} \\
DCHI & 0.02 & 22.0 & 4 & {$[28]$} \\
DMI & 0.2 & 24.9 & 14 & {$[19]$} \\
MAD & 3.4 & 33.6 & 8 & {$[29]$} \\
MAD & 1.3 & 29.5 & 15 & {$[11]$} \\
BAD & 9.2 & 33.8 & 21 & {$[12]$} \\
DAD & 41.0 & 35.4 & 50 & this work \\
\hline
\end{tabular}

Tab. 8.3 Arrhenius parameters $A$ and $E_{\mathrm{A}}$ of $k_{\mathrm{p}}$ for some acrylates, methacrylates, itaconates, MAD and BAD. All data refers to $40{ }^{\circ} \mathrm{C}$ (except acrylates) and ambient pressure. $k_{\mathrm{p}}$ is calculated using the given Arrhenius parameters. 
Tab. 8.3 shows some clear trends in $E_{\mathrm{A}}$, for example, acrylates have $E_{\mathrm{A}} \sim 17-18 \mathrm{~kJ} \cdot \mathrm{mol}^{-1}$, whereas for methacrylates, the corresponding value is $\sim 21-23 \mathrm{~kJ} \cdot \mathrm{mol}^{-1}$, for itaconates $E_{\mathrm{A}} \sim 24 \mathrm{~kJ} \cdot \mathrm{mol}^{-1}$ and for acrylate dimers $E_{\mathrm{A}} \sim 34 \mathrm{~kJ} \cdot \mathrm{mol}^{-1}$. Extensive quantum mechanical calculations of the transition stats for acrylonitrile and methacrylonitrile [30] have shown the reasons for the approximate values for the frequency factors of the acrylate and methacrylate systems, as well as for the systematic differences in both frequency factor and activation energy between the two monomer types. The typical values of $A$ for these systems are determined by the simple parameters governing the partition function for translation and external rotation (mass and geometry, respectively), combined with typical values for the partition functions for the modes that are vibrations in the reactant but hindered rotors in the transition state. The lower frequency factors for methacrylates are largely due to hindrance caused by the methyl groups to the three transitional modes in the transition state that correspond to the three external rotational degrees of freedom of the monomer in the reactants (but not a result of increased hindrance to methyl rotation itself in the transition state). The higher activation energy of methacrylates arise from differences in hindrance and loss of delocalization in the transition states of the methyl-substituted and unsubstituted monomers.

In comparison with methacrylates acrylate dimers are more hindered. It would not be unreasonable to assume that hindrance would raise the activation energy above that of a monomeric acrylate or methacrylate.

It should be noted, that the substituent that is introduced into the ethene molecule facilitates stabilization of the propagating radical and has the effect of adding steric hindrance to the propagating step. As can be seen from Tab. 8.3, side groups in the $\alpha$-position have a much greater impact on the propagation rate of a monomer in free-radical polymerization than ester substitutions because of the closer proximity to the propagating free-radical center. Steric effects acquire much importance when the substituent is placed rather close to the reactive center. If one changes the size of an ester, a drastic effect on the propagation rate is difficult to conceive. The reactive center in this case is located several bonds apart.

The data in Tab. 8.3 clearly shows that $k_{\mathrm{p}}$ increases with the ester size within a monomer family. Beuermann [31] pointed out that in PLP-SEC experiments the product of $k_{\mathrm{p}}$ and $c_{\mathrm{M}}$ is measured: $k_{\mathrm{p}} \cdot c_{\mathrm{M}}=L_{1} / t_{0}$. As a consequence, variations of $k_{\mathrm{p}}$ are implied, if $c_{\mathrm{M}}$ at the site of the propagation reaction is not identical with the overall or analytical monomer concentration $c_{\mathrm{M}}$ in the system. In order to clarify the situation the analytical or overall monomer concentration is defined as $c_{\mathrm{M}, \mathrm{a}}$, the experimentally derived apparent propagating rate coefficient as $k_{\mathrm{p}, \exp }$, and the true kinetic propagation rate coefficient as $k_{\mathrm{p}, \mathrm{kin}}$. The relation 
between monomer concentrations and propagation rate coefficients are as follows: $L_{1} / t_{0}=k_{\mathrm{p}, \mathrm{kin}} \cdot c_{\mathrm{M}, \mathrm{loc}}=k_{\mathrm{p}, \exp } \cdot c_{\mathrm{M}, \mathrm{a}}$.

In case of monomers with the methyl ester group strong intramolecular interactions between polymer segments may occur. These interactions are expected to result in an effective displacement of a large fraction of monomer molecules from the surroundings of the freeradical chain and thus the occurrence of a local monomer concentration, $c_{\mathrm{M}, \text { loc }}$, which is below the total monomer concentration in the system. In polymerizations of monomers with the dodecyl ester group the long non-polar ester group may serve as an intermolecular diluent reducing the polar interactions between polymer segments due to shielding. The local monomer concentration in the vicinity of the propagating chain-end is closer to the overall monomer concentration in the system. Thus, the occurrence of the local monomer concentration may (at least partly) explain the observed increase of $k_{\mathrm{p}}$ with ester size within the acrylates, within the methacrylates and within the acrylate dimers family.

\subsection{CONCLUSIONS}

The commonly used PLP-SEC technique was applied for the measurement of propagation rate coefficients of the highly hindered dodecyl acrylate dimer. The activation energy of the propagation reaction is $E_{\mathrm{A}}=35.4 \pm 4.3 \mathrm{~kJ} \cdot \mathrm{mol}^{-1}$. This value is in good agreement with previously determined values of $E_{\mathrm{A}}$ for the methyl acrylate dimer and the butyl acrylate dimer. 


\subsection{REFERENCES}

[1] Plessis, C.; Arzamendi, G.; Alberdi, J. M.; van Herk, A. M.; Leiza, J. R.; Asua, J. M. Macromol. Rapid. Commun. 2003, 24, 173.

[2] Arzamendi, G.; Plessis, C.; Leiza, J. R.; Asua, J. M. Macromol. Theory Simul. 2003, 12,315 .

[3] Azukizawa, M.; Yamada, B.; Hill, D. J. T.; Pomery, P. J. Macromol. Chem. Phys. 2000, 201, 774 .

[4] Yamada, B.; Azukizawa, M.; Yamazoe, H.; Hill, D. J. T.; Pomery, P. J. Polymer 2000, 41,5611 .

[5] Sato, E.; Emoto, T.; Zetterlund, P. B.; Yamada, B. Macromol. Chem. Phys. 2004, 205 (14), 1829.

[6] Kajiwara, A.; Kamachi, M. ACS Symposium Series 2003, 854 (Advances in Controlled/Living Radical Polymerization), 86.

[7] Beuermann, S.; Paquet Jr., D. A.; McMinn, J. H.; Hutchinson, R. A. Macromolecules 1996, 29, 4206.

[8] Asua, J. M.; Beuermann, S.; Buback, M.; Castignolles, P.; Charleux, B.; Gilbert, R. G.; Hutchinson, R. A.; Leiza, J. R.; Nikitin, A. N.; Vairon, J. P.; Herk, A. M. v. Macromol. Chem. Phys. 2004, 205, 2151.

[9] Plessis, C.; Arzamendi, G.; Leiza, J. R.; Schoonbrood, H. A. S.; Charmot, D.; Asua, J. M. Macromolecules 2000, 33, 4.

[10] Peck, A. N. F.; Hutchinson, R. A. Macromolecules 2004, 37, 5944.

[11] Tanaka, K.; Yamada, B.; Fellows, C. M.; Gilbert, R. G.; Davis, T. P.; Yee, L. H.; Smith, G. B.; Rees, M. T. L.; Russell, G. T. J. Polym. Sci. Part A, Polym. Chem. 2001, $39,3902$.

[12] Müller, M. Ph. D. Thesis, Göttingen, 2005.

[13] Buback, M.; Feldermann, A.; Barner-Kowollik, C.; Lacík, I. Macromolecules 2001, $34,5439$.

[14] Hutchinson, R. A.; McMinn, J. H.; Paquet Jr., D. A.; Beuermann, S.; Jackson, C. Ind. Eng. Chem. Res. 1997, 36, 1103.

[15] Buback, M.; Kurz, C. H.; Schmaltz, C. Macromol. Chem. Phys. 1998, 199, 1721.

[16] Beuermann, S.; Buback, M.; Davis, T. P.; Gilbert, R. G.; Hutchinson, R. A.; Kajiwara, A.; Klumperman, B.; Russell, G. T. Macromol. Chem. Phys. 2000, 201, 1355. 
[17] Hutchinson, R. A.; Beuermann, S.; Paquet Jr., D. A.; McMinn, J. H. Macromoleciles 1997, 30, 3451 .

[18] Velickovic, J.; Coseva, S.; Fort, R. J. Eur. Polym. J. 1975, 11, 377.

[19] Yee, L. H.; Coote, M. L.; Chaplin, R. P.; Davis, T. P. J. Polym. Sci. Part A, Polym. Chem. 2000, 38, 2192.

[20] Mark, H. in 'Der feste Körper', Hirzel, Leipzig,. 1938, 65.

[21] Houwink, R. J. Prakt. Chem. 1940, 157,15.

[22] Jackson, C.; Chen, Y.-J.; Mays, J. W. J. Appl. Polym. Sci. 1996, 61, 865.

[23] Coote, M. L.; Davis, T. P. J. Polym. Sci., Part B : Polym. Phys. 1999, 37, 2557.

[24] Manders, B. G. Ph. D. Thesis, Eindhoven, 1997.

[25] Beuermann, S.; Paquet Jr., D. A.; McMinn, J. H.; Hutchinson, R. A. Macromolecules 1996, 29, 4206.

[26] Lyons, R. A.; Hutovic, J.; Piton, M. C.; Christie, D. I.; Clay, P. A.; Manders, B. G.; Kable, S. H.; Gilbert, R. G. Macromolecules 1996, 29, 1918.

[27] Beuermann, S.; Buback, M.; Davis, T. P.; Gilbert, R. G.; Hutchinson, R. A.; Olaj, O. F.; Russell, G. T.; Schweer, J.; van Herk, A. M. Macromol. Chem. Phys. 1997, 198, 1545.

[28] Vana, P.; Yee, L. H.; Davis. T. P. Macromolecules 2002, 35, 3008.

[29] Kobatake, S.; Yamada, B. J. Polym. Sci. Part A: Polym. Chem. 1996, 34, 95.

[30] Huang, D. M.; Monteiro, M. J.; Gilbert, R. G. Macromolecules 1998, 31, 5175.

[31] Beuermann, S. Macromol. Symp. 2002, 182, 31. 


\section{Chemically Initiated Polymerization}

\subsection{INTRODUCTION}

In spite of much attention that has been given to free-radical polymerizations, there are still many aspects that are not fully understood. Particularly difficult is the determination of propagation rate coefficients by PLP-SEC for acrylate monomers at temperatures above $30{ }^{\circ} \mathrm{C}$ due to backbiting reactions, as has been pointed out in Section 7. Coupled parameter $k_{\mathrm{p}} / k_{\mathrm{t}}^{0.5}$ is easier to be obtained, e.g. via simple techniques based on conversion measurements in which the rate of initiation changes only slowly with time, for example by employing a thermally decomposing chemical initiator. Such experiments provide the rate of polymerization over relatively small time intervals. From each rate measurement, $k_{\mathrm{p}} / k_{\mathrm{t}}^{0.5}$ can be determined, where each $k_{\mathrm{t}}$ is a chain-length average value, $\left\langle k_{\mathrm{t}}>\right.$ (see Section 6.2).

A general expression for the rate of stationary polymerization is given below:

$$
R_{\mathrm{p}}=-\frac{\mathrm{d} c_{\mathrm{M}}}{\mathrm{d} t}=\frac{k_{\mathrm{p}}}{\sqrt{k_{\mathrm{t}}}} \cdot c_{\mathrm{M}} \cdot \sqrt{f \cdot k_{\mathrm{d}} \cdot c_{\mathrm{I}}}
$$

This expression correlates the rate of polymerization with the initiator and monomer concentrations on the one side and the rate coefficients $k_{\mathrm{d}}, k_{\mathrm{p}}$, and $k_{\mathrm{t}}$ on the other side (see also Section 3). Eq. 9.1 indicates a first-order dependence of the rate of polymerization on the monomer concentration and a square-root dependence on the concentration of the initiator.

In general, for acrylic acid esters it has been found that acrylate kinetics deviates considerably from the expected behavior for stationary free-radical polymerization. Numerous studies $[1,2,3,4,5,6,7,8,9]$ indicate that for acrylates the reaction order with respect to monomer concentration is higher than unity (Tab.9.1), whereas, in accordance with the theory, a reaction order of 0.5 is normally found with respect to initiator concentration.

Methacrylates, on the other hand, have monomer reaction orders close to the theoretically predicted value of unity. 


\begin{tabular}{cccccc}
\hline Monomer & $\Theta /{ }^{\circ} \mathrm{C}$ & Initiator & Solvent & $\begin{array}{c}\text { Order of } \\
\text { monomer }\end{array}$ & Source \\
\hline MA & 50 & lauryl peroxide & toluene & 1.5 & {$[10]$} \\
BA & 40 & AIBN & ethyl acetate & 1.5 & {$[3]$} \\
BA & 50 & lauryl peroxide & toluene & 1.5 & {$[10]$} \\
BA & 50 & AIBN & benzene & 1.5 & {$[9]$} \\
DA & 40 & AIBN & ethyl acetate & 1.6 & {$[1]$} \\
DA & 40 & AIBN & heptane & 1.6 & {$[1]$} \\
\hline MMA & 50 & lauryl peroxide & toluene & 1.0 & {$[10]$} \\
MMA & 60 & AIBN & benzene & 1.0 & {$[11]$} \\
BMA & 60 & AIBN & benzene & 1.0 & {$[11]$} \\
\hline
\end{tabular}

Tab. 9.1 Reaction order with respect to monomer concentration for some acrylates and methacrylates at different temperatures and in different solvents.

The progress in the field of free-radical polymerization modeling is essentially part of the availability of efficient mathematical tolls like PREDICI ${ }^{\circledR}$. This program e.g. allows integrating systems of countable differential equations as they result for polymerization scheme without any assumptions with respect to closure conditions and calculate full MWDs for almost any type of polymerization experiments. All simulations in this study are performed using PREDICI ${ }^{\circledR}$. In order to simplify the analyzing procedure, chain-length- and conversionindependent rate coefficients $k_{\mathrm{p}}$ and $k_{\mathrm{t}}$ were considered.

The purpose of this section is to model steady-state polymerizations initiated by a thermally decomposing chemical initiator and to provide experimental data for testing the model.

The present study is focused on the steady-state free-radical polymerization of butyl acrylate (BA), dodecyl acrylate (DA) and dodecyl methacrylate (DMA) at different temperatures, initiator and monomer concentrations. All experiments were carried out at ambient pressure.

\subsection{Model DeVElopment}

A basic model of free-radical polymerization takes into account the set of reactions denoted in section 3.1. For acrylate polymerization it is necessary to consider the generation of tertiary (mid-chain) radicals via intramolecular transfer to polymer. In the present work it will be assumed that the radical site can be transferred to any position within the chain (not only to a position close to the chain end) or to any position within one of the ester side chains. The considered reaction will, therefore, be referred to as intramolecular transfer to polymer (which includes but is not limited to backbiting). Other transfer reactions, such as intermolecular transfer to polymer or transfer to monomer, will be neglected, as their influence is less 
significant as compared to intramolecular transfer reaction. The reaction of $\beta$-scission has been found to be of negligible importance at temperatures below $100{ }^{\circ} \mathrm{C}$ and hence was not considered in the kinetic scheme.

It has recently been shown that acrylates may terminate by disproportionation reactions [12]. However, neither the relative importance of this mechanism with respect to the combination mechanism nor its significance at higher temperatures (the data was determined at $-34{ }^{\circ} \mathrm{C}$ ) is given. Therefore, in this work bimolecular termination was considered to occur exclusively by combination [13].

The following kinetic scheme is considered:

Initiation:

formation of primary radicals:

formation of secondary radicals:

Propagation of secondary radicals:

Intramolecular transfer:

Propagation of tertiary radicals:

Termination

$$
\begin{aligned}
& \text { of secondary radicals: } \\
& \text { cross-termination: } \\
& \text { of tertiary radicals: }
\end{aligned}
$$

$$
\begin{aligned}
& \mathrm{I} \stackrel{k_{\mathrm{d}}}{\longrightarrow} 2 \mathrm{R}_{1}^{*} \\
& \mathrm{R}_{1}^{*}+\mathrm{M} \stackrel{k_{\mathrm{p} 1}}{\longrightarrow} \mathrm{R}_{1} \\
& \mathrm{R}_{1}+\mathrm{M} \stackrel{k_{\mathrm{p} 1}}{\longrightarrow} \mathrm{R}_{1} \\
& \mathrm{R}_{1} \stackrel{k_{\mathrm{bb}}}{\longrightarrow} \mathrm{R}_{2} \\
& \mathrm{R}_{2}+\mathrm{M} \stackrel{k_{\mathrm{p} 2}}{\longrightarrow} \mathrm{R}_{1}
\end{aligned}
$$

$\mathrm{R}_{1}+\mathrm{R}_{1} \stackrel{k_{\mathrm{t}}^{1,1}}{\longrightarrow}$ polymer

$\mathrm{R}_{1}+\mathrm{R}_{2} \stackrel{k_{\mathrm{t}}^{1,2}}{\longrightarrow}$ polymer

$\mathrm{R}_{2}+\mathrm{R}_{2} \stackrel{k_{\mathrm{t}}^{2,2}}{\longrightarrow}$ polymer

where $R_{1}$ and $R_{2}$ are secondary and tertiary radicals, respectively, and $M$ is monomer. The following two conditions are assumed to be fulfilled:

1. $k_{\mathrm{p} 2}<<k_{\mathrm{p} 1}$, that is, tertiary radicals are assumed to be propagating at a much slower rate than the secondary ones.

2. $\mathrm{R}_{2}+\mathrm{M} \stackrel{k_{\mathrm{p} 2}}{\longrightarrow} \mathrm{R}_{1}$, that is, after the first monomer addition step tertiary radicals "turn into" secondary ones.

A first assumption is to suppose that the different termination reactions occur with the same rate coefficient: $k_{\mathrm{t}}^{1,1}=k_{\mathrm{t}}^{1,2}=k_{\mathrm{t}}^{2,2}=k_{\mathrm{t}}$. This assumption gives:

$$
\begin{aligned}
-\frac{\mathrm{d} c_{\mathrm{M}}}{\mathrm{d} t} & =k_{\mathrm{p} 1} \cdot c_{\mathrm{M}} \cdot c_{\mathrm{R}_{1}}+k_{\mathrm{p} 2} \cdot c_{\mathrm{M}} \cdot c_{\mathrm{R}_{2}} \\
\frac{\mathrm{d} c_{\mathrm{R}_{1}}}{\mathrm{~d} t} & =v_{\mathrm{in}}-k_{\mathrm{bb}} \cdot c_{\mathrm{R}_{1}}+k_{\mathrm{p} 2} \cdot c_{\mathrm{M}} \cdot c_{\mathrm{R}_{2}}-k_{\mathrm{t}} \cdot c_{\mathrm{R}_{1}} \cdot c_{\mathrm{R}}
\end{aligned}
$$




$$
\frac{\mathrm{d} c_{\mathrm{R}_{2}}}{\mathrm{~d} t}=k_{\mathrm{bb}} \cdot c_{\mathrm{R}_{1}}-k_{\mathrm{p} 2} \cdot c_{\mathrm{M}} \cdot c_{\mathrm{R}_{2}}-k_{\mathrm{t}} \cdot c_{\mathrm{R}_{2}} \cdot c_{\mathrm{R}}
$$

where $c_{\mathrm{R}}=c_{\mathrm{R}_{1}}+c_{\mathrm{R}_{2}}$ is the overall radical concentration $\left(c_{\mathrm{R}}=\sqrt{\frac{\nu_{\mathrm{in}}}{k_{\mathrm{t}}}}\right)$. Assuming steady-state conditions, that is,

$$
\frac{\mathrm{d} c_{\mathrm{R}_{1}}}{\mathrm{~d} t}=\frac{\mathrm{d} c_{\mathrm{R}_{2}}}{\mathrm{~d} t} \approx 0
$$

one obtains from Eqs. 9.3 and 9.4:

$$
c_{\mathrm{R}_{2}}=\frac{k_{\mathrm{bb}}}{k_{\mathrm{bb}}+k_{\mathrm{p} 2} \cdot c_{\mathrm{M}}+\sqrt{k_{\mathrm{t}} \cdot v_{\mathrm{in}}}} \cdot c_{\mathrm{R}}
$$

Estimates for $k_{\mathrm{bb}}$ suggest that this value is of the order of $10^{2} \mathrm{~s}^{-1}$ at $60{ }^{\circ} \mathrm{C}$ [14]. For $k_{\mathrm{p} 2}$ a value of $50 \mathrm{~L} \cdot \mathrm{mol}^{-1} \cdot \mathrm{s}^{-1}[22]$ is estimated, the expression $\sqrt{k_{\mathrm{t}} \cdot v_{\text {in }}} \approx 1$ taking into account that typical generation rates $\left(\nu_{\text {in }}\right)$ are of order of $10^{-7} \mathrm{~mol} \cdot \mathrm{L}^{-1} \cdot \mathrm{s}^{-1}$ and $k_{\mathrm{t}}<10^{8} \mathrm{~L} \cdot \mathrm{mol}^{-1} \cdot \mathrm{s}^{-1}$ for BA homopolymerization [28]. Thus, Eq. 9.6 can be simplified assuming that $k_{\mathrm{bb}} \gg \sqrt{k_{\mathrm{t}} \cdot v_{\mathrm{in}}}$ and $k_{\mathrm{p} 2} \cdot c_{\mathrm{M}} \gg>\sqrt{k_{\mathrm{t}} \cdot v_{\text {in }}}$ :

$$
\frac{c_{\mathrm{R}_{2}}}{c_{\mathrm{R}}}=\frac{k_{\mathrm{bb}}}{k_{\mathrm{bb}}+k_{\mathrm{p} 2} \cdot c_{\mathrm{M}}}
$$

The same expression was reported by Nikitin and Hutchinson [15]. They considered the rate of termination of mid-chain radicals to be below the termination rate of secondary radicals of acrylates. This finding shows that Eq. 9.6a is independent of the rate of termination and can be used for estimates of $k_{\mathrm{bb}}$.

The rate of polymerization, defined as the first derivative of monomer concentration with respect to time from Eqs. 9.2, 9.6, is given by:

$$
-\frac{\mathrm{d} c_{\mathrm{M}}}{\mathrm{d} t} \approx k_{\mathrm{p} 1} \cdot c_{\mathrm{M}} \cdot \frac{c_{\mathrm{R}}}{k_{\mathrm{bb}}+k_{\mathrm{p} 2} \cdot c_{\mathrm{M}}+k_{\mathrm{t}} \cdot c_{\mathrm{R}}}\left(k_{\mathrm{p} 2} \cdot c_{\mathrm{M}}+k_{\mathrm{t}} \cdot c_{\mathrm{R}}+\frac{k_{\mathrm{p} 2}}{k_{\mathrm{p} 1}} \cdot k_{\mathrm{bb}}\right)
$$

Two limiting cases may be considered:

I. $k_{\mathrm{bb}} \ll k_{\mathrm{p} 2} \cdot c_{\mathrm{M}}$. The rate of intramolecular transfer to polymer is slow compared to the rate of propagation of tertiary radicals (reappearance of secondary radicals). The concentration of tertiary radicals in the system is negligible, $c_{\mathrm{R}_{2}}<<c_{\mathrm{R}_{1}}$, and the kinetics is close to ideal: 


$$
-\frac{\mathrm{d} c_{\mathrm{M}}}{\mathrm{d} t} \approx k_{\mathrm{p} 1} \cdot c_{\mathrm{M}} \cdot \sqrt{\frac{\nu_{\mathrm{in}}}{k_{\mathrm{t}}}}
$$

II. $k_{\mathrm{bb}} \gg k_{\mathrm{p} 2} \cdot c_{\mathrm{M}}$. The rate of propagation of tertiary radicals is slow compared to the rate of their production. Thus they are accumulating: $c_{\mathrm{R}} \approx c_{\mathrm{R}_{2}} \gg c_{\mathrm{R}_{1}}$. There are two possibilities:

a) "Slow" termination

If $k_{\mathrm{p} 2} \cdot c_{\mathrm{M}} \gg k_{\mathrm{t}} \cdot c_{\mathrm{R}}$, then

$$
\begin{gathered}
-\frac{\mathrm{d} c_{\mathrm{M}}}{\mathrm{d} t} \approx k_{\mathrm{p} 1} \cdot c_{\mathrm{M}} \frac{k_{\mathrm{p} 2} \cdot c_{\mathrm{M}}}{k_{\mathrm{bb}}} \cdot \sqrt{\frac{\nu_{\mathrm{in}}}{k_{\mathrm{t}}}} \\
-\frac{\mathrm{d} c_{\mathrm{M}}}{\mathrm{d} t} \propto c_{\mathrm{M}}^{2} \cdot c_{\mathrm{I}}^{0.5}
\end{gathered}
$$

b) "Fast" termination

In this case $k_{\mathrm{p} 2} \cdot c_{\mathrm{M}}<k_{\mathrm{t}} \cdot c_{\mathrm{R}}$ one obtains:

$$
-\frac{\mathrm{d} c_{\mathrm{M}}}{\mathrm{d} t} \approx k_{\mathrm{p} 1} \cdot c_{\mathrm{M}} \frac{v_{\mathrm{in}}}{k_{\mathrm{bb}}+\sqrt{v_{\mathrm{in}} \cdot k_{\mathrm{t}}}}
$$

If intramolecular transfer to polymer is the main chain-stopping event, then

$$
-\frac{\mathrm{d} c_{\mathrm{M}}}{\mathrm{d} t} \propto c_{\mathrm{M}}^{1} \cdot c_{\mathrm{I}}^{1}
$$

To summarize, if the rate of propagation of tertiary radicals is faster than the rate of intramolecular transfer to polymer, than the kinetics is governed by secondary radicals (their termination and propagation), even though the polymer chains will be branched. If, on the other hand, the rate of intramolecular transfer exceeds the rate of propagation of tertiary radicals, then the kinetics is governed by reactions between tertiary radicals. In general, the coefficient of intramolecular transfer to polymer (backbiting) should depend on both radical chain length and size and structure of the ester chain. The probability of such transfer (hydrogen abstraction) is higher for macroradicals and for monomers with longer ester groups, such as dodecyl acrylate. For the present estimates it was assumed that the rate coefficients are constant and independent of radicals size. 


\subsection{EXPERIMENTAL RESULTS}

In this section results from several polymerizations initiated by thermal decomposition of tertbutylperoxy-2-ethylhexanoate (TBPO) will be discussed. First, homopolymerizations of BA and DA will be presented. The model proposed in section 9.2 will be applied to the experimental results in order to describe polymerization kinetics of acrylates under steadystate conditions.

In section 6.3.1, termination kinetics of DMA at $0{ }^{\circ} \mathrm{C}$ was studied via the SP-PLP-ESR technique. In the following chapter the homopolymerization of DMA initiated by thermal decomposition of TBPO will be investigated. $k_{\mathrm{t}}$ values from chemically initiated polymerization will be compared with $k_{\mathrm{t}}$ values obtained by the SP-PLP-NIR and SP-PLPESR techniques.

\subsubsection{Chemically Initiated Polymerization of BA}

The Polymer Handbook [16] contains reports of propagation and termination rate coefficients for $n$-butyl acrylate. In general, researchers have carried out experiments using different temperatures and solvents at low conversion levels only. Reported values of rate coefficients for bulk, solution, or emulsion polymerizations can be found in Gladyshev et al. [17], Yokawa et al. , Kamachi et al. [18], Kaszas et al. [5], and Maxwell et al. [19], Beuermann et al. [28]. Full conversion range experiments were reported by Scott and Senogles [1,3] and Wunderlich [4]. The reaction order of BA obtained in these studies is presented in Tab. 9.1 in comparison with MA and DA and is equals 1.5.

For the systematic study into homopolymerization of BA all measurements in this work were carried out with the same initiator (TBPO) and in the same solvent (toluene).

Monomer conversions were determined as described in Section 4.7. Near infrared (NIR) spectra are recorded during the polymerization. These experiments show the decrease in the monomer concentration as a function of time. If one calculates the conversion from the monomer concentration one obtains a conversion-time curve.

Figs. 9.1, 9.2 and 9.3 show the conversion versus time plots for homopolymerizations of BA. In all cases conversion curves are continuous and do not show clear acceleration of the polymerization rate. 


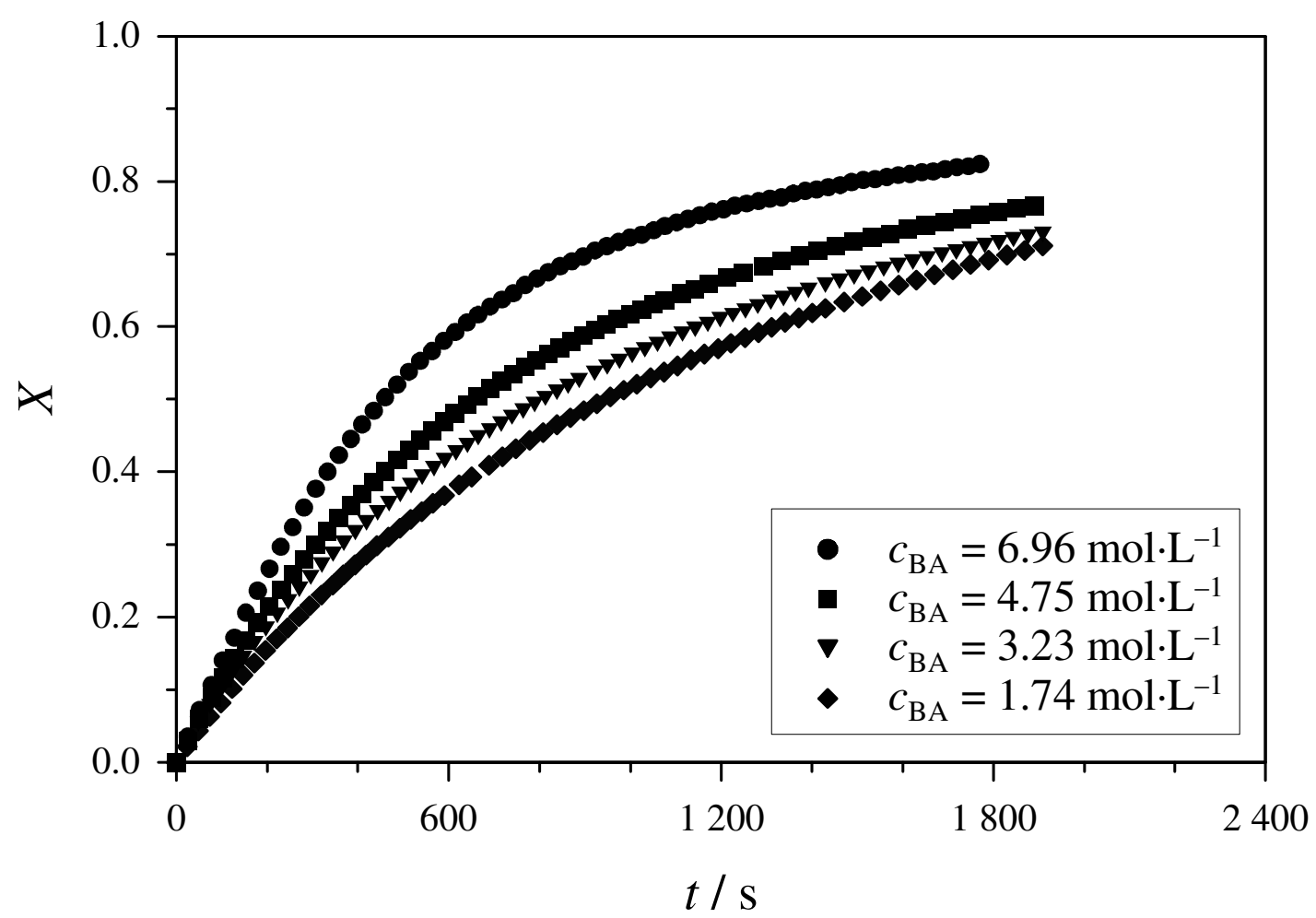

Fig. 9.1 Conversion vs. time plots for chemically initiated homopolymerizations of BA at different monomer concentrations in toluene solution, $c_{\mathrm{TBPO}}=5.8 \cdot 10^{-2} \mathrm{~mol} \cdot \mathrm{L}^{-1}, 65^{\circ} \mathrm{C}$.

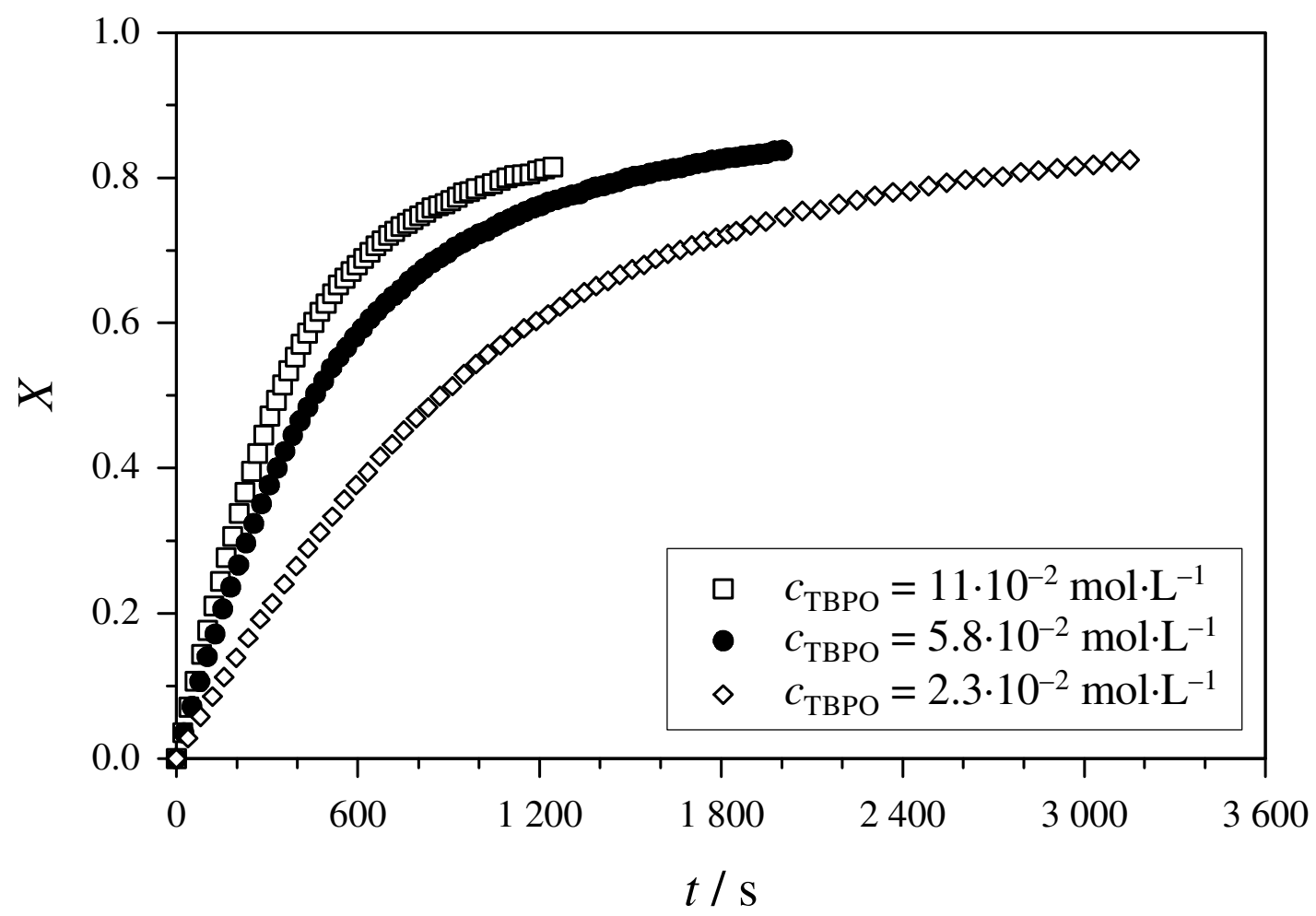

Fig. 9.2 Conversion vs. time plots for chemically initiated homopolymerizations of BA in bulk at different initiator concentrations, $65^{\circ} \mathrm{C}$. 


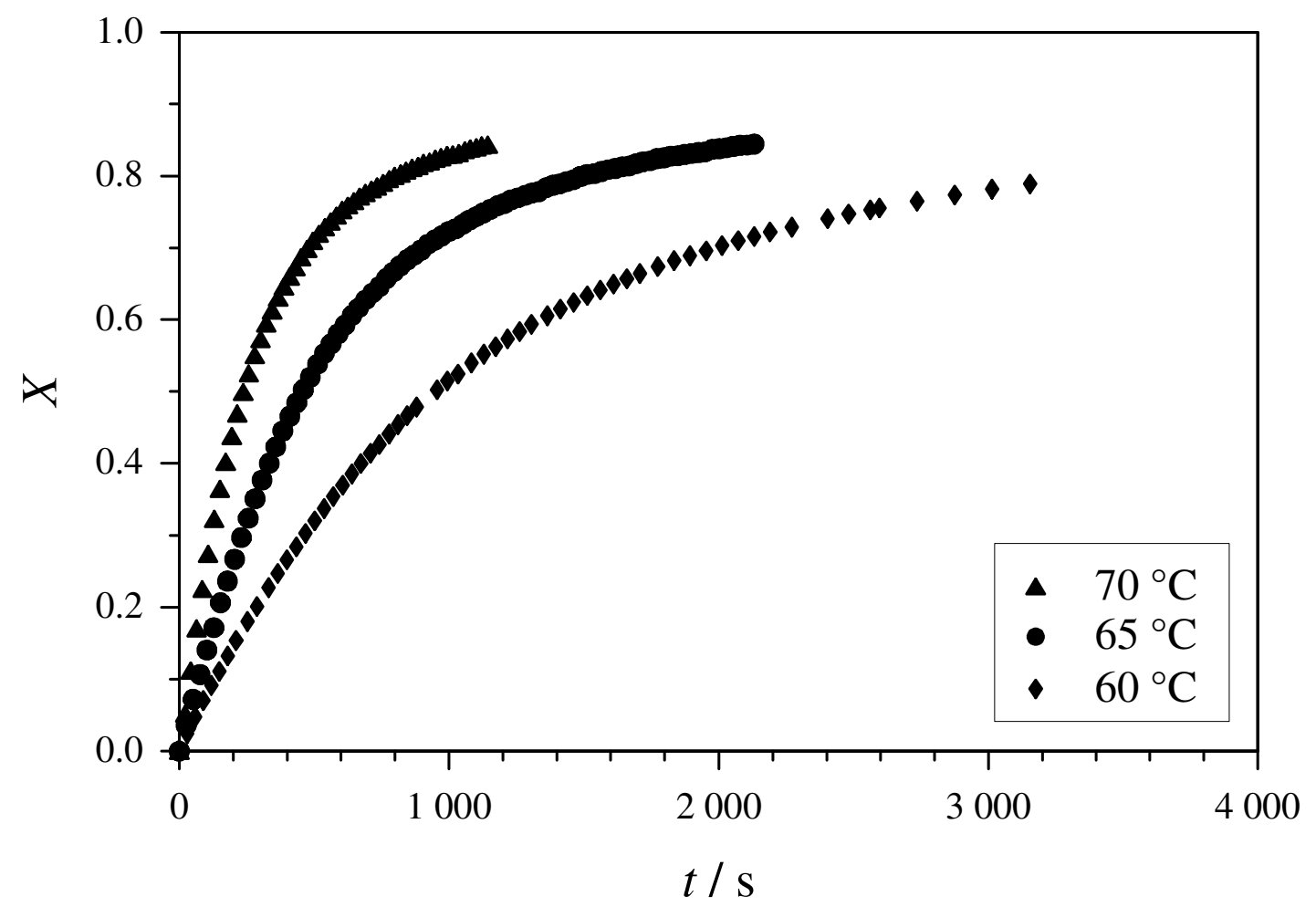

Fig. 9.3 Conversion vs. time plots for chemically initiated homopolymerizations of BA in bulk at different temperatures, $c_{\mathrm{TBPO}}=5.8 \cdot 10^{-2} \mathrm{~mol} \cdot \mathrm{L}^{-1}$.

In the chemically initiated polymerizations of BA the maximally attainable conversion was between 80 and 85 per cent of conversion. According to Horie et al. [20] the polymerization terminates at this conversion, because the polymer-monomer system passes over from a viscous fluid phase into a glasslike state. In this state neither translation diffusion of the monomer nor segmental diffusion of the chain ends of the polymer radicals is possible. By raising the temperature the transition to the glasslike phase is shifted toward higher conversions. Polymerizations at higher temperatures should therefore lead to greater final conversions. In acrylate polymerizations backbiting reactions lead to the production of midchain radicals (radicals with low propagation reactivity) that reduce the polymerization rate.

The linear kinetic curves (conversion as a function of time) may contain a "hidden" gel effect [1], which could be judged from the dependence of the overall polymerization rate on conversion. However, from the graphically illustrated dependences of the overall polymerization rate on conversion, it follows that the polymerization rate decreases continuously with increasing conversion from the start of polymerization for all monomer and initiator concentrations and all temperatures (Figs. 9.4, 9.5 and 9.6). 


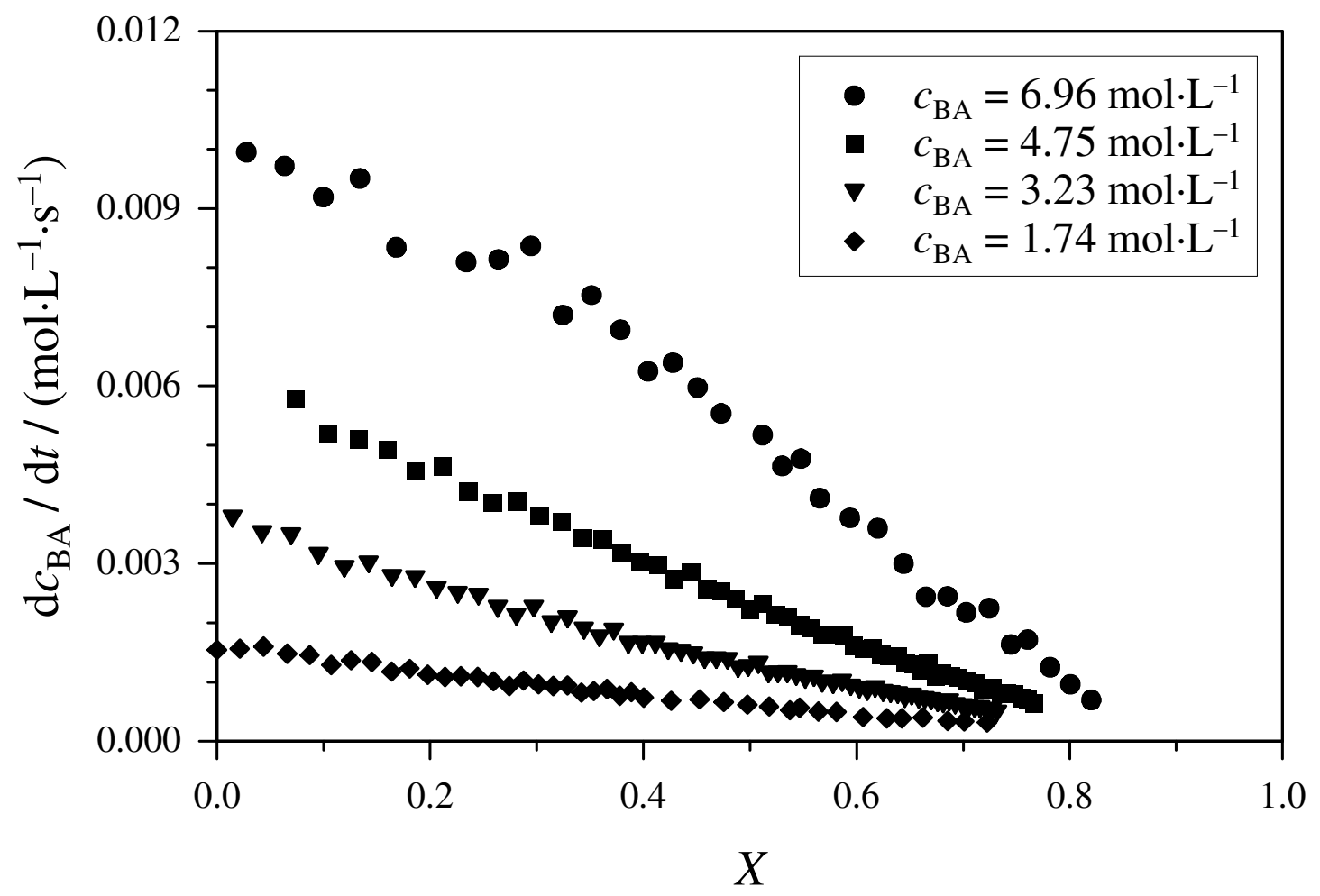

Fig. 9.4 Conversion dependence $(X)$ of overall reaction rate for chemically initiated homopolymerizations of $\mathrm{BA}$ at different monomer concentrations in toluene solution, $c_{\mathrm{TBPO}}=5.8 \cdot 10^{-2} \mathrm{~mol} \cdot \mathrm{L}^{-1}, 65^{\circ} \mathrm{C}$.

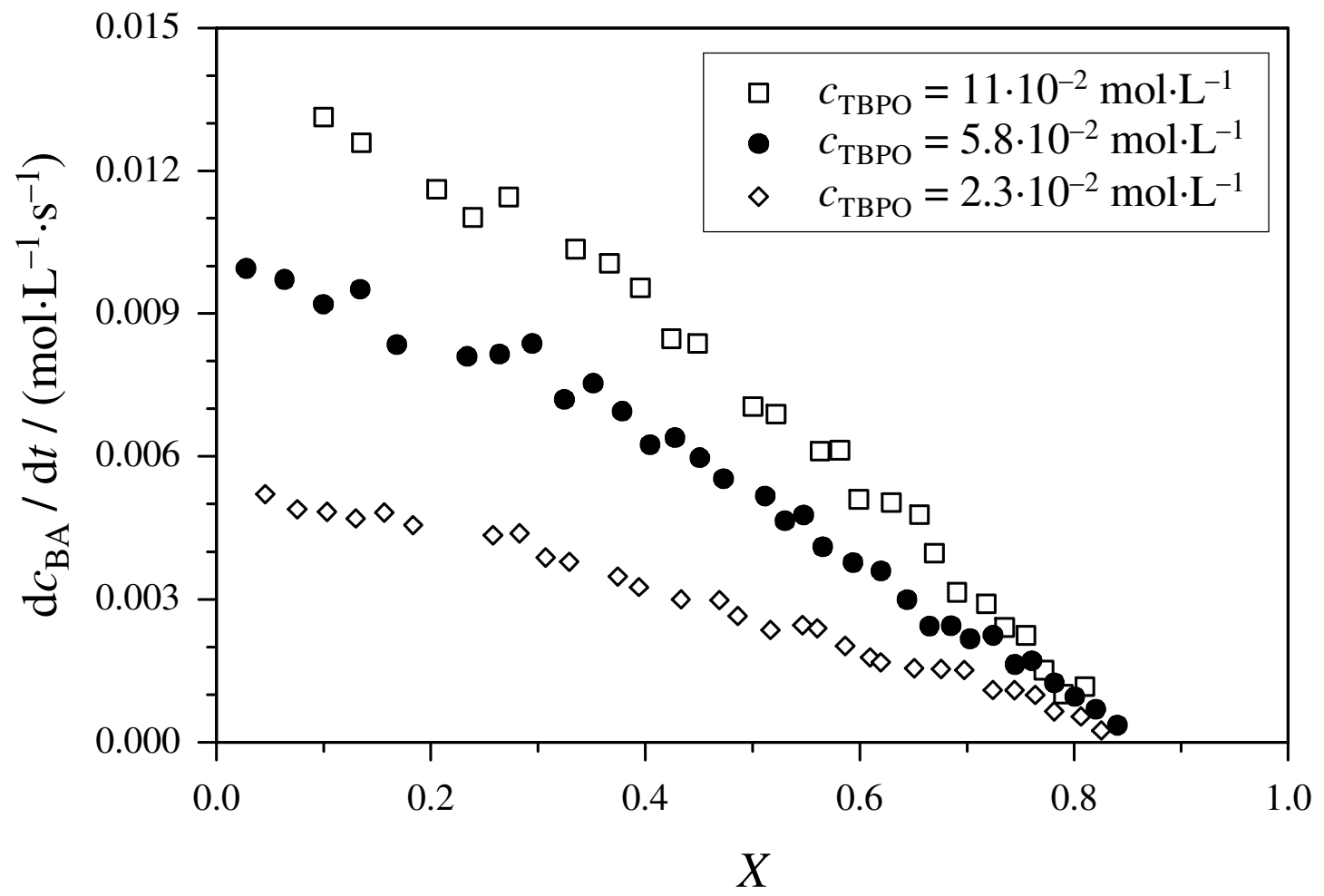

Fig. 9.5 Conversion dependence $(X)$ of overall reaction rate for chemically initiated homopolymerizations of BA in bulk at different initiator concentrations, $65{ }^{\circ} \mathrm{C}$. 


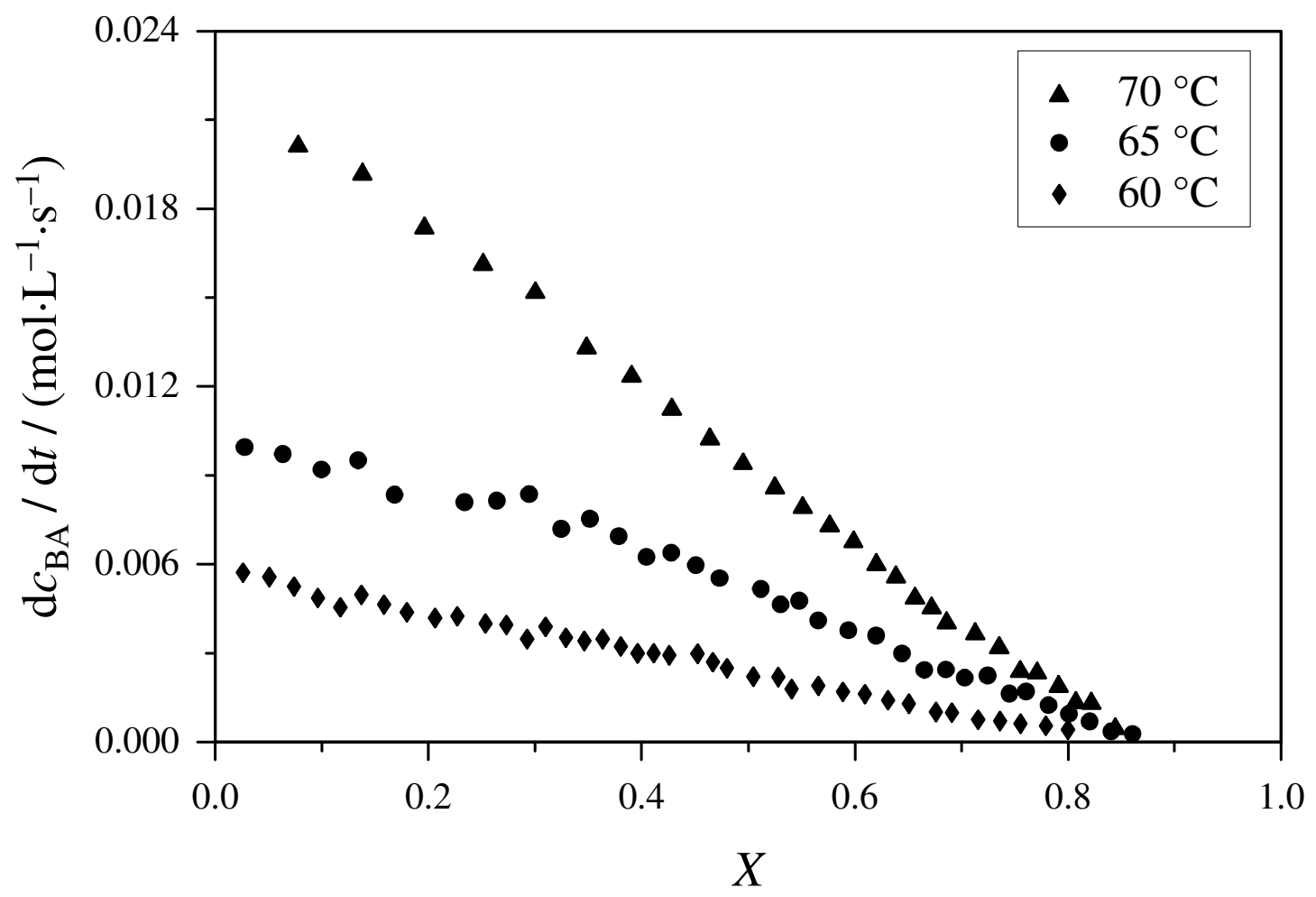

Fig. 9.6 Conversion dependence $(X)$ of overall reaction rate for chemically initiated homopolymerizations of $\mathrm{BA}$ in bulk at different temperatures, $c_{\mathrm{TBPO}}=5.8 \cdot 10^{-2} \mathrm{~mol} \cdot \mathrm{L}^{-1}$.

Fig. 9.4 shows that the initial concentration of monomer in solution has an strong influence on the rate of polymerization. As expected, the experiments with higher TBPO concentration are "faster" than the ones with lower initiator concentration (Fig. 9.5). With increasing temperature the rate of polymerization increases as well (Fig. 9.6).

The obtained results were used to estimate the coupled parameter $k_{\mathrm{p}} / k_{\mathrm{t}}^{0.5}$. However, it was necessary to choose a value for the initiator efficiency $f$ and the rate coefficient of initiator decomposition $k_{\mathrm{d}}$. A survey of the available literature revealed that very little reliable information on $f$ of TBPO is available for the BA systems. Therefore the values of $f$ determined by Sandmann in ethene polymerization and $k_{\mathrm{d}}$ determined in $n$-heptane [21] were used.

Tab. 9.2 contains values of $f$ and $k_{\mathrm{d}}$ for TBPO. The values of $k_{\mathrm{d}}$ are extrapolated to the present experimental conditions. 


\begin{tabular}{ccc}
\hline$\Theta /{ }^{\circ} \mathrm{C}$ & $k_{\mathrm{d}}(\mathrm{TBPO}) / \mathrm{s}^{-1}$ & $f_{\mathrm{TBPO}}$ \\
\hline 60 & $2.26 \cdot 10^{-6}$ & 0.64 \\
65 & $4.50 \cdot 10^{-6}$ & 0.64 \\
70 & $8.79 \cdot 10^{-6}$ & 0.64 \\
80 & $3.17 \cdot 10^{-5}$ & 0.64 \\
\hline
\end{tabular}

Tab. 9.2 The rate coefficient of initiator decomposition $\left(k_{\mathrm{d}}\right)$ and the initiator efficiency $(f)$ for TBPO as a function of temperature. $k_{\mathrm{d}}$ is recalculated to ambient pressure [21].

If the values of $f$ and $k_{\mathrm{d}}$ are known, measurements of conversion as a function of time allow to make an estimate of the coupled parameter $k_{\mathrm{p}} / k_{\mathrm{t}}^{0.5}$ from Eq. 9.1. Fig. 9.7 represents the correlation of the coupled parameter $k_{\mathrm{p}} / k_{\mathrm{t}}^{0.5}$ as a function of monomer concentration.

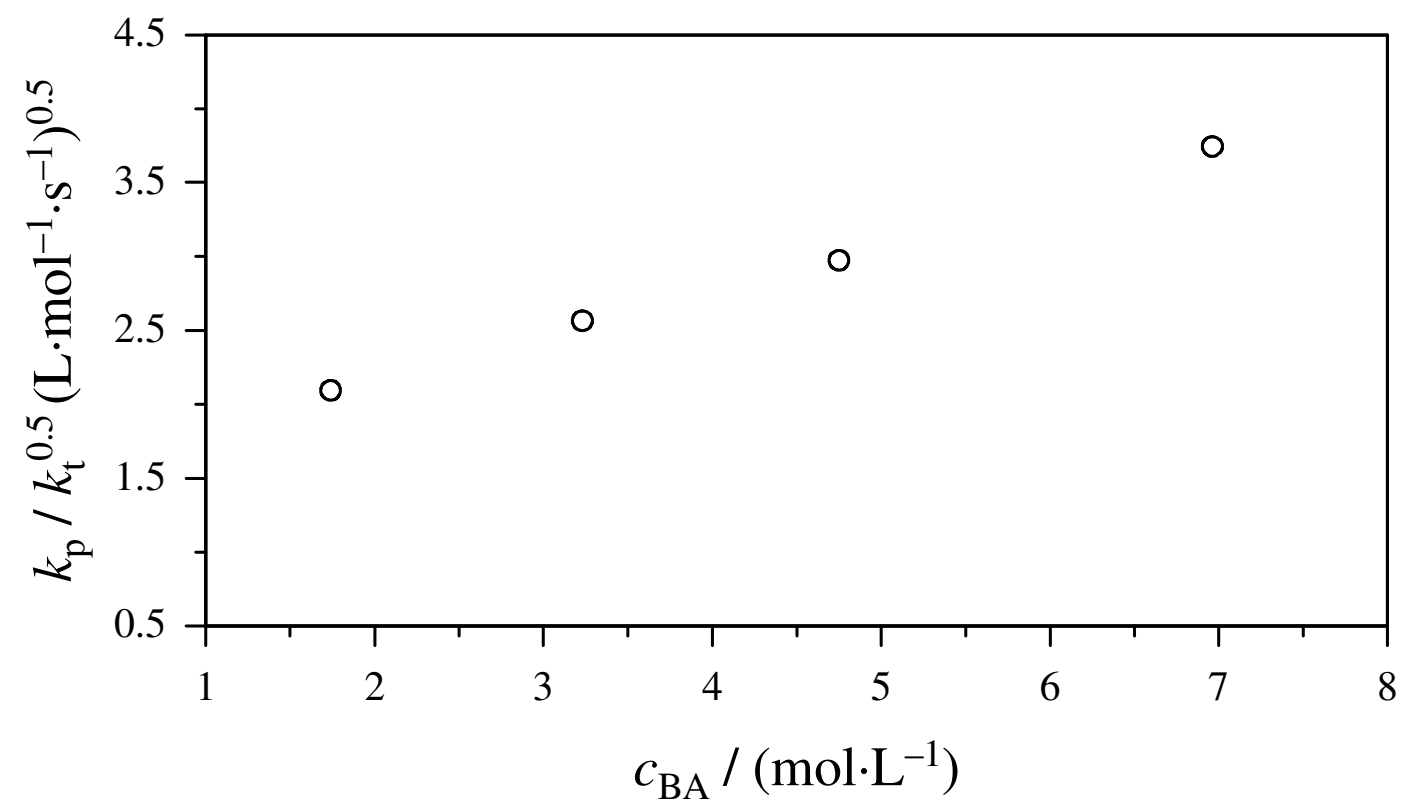

Fig. 9.7 Correlation of $k_{\mathrm{p}} / k_{\mathrm{t}}^{0.5}$ as a function of BA monomer concentration in toluene solution, $c_{\mathrm{TBPO}}=5.8 \cdot 10^{-2} \mathrm{~mol} \cdot \mathrm{L}^{-1}, 65^{\circ} \mathrm{C}$.

From Fig. 9.7 it is clear that the value of $k_{\mathrm{p}} / k_{\mathrm{t}}^{0.5}$ increases linearly as the initial monomer concentration in the solution of toluene increases. This is in good agreement with data reported in literature. It was shown by Madruga et al. [7] that $k_{\mathrm{p}} / k_{\mathrm{t}}^{0.5}$ for BA increased from 0.94 to 1.54 at $50{ }^{\circ} \mathrm{C}$ as the monomer concentration in benzene increased from 1 to $5 \mathrm{~mol} \cdot \mathrm{L}^{-1}$. Kaszas et al. [5] have shown that the increase of BA concentration from 0.305 to $6.79 \mathrm{~mol} \cdot \mathrm{L}^{-1}$ in benzene leads to almost a five-fold increase in $k_{\mathrm{p}} / k_{\mathrm{t}}^{0.5}$. 
Fig. 9.8 shows increase of the coupled parameter $k_{\mathrm{p}} / k_{\mathrm{t}}^{0.5}$ with temperature.

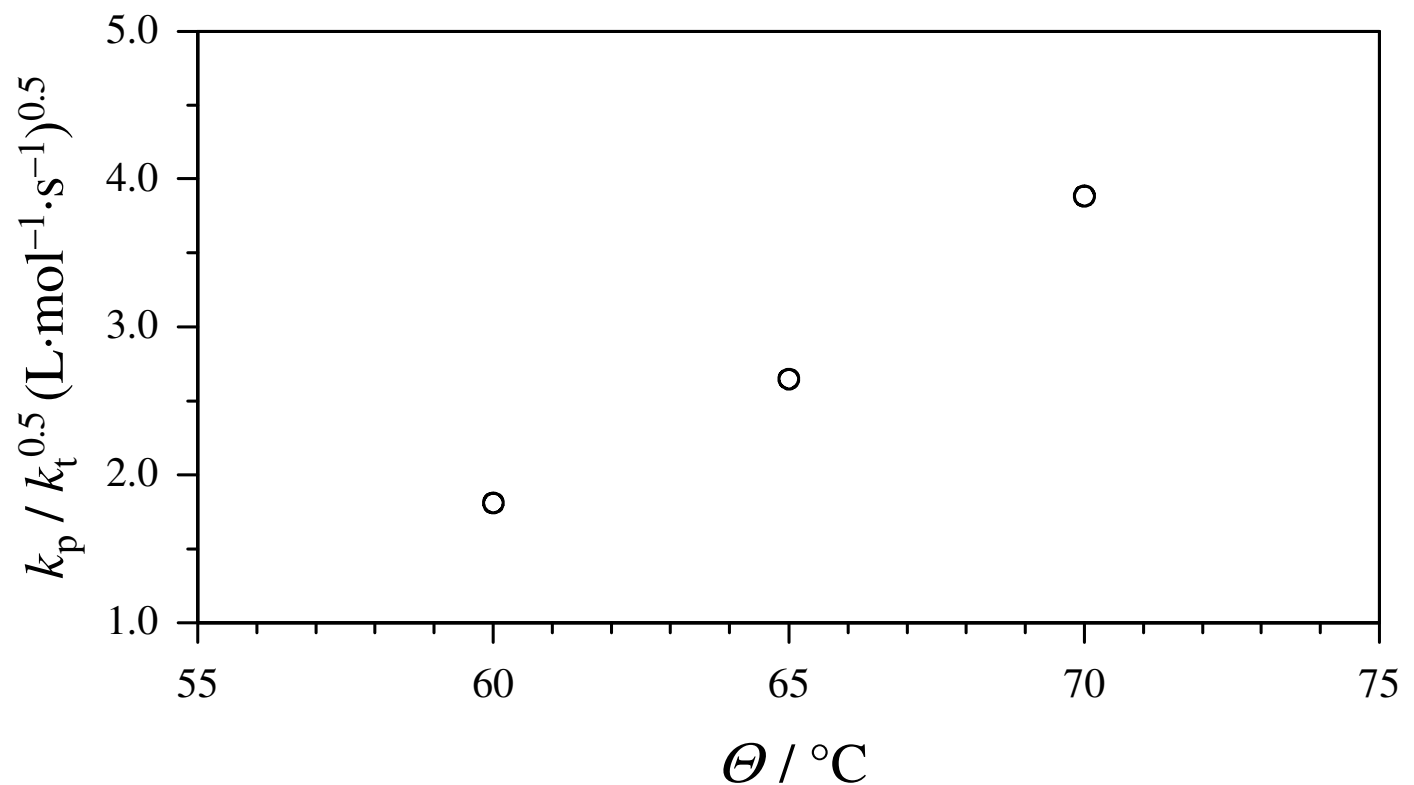

Fig. 9.8 Correlation of $k_{\mathrm{p}} / k_{\mathrm{t}}^{0.5}$ as a function of temperature, BA bulk polymerization, $c_{\mathrm{TBPO}}=5.8 \cdot 10^{-2} \mathrm{~mol} \cdot \mathrm{L}^{-1}$.

Various hypotheses to explain the increase of the coupled parameter $k_{\mathrm{p}} / k_{\mathrm{t}}^{0.5}$ with increasing monomer concentration in the system for acrylates have been proposed, including primary radical termination and degradative chain transfer [4], chain-length dependence of termination rate coefficients [9], the hot radical theory [5], and monomer/solvent complexation [3]. Among these explanations is also the influence of intramolecular transfer to polymer, proposed by Scott and Senogles [3].

Since the pioneering work of Scott and Senogles, strong evidence has emerged showing that backbiting and slow reinitiation of the resulting mid-chain radical are indeed important processes for acrylate polymerization, even at low temperatures.

Another strong indication for the deviation of acrylates from the ideal kinetic scheme would be the determination of the reaction order with respect to monomer. As it was already pointed out in section 9.1, the ideal kinetic schema for radical polymerization predicts a monomer reaction order of unity and an order 0.5 with respect to initiator concentration. For the determination of these two values the double logarithmic graph of the overall polymerization rate vs. monomer (or initiator) concentration should be plotted. From the slopes in Figs. 9.9 and 9.10 the exponent for the initiator reaction order is determined to be 0.62 and for BA in toluene as solvent at $65{ }^{\circ} \mathrm{C}$ is found to be 1.46 . The comparison was made at 30 per cent of conversion in all cases. 


$$
-\frac{\mathrm{d} c_{\mathrm{BA}}}{\mathrm{d} t} \propto c_{\mathrm{BA}}^{1.46} \cdot c_{\mathrm{TBPO}}^{0.62}
$$

The obtained values for BA are in good agreement with already reported data (Tab. 9.1).

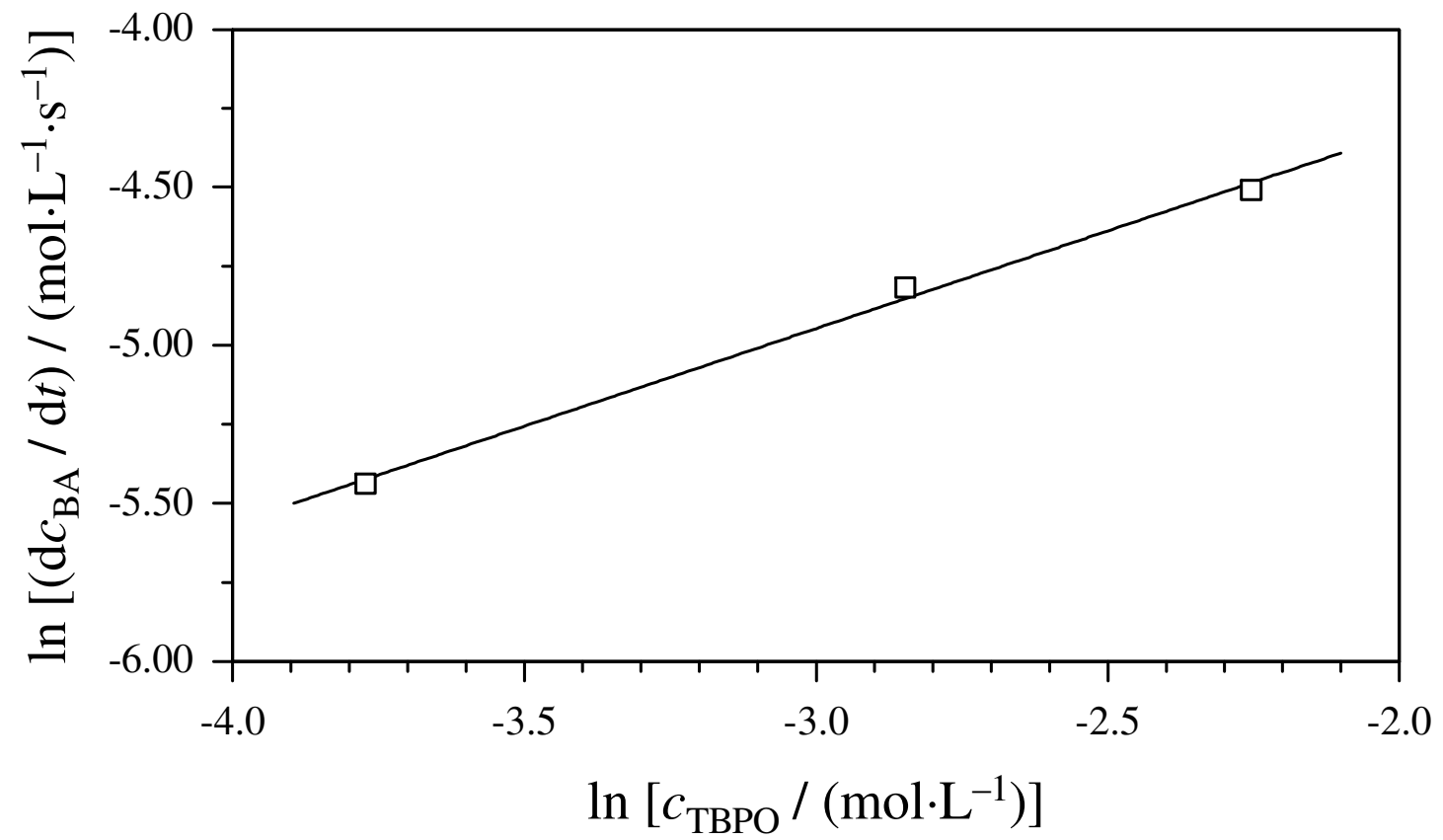

Fig. 9.9 Double logarithmic plot of overall reaction rate of BA in bulk vs. initiator concentration at 30 per cent of conversion, $65^{\circ} \mathrm{C}$.

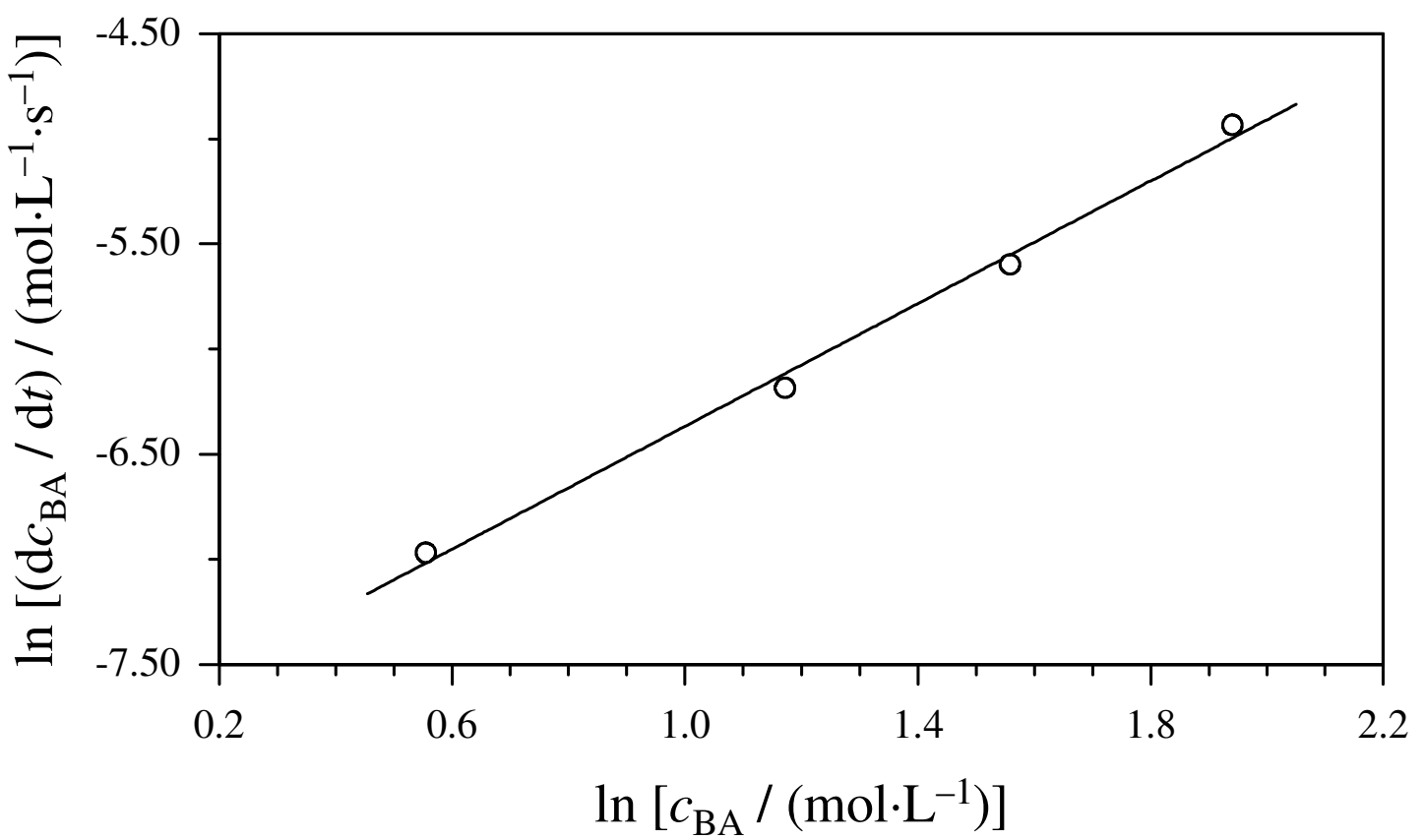

Fig. 9.10 Double logarithmic plot of the overall reaction rate of BA vs. monomer concentration in toluene at 30 per cent of conversion, $c_{\mathrm{TBPO}}=5.8 \cdot 10^{-2} \mathrm{~mol} \cdot \mathrm{L}^{-1}$, $65{ }^{\circ} \mathrm{C}$. 
Taking the model proposed in section 9.2 into account, one can see that the kinetics of BA homopolymerization is close to the case II.b. In this case the rate of propagation of tertiary radicals is much slower than the rate of production with an additional assumption $k_{\mathrm{p} 2} \cdot c_{\mathrm{M}} \gg k_{\mathrm{t}} \cdot c_{\mathrm{R}}$. Thus, the role of tertiary radicals in free-radical polymerization of BA cannot be neglected.

For the estimate of the intramolecular transfer coefficient, $k_{\mathrm{bb}}$, Eq. 9.6a can be used. The ratio of $c_{\mathrm{R}_{2}} / c_{\mathrm{R}}$ in Eq. 9.6a was obtained from laser-initiated ESR experiments presented in section 7.2.1 as well from ESR measurements of BA polymerization initiated by thermal decomposition of TBPO at $65^{\circ} \mathrm{C}$. $k_{\mathrm{p} 2}$ values were assumed to be close to the propagation rate coefficients of BAD [22]. In Fig. 9.11 an Arrhenius plot of calculated rate coefficients is given.

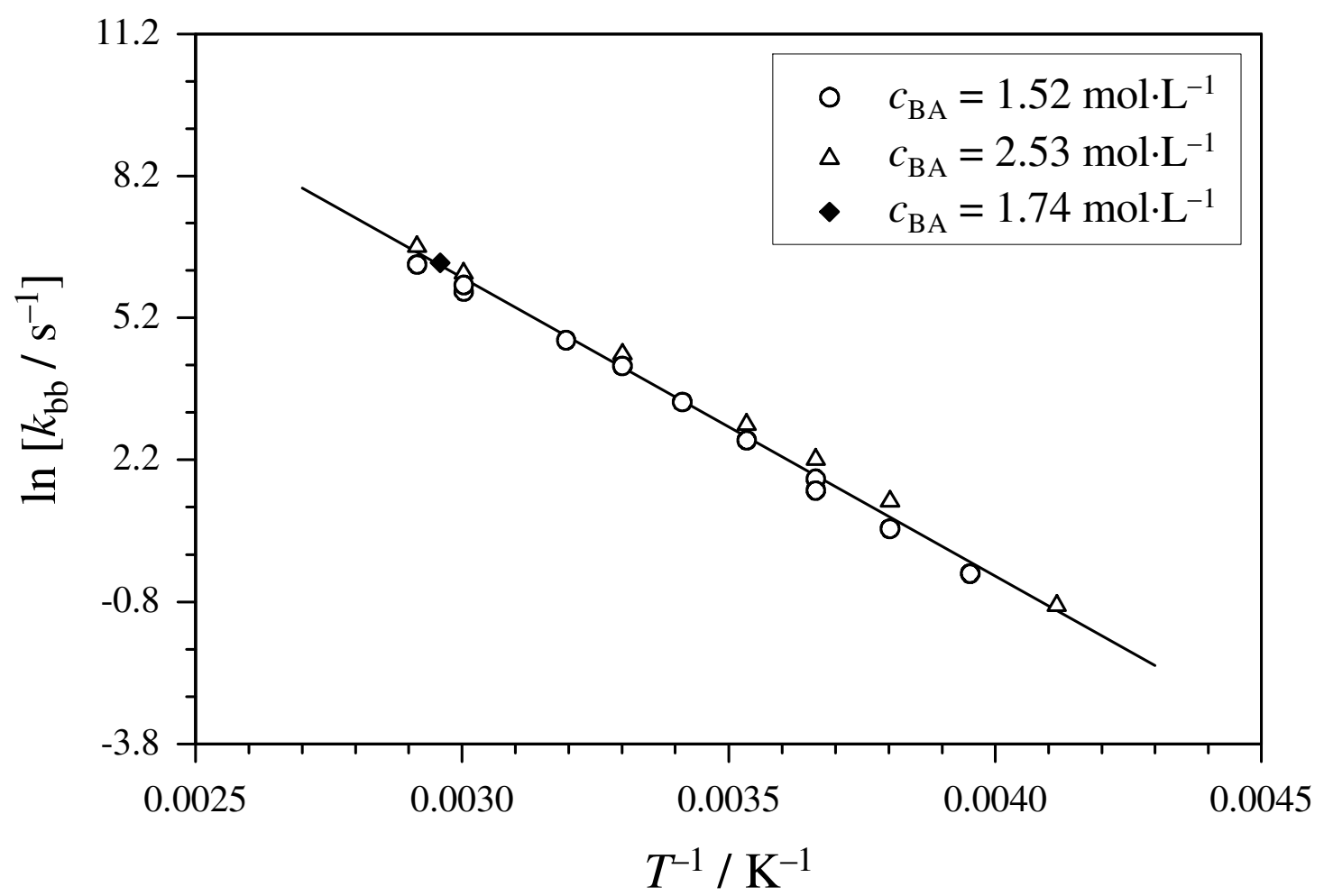

Fig. 9.11 Temperature dependence of the intramolecular transfer coefficient, $k_{\mathrm{bb}}$, obtained via Eq. 9.6a for BA free-radical homopolymerization at ambient pressure. The ratio of $c_{\mathrm{R}_{2}} / c_{\mathrm{R}}$ in Eq. 9.6a was obtained from laser initiated ESR experiments presented in section 7.2.1 (open symbols) and from ESR measurements of BA polymerization initiated by thermal decomposition of TBPO (full symbol). 
The Arrhenius expression from Fig. 9.11 for $k_{\mathrm{bb}}$ of BA is presented by Eq. 9.13:

$$
\ln \left[k_{\mathrm{bb}} / \mathrm{s}^{-1}\right]=24.94-6296 \cdot\left(T^{-1} / \mathrm{K}^{-1}\right)
$$

(BA homopolymerization at ambient pressure, $-40 \leq \Theta /{ }^{\circ} \mathrm{C} \leq 70$ ).

From the slope to the straight line in Fig. 9.11, the activation energy was estimated to be: $E_{\mathrm{A}}\left(k_{\mathrm{bb}}\right)=(52.4 \pm 9.1) \mathrm{kJ} \cdot \mathrm{mol}^{-1}$.

In the literature, the value of the activation energy for backbiting in BA free-radical polymerization was estimated via Monte Carlo simulations to be $29.8 \mathrm{~kJ} \cdot \mathrm{mol}^{-1}$ [14]. This value is lower than one obtained in this work. On the other hand, for ethylene homopolymerization an activation energy of $44.8 \mathrm{~kJ} \cdot \mathrm{mol}^{-1}$ for backbiting was reported [23].

\section{PREDICI Simulations}

Tab. 9.3 presents the frequency factors and activation energies of the rate coefficients used in the simulations for the two types of radicals. Values of $k_{\mathrm{p} 1}$ were estimated from the values obtained by PLP-SEC at lower temperatures [25,26], the values of termination rate coefficient $k_{\mathrm{t}}^{1,1}$ were extrapolated from SP-PLP-NIR experiments, performed at 1000 bar, to ambient pressure with an activation volume of $V_{40}^{\neq}{ }^{\circ} \mathrm{C}=15.0 \mathrm{~cm}^{3} \cdot \mathrm{mol}^{-1}$ [27]. The frequency factor and the activation energy for the propagation rate coefficient of the tertiary radicals in BA homopolymerization were taken from the results for the butyl acrylate dimer (BAD)[22]. BAD yields a tertiary radical similar to that generated after chain transfer to polymer in BA homopolymerization. There is no literature data for termination rate coefficients of mid-chain radicals produced in the intramolecular chain transfer reaction. In the present study it was difficult to describe conversion dependence of BA homopolymerization as a function of time for BA homopolymerization using only one value of termination rate coefficient $k_{\mathrm{t}}^{1,1}$. Thus, the assumption, made in Section 9.2 that the different termination reactions occur with the same rate coefficient is too strong. Hence, for mid-chain radicals values obtained for the family of itaconates (that also yields structurally hindered tertiary radicals) were taken [24]. It should be noted that the mobility of hindered itaconate radicals will be more reduced in comparison with the mid-chain radicals formed in acrylate homopolymerization for which most of the monomer units in a polymer chain will be flexible acrylate units. 


\begin{tabular}{cccc}
\hline BA & $A / \mathrm{L} \cdot \mathrm{mol}^{-1} \cdot \mathrm{s}^{-1}$ & $E_{\mathrm{A}} / \mathrm{kJ} \cdot \mathrm{mol}^{-1}$ & Source \\
\hline Secondary radical & $1.8 \cdot 10^{7}$ & & \\
Propagation, $k_{\mathrm{p} 1}$ & $2.9 \cdot 10^{8}$ & 17.4 & {$[25,26]$} \\
Termination, $k_{\mathrm{t}}^{1,1}$ & $6.8 \cdot 10^{10}$ & 4.0 & {$[27,28]$} \\
Backbiting, $k_{\mathrm{bb}}$ & & 52.3 & this work \\
Mid-chain radical & $9.2 \cdot 10^{6}$ & & \\
Propagation, $k_{\mathrm{p} 2}$ & $1.6 \cdot 10^{9}$ & 33.8 & {$[22]$} \\
Termination, $k_{\mathrm{t}}^{2,2}$ & & 23.0 & {$[24]$} \\
\hline
\end{tabular}

Tab. 9.3 The frequency factors and the activation energies of rate coefficients for PREDICI® simulations.

For PREDICI ${ }^{\circledR}$ simulations it was assumed that the rate coefficient for termination of radicals from the two different populations $\mathrm{R}_{1}$ and $\mathrm{R}_{2}$ can be approximated by the geometric mean of $k_{\mathrm{t}}^{1,1}$ and $k_{\mathrm{t}}^{2,2}$ and that the secondary and tertiary radicals both have the same mode of termination. With little data available for such radicals, the chain-length dependence of termination rate coefficients was not considered.

Fig. 9.12 presents the simulated via PREDICI ${ }^{\circledR}$ conversion dependence of the overall reaction rate in comparison with measured data.

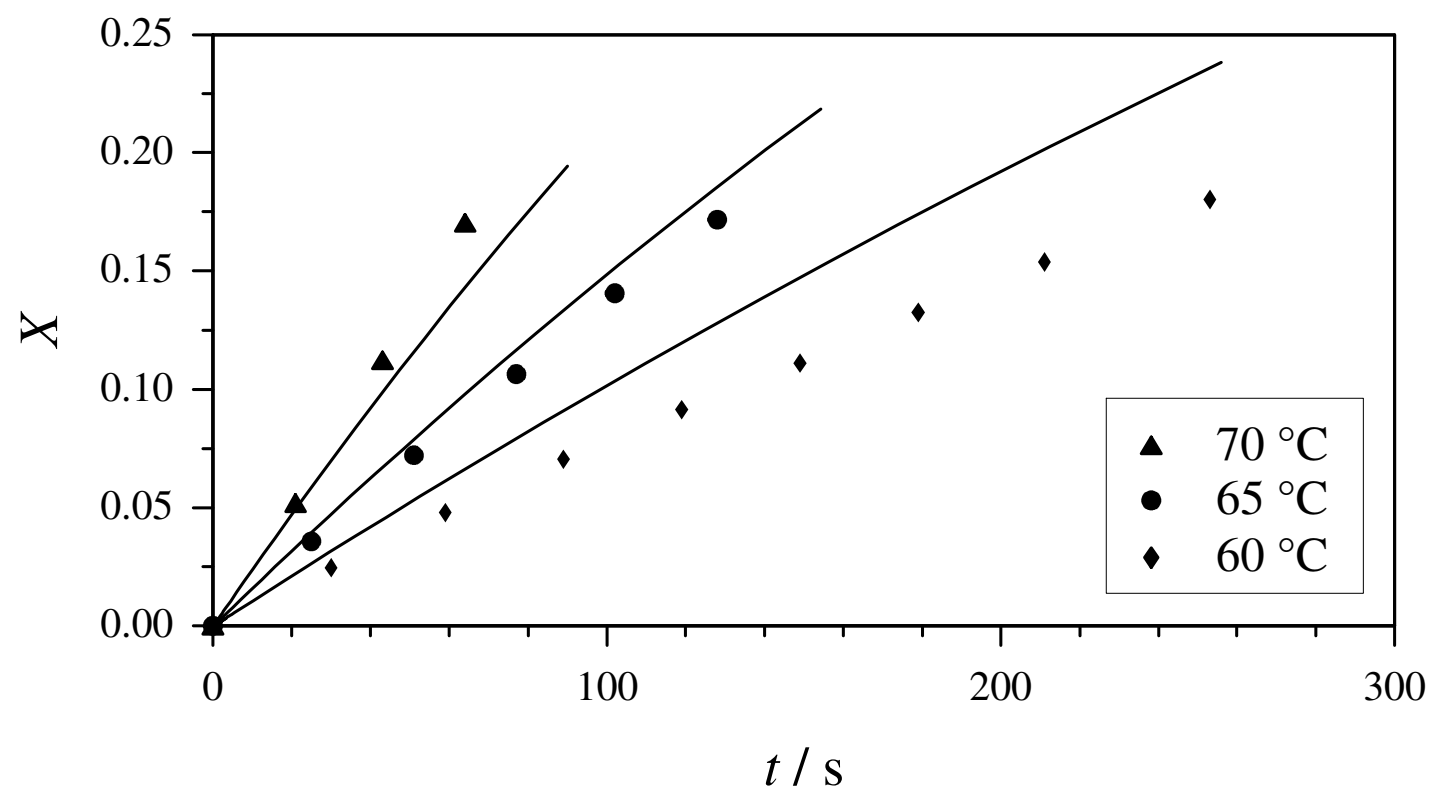

Fig. 9.12 Measured (points) and simulated (lines) conversion dependence $(X)$ of the overall reaction rate for chemically initiated bulk homopolymerization of BA at different temperatures, $c_{\mathrm{TBPO}}=5.8 \cdot 10^{-2} \mathrm{~mol} \cdot \mathrm{L}^{-1}$. 
As it can be seen in Fig. 9.12, the proposed kinetic scheme describes the bulk homopolymerization of BA reasonably well, in particular at $65^{\circ} \mathrm{C}$, with rate coefficients taken from Tab. 9.3 up to 10 per cent of conversion.

\subsubsection{Chemically INitiated Polymerization of DA}

The homopolymerization of DA was investigated by Scott and Senogles [1,2,3].

In order to obtain systematic data for the homopolymerization of DA, all measurements in this work were carried out with the same initiator (TBPO) and in the same solvent (toluene).

Figs. 9.13, 9.14 and 9.15 present the conversion versus time plots for various homopolymerizations of DA. In all cases conversion curves are continuous and do not show acceleration of the polymerization rate. The same observation was made by Scott and Senogles [1].

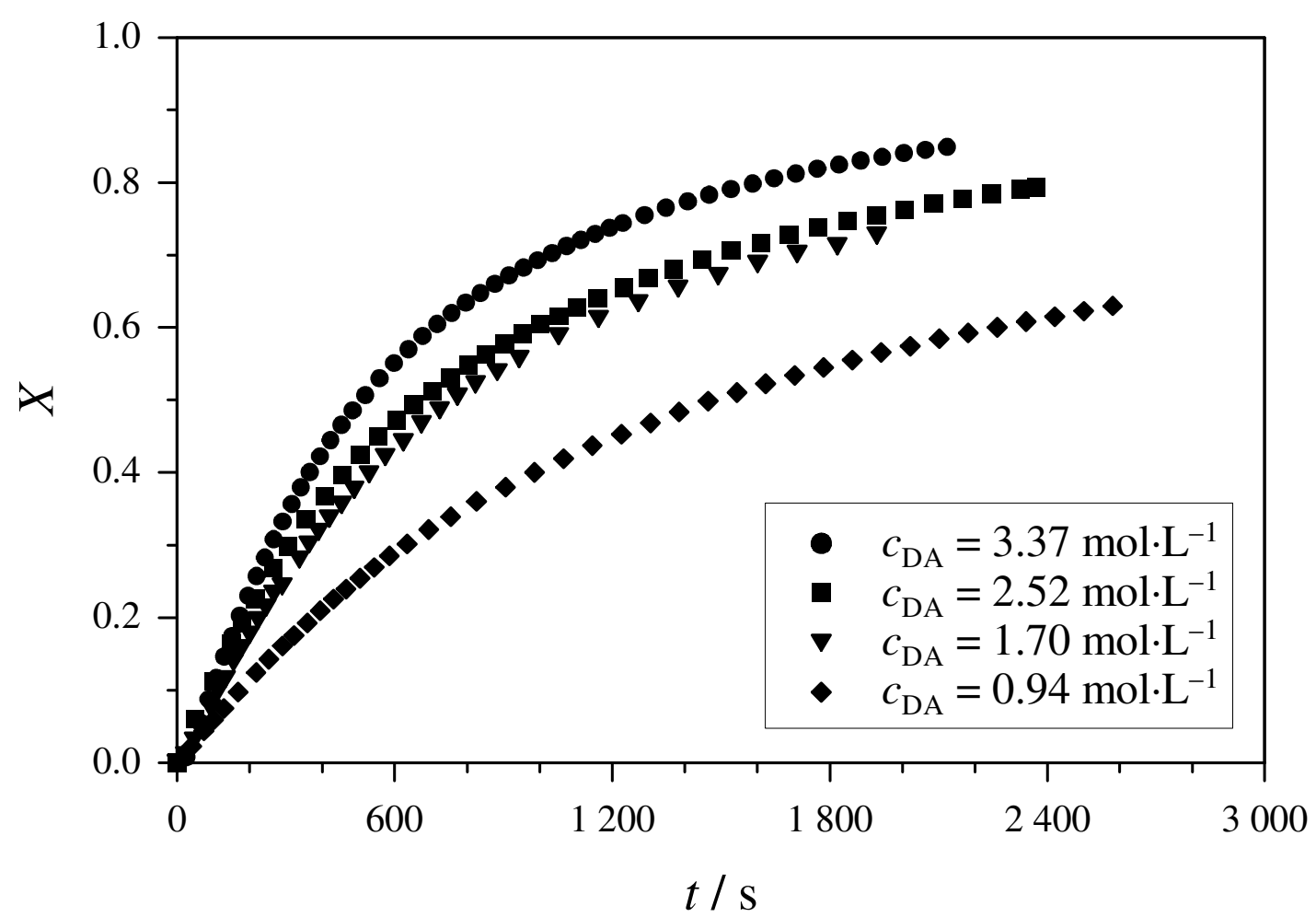

Fig. 9.13 Conversion vs. time plots for chemically initiated homopolymerizations of DA at different monomer concentrations in toluene solution, $c_{\mathrm{TBPO}}=8.4 \cdot 10^{-2} \mathrm{~mol} \cdot \mathrm{L}^{-1}, 60{ }^{\circ} \mathrm{C}$. 


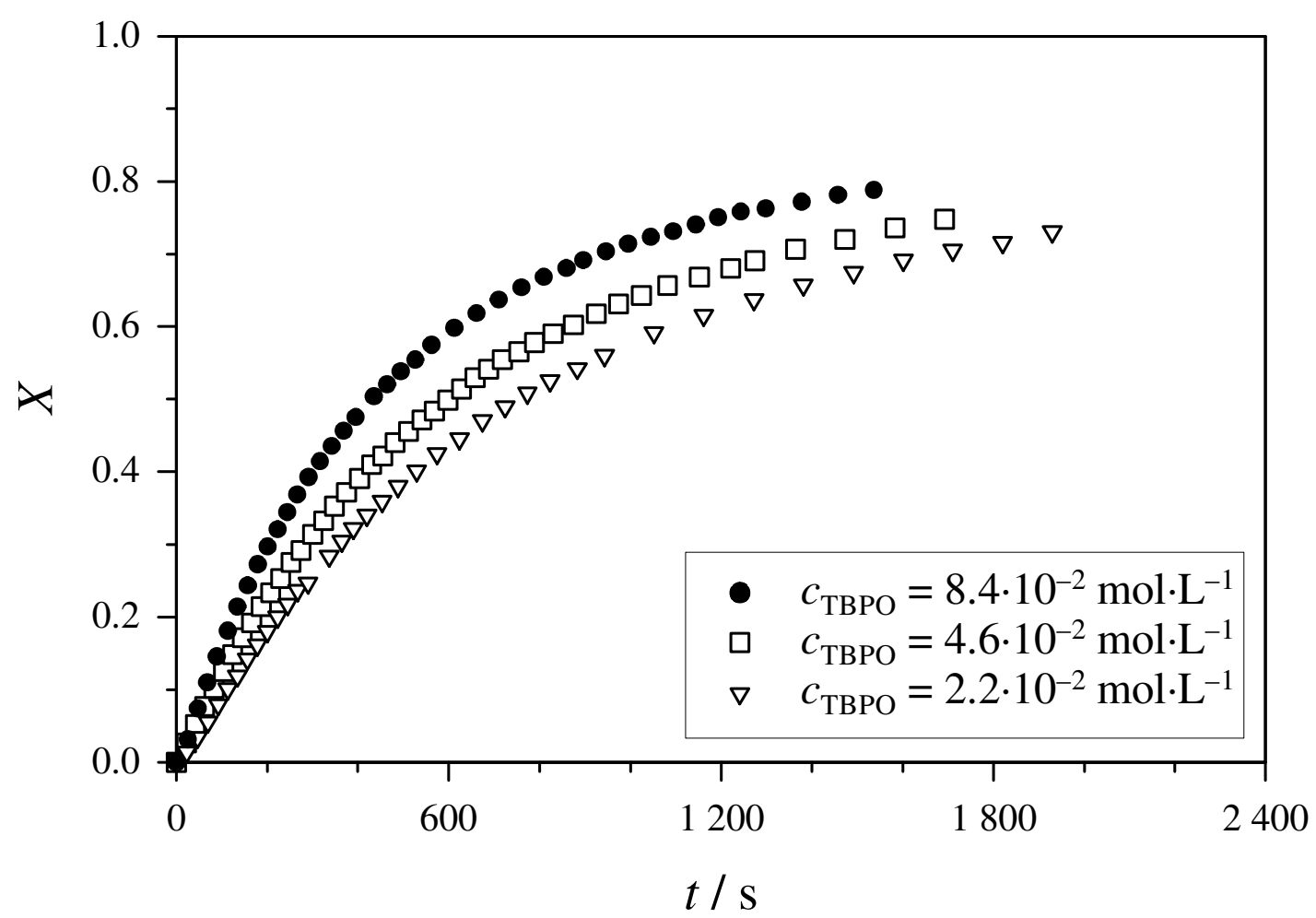

Fig. 9.14 Conversion vs. time plots for chemically initiated homopolymerizations of DA in bulk at different initiator concentrations, $60^{\circ} \mathrm{C}$.

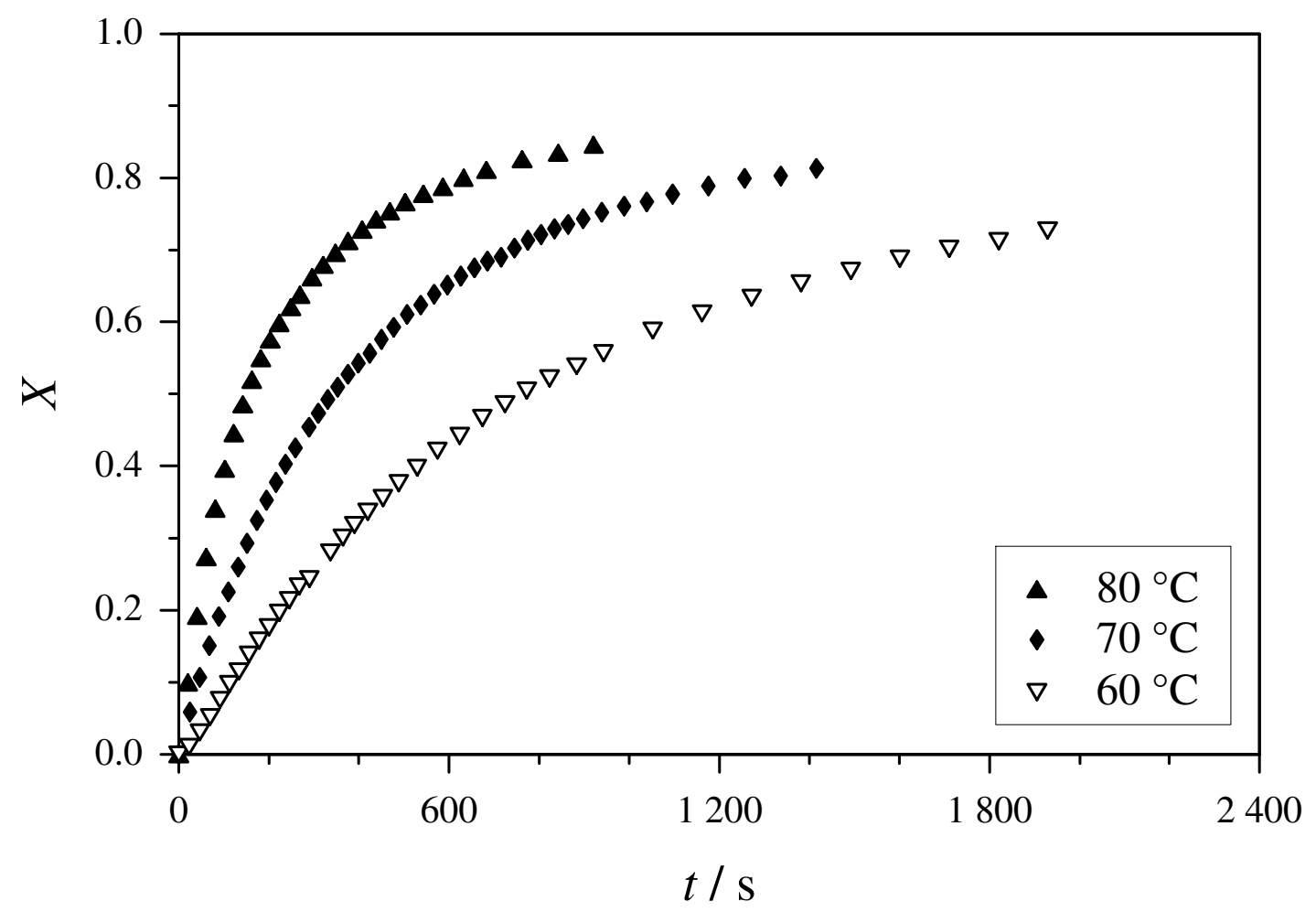

Fig. 9.15 Conversion vs. time plots for chemically initiated homopolymerizations of DA in bulk at different temperatures, $c_{\mathrm{TBPO}}=2.2 \cdot 10^{-2} \mathrm{~mol} \cdot \mathrm{L}^{-1}$. 
From the graphically illustrated dependences of the overall polymerization rate on conversion, it follows that the polymerization rate decreases continuously with increasing conversion from the start of polymerization for all monomer and initiator concentrations and all temperatures (Figs. 9.16, 9.17 and 9.18).

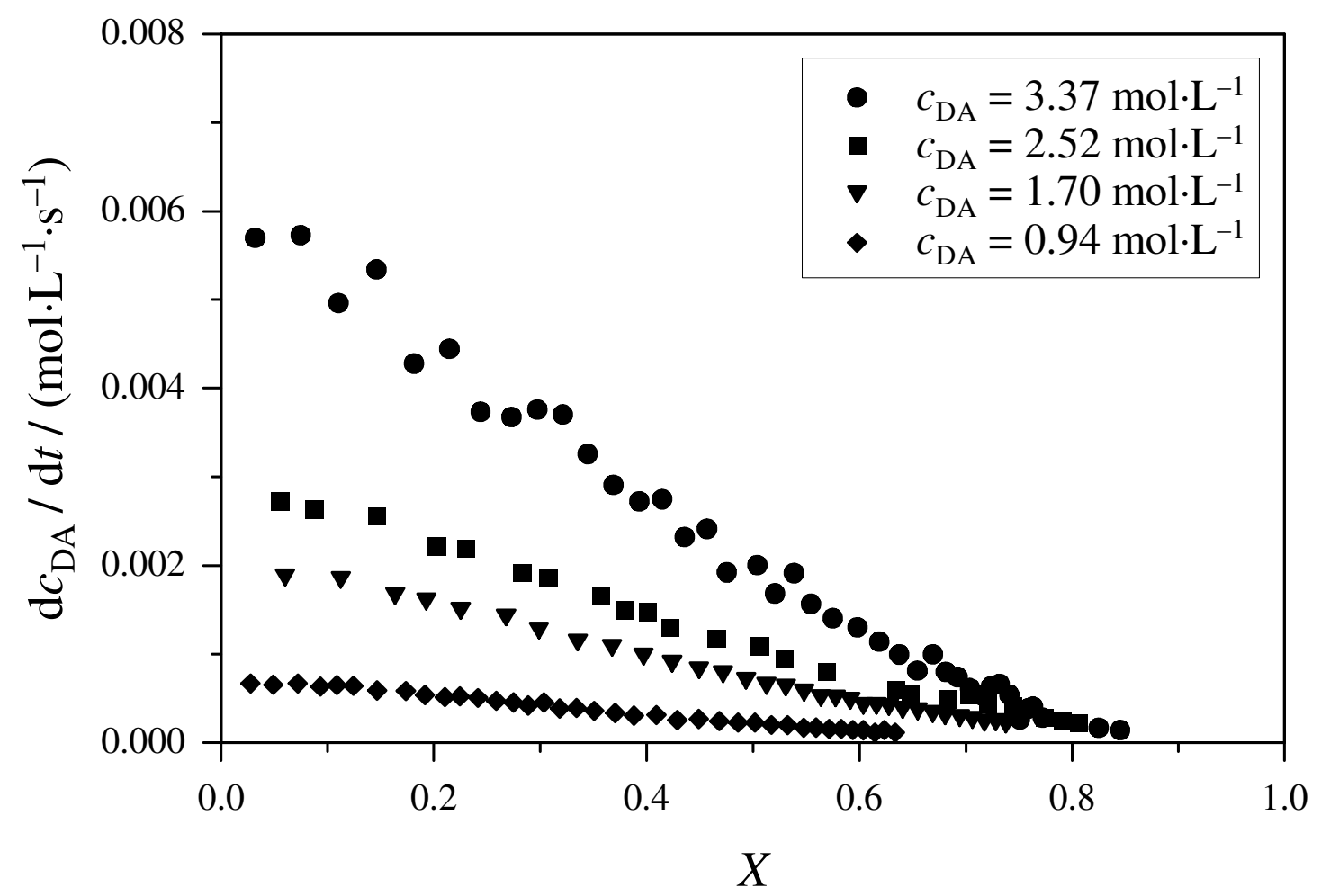

Fig. 9.16 Conversion dependence $(X)$ of overall reaction rate for chemically initiated homopolymerization of DA at different monomer concentrations in toluene solution, $c_{\mathrm{TBPO}}=8.4 \cdot 10^{-2} \mathrm{~mol} \cdot \mathrm{L}^{-1}, 60{ }^{\circ} \mathrm{C}$. 


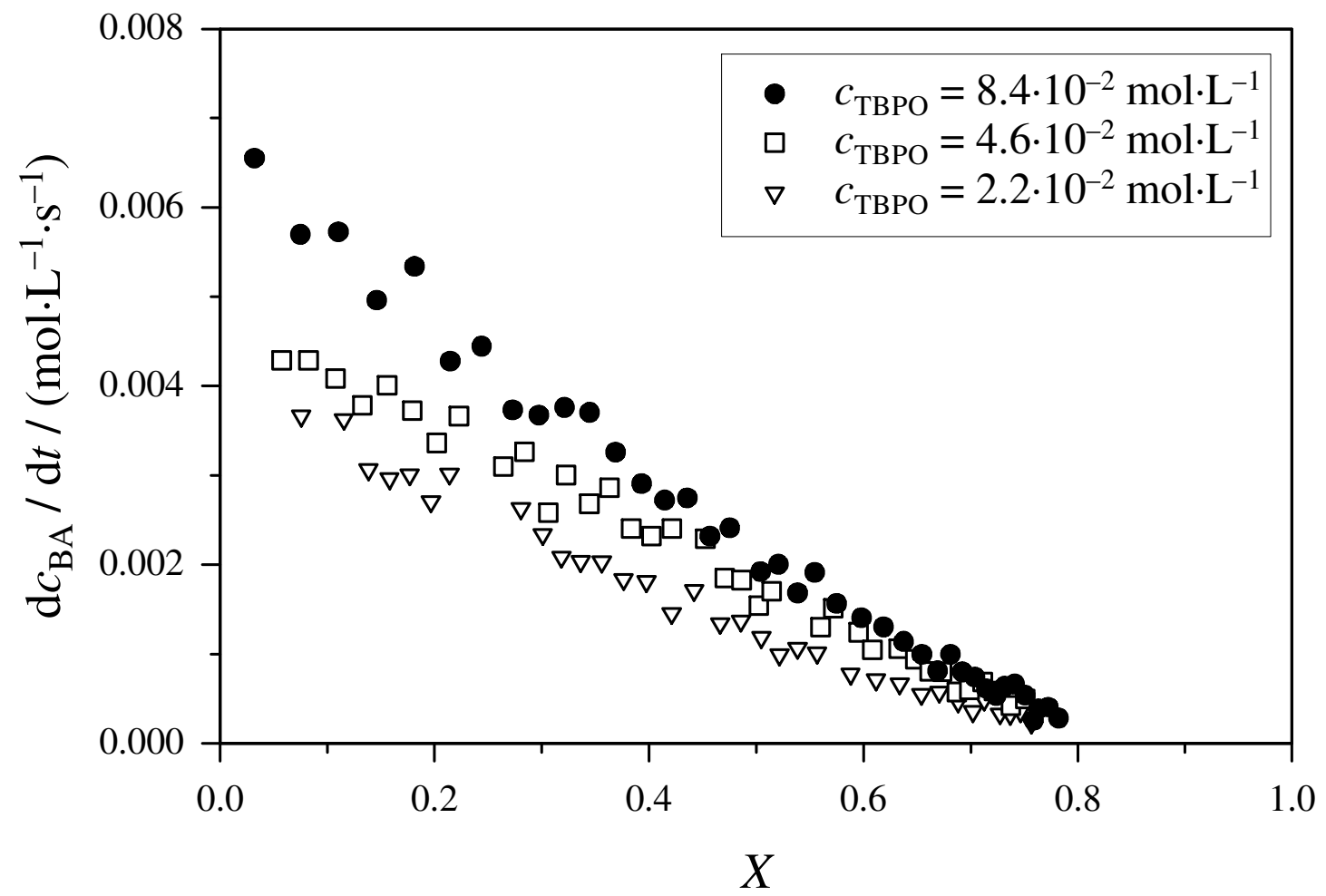

Fig. 9.17 Conversion dependence $(X)$ of overall reaction rate for chemically initiated homopolymerizations of DA in bulk at different initiator concentrations, $60{ }^{\circ} \mathrm{C}$.

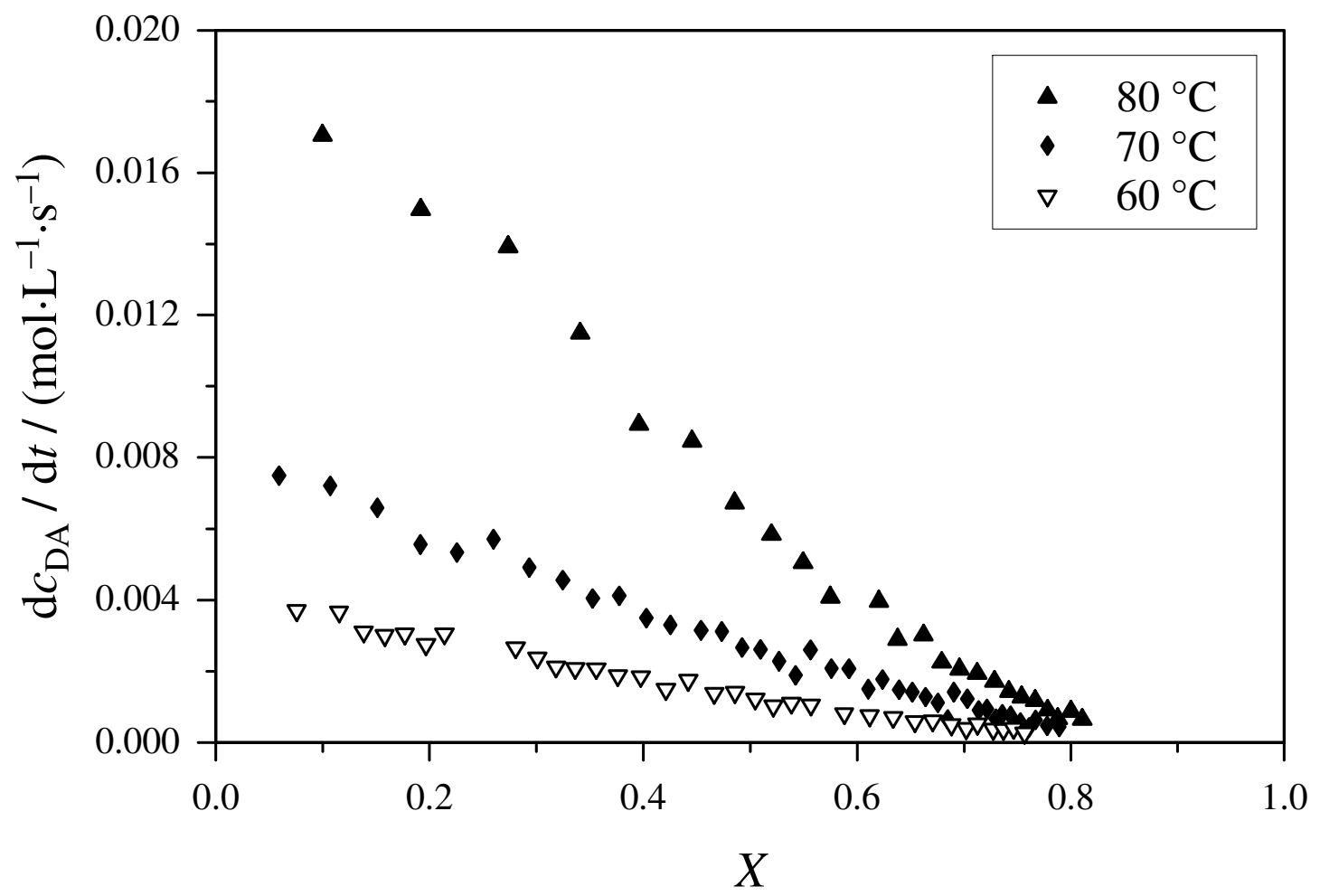

Fig. 9.18 Conversion dependence $(X)$ of overall reaction rate for chemically initiated homopolymerizations of DA in bulk at different temperatures, $c_{\mathrm{TBPO}}=2.2 \cdot 10^{-2} \mathrm{~mol} \cdot \mathrm{L}^{-1}$. 
Using the values of $f$ and $k_{\mathrm{d}}$ from Tab. 9.2 it is possible to estimate the coupled parameter $k_{\mathrm{p}} / k_{\mathrm{t}}^{0.5}$. Fig. 9.19 shows the correlation of the coupled parameter $k_{\mathrm{p}} / k_{\mathrm{t}}^{0.5}$ with monomer concentration. From Fig. 9.19 it follows that the value of $k_{\mathrm{p}} / k_{\mathrm{t}}^{0.5}$ increases linearly (over the range of concentrations considered here) as the initial monomer concentration in the solution of toluene increases.

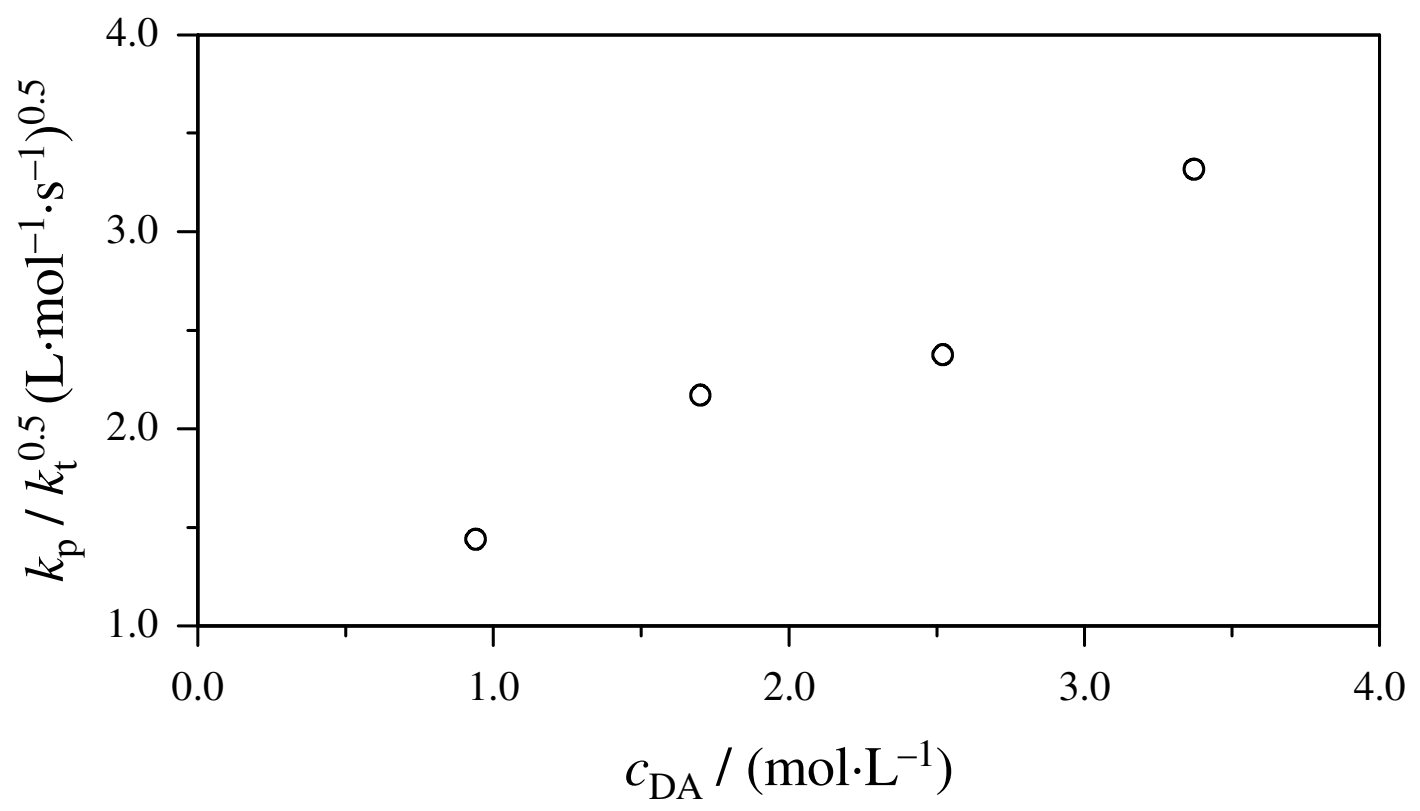

Fig. 9.19 $k_{\mathrm{p}} / k_{\mathrm{t}}^{0.5}$ as a function of DA monomer concentration in toluene solution, $c_{\mathrm{TBPO}}=8.4 \cdot 10^{-2} \mathrm{~mol} \cdot \mathrm{L}^{-1}, 60{ }^{\circ} \mathrm{C}$.

Fig. 9.20 shows the increase of the coupled parameter $k_{\mathrm{p}} / k_{\mathrm{t}}^{0.5}$ with temperature.

The increase of $k_{\mathrm{p}} / k_{\mathrm{t}}^{0.5}$ with increasing initial monomer concentration in solution and with increasing temperature is in good agreement with the BA data presented in section 9.3.1. 


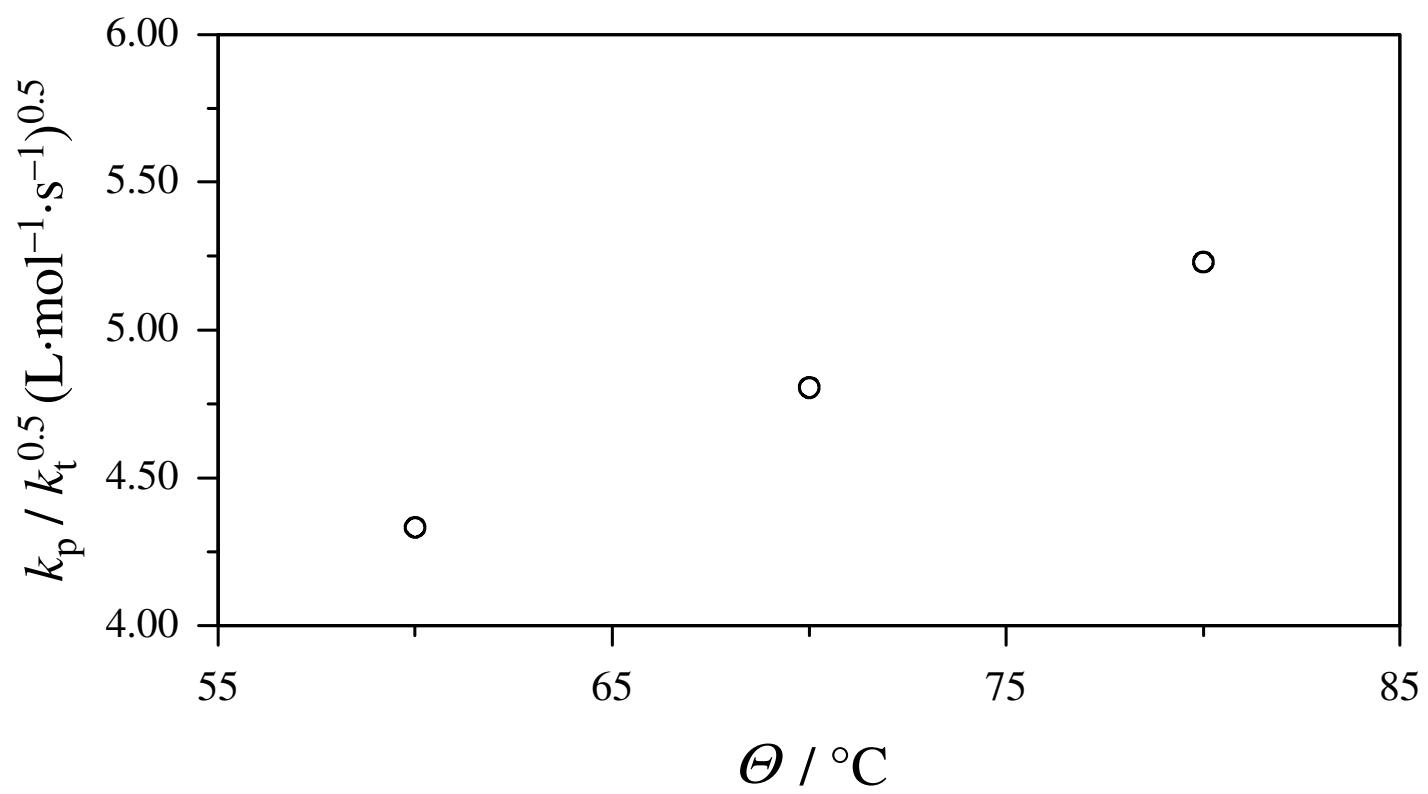

Fig. 9.20 $k_{\mathrm{p}} / k_{\mathrm{t}}^{0.5}$ as a function of temperature for DA bulk polymerizations, $c_{\mathrm{TBPO}}=2.2 \cdot 10^{-2} \mathrm{~mol} \cdot \mathrm{L}^{-1}$.

Fig. 9.21 shows the variation of the overall polymerization rate as a function of monomer concentration. The slope of this plot is consistent with monomer reaction order of 1.59. "Classical" theory would predict unity for such exponent.

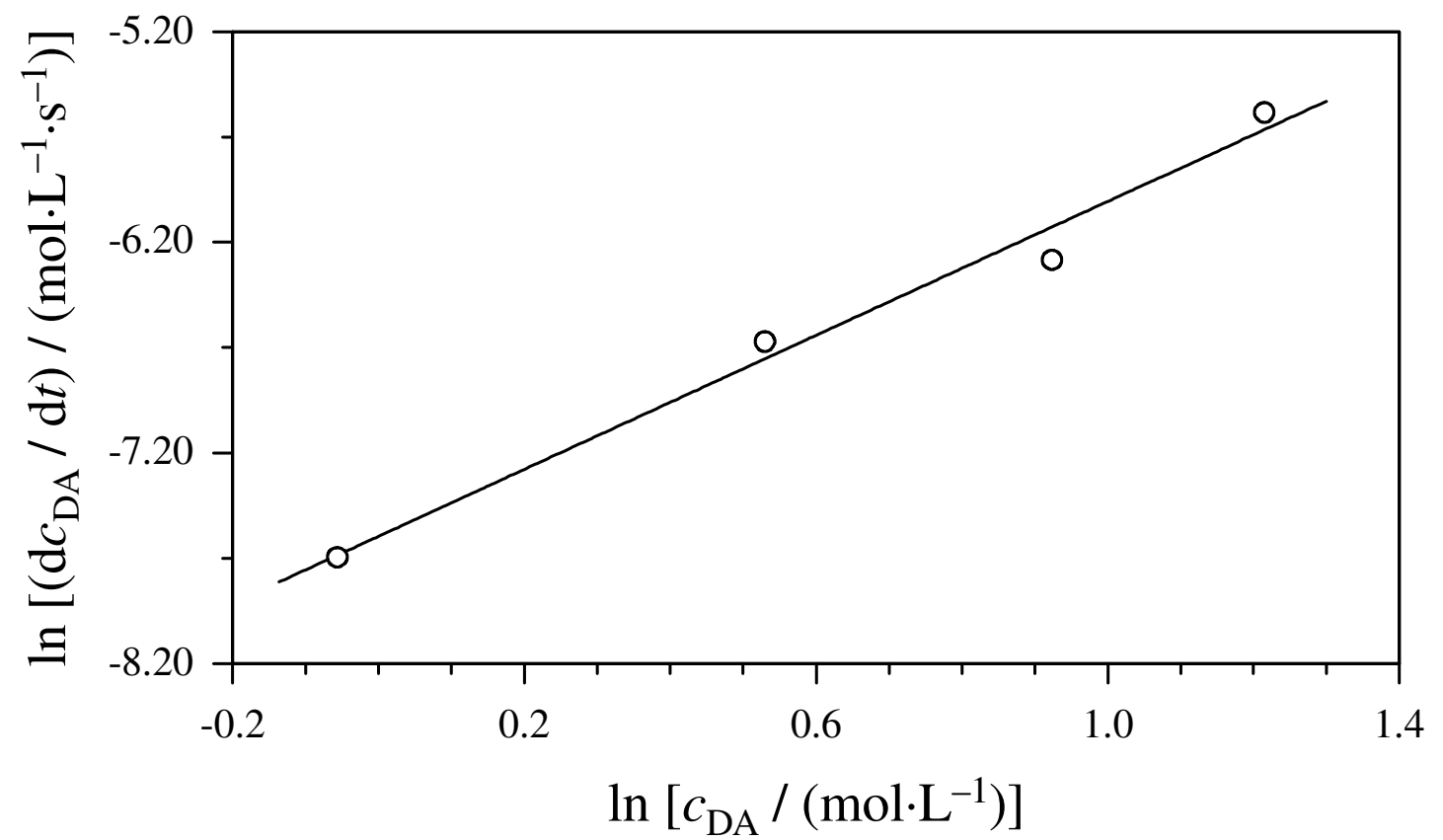

Fig. 9.21 Double logarithmic plot of overall reaction rate of DA vs. monomer concentration in toluene solution at 30 per cent monomer conversion, $c_{\mathrm{TBPO}}=8.6 \cdot 10^{-2} \mathrm{~mol} \cdot \mathrm{L}^{-1}, 60^{\circ} \mathrm{C}$. 


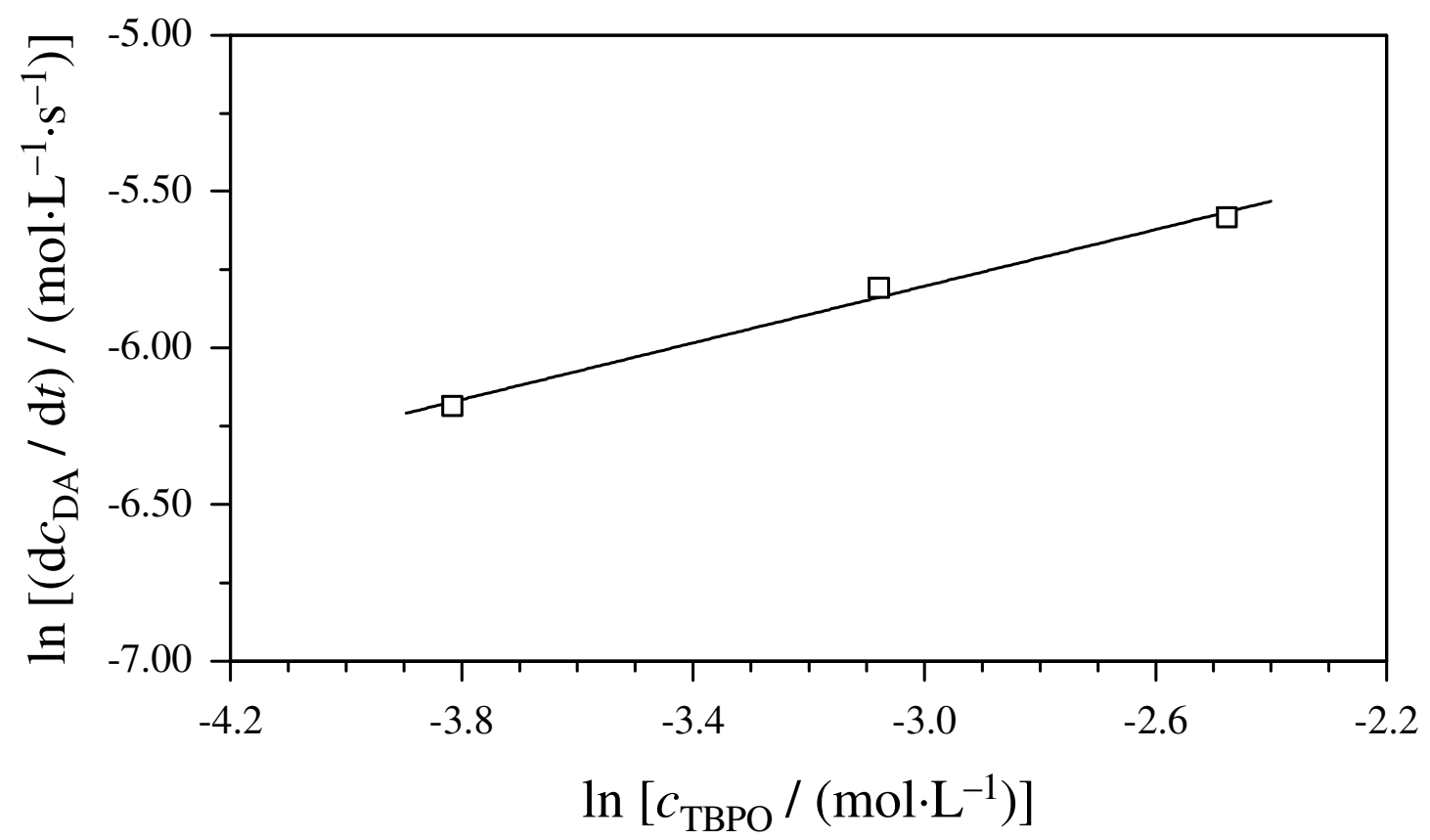

Fig. 9.22 Double logarithmic plot of overall reaction rate of DA in bulk vs. initiator concentration at 30 per cent monomer conversion, $60{ }^{\circ} \mathrm{C}$.

From the slope in Fig. 9.22, where the overall polymerization rate as a function of initiator concentration is presented, an reaction order of 0.45 for TBPO is obtained. This is very close to the theoretically predicted value of 0.5 . The overall polymerization rate of DA is proportional to the monomer and initiator concentrations as follows:

$$
-\frac{\mathrm{d} c_{\mathrm{DA}}}{\mathrm{d} t} \propto c_{\mathrm{DA}}^{1.59} \cdot c_{\mathrm{TBPO}}^{0.45}
$$

The obtained dependence of the overall reaction rate of DA on the monomer and initiator concentrations is close to the case II.b of the model proposed in the section 9.2. In this case the rate of propagation of tertiary radicals is much slower than the rate of their production with an assumption $k_{\mathrm{p} 2} \cdot c_{\mathrm{M}} \gg k_{\mathrm{t}} \cdot c_{\mathrm{R}}$.

For the estimate of the intramolecular transfer coefficient, $k_{\mathrm{bb}}$, Eq. 9.6a can be used. The ratio of $c_{\mathrm{R}_{2}} / c_{\mathrm{R}}$ in Eq. 9.6a was obtained from laser-initiated ESR experiments presented in section 7.2.2 as well from ESR measurements of DA polymerization initiated by thermal decomposition of TBPO at $60^{\circ} \mathrm{C}$. $k_{\mathrm{p} 2}$ values were assumed to be close to the propagation rate coefficients of DAD (Section 8). 


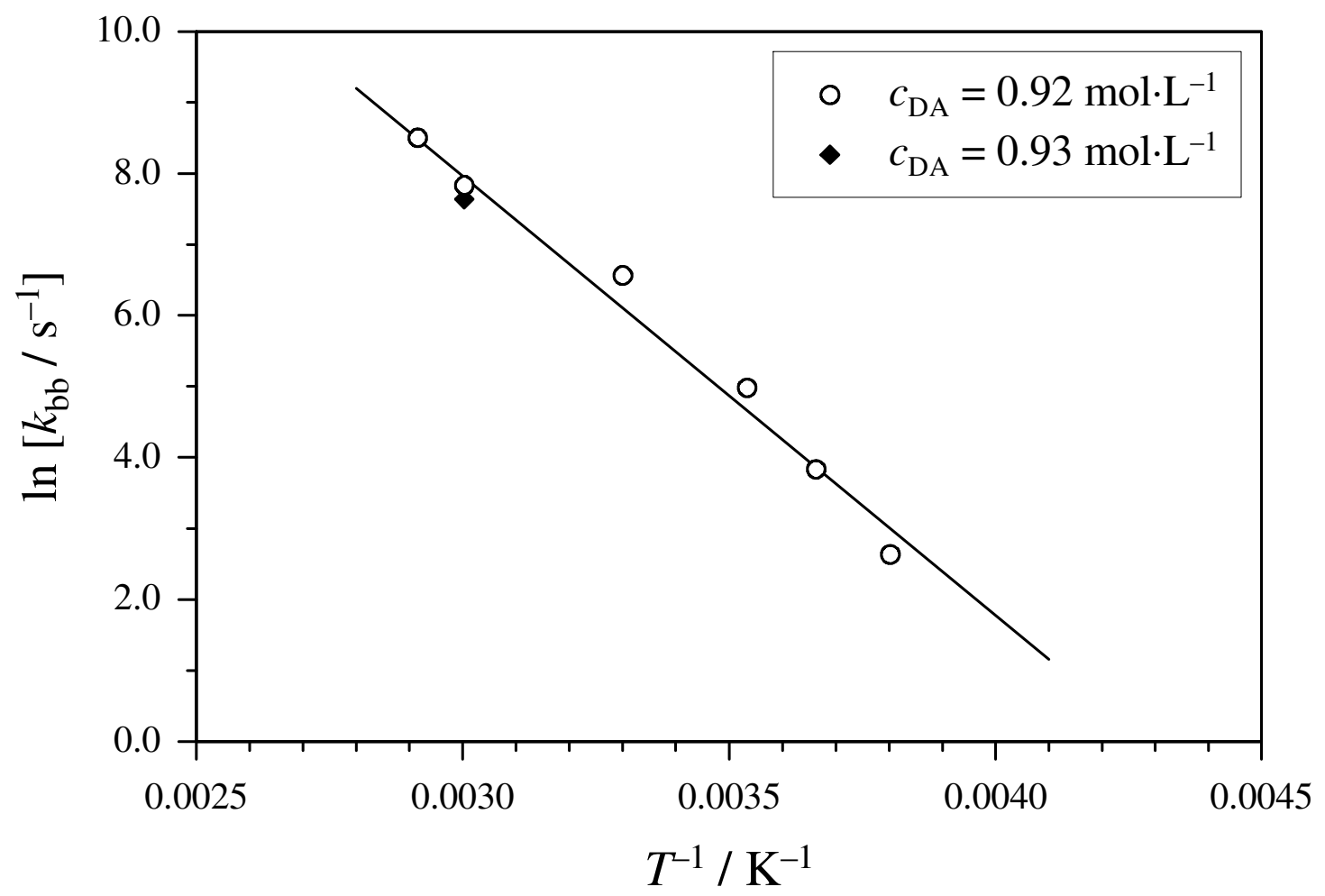

Fig. 9.23 Temperature dependence of the intramolecular transfer coefficient, $k_{\mathrm{bb}}$, obtained via Eq. 9.6a for DA free-radical homopolymerization at ambient pressure. The ratio of $c_{\mathrm{R}_{2}} / c_{\mathrm{R}}$ in Eq. 9.6a was obtained from laser-initiated ESR experiments presented in section 7.2.2 (open symbols) and from DA polymerization initiated by thermal decomposition of TBPO (full symbol).

The Arrhenius expression from Fig. 9.23 for $k_{\mathrm{bb}}$ of DA is represented by Eq. 9.14:

$$
\ln \left[k_{\mathrm{bb}} / \mathrm{s}^{-1}\right]=26.52-6266 \cdot\left(T^{-1} / \mathrm{K}^{-1}\right)
$$

(DA homopolymerization at ambient pressure, $-20 \leq \Theta /{ }^{\circ} \mathrm{C} \leq 70$ ).

From the slope to the straight line in Fig. 9.23, the activation energy for $k_{\mathrm{bb}}$ of DA is estimated to be: $E_{\mathrm{A}}\left(k_{\mathrm{bb}}\right)=(52.1 \pm 9.6) \mathrm{kJ} \cdot \mathrm{mol}^{-1}$. This value is very close to the value of the activation energy for the intramolecular transfer reaction obtained for BA.

\section{PREDICI Simulations}

In Tab. 9.4 the frequency factors and activation energies of the rate coefficients used in the PREDICI ${ }^{\circledR}$ simulations for the two types of radicals are given. Values of $k_{\mathrm{p} 1}$ were estimated from the data obtained by PLP-SEC for lower temperatures [29], the values of termination rate coefficient $k_{\mathrm{t}}^{1,1}$ were extrapolated from SP-PLP-NIR experiments, performed at 1000 bar, 
to ambient pressure with an activation volume of $V_{40}^{\neq}{ }^{\circ} \mathrm{C}=20.5 \mathrm{~cm}^{3} \cdot \mathrm{mol}^{-1}$ [27]. The frequency factor and activation energy of the propagation rate coefficient of the tertiary radicals were taken from the PLP values of the dodecyl acrylate dimer (Section 8) that yields a tertiary radical similar to that generated after chain transfer to polymer in DA homopolymerization. There is no literature data for termination rate coefficients of mid-chain radicals produced in the intramolecular chain transfer reaction. Hence, values from the family of itaconates (that also yields tertiary radicals) were taken [24].

\begin{tabular}{cccc}
\hline DA & $A / \mathrm{L} \cdot \mathrm{mol}^{-1} \cdot \mathrm{s}^{-1}$ & $E_{\mathrm{A}} / \mathrm{kJ} \cdot \mathrm{mol}^{-1}$ & Source \\
\hline Secondary radical & $1.79 \cdot 10^{7}$ & 17.0 & {$[29]$} \\
Propagation, $k_{\mathrm{p} 1}$ & $1.4 \cdot 10^{7}$ & 4.6 & {$[27]$} \\
Termination, $k_{\mathrm{t}}^{1,1}$ & $3.3 \cdot 10^{11}$ & 51.5 & this work \\
Backbiting, $k_{\mathrm{bb}}$ & & & \\
Mid-chain radical & $4.1 \cdot 10^{7}$ & 35.4 & this work \\
Propagation, $k_{\mathrm{p} 2}$ & $1.6 \cdot 10^{9}$ & 23.0 & {$[24]$} \\
Termination, $k_{\mathrm{t}}^{2,2}$ & & & \\
\hline
\end{tabular}

Tab. 9.4 Kinetic parameters for PREDICI® simulations.

Fig. 9.24 presents the simulated conversion dependence of the overall reaction rate in comparison with measured data. 


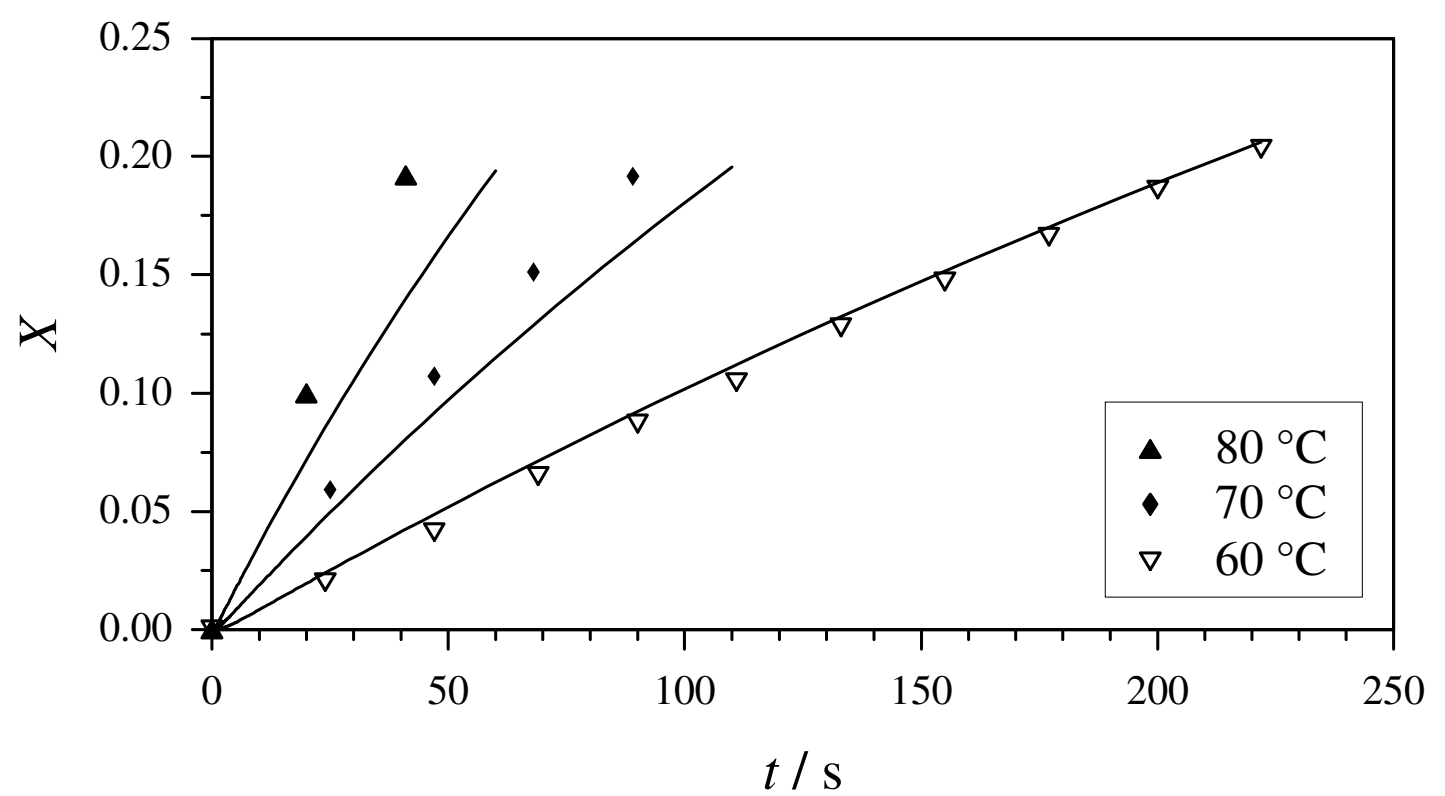

Fig. 9.24 Simulated (lines) and measured (points) conversion dependence $(X)$ of the overall reaction rate for chemically initiated bulk homopolymerization of DA at different temperatures, $c_{\mathrm{TBPO}}=2.2 \cdot 10^{-2} \mathrm{~mol} \cdot \mathrm{L}^{-1}$.

As it can be seen in Fig. 9.23, proposed kinetic schema describes the bulk homopolymerization of DA up to 10 per cent of conversion well, particularly at $60{ }^{\circ} \mathrm{C}$.

\subsubsection{Chemically INitiated Polymerization of DMA}

In this section data obtained from DMA homopolymerization initiated by thermal decomposition of TBPO will be presented. First, it will be shown that at low conversion the homopolymerization of DMA is close to the ideal kinetics. Further, values of $k_{\mathrm{t}}$ obtained from thermally initiated homopolymerization of DMA in comparison with values of termination rate coefficients obtained via the SP-PLP-NIR and the SP-PLP-SEC (from section 6.3.1) techniques will be given. At the end of this section PREDICI ${ }^{\circledR}$ simulations for thermally initiated homopolymerization of DMA will be shown.

Bulk polymerization of DMA was investigated by several authors [30,31,32]. The absence of the gel effect with increasing the size of the alkyl ester group in the methacrylate monomers was reported in literature [33], where a clear gel effect is a typical kinetic property of methyl methacrylate polymerization [34,35]. Lazar et al. [32] explained a considerable suppressing of the gel effect in the homopolymerization of DMA as being due to the transfer reaction of the propagation radical to the alkyl ester group. 
For detailed investigations into kinetics of DMA homopolymerization studies at ambient pressure and over the temperature range 60 to $80{ }^{\circ} \mathrm{C}$ were carried out. Toluene was used as the solvent for determination of the reaction order of DMA.

Conversion curves of bulk DMA homopolymerization, shown in Figs. 9.25 and 9.26 as a function of time, do not show an acceleration (over the range of initiator concentrations and temperatures considered here) of the polymerization rate, as that is observed for methyl methacrylate polymerization [34]. A linear increase in conversion in the beginning of polymerization is observed.

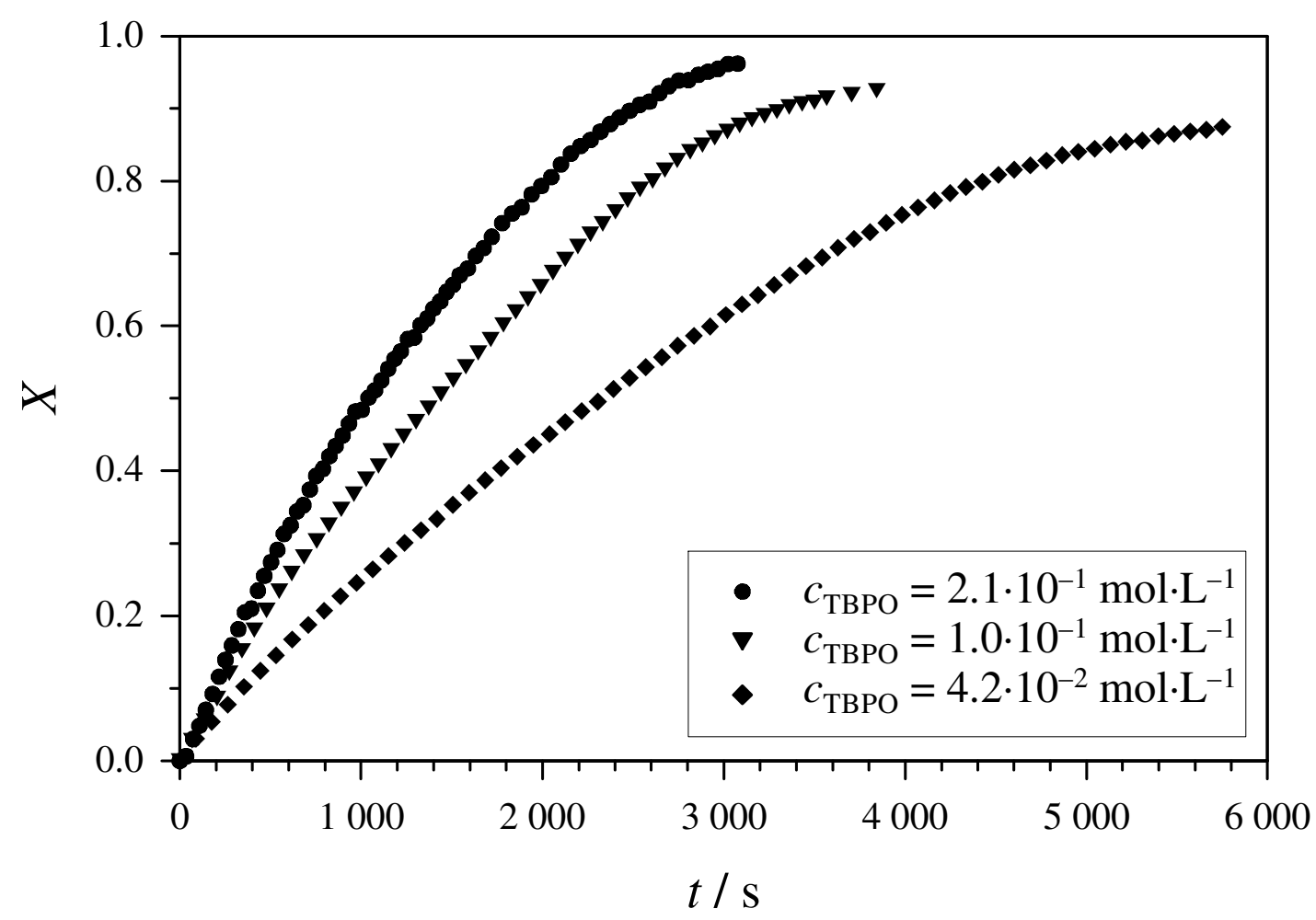

Fig. 9.25 Conversion vs. time plots for chemically initiated bulk homopolymerization of DMA at different initiator concentrations, $70^{\circ} \mathrm{C}$. 


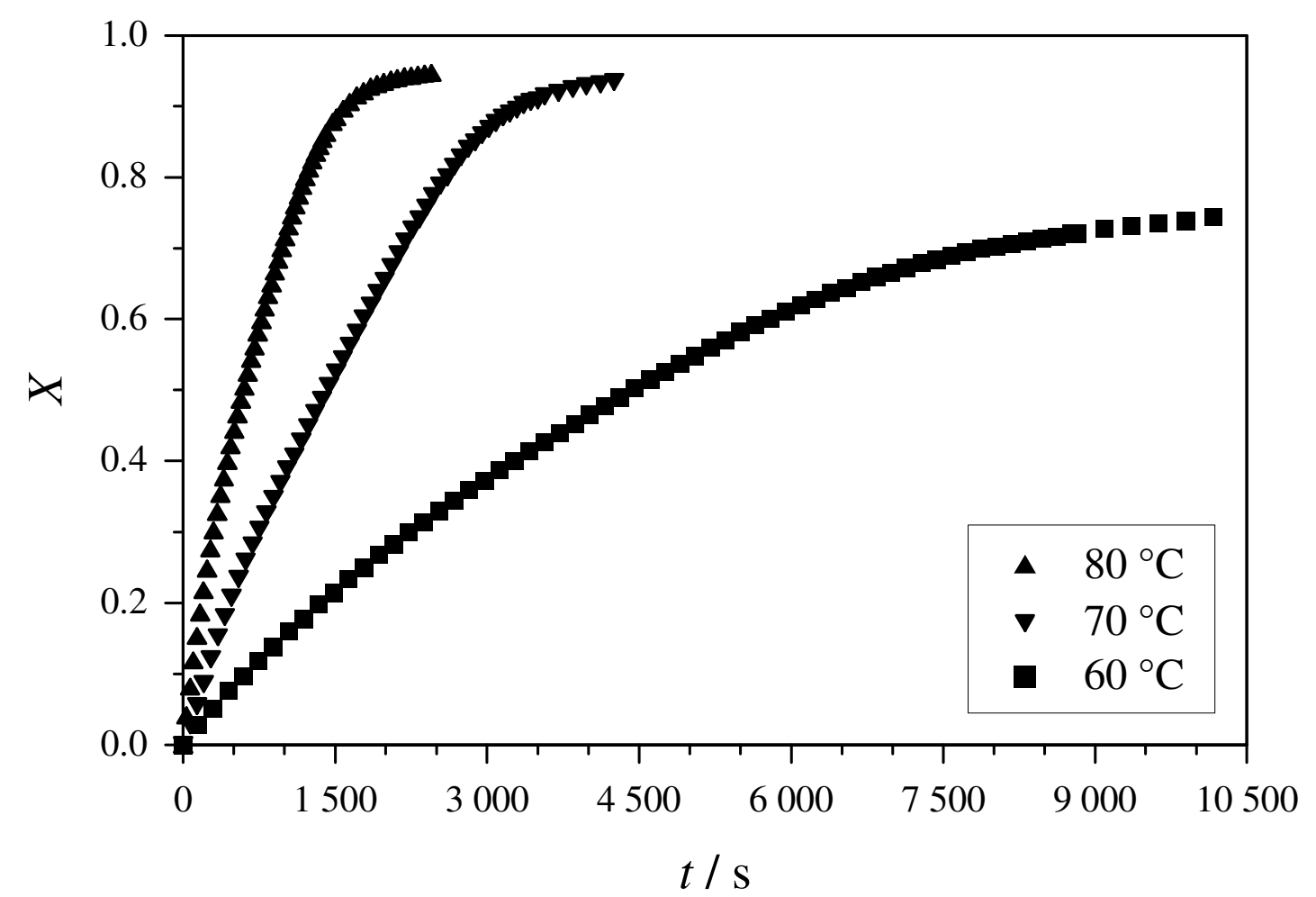

Fig. 9.26 Conversion dependence $(X)$ of overall reaction rate for chemically initiated bulk homopolymerization of DMA at different temperatures, $c_{\mathrm{TBPO}}=1.0 \cdot 10^{-1} \mathrm{~mol} \cdot \mathrm{L}^{-1}$.

From overall polymerization rate as a function of monomer conversion, presented in Figs. 9.27 and 9.28, it follows that the reaction rate decreases up to approximately 30 per cent monomer conversion, then the reduction in reaction rate becomes slower and at conversions higher than 70 per cent falls to zero. 


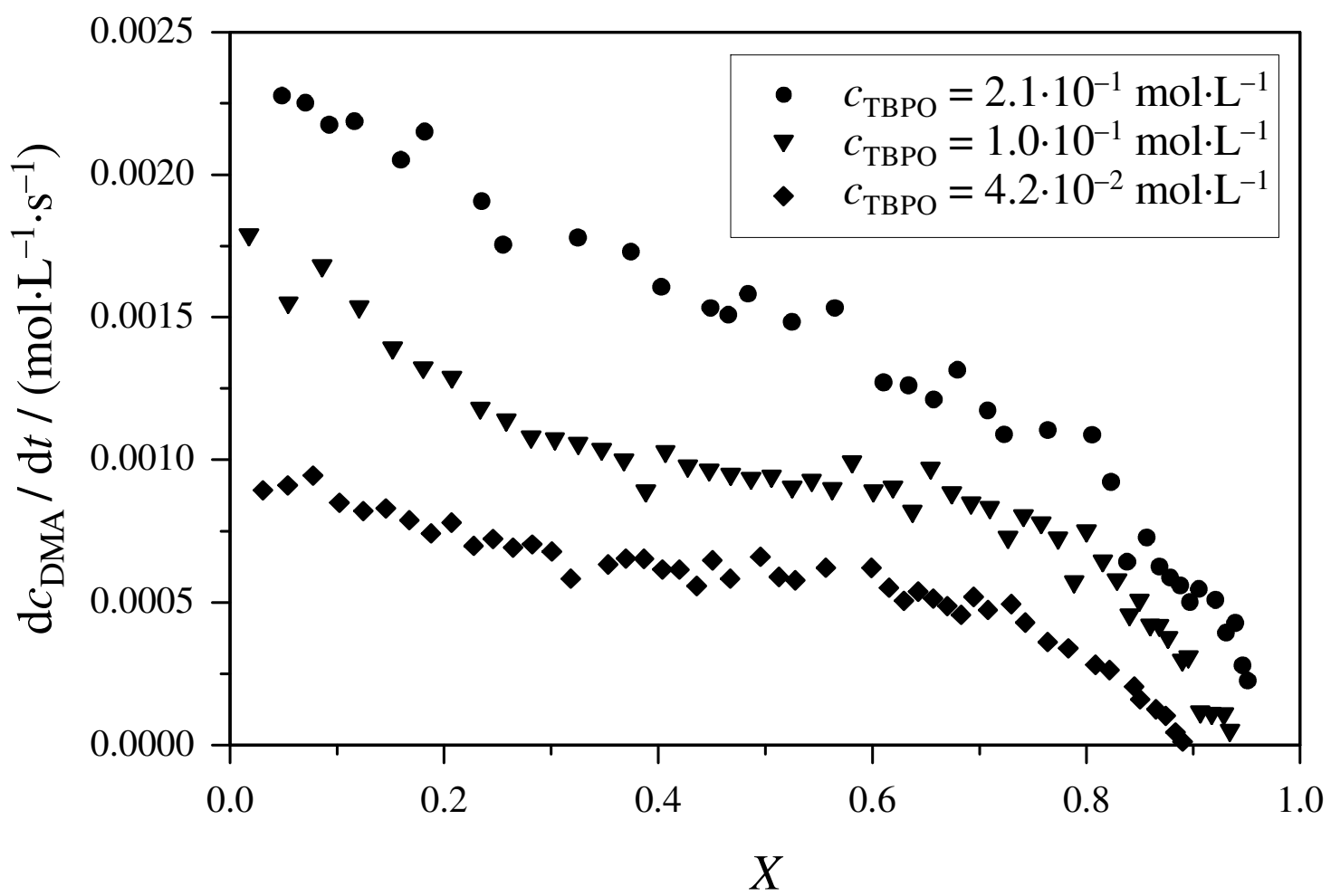

Fig. 9.27 Conversion dependence $(X)$ of the overall reaction rate for chemically initiated bulk homopolymerizations of DMA at different initiator concentrations, $70{ }^{\circ} \mathrm{C}$.

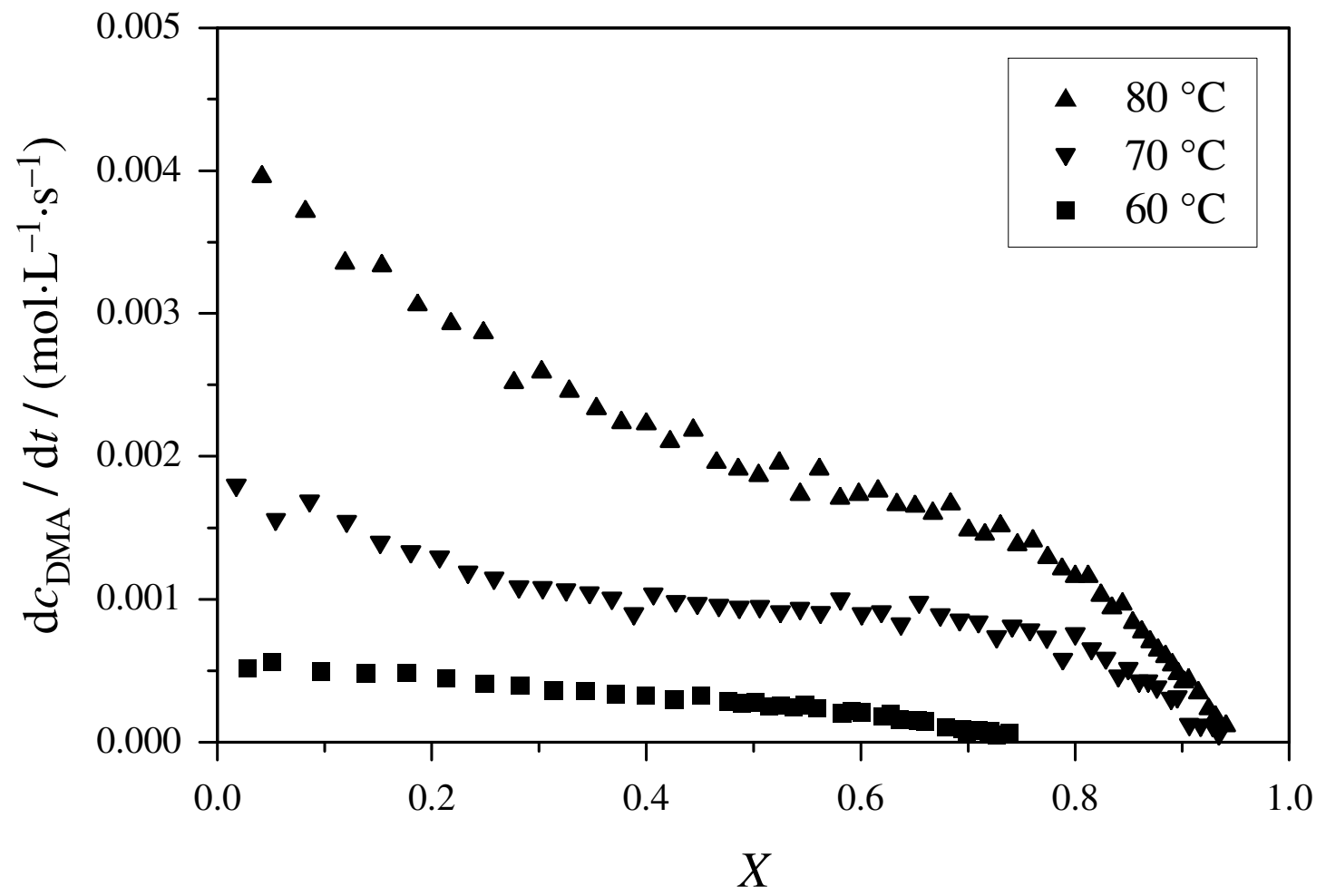

Fig. 9.28 Conversion dependence $(X)$ of the overall reaction rate for chemically initiated bulk homopolymerizations of DMA at different temperatures, $c_{\mathrm{TBPO}}=1.0 \cdot 10^{-1} \mathrm{~mol} \cdot \mathrm{L}^{-1}$. 
Figs. 9.25 and 9.26 show that the initial concentration of monomer in solution and reaction temperature have an large influence on the rate of polymerization. As expected, the experiments with higher TBPO concentration are "faster" than the ones with lower initiator concentration (Fig. 9.27). With increasing temperature, the rate of polymerization increases as well (Fig. 9.28).

For estimates of the coupled parameter $k_{\mathrm{p}} / k_{\mathrm{t}}^{0.5}$ it is necessary to choose a value for the constants $f$ and $k_{\mathrm{d}}$. Using the values of $f$ and $k_{\mathrm{d}}$ for TBPO from Tab. 9.2 it becomes possible to obtain a value of the coupled parameter $k_{\mathrm{p}} / k_{\mathrm{t}}^{0.5}$. Fig. 9.29 shows correlation of the coupled parameter $k_{\mathrm{p}} / k_{\mathrm{t}}^{0.5}$ as a function of monomer concentration.

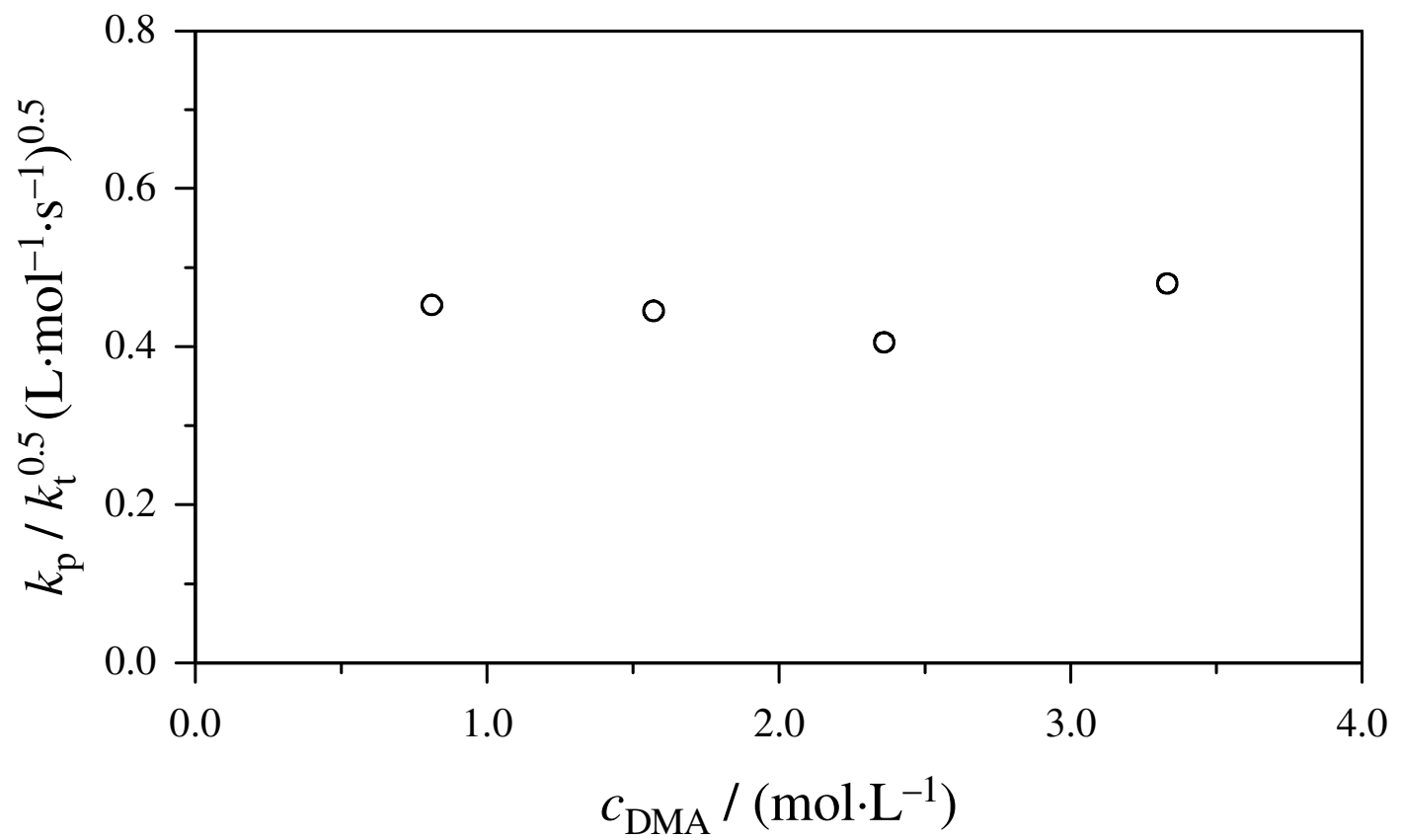

Fig. 9.29 $k_{\mathrm{p}} / k_{\mathrm{t}}^{0.5}$ as a function of DMA monomer concentration in toluene solution, $c_{\mathrm{TBPO}}=1.0 \cdot 10^{-1} \mathrm{~mol} \cdot \mathrm{L}^{-1}, 70{ }^{\circ} \mathrm{C}$.

It follows from Fig. 9.29 that the value of $k_{\mathrm{p}} / k_{\mathrm{t}}^{0.5}$ is independent of the initial monomer concentration in the solution of toluene. This observation differs from the one made for DA homopolymerization, presented in section 9.3.2. For DA the coupled parameter $k_{\mathrm{p}} / k_{\mathrm{t}}^{0.5}$ increases linearly with increasing the initial monomer concentration in the toluene solution. The value of $k_{\mathrm{p}} / k_{\mathrm{t}}^{0.5}$, shown in Fig. 9.30, slightly increases with temperature. 


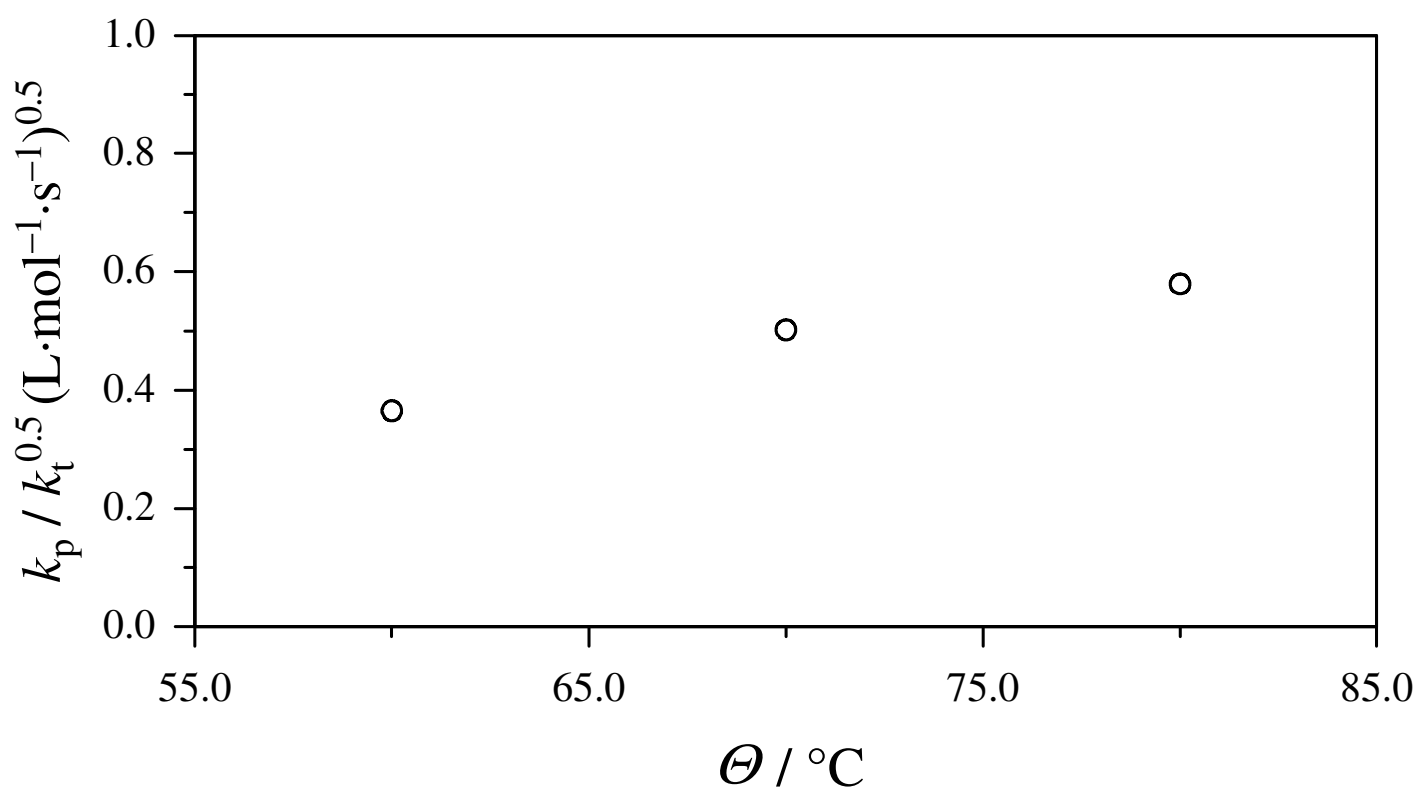

Fig. 9.30 $k_{\mathrm{p}} / k_{\mathrm{t}}^{0.5}$ as a function of temperature, DMA bulk polymerization, $c_{\mathrm{TBPO}}=1.0 \cdot 10^{-1} \mathrm{~mol} \cdot \mathrm{L}^{-1}$.

Fig. 9.31 shows the overall polymerization rate as a function of the DMA monomer concentration. From the slope of this plot the reaction order for DMA is determined to be 0.96. This value is in good agreement with the value predicted for ideal polymerization.

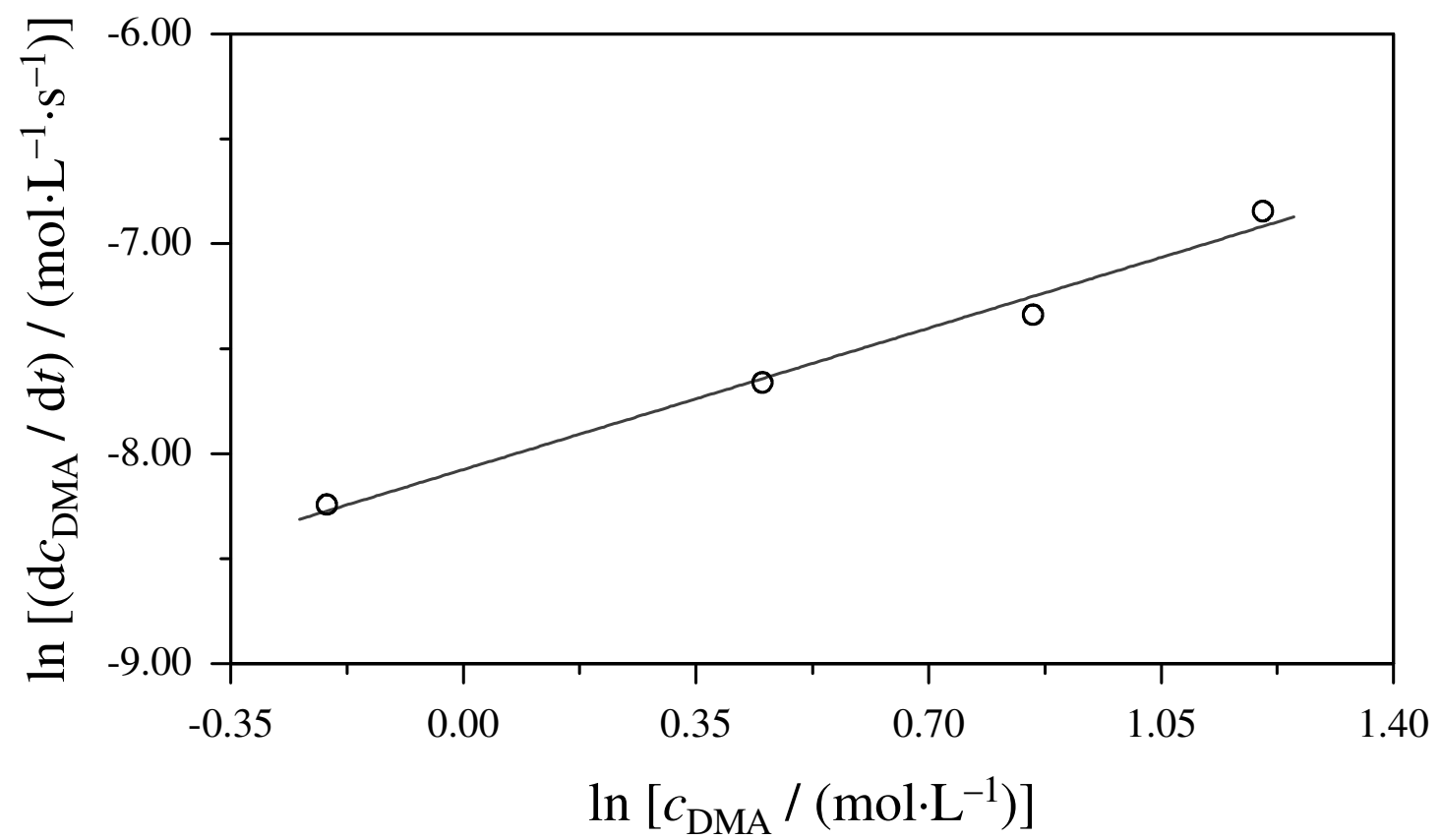

Fig. 9.31 Double logarithmic plot of overall reaction rate of DMA vs. monomer concentration in toluene solution at 30 per cent of conversion, $c_{\mathrm{TBPO}}=1.0 \cdot 10^{-1} \mathrm{~mol} \cdot \mathrm{L}^{-1}, 70{ }^{\circ} \mathrm{C}$. 


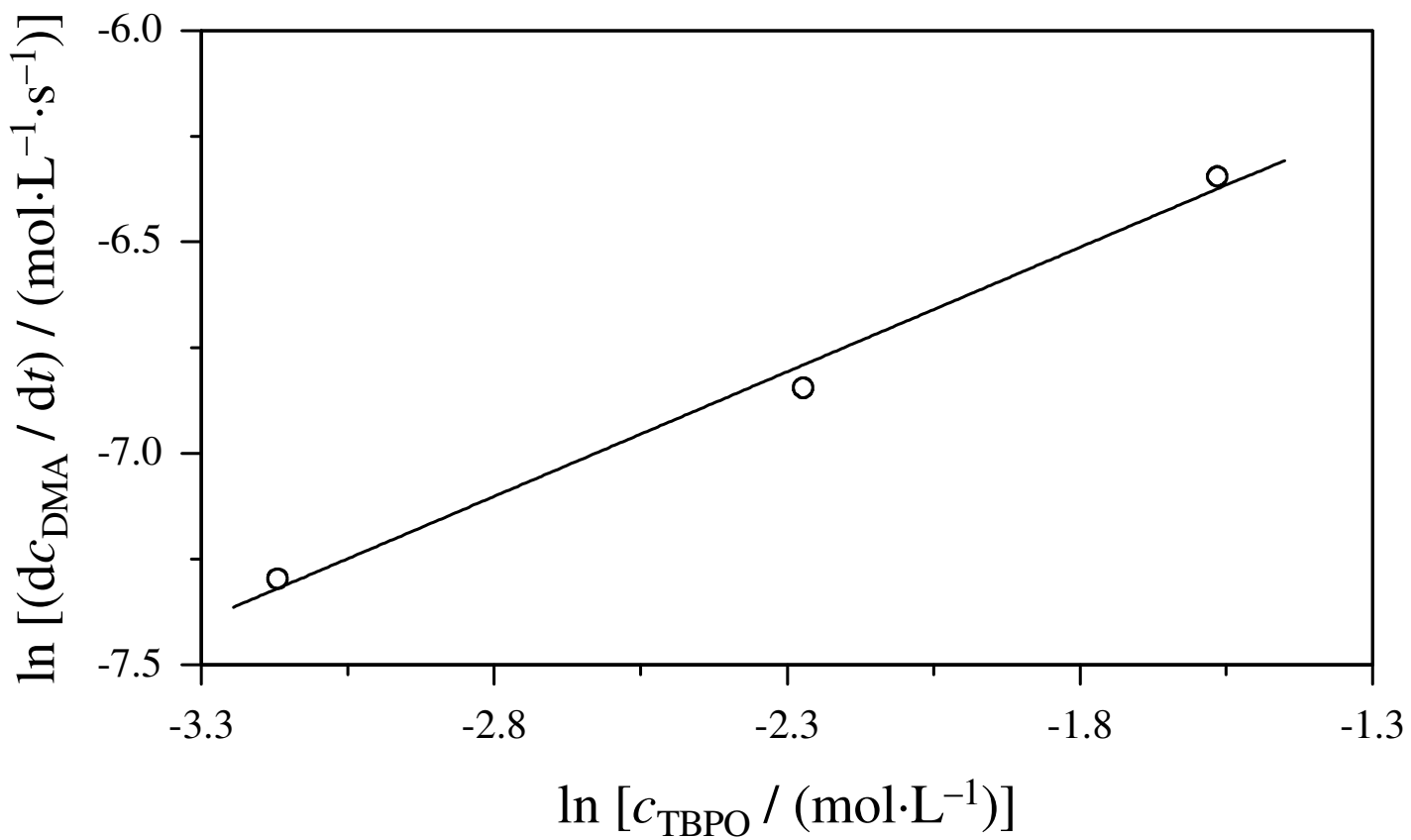

Fig. 9.32 Double logarithmic plot of overall reaction rate of DMA in bulk vs. initiator concentration at 30 per cent of conversion, $70{ }^{\circ} \mathrm{C}$.

From the slope in Fig. 9.32, where the overall polymerization rate as a function of the TBPO initiator concentration is presented, the exponent for initiator is found to be 0.59 . This is rather close to the expected value of 0.5 . The overall polymerization rate of DMA is proportional to the monomer and initiator concentrations as follows:

$$
-\frac{\mathrm{d} c_{\mathrm{DMA}}}{\mathrm{d} t} \propto c_{\mathrm{DMA}}^{0.96} \cdot c_{\mathrm{TBPO}}^{0.59}
$$

From polymerizations initiated by thermally decomposing chemical initiator the coupled parameter $k_{\mathrm{p}} / k_{\mathrm{t}}^{0.5}$ using Eq. 9.1 can be determined. Thus termination rate coefficients can be calculated with propagation rate coefficients taken from PLP-SEC experiments [36]:

$$
\ln \left[k_{\mathrm{p}} /\left(\mathrm{L} \cdot \mathrm{mol}^{-1} \cdot \mathrm{s}^{-1}\right)\right]=14.5-2444 \cdot\left(T^{-1} / \mathrm{K}^{-1}\right)
$$

(DMA bulk homopolymerization at ambient pressure, $30 \leq \Theta /{ }^{\circ} \mathrm{C} \leq 90$ ).

Buback et al. [37] pointed out that the values of termination rate coefficients from a nonsteady state experiment (such as a single pulse laser experiment) should not be assumed to hold for a corresponding steady state experiment due to the difference in the radical chain length distribution. Therefore, it was recommended to compare $k_{\mathrm{t}}$ from steady-state experiments with $k_{\mathrm{t}}$ from non-steady state measurements. The two values of $k_{\mathrm{t}}$ thus obtained will give some idea of the variation of $k_{\mathrm{t}}$ which can be expected due to variation of the radical chain length distribution. 
Fig. 9.33 makes a comparison of termination rate coefficients obtained from steady-state and non-steady state experiments. Full squares in Fig. 9.33 correspond to the values of $\left\langle k_{\mathrm{t}}\right\rangle$ calculated via Eq. 3.6 from the SP-PLP-ESR data obtained at $0{ }^{\circ} \mathrm{C}$ and ambient pressure (Section 6.3.1) and than extrapolated to $70{ }^{\circ} \mathrm{C}$ with $E_{\mathrm{A}}=17 \mathrm{~kJ} \cdot \mathrm{mol}^{-1}$. The values of $<k_{\mathrm{t}}>$, given in Fig. 9.33 as full circles, correspond to the SP-PLP-NIR data and were calculated via Eq. 3.11 for the chain-length interval $0<i<1600$. The primary SP-PLP-NIR results were obtained at $70{ }^{\circ} \mathrm{C}$ and 5 bar as described in experimental section and then extrapolated to ambient pressure with $V_{30}^{\neq}{ }^{\circ} \mathrm{C}=10.8 \mathrm{~cm}^{3} \cdot \mathrm{mol}^{-1}[27]$.

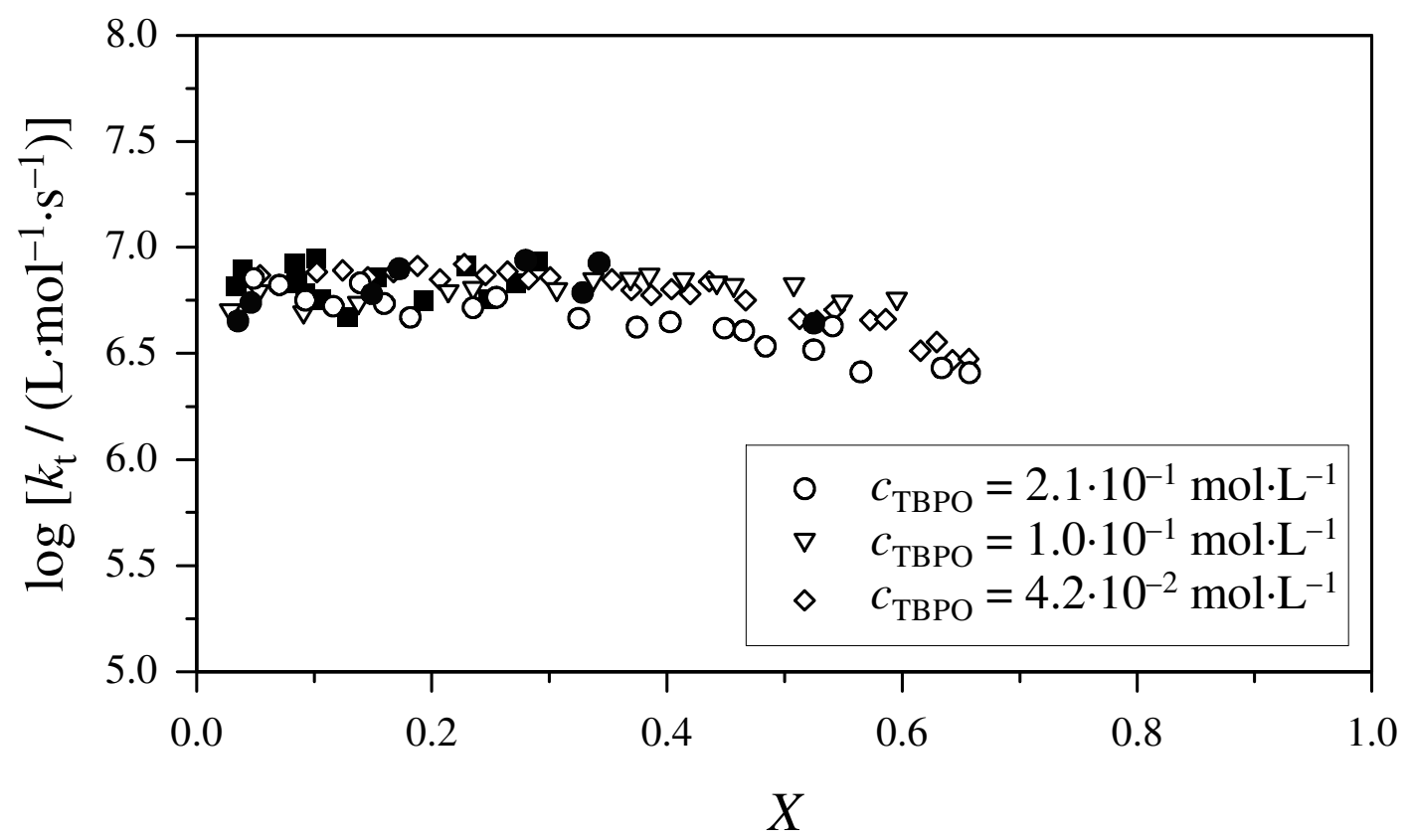

Fig. 9.33 Conversion dependence of $k_{\mathrm{t}}$ for chemically initiated bulk homopolymerization of DMA (open symbols) in comparison with the values of $\left\langle k_{\mathrm{t}}\right\rangle$, obtained via SP-PLP-NIR, $c_{\text {MMMP }}=1.1 \cdot 10^{-2} \mathrm{~mol} \cdot \mathrm{L}^{-1}$ (full circles), and $<k_{\mathrm{t}}>$ from SP-PLPESR experiments (full squares). All data points refer to $70^{\circ} \mathrm{C}$ and ambient pressure.

Fig. 9.33 shows that the values of termination rate coefficients calculated from chemically initiated homopolymerizations of DMA are close to the ones obtained via SP-PLP-NIR. The values of $k_{\mathrm{t}}$ extrapolated from the SP-PLP-ESR data measured at $0{ }^{\circ} \mathrm{C}$ and ambient pressure are close to the SP-PLP-NIR results. 


\section{PREDICI@ Simulations}

PREDICI ${ }^{\circledR}$ simulations were carried out in order to test whether the kinetic scheme of DMA bulk homopolymerization is close to ideal kinetics. For $60{ }^{\circ} \mathrm{C}$ and ambient pressure the following rate coefficients for bulk homopolymerization of DMA were used: $k_{\mathrm{p}}=1600 \mathrm{~L} \cdot \mathrm{mol}^{-1} \cdot \mathrm{s}^{-1}$ and $k_{\mathrm{t}}=6.37 \cdot 10^{6} \mathrm{~L} \cdot \mathrm{mol}^{-1} \cdot \mathrm{s}^{-1}$. The termination rate coefficient was calculated from the data shown in Fig. 9.33 for the homopolymerization of DMA initiated by thermal decomposition of TBPO over the monomer conversion up to 40 per cent. Parameters used for TBPO are given in Tab. 9.2.

In Fig. 9.34 the simulated conversion dependence of the overall reaction rate is shown in comparison with measured data. It follows from this graph that, at least up to 20 per cent of conversion, the kinetics of bulk polymerization of DMA is close to ideal.

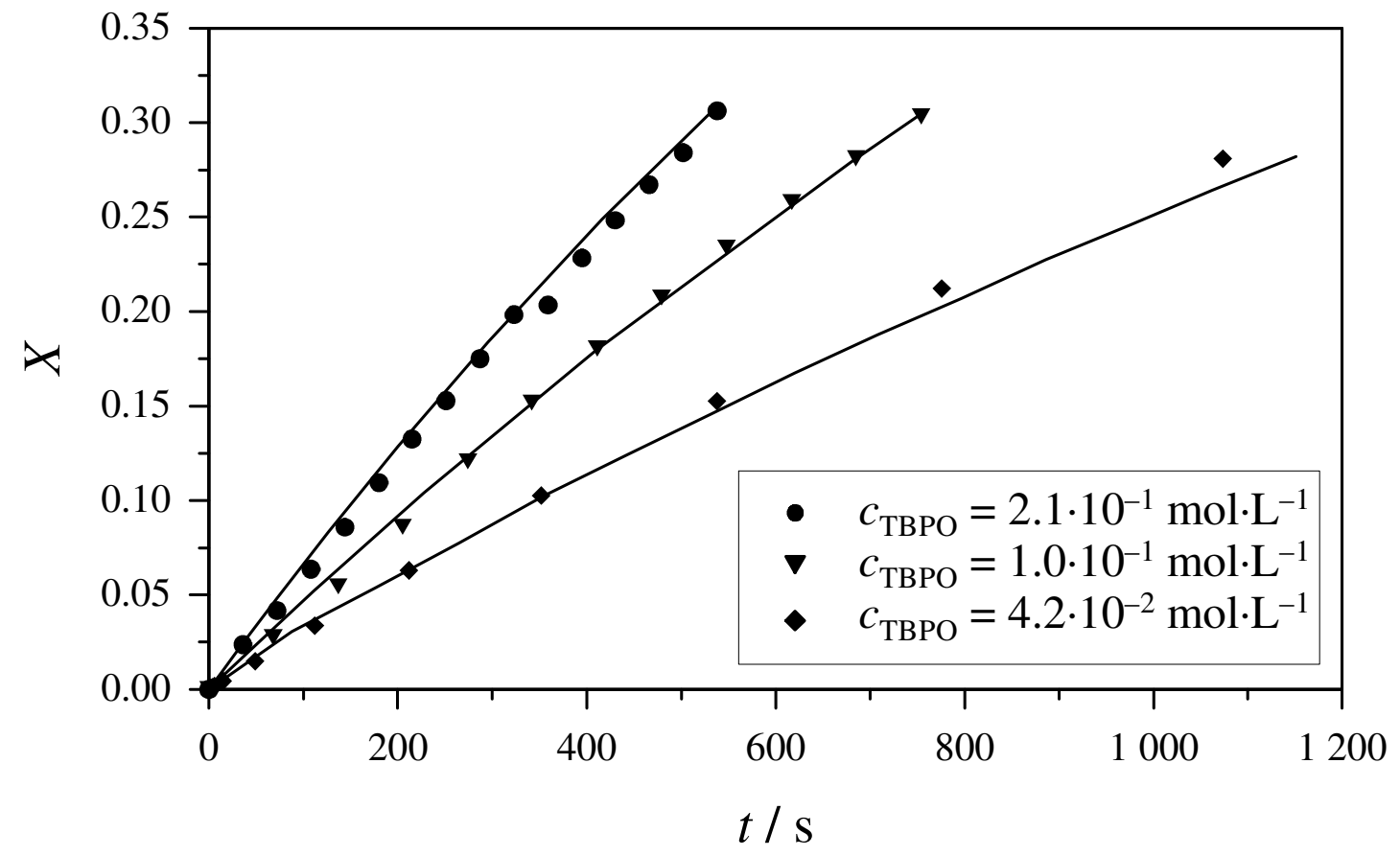

Fig. 9.34 Simulated via PREDICI ${ }^{\circledR}$ (lines) and measured (points) conversion dependence $(X)$ of the overall reaction rate for chemically initiated bulk homopolymerization of DMA at different initiator concentrations, $70{ }^{\circ} \mathrm{C}$.

Fig. 9.35 shows the molecular weight distribution of polyDMA obtained by SEC in comparison with MWD simulated via PREDICI®. 


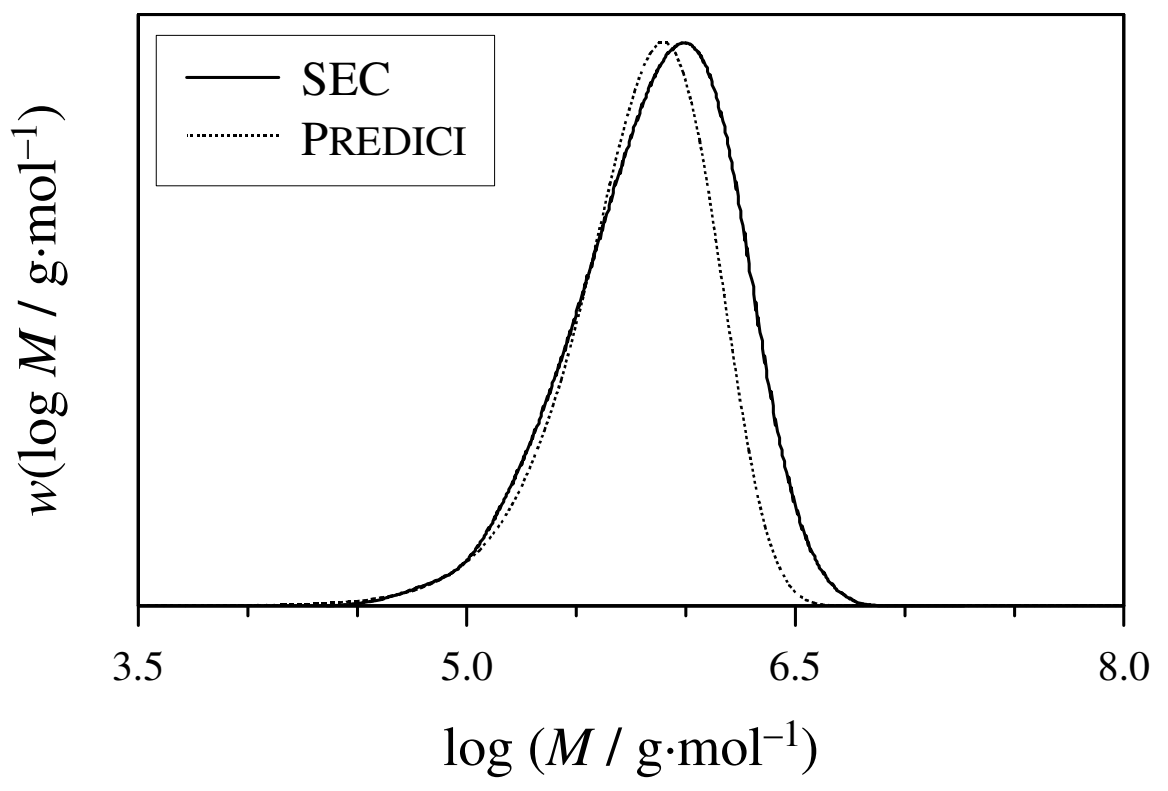

Fig. 9.35 Experimental molecular weight distribution (full line) of polyDMA (20 per cent of conversion) produced at $70{ }^{\circ} \mathrm{C}$ with $c_{\mathrm{TBPO}}=1.0 \cdot 10^{-1} \mathrm{~mol} \cdot \mathrm{L}^{-1}$ and its PREDICI® simulation (dotted line).

The measured MWD is broader than the simulated one. The reasons for it might be some additional reaction steps, such as chain transfer reactions, that were not taken into account here. These reactions may have an influence on the MWD making it broader. It should be pointed out that the ideal kinetic model despite some uncertainties in prediction of MWD describes conversion vs. time curves remarkably well.

\subsection{SUMMARY AND CONCLUSIONS}

From the steady-state free-radical polymerizations of BA, DA and DMA, the variation of the rate of polymerization and the coupled parameter $k_{\mathrm{p}} / k_{\mathrm{t}}^{0.5}$ have been determined as a function of temperature, monomer, and initiator concentrations. For BA and DA, an increase of $k_{\mathrm{p}} / k_{\mathrm{t}}^{0.5}$ has been obtained upon increasing monomer concentration and temperature. The same experiments for DMA did not show any significant change in $k_{\mathrm{p}} / k_{\mathrm{t}}^{0.5}$. The kinetic behavior observed for BA and DA has been assigned to the formation of mid-chain radicals. The presence of such radicals can also explain, that the reaction order with respect to monomer concentration is above unity. Using proposed analytical equations [15], $E_{\mathrm{A}}$ of the backbiting was estimated to be close to $50 \mathrm{~kJ} \cdot \mathrm{mol}^{-1}$.

The comparison of the values of termination rate coefficients for DMA obtained from different methods (Section 9.3.3) shows good agreement of the data. 


\subsection{REFERENCES}

[1] Scott, G. E.; Senogles, E. J. Macromol. Sci. Chem. 1970, A4, 1105.

[2] Scott, G. E.; Senogles, E. J. Macromol. Sci., Revs. Macromol. Chem. 1973, C9(1), 49.

[3] Scott, G. E.; Senogles, E. J. Macromol. Sci. Chem. 1974, A8, 753.

[4] Winderlich, W. Macromol. Chem. 1976, 177, 973.

[5] Kaszas, G.; Földes-Berezsnich, T.; Tüdos; F. Eur. Polym. J. 1983, 19, 469.

[6] Daimon, H.; Kumanoteni, J. Macromol. Chem. 1975, 176, 2359.

[7] Madruga, E.; Fernandez-Garcia, M. Macromol. Chem. Phys. 1996, 197, 3743.

[8] McKenna, T. F.; Villanueva, A.; Santos, A. J. Polym. Sci. Part A: Polym. Chem. 1999, 37,571 .

[9] Fernandez-Garcia, M.; Fernandez-Sant, M.; Madruga, E. Macromol. Chem. Phys. 2000, 201, 1840.

[10] Rätzsch, M.; Zschach, I. Plaste und Kautschuk 1968, 15, 792.

[11] Otsu, T.; Ito, T.; Imoto, M. J. Polymer Sci., Polymer Simposia 1967, 16,2121.

[12] Vana, P.; Davis, T. P.; Barner-Kowolik, C. Aust. J. Chem. 2002, 55, 315.

[13] Moad, G.; Solomon, D. H. "The Chemistry of Free Radical Polymerization", 1st edition, Elsevier Science Inc., New York 1995, 231.

[14] Arzamendi, G.; Plessis, C.; Leiza, J. R.; Asua, J. M. Macromol. Theory Simul. 2003, 12,315 .

[15] Nikitin, A. N.; Hutchinson, R. A. Macromolecules 2005, 38, 1584.

[16] Polymer Handbook, 3rd ed.; Brandup, A.; Immergut, E. H., Eds.; Wiley-Interscience: New York, 1989.

[17] Gladishev, G. P.; Rafikov, S. R. Russ. Chem. Revs. 1966, 35, 405.

[18] Kamachi, M.; Fujii, M.; Ninomiya, S.-I.; Katsuki, S.; Nozakura, S.-I. J. Polym. Sci. Polym. Chem. Ed. 1982, 20, 1489.

[19] Maxwell, I. A.; Napper, D. H.; Gilbert, R. G. J. Chem. Soc., Faraday Trans. 1 1987, $83,1449$.

[20] Horie, K.; Mita, I.; Kambe, H. J. Polym. Sci., Part A-1 1968, 6, 2663.

[21] Sandmann, J. Ph. D. Thesis, Göttingen, 2000.

[22] Müller, M. Ph. D. Thesis, Göttingen, 2005.

[23] Busch, M. Macromol. Theory Simul. 2001, 10, 262.

[24] Buback, M.; Egorov, M.; Junkers, T.; Panchenko, E. Macromol. Chem. Phys. 2005, $206,333$. 
[25] Beuermann, S.; Paquet Jr., D. A.; McMinn, J. H.; Hutchinson, R. A. Macromolecules 1996, 29, 4206.

[26] Lyons, R. A.; Hutovic, J.; Piton, M. C.; Christie, D. I.; Clay, P. A.; Manders, B. G.; Kable, S. H.; Gilbert, R. G. Macromolecules 1996, 29, 1918.

[27] Kowollik, C. Ph. D. Thesis, Göttingen, 1999.

[28] Beuermann, S.; Buback, M. Prog. Polym. Sci. 2002, 27, 191.

[29] Buback, M.; Kurz, C. H.; Schmaltz, C. Macromol. Chem. Phys. 1998, 199, 1721.

[30] Mahabadi, H. K.; O’Driscoll, K. F. Macromol. Chem. 1978, 179, 1921.

[31] Moritz, U.; Mayerhoff, G. Macromol. Chem. 1970, 139, 23.

[32] Lazar, M.; Hrckova, L.; Borsig, E. J. Macromol. Sci.-Pure Appl. Chem. 2002, A39, 365 .

[33] Malavasic, T.; Osredkar, U.; Anzur, I.; Vizovisek, I. J. Mac. Sci., Chem. 1986, A23, 853.

[34] Beuermann, S. Ph. D. Thesis, Göttingen, 1993.

[35] Tulig, T. J.; Tirrell, M. Macromolecules 1982, 15, 459.

[36] Garcia, N. Unpublished results.

[37] Buback, M.; Egorov, M.; Gilbert, R. G.; Kaminsky, O. F.; Olaj, G. T.; Russell, G. T.; Vana, P.; Zifferer, G. Macromol. Chem. Phys. 2002, 203, 2570. 


\section{APPENDIX}

\section{Abbreviations}

$a$

$\alpha$

A

A

B

$c_{\mathrm{i}}$

$c_{\mathrm{I}}$

$c_{\mathrm{M}}$

$c_{\mathrm{M}}{ }^{0}$

$c_{\mathrm{R}}$

$c_{\mathrm{R}}{ }^{0}$

$c_{\mathrm{R}, \mathrm{I}}$

$c_{\mathrm{X}}$

$C_{\text {tr }, \mathrm{X}}$

$E_{\mathrm{A}}$

$E_{\lambda}$

$E_{\mathrm{p}}$

$\varepsilon$

$f$

$f_{\mathrm{i}}$

$f^{0}{ }$ i

$F_{\mathrm{i}}$

$\Phi$

[ $\eta]$

HV

$I^{0}$
Mark-Houwink parameter

exponent of the chain-length dependence of $k_{\mathrm{t}}$

pre-exponential factor

absorbance

integrated molar absorbtivity

concentration of substance $\mathrm{i}$

initiator concentration

(overall) monomer concentration

initial monomer concentration

overall radical concentration

effective initial radical concentration

effective initiator radical concentration

concentration of chain transfer agent

rate constant of chain transfer to $\mathrm{X}$

activation energy

energy of one mole of photons at the wave

length $\lambda$

energy of one laser pulse

molar absorption coefficient

initiator efficiency

mole fraction of component $i$ in the comonomer mixture

initial mole fraction of component $i$ in the

comonomer mixture

mole fraction of component $i$ in a binary

copolymer

primary quantum yield

intrinsic viscosity

hydrodynamic volume

intensity of light going to the sample cell 
I

$i$

$i_{\mathrm{c}}$

K

$k_{\mathrm{bb}}$

$k_{\mathrm{d}}$

$k_{\mathrm{p}}$

$k_{\mathrm{p}, \mathrm{ac}}$

$k_{\mathrm{pii}}$

$k_{\mathrm{pij}}$

$k_{\mathrm{pijk}}$

$k_{\mathrm{p}, \text { copo }}$

$k_{\mathrm{p}, \mathrm{mac}}$

$k_{\mathrm{t}}$

$k_{\mathrm{t}, \mathrm{c}}$

$k_{\mathrm{t}, \mathrm{d}}$

$<k_{\uparrow}>$

$k_{\mathrm{t}}(i, j)$

$k_{\mathrm{t}}^{0}$

$k_{\mathrm{tr}, \mathrm{X}}$ intensity of light coming from the sample cell chain length size at which a radical might start displaying long-chain behavior with regard to termination Mark-Houwink parameter rate coefficient of backbiting rate coefficient of initiator decomposition propagation rate coefficient of a homopolymerization propagation rate coefficient of an acrylate homopolymerization propagation rate coefficient in a homopolymerization of monomer $i$ propagation rate coefficient for the reaction of a macroradical with terminal unit $i$ and monomer $j$ propagation rate coefficient for the reaction of a macroradical with penultimate unit $i$ and terminal unit $\mathrm{j}$ with a monomer $\mathrm{k}$ (average) propagation rate coefficient in a binary copolymerization propagation rate coefficient of a methacrylate homopolymerization

termination rate coefficient termination rate coefficient for termination by combination termination rate coefficient for termination by disproportionation

chain-length averaged termination rate coefficient termination rate coefficient between radicals of chain length $i$ and $j$

termination rate coefficient between two monomer radicals rate coefficient of chain transfer to $\mathrm{X}$ 


\begin{tabular}{|c|c|}
\hline$L$ & degree of polymerization \\
\hline$l$ & optical path length \\
\hline$M$ & molar mass \\
\hline $\mathrm{M}_{\mathrm{i}}$ & monomer of type i \\
\hline$n_{\mathrm{abs}}$ & number of absorbed photons \\
\hline$N_{\mathrm{av}}$ & Avogadro constant \\
\hline$\nu$ & pulsed laser frequency \\
\hline$\tilde{v}$ & specific wave number \\
\hline$\nu_{\text {in }}$ & $\begin{array}{l}\text { effective rate of initiation in chemically initiated } \\
\text { polymerization }\end{array}$ \\
\hline$p$ & pressure \\
\hline$P_{n}$ & polymer (chain length $\mathrm{n}$ ) \\
\hline $\mathrm{R}_{\mathrm{i}}$ & macroradical with terminal unit $\mathrm{i}$ \\
\hline$R_{i j}$ & $\begin{array}{l}\text { macroradical with penultimate unit } \mathrm{i} \text { and terminal } \\
\text { unit } \mathrm{j}\end{array}$ \\
\hline$R_{\mathrm{p}}$ & overall polymerization rate \\
\hline$r_{\mathrm{i}}$ & $\begin{array}{l}\text { reactivity ratio (homo propagation to cross } \\
\text { propagation) }\end{array}$ \\
\hline$\rho$ & density \\
\hline$s_{\mathrm{i}}$ & radical reactivity ratio \\
\hline$t$ & time \\
\hline$t_{0}$ & time between two laser pulses \\
\hline$T$ & absolute temperature \\
\hline$\Theta$ & temperature in ${ }^{\circ} \mathrm{C}$ \\
\hline$\Delta V^{ \pm}$ & activation volume \\
\hline$V$ & volume \\
\hline $\mathrm{X}$ & transfer agent \\
\hline$X$ & conversion \\
\hline
\end{tabular}




\section{DANKSAGUNG}

Mein besonderer Dank gilt Herrn Professor Dr. M. Buback für die interessante Themenstellung sowie die stete Förderung und Unterstützung dieser Arbeit.

Der Deutschen Forschungsgemeinschaft danke ich für die finanzielle Unterstützung im Rahmen des Graduiertenkollegs “Microstructural Control in Free-Radical Polymerization”.

Herrn Dr. H. P. Vögele danke ich für die Anregungen und Tipps bei der Bewältigung apparativer und technischer Probleme.

Ein besonderer Dank gilt auch Thomas Junkers und Mark Egorov für die gute Zusammenarbeit, die vielen anregenden Diskussionen und die gemeinsame Arbeit an neuen Ideen.

Heike Rohmann danke ich für die Reinigung der Monomere und den Spaß während der Arbeit.

Thomas Junkers und Moritz Gadermann danke ich herzlich für das aufmerksame Korrekturlesen dieser Arbeit.

Allen Mitgliedern der Abteilung möchte ich für ein freundliches Arbeitsklima, sowie stete Hilfs- und Diskussionsbereitschaft danken. Unsere Ausflüge (wie Boßeln und den Ausflug zur Plesse mit dem Fahrrad im Regen) werde ich nie vergessen. Spezieller Dank gilt meinen Bürokollegen Dorit Nelke, Matthias Müller, Björn Steisel und Toshihiko Arita sowie Stefan Hinrichs und Nuria Garcia für die nette Arbeitsatmosphäre.

Besonders herzlich möchte ich mich bei meinen Eltern bedanken, die mir mein Studium ermöglicht und mich jederzeit unterstützt haben.

Schließlich möchte ich mich bei meinem Mann Matthias bedanken. Ohne Dich wäre alles nicht so schön gewesen. 


\section{Curriculum Vitae}

\section{Personal information}

Name

22.05.1979

22.07.2004

\section{Education}

Sept 1986 - Mai 1989

Sept 1989 - Mai 1996

Sept 1996 - June 2001

Sept 1999 - June 2001

Since Sept 2001

Sept 2001 - Sept 2004
Elena Müller (born Panchenko)

Born in Jarowoje, Altai region, Russia

Marriage to Matthias Müller

Elementary school in Jarowoje, Russia

Secondary school in Jarowoje, Russia

Novosibirsk State University, Department of Natural Sciences, Novosibirsk, Russia

Graduation at Novosibirsk State University in the Institute of Catalysis in the research group of Prof. Dr. V. A. Kirillov. Theme: "Investigation of Copper Catalysts distributed on a Heat-Exchange Surface in the Hydrogenation of Nitrobenzene to Aniline"

$\mathrm{Ph}$. D. at the University of Göttingen in the Institute of Physical Chemistry in the research group of Prof. Dr. M. Buback. Theme: "Detailed Investigations into the Propagation and Termination Kinetics of Bulk Homo- and Copolymerization of (Meth)Acrylates"

Member of the European Graduate School (EGS) "Microstructural Control in Free-Radical Polymerization"

\section{Experience}

Apr 2003 - March 2005

Since Sept 2001
Student assistant at the University of Göttingen Scientific worker at the University of Göttingen 